Pontifícia Universidade Católica

DO RIO DE JANEIRO

Fábio Jessen Werneck de Almeida Martins

Characterization of Near-Wall Turbulent

Flows by Tomographic PIV

Tese de Doutorado

Thesis presented to the Programa de Pós-Graduação em Engenharia Mecânica of the Departamento de Engenharia Mecânica, PUC-Rio, as partial fulfillment of the requirements for the degree of Doutor em Engenharia Mecânica.

Advisor: $\quad$ Prof. Luis Fernando Alzuguir Azevedo

Co-Advisor:

Prof. Jean-Marc Foucaut

Co-Advisor:

Prof. Michel Stanislas

Rio de Janeiro

September 2016 
Pontifícia Universidade Católica

DO RIO DE JANEIRO

Fábio Jessen Werneck de Almeida Martins

\title{
Characterization of Near-Wall Turbulent Flows by Tomographic PIV
}

Thesis presented to the Programa de Pós-Graduação em Engenharia Mecânica of the Departamento de Engenharia Mecânica do Centro Técnico Científico da PUC-Rio, as partial fulfillment of the requirements for the degree of Doutor.

\author{
Prof. Luis Fernando Alzuguir Azevedo \\ Advisor \\ Departamento de Engenharia Mecânica - PUC-Rio \\ Prof. Jean-Marc Foucaut \\ Co-Advisor \\ École Centrale de Lille, France \\ Prof. Michel Stanislas \\ Co-Advisor \\ École Centrale de Lille, France \\ Prof. Fulvio Scarano \\ Delft University of Technology, Netherlands
}

Prof. Julio Soria

Monash University, Australia

Prof. Roney L. Thompson

Federal University of Rio de Janeiro

Prof. Jean-Christophe Robinet

École Nationale Supérieure des Arts et Métiers, France

Prof. Márcio da Silveira Carvalho

Coordinator of the Centro Técnico Científico - PUC-Rio

Rio de Janeiro, September 26 ${ }^{\text {th }}, 2016$. 
All rights reserved.

Fábio Jessen Werneck de Almeida Martins

The author graduated as mechanical engineer and control and automation engineer in 2007 from PUC-Rio, and got his Master's degree as electrical engineer in 2010 from the same institution. This Doctoral Thesis was a joint work between PUC-Rio and École Centrale de Lille in a double-diploma program.

Bibliographic data

Martins, Fábio Jessen Werneck de Almeida

Characterization of Near-Wall Turbulent Flows by Tomographic PIV / Fábio Jessen Werneck de Almeida Martins; advisor: Luis Fernando Alzuguir Azevedo; coadvisors: Jean-Marc Foucaut, Michel Stanislas - 2016.

251 f: il. color ; $30 \mathrm{~cm}$

1. Tese (doutorado) - Pontifícia Universidade Católica do Rio de Janeiro, Engenharia Mecânica, 2016.

Inclui bibliografia.

1. Engenharia Mecânica - Teses. 2. BIMART. 3. Turbulência. 4. Escoamento em Canal. 5. Camada Limite. I. Azevedo, Luis Fernando Alzuguir. II. Foucaut, JeanMarc. III. Stanislas, Michel. IV. Pontifícia Universidade Católica do Rio de Janeiro. Departamento de Engenharia Mecânica. V. Título. 
This work is dedicated to my mother Magali, my father Manuel and my brother Rafael. 


\section{Acknowledgments}

This thesis would not have been realized without the help of many people who supported and motivated me during these years.

I would like to thank my supervisors, Professors Luis Fernando Azevedo, Michel Stanislas and Jean-Marc Foucaut, for their guidance and support throughout the periods spent both in Brazil and France.

I am grateful to Prof. Fulvio Scarano, Prof. Julio Soria, Prof. Roney L. Thompson and Prof. Jean-Christophe Robinet for accepting to be jury members.

I am thankful to my colleagues at PUC-Rio and at École Centrale de Lille who made me loved these places.

Thanks to the staff at PUC-Rio and at École Centrale de Lille who was so efficient and helpful throughout the entire process.

I would like to acknowledge all the team that worked on the LML boundarylayer tomo-PIV experiment.

I am grateful to Dr. Lionel Thomas for his work on the tomo-PIV software. The present thesis would not be possible without his help.

I would like to thank Dr. Judith Vatteville for her collaboration on tomo-PIV comparison, processing samples of the boundary-layer data by LaVision DaVis software.

This work was funded by the Mechanical Engineering Department of PUC-Rio and Brazilian scholarships CAPES (grant number BEX 9249/12-5) and, after, CNPq (grant number 161857/2015-9). The experiment carried out at LML had a financial support of AFDAR European project, ANR Vive3D contract and CISIT.

I would like to thank my friends for their conversations, dinners, beers and everything else.

A special thanks goes to my girlfriend for her patience, encouragement and, of course, for happy moments at her side.

I do not have words to express my feelings for my family. Their love, support and understanding have inspired me throughout my life. 


\section{Abstract}

Martins, Fábio Jessen Werneck de Almeida; Azevedo, Luis Fernando Alzuguir (advisor); Foucaut, Jean-Marc (co-advisor); Stanislas, Michel (co-advisor). Characterization of Near-Wall Turbulent Flows by Tomographic PIV. Rio de Janeiro, 2016. 251p. Doctoral Thesis - Departamento de Engenharia Mecânica, Pontifícia Universidade Católica do Rio de Janeiro.

The fundamental study of turbulent structures near a wall is of significant importance due to its dominant role in innumerous engineering applications. In turbulent flows, eddies of different scales interact with each other in a complex phenomenon of a continuous self-sustaining process. Although the presence of patterns of coherent motion seem to be responsible for the maintenance of turbulence, the evolution and the interaction of these structures are still not completely understood. To investigate such phenomena, near-wall turbulent flows over a smooth flat plate in a wind tunnel and inside a square water channel were conducted. The present work employed a high-repetition tomographic PIV technique to measure three dimensional, time-resolved velocity fields. The tomo-PIV processing was optimized in order to improve the algorithm performance and the vector fields accuracy. Conditional statistics of coherent structures, namely, lowand high-speed regions, sweeps, ejections and vortices, were computed. Quantitative results showed vortical structures spread all over the flow, nevertheless, concentrated close to low-speed regions and ejections. Results indicated that vortical structures were indirectly correlated with the process of turbulence production, although they were the main responsible for the turbulent energy dissipation.

\section{Keywords}

BIMART; Turbulence; Channel Flow; Boundary Layer. 


\section{Resumo}

Martins, Fábio Jessen Werneck de Almeida; Azevedo, Luis Fernando Alzuguir (orientador); Foucaut, Jean-Marc (coorientador); Stanislas, Michel (co-orientador). Caracterização de Escoamentos Turbulentos Próximos à Parede Através de PIV Tomográfico. Rio de Janeiro, 2016. 251p. Tese de Doutorado - Departamento de Engenharia Mecânica, Pontifícia Universidade Católica do Rio de Janeiro.

O estudo fundamental de estruturas turbulentas próximas a paredes é de significativa importância devido ao seu papel dominante em inúmeras aplicações de engenharia. Em escoamentos turbulentos, estruturas de diferentes escalas interagem entre si através de um fenômeno complexo caracterizado por um processo contínuo e auto-sustentável. Embora a presença de padrões de movimento coerente pareça ser responsável pela manutenção da turbulência, a evolução e a interação destas estruturas ainda não são completamente entendidas. Para investigar tais fenômenos, foram realizados estudos de escoamentos turbulentos próximos a paredes sobre uma placa plana em um túnel de vento e dentro de um canal de água quadrado. O presente trabalho empregou uma técnica tomográfica de velocimetria por imagem de partícula de alta taxa de medição de campos de velocidade tridimensionais. O processamento da técnica tomo-PIV foi otimizado a fim de melhorar o desempenho do algoritmo e a exatidão dos campos vetoriais medidos. As medições realizadas permitiram a obtenção de estatísticas condicionais das estruturas coerentes típicas da camada limite turbulenta, tais como regiões de baixa e de alta velocidades, varreduras ("sweeps"), ejeções ("ejections") e vórtices. Os resultados quantitativos mostraram vórtices distribuídos por todo o escoamento, entretanto, concentrados na vizinhança das regiões de baixa velocidade e ejeções. Os resultados indicaram que os vórtices estavam indiretamente correlacionados com o processo de produção de turbulência, apesar de serem os principais responsáveis pela dissipação da energia turbulenta.

\section{Palavras-chave}

BIMART; Turbulência; Escoamento em Canal; Camada Limite. 


\section{Résumé}

Martins, Fábio Jessen Werneck de Almeida; Azevedo, Luis Fernando Alzuguir (directeur de thèse); Foucaut, Jean-Marc (codirecteur de thèse); Stanislas, Michel (codirecteur de thèse). Caractérisation des Écoulements Turbulents à Proximité d'une Paroi par PIV Tomographique. Rio de Janeiro, 2016. 251p. Thèse de Doctorat - Departamento de Engenharia Mecânica, Pontifícia Universidade Católica do Rio de Janeiro.

L'étude fondamentale des structures turbulentes proche d'une paroi est d'une significative importance en raison de son rôle dominant dans nombreuses applications d'ingénierie. Dans les écoulements turbulents, des structures d'échelles différentes interagissent dans un phénomène complexe caractérisé par un processus autonome et continu. Bien que la présence de modèles de mouvements cohérents semble entretenir la turbulence, l'évolution et l'interaction de ces structures ne sont pas encore complètement compris. Pour analyser ces phénomènes, l'étude d'écoulements turbulents à proximité de paróis a été effectuées sur une plaque plane dans une soufflerie et à l'intérieur d'un canal hydraulique de section carrée. Une technique tomographique de vélocimétrie par images de particules pour mesurer de champs de vitesse tridimensionnel a été employée. Le traitement des données a été optimisé afin d'améliorer la précision des champs de vecteurs mesurés. Les mesures realisés ont permis d'obtenir des statistiques conditionnelles des structures cohérentes, à savoir, les régions à faible et à grande vitesse, les "sweeps", les "ejections" et les tourbillons. Les résultats quantitatives ont montré des structures tourbillonnaires distribués sur tout le flux, cependant, concentrée au voisinage des régions à faible vitesse et des "ejections". Les résultats ont indiqué que les structures tourbillonnaires étaient indirectement corrélés avec le processus de production de la turbulence, bien qu'ils ont été les principaux responsables de la dissipation d'énergie turbulente.

\section{Mots-clefs}

BIMART; Turbulence; Débit en Canal; Couche Limite. 


\section{Contents}

1 Introduction $\quad 28$

1.1 Objective 31

2 Wall Turbulence $\quad 33$

2.1 Brief Introduction to Turbulence 33

2.2 Boundary Layer Flow 36

$\begin{array}{lll}2.3 & \text { Channel Flow } & 38\end{array}$

2.4 Turbulent Structures $\quad 41$

Low- and High-Speed Regions $\quad 41$

Ejections and Sweeps 42

Vortices $\quad 43$

Turbulent Structure Organization and Interaction 46

3 Tomographic PIV $\quad 50$

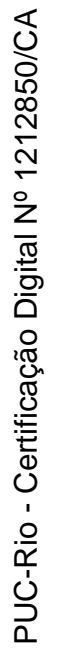

3.1 Tomographic PIV Developments 50

3.2 Working Principle $\quad 55$

Particle Imaging and Concentration $\quad 56$

Volume Illumination $\quad 58$

Camera Arrangement $\quad 59$

System Calibration $\quad 60$

Reconstruction Techniques 63

$3 D$ Motion Analysis $\quad 66$

$\begin{array}{lll}3.3 & \text { Quality Indicators } & 67\end{array}$

Standard Reconstruction Quality 68

Re-Projection Quality $\quad 69$

Particle Distribution Signal-to-Noise Ratio $\quad 69$

Light Intensity Signal-to-Noise Ratio 70

Normalized Intensity Variance 71

Reconstructed Particle Characteristics 71

Error in Particle Position 73

Error in Velocity Measurements 73

Uncertainty in Velocity Measurements 73

Experimental Optimization Using Quality Indicators $\quad 74$

4 Boundary Layer Experiment $\quad \mathbf{7 6}$

$\begin{array}{lll}4.1 & \text { Wind Tunnel } & 76\end{array}$

$\begin{array}{lll}4.2 & \text { Tomo-PIV Setup } & 77\end{array}$

$\begin{array}{lll}4.3 \text { Calibration } & 81\end{array}$

5 Tomo-PIV Optimization Results $\quad 94$

5.1 Volume Reconstruction Optimization 94

Image Pre-Processing $\quad 95$ 
Volume Filtering $\quad 98$

Number of Cameras $\quad 100$

Particle Density 101

Summary of Tomo-PIV Optimization 102

5.2 Comparison of Optimized Reconstruction Algorithms 103

Convergence of Reconstruction Algorithms 103

Reconstruction Algorithms 105

$\begin{array}{ll}\text { Velocity Statistics } & 107\end{array}$

Comparison With Other Tomo-PIV Softwares 110

Summary of the Comparison of Algorithms 116

6 Boundary Layer Results $\quad 117$

$\begin{array}{lll}6.1 & \text { Overall Flow Statistics } & 117\end{array}$

Summary of Overall Flow Statistics 129

6.2 Flow Structure Statistics 131

Pattern Recognition 131

Low- and High-Speed Regions 137

Ejections and Sweeps 152

Vortices 160

Summary of Flow Structure Statistics 184

7 Square Channel Experiments $\quad 189$

7.1 Square Water Channel 189

7.2 Tomo-PIV Setup 193

7.3 Volume Illumination 194

$\begin{array}{lll}7.4 & \text { Seeding } & 197\end{array}$

7.5 Experimental Procedure 200

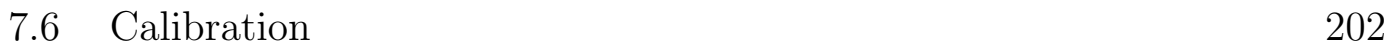

8 Square Channel Results 209

8.1 Tomo-PIV Processing 209

8.2 Flow Analysis 213

8.3 Summary of the Water Channel 223

9 Conclusions and Remarks $\quad 225$

10 Bibliography 233 


\section{List of Figures}

1.1 Movements in the study of turbulence ([McDonough, 2007]).

1.2 (a) Sketch of the turbulent water motion by Leonardo da Vinci (Codex Atlanticus), showing the unsteady eddying motions. (b) Middle-cut volume through a transitional jet calculated by PTV-STB and visualized by FlowFit (adapted from [Schanz et al., 2016]). Instantaneous isosurfaces of vorticity with superimposed particle tracks, extending 5 time-steps back (left). Isosurfaces of streamwise acceleration at the same time-step (right).

2.1 Structure of the flat plate turbulent boundary layer as visualized with the hydrogen bubble technique of [Kline et al., 1967]. The wire is located parallel to the plate and normal to the direction at of flow at $y^{+}=9$. The flow is from top to bottom of the picture. The bubbles were collected into long streaks.

2.2 (a) Isometric schematic view of single hairpin vortex and (b) dual-view picture of dye-market single hairpin vortex from the turbulent boundary-layer experiments of [Smith et al., 1991] 44

2.3 Coherent vortices extracted from the direct numerical simulation of a flow inside a duct with $R e_{\tau}=180$ ([Vinuesa et al., 2014]). Colour code by wall-normal direction: blue refers to vortices closer to the lower wall, while red to the ones closer to the upper wall. Flow is from lower-left corner to upper-right. $h$ is the half channel height.

2.4 Illustration of horseshoe vortex ([Theodorsen, 1952]).

2.6 Organization model of [Adrian et al., 2000b] in near wall turbulence, $U_{c 1}, U_{c 2}$ and $U_{c 3}$ are the convection velocities of each hairpin packet respectively.

2.7 Instantaneous vortex distribution detected by the $\mathrm{Q}$ criterion (green) and low-speed zones of $u<0.89 U$ (blue) for $0.34<$ $y / \delta<0.67$ ([Elsinga et al., 2010]).

3.1 Formation of ghost particles in a 2-camera tomo-PIV setup. ([Elsinga, 2008]).

3.2 Instantaneous water-tunnel tomo-PIV result at $R e=8000$ ([Schanz et al., 2013b]). Isosurfaces of 3D swirl strength $\lambda_{c i}$ with vector slices color-coded by streamwise velocity $u$.

3.3 Working principle of tomographic PIV (adapted from [Elsinga et al., 2006]).

3.4 Light scattered by (a) $1 \mu \mathrm{m}$ oil particle in air and (b) $10 \mu \mathrm{m}$ glass particle in water employing a wavelength $\lambda$ of $532 \mathrm{~nm}$ (adapted from [Raffel et al., 2007]). The polar distribution of the scattered light intensity is in logarithmic scale. 
3.5 Reconstruction errors due to tomographic angular aperture for a 2-camera system adapted from [Scarano, 2013]. The sketch (a) refers to an angular aperture of $60^{\circ}$ and (b) to an aperture of $40^{\circ}$. The line-of-sights are displayed as dash-dot lines, the actual particles as orange circles and reconstructed particles as blue regions.

3.6 Sketch of some camera configurations studied by [Thomas et al., 2014].

3.7 (a) Residual triangulation disparities $\boldsymbol{d}_{i}(X, Y, Z)=\left(x_{i}, y_{i}\right)-$ $\left(x_{i}^{\prime}, y_{i}^{\prime}\right)$ due to calibration errors. (b) Sample of a disparity map of one of the cameras for a single sub-volume. (Adapted from [Wieneke, 2008].)

3.8 Volume reconstruction from two-camera tomographic PIV reproduced from [Scarano, 2013].

3.9 Sample of normalized intensity profile of reconstructed light by MART from the boundary-layer experiment.

4.1 LML wind tunnel.

4.2 Blades fixed in a translation stage to compose the knife-edge filter.

4.3 Sketch of the boundary-layer experimental setup.

4.4 Pictures of the (a) side and (b) front views of the boundarylayer experimental setup.

4.5 (a) Detail of the optical path of the laser light before the knife-edge filter. The light reflected in guiding mirrors, pass trough a diverging lens and a cylindrical lens. (b) Laser light sheet coming from the top of the wind tunnel. The system synchronizer, step-motor control of the calibration target and the multimeter are also visible in this picture.

4.6 Samples of particle image concentration of (a) 0.010, (b) 0.029, (c) 0.057 and (d) $0.070 \mathrm{ppp}$ recorded by camera 1 . The brightness and contrast of the images were adjusted to enhance visualization.

4.7 Calibration target fixed at the translation unit in order to record multiple planes. Detail of (a) the target plane at the position $z^{*}=0$ at the middle of the laser volume and (b) the light source arrangement during the calibration recordings.

4.8 Target image for each camera. The images show the projection of the reconstructed volume limits, the image coordinate system (in red), the world coordinate system (in yellow) and the wind-tunnel coordinate system (in white). In order to enhance the visualization the third axis from the world and wind-tunnel systems can be suppressed sometimes.

4.9 Refraction phenomena in the target glass showing (a) the distance (in $\mathrm{mm}$ ) between the real target marker (black point) to the virtual ones (red points) and (b) the virtual planes adopted (dashed red lines) instead of the real target plane (dash-dotted grey line). 
$4.102 \mathrm{D}$ disparity map of camera 1 at the middle of the laser sheet for the translated-plane self-calibration method with a search region of 3 pixels.

4.11 Amplified views of the upper-left corner of disparity maps for search region of 3 pixels: standard method, 4-camera limited algorithm and translated-plane self-calibration procedure.

4.12 Amplified views of the upper-left corner of disparity maps for search region of 3 pixels after the translated-plane selfcalibration correction.

4.13 Contour of the base of investigation volume projected onto the camera images of light reflections. The brightness and contrast of the time-history minimum intensity images were adjusted to enhance visualization.

5.1 Sample of the original image from camera 1 (a) and after the background subtraction by subMin (b), subMedian (c) and subMean (d) filters. The images were normalized to enhance the visualization.

5.2 Influence of different background-subtraction filters combined with low-pass Gaussian filter on the intensity profile of reconstructed light for a 5-iteration standard MART.

5.3 Influence of volume threshold filter (vt) and volume Gaussian filter (vgf) (a) on the intensity profile of reconstructed light, (b) on the probability density function of the normalized peak intensity, (c) on the particle concentration per volume and (d) on the particle size for a 5-iteration standard MART.

5.4 Influence of (a) the number of cameras and (b) the particle density on the intensity profile of the reconstructed light for a 5-iteration standard MART.

5.5 Convergence of mean re-projection quality for MART-standard, MART-bilinear, BIMART and SMART algorithms.

5.6 Convergence of $\left\langle\boldsymbol{E}_{\text {in }}>\right.$ (signal) and $<\boldsymbol{E}_{\text {out }}>$ (noise) for MART-standard, MART-bilinear, BIMART and SMART algorithms.

5.7 Convergence of reconstructed light profiles for (a) MART-standard, (b) MART-bilinear, (c) BIMART and (d) SMART algorithms.

5.8 Comparison of (a) the averaged intensity profile, (b) the computational time required for one reconstruction, (c) the averaged particle size and (d) particle concentration for MART-standard, MART-bilinear, BIMART and SMART algorithms.

5.9 Comparison of instantaneous velocity field reconstructed by different methods: (a) MART, (b) BIMART and (c) SMART. The colour represents the third component and the black isolines the Q-criterion $=1500 \mathrm{~s}^{-2}$. 
5.10 Plots of (a) velocity profile and (b) turbulence fluctuations normalized with respect of the viscous inner scale for MART-standard, BIMART and SMART algorithms.

5.11 Probability density functions at $y^{+}=50$ for (a) $u^{\prime} / \sqrt{\left\langle u^{\prime 2}\right\rangle}$, (b) $v^{\prime} / \sqrt{<v^{\prime 2}>}$ and (c) $w^{\prime} / \sqrt{<w^{\prime 2}>}$.

5.12 Comparison between reconstructed particles in the center region of the reconstructed volume computed by (a) the code of the present work and (b) Davis software.

5.13 Comparison between instantaneous velocity fields computed by (a) the software of the present work and (b) Davis software. The colour represents the third velocity component. From top to bottom, each row of figures represents, respectively, time instants $0.005 \mathrm{~s}, 0.605 \mathrm{~s}$ and $1.205 \mathrm{~s}$.

6.1 Sample of 3D tomo-PIV velocity field in the boundary-layer experiment. The color code refers to the streamwise velocity. One over three $2 \mathrm{D}$ vectors of wall-normal and spanwise velocity components are also plotted.

6.2 Six consecutive instantaneous velocity fields on the middle plane of tomo-PIV measurements of the boundary-layer experiment. Colour code related to the instantaneous stream-wise velocity and vectors related to the wall-normal and spanwise instantaneous velocity components.

6.3 Interaction among low-speed streaks (green isosurfaces of $u=1.4 \mathrm{~m} / \mathrm{s}$ ) and turbulent vortical structures (blue isosurfaces of Q-criterion $=8000 \mathrm{~s}^{-1}$ ) inside a turbulent boundary layer from (a) raw and (b) filtered data.

6.4 (a) Mean streamwise velocity in the middle of the volume $(x=0)$ and (b) profile of streamwise velocity.

6.5 Profiles of divergence and uncertainty in velocity field in relation to the distance to the wall for the tomo-PIV boundarylayer experiment.

6.6 Profiles of mean streamwise velocity in (a) semi-log and (b) linear plots.

6.7 Profiles of turbulent velocity fluctuations made dimensionless by (a) wall-units and by (b) the mean kinetic energy. Comparison with hot-wire anemometer measurements (HWA) and DNS of [Hoyas and Jiménez, 2008].

6.8 Profiles of (a) Reynolds stress and (b) viscous stress. Comparison with hot-wire anemometer measurements (HWA), Van Driest profile and DNS of [Hoyas and Jiménez, 2008].

6.9 Profiles of dissipation for tomo-PIV, hot-wire anemometer measurements (HWA) and DNS of [Hoyas and Jiménez, 2008] in relation to the distance to the wall.

6.10 Profiles of root-mean-square of fluctuating vorticity components. Comparison of tomo-PIV boundary-layer data with DNS of [Hoyas and Jiménez, 2008]. One over two points are plotted for clarity. 
6.11 Kolmogorov length scale in the tomo-PIV boundary-layer experiment plotted with respect to the wall distance.

6.12 (a) Autocorrelation coefficient of streamwise velocity for positive spatial lags (continuous blue line) together with its osculating parabola (red dashed line) showing the Taylor micro-scale at $y+=100$. (b) Taylor micro-scale computed by the autocorrelation of streamwise velocity for different wall-normal positions for tomo-PIV and HWA techniques.

6.13 Taylor length scales computed by the local isotropic turbulence hypothesis with respect to the distance to the wall from tomo-PIV experimental data.

6.14 Probability density functions at $y^{+}=50$ for (a) $u^{\prime} / \sqrt{\left\langle u^{\prime 2}\right\rangle}$, (b) $v^{\prime} / \sqrt{<v^{\prime 2}>}$ and (c) $w^{\prime} / \sqrt{<w^{\prime 2}>}$.

6.15 Power spectral density for the tomo-PIV streamwise fluctuation velocity component at 100 wall units as a function of (a) frequency and (b) the inverse of Kolmogorov length scale.

6.16 Dilation operation of a square object with a hole in the middle by a diamond structuring element. The result is a larger object without the hole.

6.17 Erosion operation of two objects by a diamond structuring element. The result is a smaller bottom-left object and the suppression of the upper-right small object.

6.18 Watershed segmentation proceeds by computationally immersing the underside region as a catchment basin (from [Rettmann et al., 2000]).

6.19 Watershed transform by immersion on the 4-connected grid, showing relabelling of watershed positions. (a) Original image; (b-e) labelling steps based on eq. (6.2.7) (from [Roerdink and Meijster, 2000]).

6.20 PDF of (a) $u^{\prime}$ and (b) $u^{\prime} / u_{\mathrm{rms}}^{\prime}$ values used to detect low- and high-speed regions for different wall-normal distances.

Low- and high-speed region detection procedure in the the middle plane of the tomo-PIV data: (a) colorplot of $u^{\prime} / u_{\text {rms }}^{\prime}$, (b) binary field of low-speed regions after thresholding, (c) lowspeed regions after closing, opening and cleaning operations and (d) binary field of high-speed regions after same binary operations.

6.22 Filtered binary fields of low-speed regions in the the middle plane of the tomo-PIV data for different threshold levels: (a) 1, (b) -1.5, (c) -2 and (d) -2.5 .

6.23 Frequency of occurrence of the centre of turbulent structure detected by standard and normalized fluctuating streamwise velocity for different wall-normal distances. (a) Low- and (b) high-speed regions.

6.24 Projection angles based on the fluctuating velocity vector and definition of quadrants respectively on streamwise-wallnormal, streamwise-spanwise and wall-normal-spanwise planes. 147 
6.25 PDF of fluctuating streamwise, wall-normal and spanwise velocities of (a) low- and (b) high-speed regions for different wall-normal positions.

6.26 Mean fluctuating velocity components of (a) low- and (b) highspeed regions for different wall-normal positions.

6.27 Root-mean-square of fluctuating velocity components of (a) low- and (b) high-speed regions for different wall-normal positions. Flow statistics are also plotted for comparison.

6.28 (a) Reynold stresses and (b) dissipation rate of low- and high-speed regions in relation to wall-normal distance. Flow statistics are also presented for comparison.

6.29 PDF of fluctuating streamwise, wall-normal and spanwise vorticities and magnitude of the vorticity vector at (a) lowand (b) high-speed structures for different wall-normal positions. 150

6.30 Root-mean-square of streamwise, wall-normal and spanwise vorticities computed in high-speed regions as a function of the distance to the wall. Flow statistics are also plotted for comparison.

6.31 Probability density function of projection angles onto the streamwise-wall-normal plane, streamwise-spanwise plane and wall-normal-spanwise plane of (a) low- and (b) high-speed regions for different wall-normal positions.

6.32 Ejection-sweep definitions for momentum transport as proposed by [Wallace et al., 1972]. Events in quadrants 2 and 4 define sweeps and ejections, respectively.

6.33 PDF of (a) $u^{\prime} v^{\prime}$ and (b) $u^{\prime} v^{\prime} /<u^{\prime} v^{\prime}>$ values used to detect ejection and sweep events for different wall-normal distances.

6.34 Ejection and sweep detection procedure in the the middle plane of the tomo-PIV data: (a) colorplot of $F_{d}^{a}$, (b) binary field of ejection regions after thresholding, (c) ejection regions after closing, opening and cleaning operations and (d) binary field of sweep regions after same binary operations.

6.35 Frequency of occurrence of the centre of turbulent structure detected by standard and normalized Reynolds stress for different wall-normal distances. (a) Ejection and (b) sweep regions.

PDF of fluctuating streamwise, wall-normal and spanwise velocities of (a) ejections and (b) sweeps for different wallnormal positions.

6.37 Mean fluctuating velocity components within (a) ejection and (b) sweep regions for different wall-normal positions.

6.38 Root-mean-square of fluctuating velocity components of (a) ejection and (b) sweep regions for different wall-normal positions. Flow statistics are also displayed for comparison.

6.39 (a) Mean Reynolds stresses and (b) dissipation rate of ejection and sweep regions in relation to wall-normal distance. Flow statistics are also plotted for comparison. 
6.40 PDF of fluctuating streamwise, wall-normal and spanwise vorticities and magnitude of the vorticity vector at (a) ejection and (b) sweep structures for different wall-normal positions.

6.41 Root-mean-square of streamwise, wall-normal and spanwise vorticities computed in (a) ejection and (b) sweep events in relation to the distance to the wall. Flow statistics are also plotted for comparison.

6.42 Probability density function of projection angles onto the streamwise-wall-normal plane, streamwise-spanwise plane and wall-normal-spanwise plane of (a) ejection and (b) sweep regions for different wall-normal positions.

6.43 Comparison among (a) an instantaneous fluctuating velocity field and its correspondent (b) fluctuating vorticity, (c) Q-criterion and (d) $\lambda$-criterion. Vectors represents the fluctuating velocity field in the the middle plane of the tomo-PIV measurements.

6.44 PDF of (a) $Q$ and (b) $Q / Q_{\text {rms }}$ values used to detect vortices for different wall-normal distances.

6.45 Binary field after cleaning operation of the the middle plane of the tomo-PIV data using different vortex detection functions: (a) $Q / Q_{\mathrm{rms}}\left(y^{+}\right)>0.5$ and (b) $Q>2500 \mathrm{~s}^{-1}$.

6.46 Frequency of occurrence of the centre of vortical structure detected by standard and normalized Q-criterion for different wall-normal distances.

6.47 Vortex detection procedure in the the middle plane of the tomo-PIV data: (a) colorplot of $Q / Q_{\mathrm{rms}}$ and binary fields after (b) thresholding, (c) closing and opening and (d) cleaning operations.

6.48 Vortex partitioning procedure in the the middle plane of the tomo-PIV data: binary field after (a) watershed and (b) cleaning operations.

6.49 Comparison between the Q-criterion computed using (a) 9 and (b) 4 spatial derivatives. Vectors represents the fluctuating velocity field in the the middle plane of the tomo-PIV measurements.

6.50 Elevation and projection angles based on the fluctuating vorticity vector and definition of quadrants respectively on streamwise-wall-normal, streamwise-spanwise and wallnormal-spanwise planes.

6.51 PDF of (a) height, (b) width and (c) area of turbulent structures for different wall-normal positions.

6.52 Probability density function of radius in function of wallnormal distance.

6.53 (a) Mean and (b) standard deviation of radius made dimensionless by inner variables for different wall-normal positions.

6.54 (a) Mean and (b) standard deviation of radius made dimensionless by Kolmogorov length for different wall-normal positions. 
6.55 Probability density function of smallest distance between vortices as a function of wall-normal distance.

6.56 (a) Mean and (b) standard deviation of vortex separation for different wall-normal positions.

6.57 PDF of fluctuating (a) streamwise, (b) wall-normal and (c) spanwise velocities of the vortices centroid for different wall-normal positions.

6.58 (a) Mean and (b) standard deviation of fluctuating velocity components of vortex population for different wall-normal positions. Red diamond refers to flow statistics data, while dashed black lines to vortex conditional statistics.

6.59 (a) Reynold stresses and (b) dissipation rate of vortex centres in relation to wall-normal distance.

6.60 PDF of fluctuating (a) streamwise, (b) wall-normal and (c) spanwise vorticities and (d) magnitude of the vorticity vector at the centres of vortical structures for different wallnormal positions.

6.61 Root-mean-square of streamwise, wall-normal and spanwise vorticities computed for conditional and flow statistics in relation to the distance to the wall.

6.62 Probability density function of projection angles onto the (a) streamwise-wall-normal plane, (b) streamwise-spanwise plane and (c) wall-normal-spanwise plane of vortex core for different wall-normal positions.

6.63 Probability density function of elevation angles of vortex core for different wall-normal positions.

6.64 (a) Mean and (b) standard deviation of vortex streamwise inclination for different wall-normal positions.

6.65 Probability density function of projection angle on the streamwise-wall-normal plane for [Honkan and Andreopoulos, 1997] and vortex conditional statistics of tomo-PIV data.

6.66 Probability density function of elevation angle of unfiltered and filtered vortices detected on the streamwise-spanwise PIV plane at $y^{+}=110$ (adapted from figure 7 of [Ganapathisubramani et al., 2006]). The axis of the transfer function (TF) reads on the right.

6.67 Probability density function of elevation angle of unfiltered and filtered vortices detected on the wall-normal-spanwise plane at $100<y^{+}<500$ at the middle plane of tomo-PIV data. The axis of the transfer fuction (TF) reads on the right. 183

6.68 Probability density function of elevation angle of unfiltered and filtered vortices detected on the wall-normal-spanwise plane at $100<y^{+}<500$ at the middle plane of tomo-PIV data. The axis of the transfer fuction (TF) reads on the right.

7.1 Square water channel sketch.

7.2 (a) Upstream and (b) downstream pictures of the water channel.191

7.3 Plenum chambers at (a) inlet and (b) outlet of the water channel.191 
7.4 Water-channel modulus of the tomo-PIV testing zone showing the detail of the squared cross-section.

7.5 Sample pictures of the camera calibration target imaged by (a) camera 1 and (b) camera 2 employing (bottom) and not employing (upper) prisms, for the same camera configuration.

7.6 Schematic views of camera arrangement in the water-channel experiment.

7.7 Top view picture of the camera arrangement in the waterchannel test section showing the Scheimpflug configuration.

7.8 Tomo-PIV experimental acquisition showing (a) upstream and (b) downstream part of the optical path in the water channel.

7.9 Sample of tomo-PIV particle images recorded by camera 2 employing a laser illumination coming (a) from the side and (b) from the top walls of the water channel.

7.10 Zoom of an area of 160x160 pixels of a particle image sample from camera 2.

7.11 Sample of particle images of the water-channel tomo-PIV experiment recorded by camera 3 . Particle concentration of (a) $0.004 \mathrm{ppp}\left(N_{s}=0.078\right)$ and (b) $0.010 \mathrm{ppp}\left(N_{s}=0.196\right)$.

7.12 (a) Detail of the transparent target of dot pattern fixed on the stainless-steel frame. (b) Transparent target on its support.

7.13 Schematic isometric view of the transverse structure supporting the cameras in the water-channel experiment.

202

7.14 (a) Target alignment with the laser fat sheet at $z^{*}=0$.

(b) Recording procedure of target images with background illumination.

7.15 Images of the target placed inside the channel at $z^{*}=0$ position for each camera. The images show the image coordinate system (in red), the world coordinate system (in purple) and the channel coordinate system (in white). In order to enhance the visualization the third axis from the world and channel systems can be suppressed sometimes.

7.16 2D disparity map of camera 1 in the middle of the laser sheet for self-calibration method with a search radius of 7 pixels.

7.17 Amplified upper-left region of the middle-plane disparity maps for search radios of 7 pixel before self-calibration correction.

7.18 2D disparity map of camera 1 in the middle of the laser sheet for self-calibration method with a search radius of 3 pixels.

7.19 Amplified upper-left region of the middle-plane disparity maps for search radios of 3 pixel after self-calibration correction.

8.1 Sample of (a) raw and (b) pre-processed images from camera 1 in the water-channel experiment.

8.2 Sample of particle intensity inside the tomo-PIV reconstructed volume.

8.3 Sample of an instantaneous normalized intensity profile of the reconstructed volume for laser pulses A and B. 
8.4 Sample of 3D tomo-PIV velocity field in the water-channel experiment. The color code refers to the streamwise velocity. One over three 2D vectors of wall-normal and spanwise velocity components are also plotted.

8.5 Six consecutive instantaneous velocity fields at the middle plane of tomo-PIV measurements. Colour code related to the instantaneous stream-wise velocity and vectors related to the wall-normal and spanwise instantaneous velocity components.

8.6 Six consecutive fluctuating velocity fields at the middle plane of tomo-PIV measurements. Colour code related to the fluctuating stream-wise velocity and vectors related to the wallnormal and spanwise fluctuating velocity components.

8.7 Six consecutive fluctuating vorticity fields at the middle plane of tomo-PIV measurements. Colour code related to the fluctuating stream-wise vorticity and vectors related to the wallnormal and spanwise fluctuating vorticity components.

Six consecutive fluctuating velocity fields at the middle plane of tomo-PIV measurements. Colour code related to the 3D Q-criterion and vectors related to the wall-normal and spanwise fluctuating velocity components.

8.9 Interaction among low-speed streaks (green isosurfaces of $u=0.32 \mathrm{~m} / \mathrm{s}$ ) and turbulent vortical structures (blue isosurfaces of Q-criterion $=500 \mathrm{~s}^{-1}$ ) inside a water channel from tomo-PIV filtered data.

8.10 (a) Mean streamwise velocity at the middle of the volume. Channel walls were located at $y=0, \quad y=40, z=-20$ and $z=20 \mathrm{~mm}$. (b) Contour lines of the primary mean streamwise flow, and vectors of the secondary mean wall-normal and spanwise flows for the DNS of [Vinuesa et al., 2014]. Red rectangle refers to a similar region of the present tomo-PIV study.

8.11 Comparison among mean streamwise velocity profiles at the channel centre for tomo-PIV measurements and classical laminar and power-law curves.

8.12 Profiles of mean streamwise velocity in (a) semi-log and (b) linear plots in inner scales.

8.13 Profiles of turbulent velocity fluctuations dimensionless by half-height of the channel. Comparison with DNS of a channel flow from [Moser et al., 1999].

8.14 Profiles of divergence and uncertainty in velocity.

8.15 Probability density functions at $y^{+}=50$ for (a) $u^{\prime} / \sqrt{\left\langle u^{\prime 2}\right\rangle}$, (b) $v^{\prime} / \sqrt{\left\langle v^{\prime 2}\right\rangle}$ and (c) $w^{\prime} / \sqrt{\left\langle w^{\prime 2}\right\rangle}$ in the water-channel experiment. 


\section{List of Tables}

2.1 Properties of wall regions and layers (adapted from [Pope, 2000]). 38

4.1 Camera parameters for the boundary-layer tomo-PIV experiment. 78

4.2 Image parameters for the boundary-layer tomo-PIV experiment. 78

4.3 Average particle density per pixel for each experimental run. 82

4.4 Evolution of the projection and the triangulation errors of each camera during the translated-plane self-calibration corrections for run 14 (in pixel).

4.5 Projection and the triangulation errors of the camera calibrations for each run (in pixel).

5.1 Camera calibration, image pre-processing, tomographic reconstruction algorithm and cross-correlation details for the present work and Davis (LaVision).

6.1 Tomo-PIV reconstructed volume characteristics. 118

6.2 Tomo-PIV velocity field characteristics. 118

$\begin{array}{lll}6.3 & \text { Boundary-layer characteristics. } & 122\end{array}$

7.1 Camera parameters. 194

7.2 Average amount of particles in each camera for the waterchannel tomo-PIV experiment. 200

7.3 Image parameters. 201

7.4 Projection and triangulation errors of each camera before and

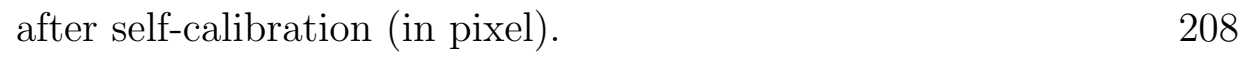

8.1 Tomo-PIV characteristics of the water-channel experiment. 213

8.2 Water-channel flow characteristics. 213 


\section{List of Latin Symbols}

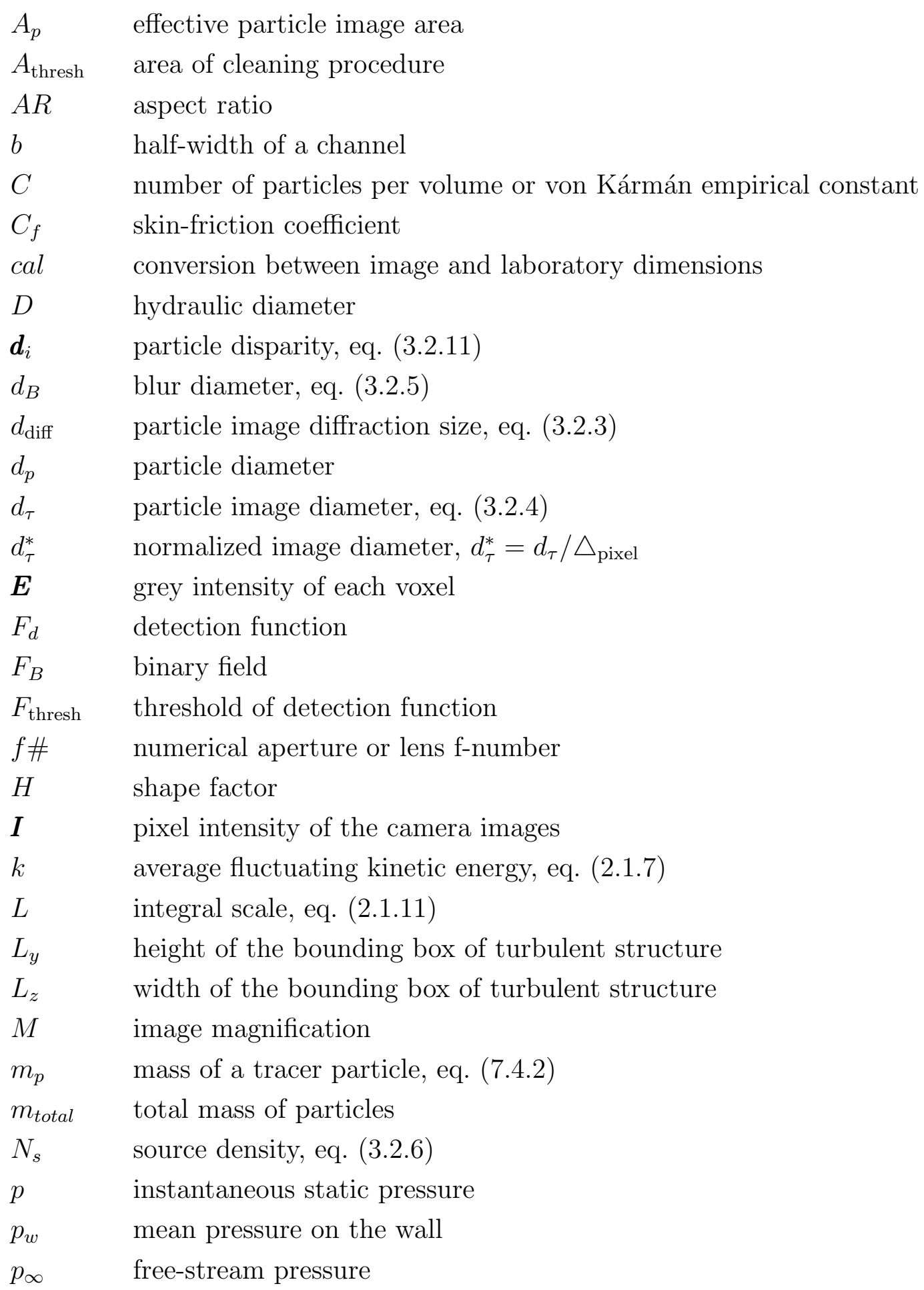




\begin{tabular}{|c|c|c|}
\hline & ppp & particles per pixel \\
\hline & $p p v$ & particles per volume \\
\hline & $Q_{\text {rec }}$ & standard reconstruction quality, eq. (3.3.1) \\
\hline & $Q_{\text {reproj }}$ & re-projection quality, eq. (3.3.2) \\
\hline & $Q_{2 D}$ & Q-criterion based on 4 spatial derivatives \\
\hline & $Q_{3 D}$ & Q-criterion based on 9 spatial derivatives \\
\hline & $r$ & radius of turbulent structure \\
\hline & $R$ & cross-correlation coefficient \\
\hline & $s_{i j}$ & instantaneous strain rate tensor, eq. (2.1.3) \\
\hline & $\operatorname{Re}_{D}$ & Reynolds number based on the hydraulic diameter, eq. (2.3.1) \\
\hline & $\operatorname{Re}_{x}$ & Reynolds number based on the streamwise position, eq. (2.2.4) \\
\hline & $\operatorname{Re}_{\delta}$ & Reynolds number based on the boundary-layer thickness, eq. (2.2.4) \\
\hline & $\operatorname{Re}_{\delta^{*}}$ & Reynolds number based on the displacement thickness, eq. (2.2.4) \\
\hline & $\operatorname{Re}_{\theta}$ & Reynolds number based on the momentum thickness, eq. (2.2.4) \\
\hline & $R e_{\tau}$ & Reynolds number based on the viscous length scales \\
\hline & S & symmetric component of the velocity gradient tensor \\
\hline$\stackrel{\varrho}{\varrho}$ & $S N R_{E}$ & light intensity signal-to-noise ratio, eq. (3.3.4) \\
\hline & $S N R_{N}$ & particle distribution signal-to-noise ratio, eq. (3.3.3) \\
\hline$\frac{N}{1}$ & $t$ & time \\
\hline$\frac{L}{\pi}$ & $u$ & instantaneous streamwise velocity or $\mathrm{x}$-axis of the image system \\
\hline . & $u_{i}^{\prime}$ & $i$-th component of the instantaneous velocity \\
\hline זָָ & $U$ & mean streamwise velocity \\
\hline $\bar{E}$ & $u^{\prime}$ & fluctuating streamwise velocity \\
\hline 㐫 & $u_{i}^{\prime}$ & $i$-th component of the fluctuating velocity \\
\hline$\stackrel{\circ}{r}$ & $U_{\max }$ & maximum mean velocity inside the duct \\
\hline ِ & $\mathrm{u}_{\eta}$ & Kolmogorov time scale, eq. (2.1.9) \\
\hline & $u_{\tau}$ & friction velocity, eq. (2.2.9) \\
\hline & $U_{\infty}$ & free-stream velocity \\
\hline & $\bar{U}$ & bulk velocity, eq. (2.3.2) \\
\hline & $v$ & instantaneous wall-normal velocity or y-axis of the image system \\
\hline & $V$ & mean wall-normal velocity \\
\hline & $v^{\prime}$ & fluctuating wall-normal velocity \\
\hline & $V_{v o l}$ & volume of water inside the channel \\
\hline & $w$ & $\begin{array}{l}\text { instantaneous spanwise velocity or voxel weight of reconstruction } \\
\text { algorithms }\end{array}$ \\
\hline & $w^{\prime}$ & fluctuating spanwise velocity \\
\hline & $W$ & mean spanwise velocity \\
\hline
\end{tabular}




$\begin{array}{ll}x & \text { streamwise direction } \\ x^{*} & \text { x-axis of the world system } \\ x_{1} & \text { streamwise direction } \\ x_{2} & \text { wall-normal direction } \\ x_{3} & \text { spanwise direction } \\ y & \text { wall-normal direction } \\ y^{*} & \text { y-axis of the world system } \\ y_{0} & \text { position of the turbulent structure centre } \\ z & \text { spanwise direction } \\ z^{*} & \text { z-axis of the world system }\end{array}$

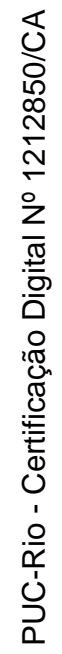




\section{List of Greek Symbols}

\begin{tabular}{|c|c|c|}
\hline & $\alpha$ & relaxation factor \\
\hline & $\delta$ & boundary-layer thickness or half-height of the channel \\
\hline & $\delta^{*}$ & displacement thickness, eq. (2.2.2) \\
\hline & $\delta_{i j}$ & Kronecker delta \\
\hline & $\delta_{\boldsymbol{u}}$ & uncertainty in velocity measurements, eq. (3.3.9) \\
\hline & $\delta u^{\prime}$ & uncertainty in the fluctuating velocity \\
\hline & $\delta_{z}$ & focal depth, eq. (3.2.1) \\
\hline & $\delta_{\nu}$ & viscous length scale, eq. (2.2.10) \\
\hline & $\delta \omega^{\prime}$ & uncertainty in fluctuating vorticity \\
\hline & $\triangle_{\text {pixel }}$ & pixel size \\
\hline & $\triangle t$ & time interval between two pulses \\
\hline & $\triangle_{\text {vec }}$ & vector spacing \\
\hline & $\triangle x$ & streamwise length \\
\hline Oֵ & $\triangle y$ & wall-normal length \\
\hline 党 & $\triangle z$ & spanwise length or illuminated volume thickness \\
\hline$\frac{1}{2}$ & $\epsilon$ & rate of dissipation of turbulence kinetic energy, eq. (2.1.8) \\
\hline$\frac{2}{\pi}$ & $\varepsilon_{B}$ & distance between the particle and the focal plane \\
\hline 置 & $\varepsilon_{y}$ & error in wall-normal position \\
\hline ז్ֶ & $\epsilon_{\boldsymbol{x}}$ & error in particle position, eq. (3.3.7) \\
\hline 0 & $\epsilon_{\boldsymbol{u}}$ & error in velocity measurements, eq. (3.3.8) \\
\hline$\bigcup_{1}^{0}$ & $\eta$ & Kolmogorov length scale, eq. (2.1.9) \\
\hline$\frac{\mathrm{o}}{\mathrm{r}}$ & $\theta$ & momentum thickness, eq. (2.2.3) \\
\hline بـ & $\theta_{e}$ & elevation angle \\
\hline & $\theta_{x}$ & angle with respect to the wall-normal-spanwise plane \\
\hline & $\theta_{x y}$ & $\begin{array}{l}\text { projection angles with respect to the streamwise-wall-normal plane, } \\
\text { eq. }(6.2 .34)\end{array}$ \\
\hline & $\theta_{x z}$ & $\begin{array}{l}\text { projection angles with respect to the streamwise-spanwise plane, } \\
\text { eq. }(6.2 .35)\end{array}$ \\
\hline & $\theta_{y}$ & angle with respect to the streamwise-spanwise plane \\
\hline & $\theta_{y z}$ & $\begin{array}{l}\text { projection angles with respect to the wall-normal-spanwise plane, } \\
\text { eq. }(6.2 .36)\end{array}$ \\
\hline & $\theta_{z}$ & angle with respect to the streamwise-wall-normal plane \\
\hline
\end{tabular}




\begin{tabular}{|c|c|c|}
\hline & $\kappa$ & von Kármán empirical constant \\
\hline & $\lambda$ & wavelength of light or Taylor micro-scale \\
\hline & $\lambda_{c i}$ & swirling strength criterion \\
\hline & $\lambda_{c i 2 D}$ & swirling strength criterion in a plane \\
\hline & $\lambda_{c i 3 D}$ & swirling strength criterion in a volume \\
\hline & $\lambda_{f}$ & Taylor micro-scale, eq. (2.1.10) \\
\hline & $\lambda_{22 D}$ & $\lambda_{2}$-criterion in a plane \\
\hline & $\lambda_{23 D}$ & $\lambda_{2}$-criterion in a volume \\
\hline & $\mu$ & dynamic viscosity or relaxation parameter \\
\hline & $\nu$ & kinematic viscosity of the fluid, $\nu \equiv \mu / \rho$ \\
\hline & $\xi$ & generic flow quantity \\
\hline & $\rho$ & fluid density \\
\hline & $\sigma$ & average of standard deviations \\
\hline & $\sigma_{E}^{*}$ & normalized intensity variance, eq. (3.3.5) \\
\hline & $\tau$ & total shear stress \\
\hline & $\tau_{i j}$ & viscous stress tensor \\
\hline & $\tau_{w}$ & shear stress at the wall, eq. (2.2.7) \\
\hline & $\tau_{\eta}$ & Kolmogorov velocity scale, eq. (2.1.9) \\
\hline & $\Phi$ & mapping function, eq. (3.2.8) \\
\hline$\frac{2}{\pi}$ & $\Phi_{x y}$ & $\begin{array}{l}\text { projection angles with respect to the streamwise-wall-normal plane, } \\
\text { eq. }(6.2 .16)\end{array}$ \\
\hline 雵 & $\Phi_{x z}$ & $\begin{array}{l}\text { projection angles with respect to the streamwise-spanwise plane, } \\
\text { eq. }(6.2 .17)\end{array}$ \\
\hline & $\Phi_{y z}$ & $\begin{array}{l}\text { projection angles with respect to the wall-normal-spanwise plane, } \\
\text { eq. }(6.2 .18)\end{array}$ \\
\hline & $\omega_{x}$ & instantaneous streamwise vorticity \\
\hline & $\omega_{x}^{\prime}$ & fluctuating streamwise vorticity \\
\hline & $\omega_{y}$ & instantaneous wall-normal vorticity \\
\hline & $\omega_{y}^{\prime}$ & fluctuating wall-normal vorticity \\
\hline & $\omega_{z}$ & instantaneous spanwise vorticity \\
\hline & $\omega_{z}^{\prime}$ & fluctuating spanwise vorticity \\
\hline & $\Omega$ & antisymmetric component of the velocity gradient tensor \\
\hline
\end{tabular}


Turbulence remains the last great unsolved problem of classical Physics.

Richard Feynman, Nobel laureate in Physics. 


\section{1}

\section{Introduction}

The understanding of turbulent behaviour in flowing fluids is one of the most important problems in classical physics. Furthermore, most fluid flows are turbulent and turbulence, in many cases, is the dominant physics that controls other processes. Turbulent flows are found in circulatory system of living organisms, flowing rivers, atmospheric wind, astrophysical movement, pipeline transportation, vehicles and countless other practical engineering applications ([McDonough, 2007]). Turbulent flows, close to the wall, can impose a resistance of about $50 \%$ (surface friction related to total resistance) for ordinary civil airplanes, $70 \%$ for submarines and it can be related to the total resistance for long-distance pipeline transportation ([Wang et al., 2000]). A slight reduction of these surface friction values, by optimization or successful control, can result in substantial savings of energy sources and money.

Turbulent flows are three-dimension and unsteady, with rapid variations of flow properties. In addition, this type of flow contains eddy structures spread over a very wide range of sizes, which make a mathematical description of turbulence a complex task and the prediction of turbulent behaviour challenging ([McDonough, 2007]). In the literature, there are many definitions of turbulence, dating as early as the $16^{\text {th }}$ Century with the first qualitative observations of Leonardo da Vinci. In his study, the movement of turbulent flows was illustrated detailing eddies behaviour of various length ranges (Codex Atlanticus). In the last century, [Richardson, 1922] introduced the concept of "energy cascade", a process in which the energy injected into a fluid at the large scales of length and time is transferred to successively smaller scales until it is finally dissipated at molecular scales. From another perspective, [Von Kármán, 1938] emphasized the chaotic nature of turbulence. An additional approach was proposed taking into account that distinct average quantities of turbulence, such as velocity, pressure and temperature, can also be obtained by the theory of probability, despite the irregular motion of turbulent fluid flow (e.g., [Hinze, 1975]). [Chapman and Tobak, 1985] summarized the turbulence understanding in terms of statistical, structural and deterministic definitions as displayed in the chart of figure 1.1. For these authors, turbulence is any chaotic solution 


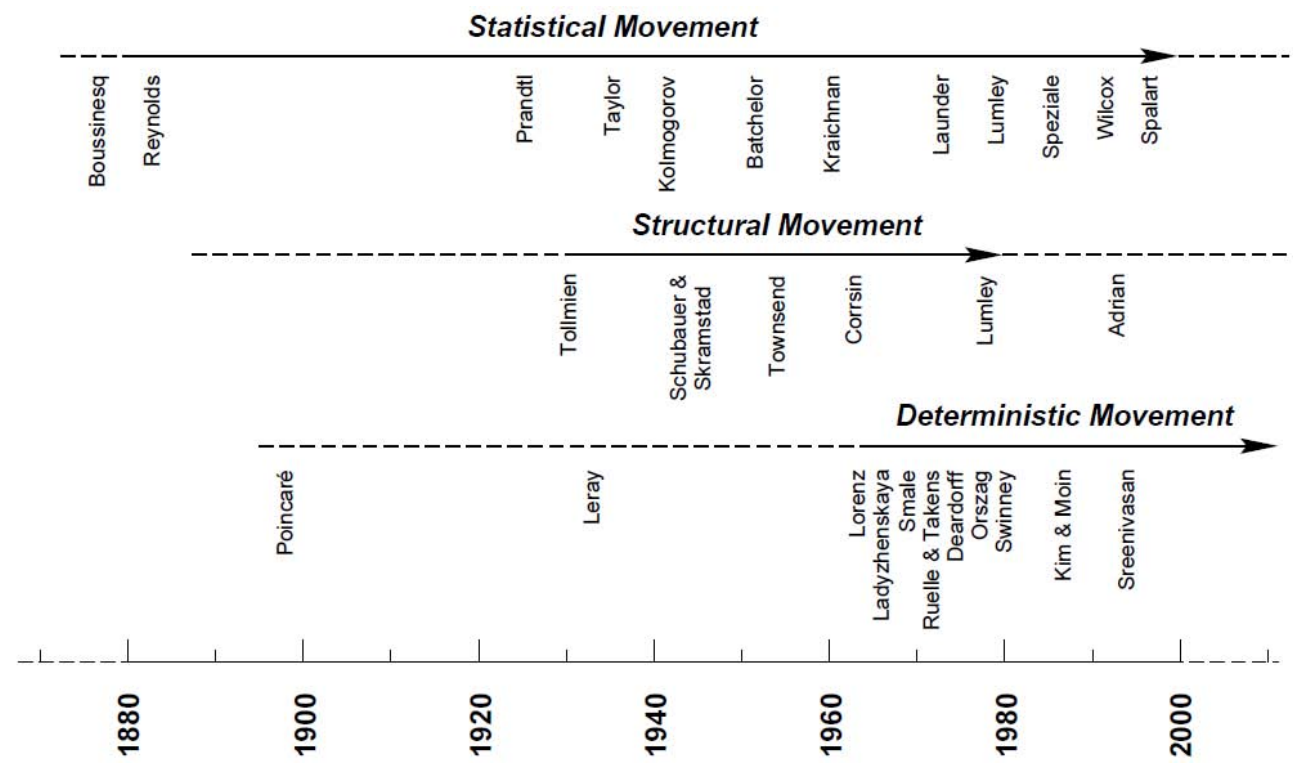

Figure 1.1: Movements in the study of turbulence ([McDonough, 2007]).

to the 3-D Navier-Stokes equations that is originated from the stability loss of the laminar flow regime due to initial perturbations.

Despite the widespread occurrence of turbulent fluid flows and the effort of many authors to explain the phenomena involved, the turbulence problem remains not understood in complete detail. In order to organize this complex field, the study of turbulence was divided into two main categories ([Hinze, 1975]): "free turbulence", referred to the turbulence in the absence of walls and "wall turbulence", related to the turbulence generated and affected by the walls, with the latter being the focus of the present work.

Nowadays, it seems to be a consensus that the wall-turbulence flow is not entirely stochastic but formed by organized structures. In the near-wall region, a population of many eddies of different scales interacts with one another in a complex phenomenon of a continuous self-sustaining process. These eddies can present quasi-periodic patterns of coherent motion in the flow that seems to be responsible for the maintenance of turbulence. Despite the fact that there are numerous works on this subject (e.g., [Theodorsen, 1952, Kline and Runstadler, 1958, Kline et al., 1967, Schlichting, 1979, Hinze, 1975, Robinson, 1991b, Panton, 1997, Schoppa and Hussain, 2002, Adrian et al., 2000b], among others), the near-wall turbulence structures, their evolution and their interplay remain not fully understood ([Stanislas et al., 2008, Foucaut et al., 2011b]).

The construction of turbulence knowledge was boosted by the evolution of computers and equipments that changed the way of analysing the flow. Turbulence measurements achieved a notorious improvement with the advances in Particle Image Velocimetry (PIV) ([Raffel et al., 2007]). Nowadays, variations 


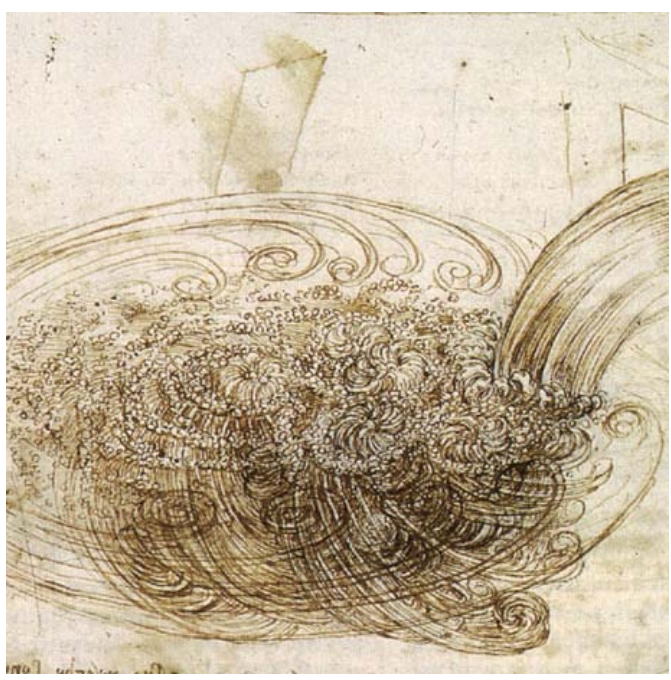

(a)

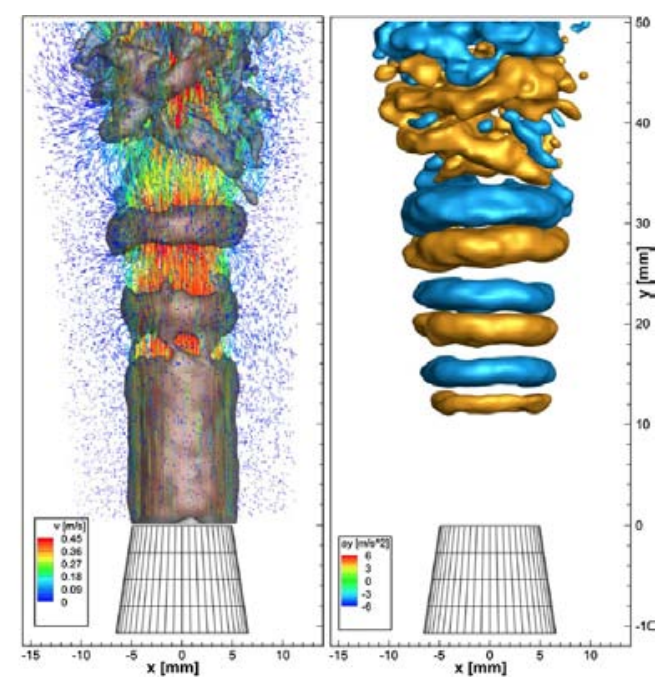

(b)

Figure 1.2: (a) Sketch of the turbulent water motion by Leonardo da Vinci (Codex Atlanticus), showing the unsteady eddying motions. (b) Middle-cut volume through a transitional jet calculated by PTV-STB and visualized by FlowFit (adapted from [Schanz et al., 2016]). Instantaneous isosurfaces of vorticity with superimposed particle tracks, extending 5 time-steps back (left). Isosurfaces of streamwise acceleration at the same time-step (right).

of this non-intrusive technique are able to capture the full three-dimensionality of unsteady flows by means of multi-plane PIV ([Kähler and Kompenhans, 2000]), three-dimensional particle tracking velocimetry (3D PTV) ([Maas et al., 1993]), defocusing PIV ([Pereira et al., 2000]), holographic and digital holographic PIV (HPIV and DHPIV) ([Schnars and Jüptner, 1994]), scaning-PIV ([Hori and Sakakibara, 2004]), tomo-PIV ([Elsinga et al., 2006]) and recently PTV Shake The Box (STB) ([Schanz et al., 2013b]). Figure 1.2 exemplifies this evolution, showing side-by-side the artistic sketch of the pioneer Leonardo da Vinci and time-resolved quantitative measurements applying the state-of-the-art PTV-STB technique.

The present work employed the tomo-PIV measurement technique, which can provide 3D data of turbulent flow structures, to contribute to the understanding of wall turbulence. Tomo-PIV is based on the volume reconstruction of the image of illuminated particles. The flow is seeded by tracer particles that are twice illuminated by a light source, similarly to standard PIV. The light scattered from the particles is recorded in pairs of images by distinctive camera views. The instantaneous flow is estimated by $3 \mathrm{D}$ cross-correlation of a pair of reconstructed volumes computed from these views. Some reconstruction methods ([Scarano, 2013]) were proposed to solve this under-determined system composed by particle distribution from a limited number of views. Unfortunately, the volume reconstruction with limited views produces a larger 
number of particles, as compared to those actually present in the illuminated volume ([Maas et al., 1993]). These extra particles can increase bias and random errors in velocity measurements ([Novara et al., 2010, de Silva et al., 2013, Elsinga and Tokgoz, 2014]). Thus, the performance and accuracy of this technique is highly dependent on the parametric adjustment of the image and the reconstruction algorithm used. Despite the fact that synthetic data have been extensively used to optimize experiments, the use of these parameters in actual flow experiments may not result in reconstructions with optimal quality. Therefore, the mentioned errors must be minimized by optimization of the actual tomo-PIV experiment and tomo-processing.

The present work was carried out in the framework of a joint doctoral program involving the Laboratoire de Mécanique de Lille (LML), in France, and the Mechanical Engineering Department of PUC-Rio, in Brazil. In the first part of the research, conducted at the LML wind tunnel facility, the unsteady character of a near-wall boundary layer flow over a flat plate was analysed based on particle image data obtained from a previously conducted experiment ([Foucaut et al., 2011a]). These data sets were recorded from a 6-camera, high-repetition, tomo-PIV experiment viewing a spanwise-wall-normal thin volume in the vicinity of the tunnel wall. The second part of the experiments, conducted at the Department of Mechanical Engineering of PUC-Rio, involved the design, construction and optimization of a square-channel, turbulent water flow experiment. The focus of the study was on the analysis of near-wall structures in channel flow. For this experiment, a 4-camera high-repetition tomo-PIV was employed, viewing a spanwise-wall-normal thin volume.

\subsection{Objective}

The objective of the present work was to study near-wall turbulent flows over a smooth flat plate in a wind tunnel and inside a square water channel. For this purpose, the non-intrusive tomo-PIV was used. This technique measures 4-dimensions and 3-components (4D-3C) velocity fields, enabling the computation of the full velocity gradient tensor and the reconstruction of the time history of the turbulent structures. Then, coherent structures can be identified, in order to obtain its mean characteristics, spatial distribution, as well as the interactions among turbulent structures.

Since the performance and accuracy of tomo-PIV are conditioned to the adjustment of the parameters of the technique and the reconstruction algorithm employed, a second objective of the work was to optimize the tomo-PIV processing. For the flat-plate near-wall study, once the image particle distribution data have been already acquired, the optimization effort was 
focused on image pre-processing, volume reconstruction, cross-correlation of reconstructed volumes and post-processing of calculated velocity fields. For the water channel flow studies, besides the aforementioned optimization, the full tomo-PIV experiment was optimized by remodelling the channel test section and setting the volume illumination, particle seeding, camera arrangement and image acquisition.

An additional objective of the present study was to implement a 4D-3C data post-processing tool to aid in the study of near-wall turbulence. To this end, a software tool was developed to extract statistics of the flow and of the turbulent structures obtained from the tomo-velocity fields, displaying them as 2D and 3D plots to aid in the understanding of the results and, consequently, contributing to the study of the near-wall turbulent flow phenomena.

It is worth mentioning that the transfer of the knowledge on the tomoPIV technique to the Brazilian research community is a relevant objective of the present work. To the best knowledge of the author, this is the first implementation of a tomo-PIV experiment in the country. 


\section{2}

\section{Wall Turbulence}

This chapter makes a brief introduction about turbulence equations, followed by the description of important physical quantities in boundary layer and channel flows. Then, a review of near-wall turbulent structures, so important in the turbulent self-sustaining process, is presented.

\subsection{Brief Introduction to Turbulence}

This section introduces some relevant equations for the understanding of the turbulent phenomenon. Further explanations can be found in the books of [Mathieu and Scott, 2000] and [Pope, 2000]. The Cauchy momentum equations, which are now a consensus to describe the physics of all fluid flows that proprieties do not vary at molecular scale, are expressed as

$$
\rho\left[\frac{\partial u_{i}}{\partial t}+u_{j} \frac{\partial u_{i}}{\partial x_{j}}\right]=-\frac{\partial p}{\partial x_{i}}+\frac{\partial \tau_{i j}}{\partial x_{j}},
$$

where $\rho$ is the fluid density, $u_{i}$ the $i$-th component of the instantaneous fluid velocity at the position $\left(x_{1}, x_{2}, x_{3}, t\right), t$ time, $p$ the instantaneous static pressure and $\tau_{i j}$ the viscous stress tensor. Along the text $x_{1}$ or just $x$ denotes the streamwise, $x_{2}$ or $y$ the wall-normal and $x_{3}$ or $z$ the spanwise directions. The equations are written using the Cartesian tensor notation and the Einstein summation convention. Sometimes along the text $u, v$ and $w$ can be used in reference to the streamwise, wall-normal and spanwise velocity, respectively.

The momentum equations are the Newton's second law that relates the rate of change of momentum per unit mass to the contact and body forces ([George, 2013]). They are linked to the continuity equation

$$
\left[\frac{\partial \rho}{\partial t}+u_{j} \frac{\partial \rho}{\partial x_{j}}\right]+\rho \frac{\partial u_{j}}{\partial x_{j}}=0 .
$$

Assuming a Newtonian fluid, eq. (2.1.1) becomes the Navier-Stokes momentum equations. Adding the hypothesis of incompressible fluid, the viscous stress simplifies to $\tau_{i j}=2 \mu s_{i j}$, where $\mu$ is the dynamic viscosity and $s_{i j}$ is the instantaneous strain rate tensor given by 


$$
s_{i j}=\frac{1}{2}\left[\frac{\partial u_{i}}{\partial x_{j}}+\frac{\partial u_{j}}{\partial x_{i}}\right]
$$

Using the Reynolds decomposition into mean and fluctuating part of turbulent quantities ([Pope, 2000]), the averaged momentum equations for an incompressible fluid become

$$
\rho\left[\frac{\partial<u_{i}>}{\partial t}+<u_{j}>\frac{\partial<u_{i}>}{\partial x_{j}}\right]=-\frac{\partial<p>}{\partial x_{i}}+\frac{\partial}{\partial x_{j}}\left[<\tau_{i j}>-\rho<u_{i}^{\prime} u_{j}^{\prime}>\right],
$$

where $\left\langle u_{i}\right\rangle$ is the $i$-th component of the time-averaged velocity, $u_{i}^{\prime}$ is the $i$ th component of the fluctuating velocity, $\langle p\rangle$ is the time-averaged pressure, $<\tau_{i j}>$ the viscous stress of time-averaged velocity components and $\rho<u_{i}^{\prime} u_{j}^{\prime}>$ the Reynolds stress tensor. The latter expresses the fluctuating contribution to the non-linear acceleration terms.

Considering the previous assumptions, plus the hypothesis of constant and homogeneous fluid density for the mass conservation, eq. (2.1.2) results in divergence of velocity equal to zero.

$$
\frac{\partial<u_{i}>}{\partial x_{i}}=0 \quad \text { and } \quad \frac{\partial u_{i}^{\prime}}{\partial x_{i}}=0
$$

After some manipulations of the Navier-Stokes equations, the equation for the average kinetic energy per unit mass of the fluctuating motion is obtained

$$
\begin{gathered}
\frac{\partial}{\partial t}{ }^{1}+<u_{j}>\frac{\partial}{\partial x_{j}} k=-<u_{i}^{\prime} u_{j}^{\prime}>\frac{\partial<u_{i}>}{\partial x_{j}} \\
-2 \nu<s_{i j} s_{i j}>+\frac{\partial}{\partial x_{j}}\left[-\frac{1}{\rho}<p u_{i}^{\prime}>\delta_{i j}-\frac{1}{2}<q^{2} u_{j}^{\prime}>+2 \nu<s_{i j} u_{i}^{\prime}>\right]
\end{gathered}
$$

where $\delta_{i j}$ is the Kronecker delta, $\nu \equiv \mu / \rho$ the kinematic viscosity of the fluid, $q^{2} \equiv u_{i}^{\prime} u_{i}^{\prime}$ and $k$ the average fluctuating kinetic energy given by

$$
k \equiv \frac{1}{2}<u_{i}^{\prime} u_{i}^{\prime}>=\frac{1}{2}\left[<u_{1}^{\prime 2}>+<u_{2}^{\prime 2}>+<u_{3}^{\prime 2}>\right] .
$$

These kinetic energy equation can be explored for understanding the dynamics of turbulent motion. Each term in the eq. (2.1.6), numbered from 1 to 5 , has a distinct role in the overall kinetic energy balance as described in [George, 2013].

1. Rate of change of kinetic energy per unit mass due to non-stationarity. 
2. Rate of change of kinetic energy per unit mass due to convection by the mean flow through an inhomogeneous field.

3. Rate of production of turbulence kinetic energy from the mean flow gradient. Almost always the kinetic energy is removed from the mean to the fluctuating motions, however this term can be positive or negative characterizing the kinematic energy interchange.

4. Rate of dissipation of turbulence kinetic energy per unit mass due to viscous stresses. Energy is dissipated because of the work of fluctuating viscous stresses in resisting deformation of the fluid material by the fluctuating strain rates. This is a one-way process and kinetic energy is decreased while internal energy is increased.

5. Transport of kinetic energy in an inhomogeneous field due, respectively, to the pressure fluctuations, the turbulence itself, and the viscous stresses. This terms characterize the energy movement from one place to another, neither creating nor destroying it in the process.

The rate of dissipation of turbulence kinetic energy by viscous stresses, for a Newtonian incompressible fluid, simplifies to ([Foucaut et al., 2016])

$$
\epsilon \equiv \frac{\nu}{2}\left\langle\left[\frac{\partial u_{i}}{\partial x_{j}}+\frac{\partial u_{j}}{\partial x_{i}}\right]^{2}\right\rangle
$$

Simulations and experimental observations of turbulent flows supported the Kolmogorov theory that proposes that micro-scale eddies dissipate the kinetic energy of the large eddies into heat in the cascade process of energy transfer ([George, 2013]). As a result, the statistics of the small-scale motions are universal and the small-scale eddies depend only on the dissipation rate $\epsilon$ and the kinematic viscosity $\nu$. The Kolmogorov length, time and velocity scales, representative of dissipation eddies, are defined, respectively, as

$$
\eta \equiv\left[\frac{\nu^{3}}{\epsilon}\right]^{1 / 4} \quad, \quad \mathrm{u}_{\eta} \equiv[\nu \epsilon]^{1 / 4} \quad, \quad \tau_{\eta} \equiv\left[\frac{\nu}{\epsilon}\right]^{1 / 2} .
$$

In the inertial sub-range ([Pope, 2000]), eddies of the size of Taylor microscale dominate. This scale can be computed by the osculating parabola from the autocorrelation function of streamwise velocity ([George, 2013]). The peak of this parabola coincides with the peak of the autocorrelation function in the ordinate axis and the intersection of the former curve with the abscissa axis gives the Taylor micro-scale value. For isotropic turbulence, Taylor length scale can also be defined, according to [Pope, 2000], as 


$$
\lambda_{f} \equiv \sqrt{\frac{20 \nu k}{\epsilon}}
$$

Length scale characterizing the large eddies ([Pope, 2000]), which contain the most part of the flow energy, is given by

$$
L \equiv \frac{k^{3 / 2}}{\epsilon}
$$

\subsection{Boundary Layer Flow}

A boundary layer is the layer of fluid in the immediate vicinity of a bounding surface where the effects of viscosity are significant. This section describes important physical quantities of turbulent boundary layers. Further details can be found in [Schlichting and Gersten, 2000]. The free streamvelocity outside the boundary layer is denoted by $U_{\infty}(x)$. The surface of the wall is located at $y=0$ for $x \geq 0$, where $x$ is the streamwise, $y$ the wall-normal and $z$ span-wise direction. The mean flow is mainly in the streamwise direction and is statistically two-dimensional in the $(x, y)$ plane.

The free-stream pressure $p_{\infty}(x)$ is related to the free-stream velocity $U_{\infty}(x)$ through Euler's equation.

$$
-\frac{\mathrm{d} p_{\infty}}{\mathrm{d} x}=\rho U_{\infty} \frac{\mathrm{d} U_{\infty}}{\mathrm{d} x}
$$

Accelerating flow $\mathrm{d} U_{\infty} / \mathrm{d} x>0$ corresponds to a favourable pressure gradient, and $\mathrm{d} U_{\infty} / \mathrm{d} x<0$ to an adverse pressure gradient, which can eventually lead the separation of the boundary layer from the surface. In the simpler zero-pressure-gradient case, $U_{\infty}(x)$ is constant.

The boundary-layer thickness $\delta(x)$, which develops continuously and increases in the flow direction, is defined as the $y$ position where the mean streamwise velocity $\langle u\rangle(x, y)$ or $U(x, y)$ corresponds to $0.99 U_{\infty}(x)$.

The uncertainty in the $\delta(x)$ measurement is high due to the fact that it is based on small velocity differences. Conversely, other thickness characterizing the boundary layer were proposed based on integral measures, namely displacement thickness,

$$
\delta^{*}(x) \equiv \int_{0}^{\delta}\left[1-\frac{<u>}{U_{\infty}}\right] \mathrm{d} y \quad,
$$

and momentum thickness,

$$
\theta(x) \equiv \int_{0}^{\delta} \frac{<u>}{U_{\infty}}\left[1-\frac{<u>}{U_{\infty}}\right] \mathrm{d} y
$$


The Reynolds number, which expresses the relative importance of inertial and viscous forces in the fluid flow, can be defined based on the streamwise position or these boundary-layer thicknesses

$$
\operatorname{Re}_{x} \equiv \frac{U_{\infty} x}{\nu} \quad, \quad \operatorname{Re}_{\delta} \equiv \frac{U_{\infty} \delta}{\nu} \quad, \quad \operatorname{Re}_{\delta^{*}} \equiv \frac{U_{\infty} \delta^{*}}{\nu} \quad, \quad \operatorname{Re}_{\theta} \equiv \frac{U_{\infty} \theta}{\nu}
$$

The flatness of the mean streamwise velocity profile can be characterized by the ratio between the displacement and momentum thicknesses, defined as shape factor $H \equiv \delta^{*}(x) / \theta(x)$.

The governing Navier-Stokes equations can be simplified within the boundary layer using an order of magnitude analysis and eq. (2.2.1) ([Pope, 2000]). The mean streamwise momentum equation is written as

$$
<u>\frac{\partial<u>}{\partial x}+<v>\frac{\partial<u>}{\partial y}=\frac{1}{\rho} \frac{\partial \tau}{\partial y}+U_{\infty} \frac{\mathrm{d} U_{\infty}}{\mathrm{d} x},
$$

with the total shear stress given by

$$
\tau=\rho \nu \frac{\partial<u>}{\partial y}-\rho<u^{\prime} v^{\prime}>,
$$

where $\rho<u^{\prime} v^{\prime}>$ is the Reynolds stress.

The shear stress at the wall, $\tau_{w}$, is an important quantity of the flow for experimental research and practical applications. At the wall, all convective terms are zero, so the shear stress is given by

$$
\tau_{w}(x)=\rho \nu\left(\frac{\partial<u>}{\partial y}\right)_{y=0}
$$

and the skin-friction coefficient is expressed as

$$
C_{f} \equiv \frac{\tau_{w}}{\frac{1}{2} \rho U_{\infty}^{2}}
$$

Near the wall, the viscosity $\nu$ and the wall shear stress $\tau_{w}$ are important parameters, thus viscous scales are defined. The friction velocity is given by

$$
u_{\tau} \equiv \sqrt{\frac{\tau_{w}}{\rho}},
$$

and the viscous length scale,

$$
\delta_{\nu} \equiv \nu \sqrt{\frac{\rho}{\tau_{w}}}=\frac{\nu}{u_{\tau}}
$$

The wall distance measured in viscous lengths, also called wall units, becomes $y^{+} \equiv y / \delta_{\nu}=u_{\tau} y / \nu$, and the non-dimensional mean stream-wise velocity $U^{+} \equiv<u>/ u_{\tau}=U / u_{\tau}$. In addition, the friction Reynolds number can be defined based on the viscous length scale, $R e_{\tau} \equiv \delta / \delta_{\nu}$.

Different regions and layers in the vicinity of the wall are defined with 
Table 2.1: Properties of wall regions and layers (adapted from [Pope, 2000]).

\begin{tabular}{|c|c|c|}
\hline Region & Location & Property \\
\hline inner layer & $y<0.1 \delta$ & $U$ depends on $y^{+}$and $u_{\tau}$ \\
\hline viscous wall region & $y<50 \delta_{\nu}$ & $\begin{array}{l}\text { significant contribution of viscosity to } \\
\text { the shear stress }\end{array}$ \\
\hline viscous sublayer & $y<5 \delta_{\nu}$ & $\begin{array}{l}\text { the Reynolds shear stress is negligible } \\
\text { compared to the viscous stress and } \\
\text { the linear relation } U^{+}=y^{+} \text {holds }\end{array}$ \\
\hline outer layer & $y>50 \delta_{\nu}$ & viscosity effects on $U$ are negligible \\
\hline overlap region & $50 \delta_{\nu}<y<0.1 \delta$ & joint effects of inner and outer layers \\
\hline log-law region & $30 \delta_{\nu}<y<0.3 \delta$ & $\begin{array}{l}\text { region controlled by the log-law } \\
U^{+}=(1 / \kappa) \ln \left(y^{+}\right)+C \text {, where } \kappa \text { and } \\
C \text { are the von Kármán empirical con- } \\
\text { stants }\end{array}$ \\
\hline buffer layer & $5 \delta_{\nu}<y<30 \delta_{\nu}$ & $\begin{array}{l}\text { region between the viscous sublayer } \\
\text { and the log-law region }\end{array}$ \\
\hline
\end{tabular}

respect to $y / \delta$ and $y^{+}$. In the viscous wall region $y^{+}<50$, there is a pronounced effect of the viscosity on the shear stress, whereas in the outer layer $y^{+}>50$ this effect is negligible, even though some interaction exists between the two regions. The viscous sublayer and the buffer region are both very thin compared with the local thickness of the boundary layer. Table 2.1 summarizes the limits and relevant properties of each region. It is important to mention that they are dependent of the Reynolds number and as the Reynolds number of the flow increases, the fraction of the near-wall region occupied by the viscous wall region decreases.

\subsection{Channel Flow}

A channel flow is a fluid flow confined by a conduit. This section describes relevant physical quantities of turbulent channel flows ([Pope, 2000]). Consider a developed flow through a rectangular duct of height $2 \delta$ and width $2 b$. The channel aspect ration is given by $A R \equiv \delta / b$. The bottom wall is located at $y=0$, the top wall at $y=2 \delta$ and the lateral walls at the span-wise positions $z=-b$ and $z=b$. The mean flow is predominant in the axial direction $x$ and is statistically one-dimensional in the centre planes of the channel, i.e., $(x, y)$ plane with $z=0$ and $(x, z)$ plane with $y=\delta$.

The Reynolds number based on the hydraulic diameter, $D \equiv$ 4 area/perimeter $=4 \delta b /(\delta+b)$, is used to characterize the flow ([Rohsenow and Hartnett, 1973])

$$
R e_{D} \equiv \frac{\bar{U} D}{\nu}
$$


where $\bar{U}$ is de bulk velocity defined as

$$
\bar{U} \equiv \frac{1}{\delta} \int_{0}^{\delta}<u>d y
$$

Near the entry of the duct $(x=0)$ there is a development region that depends on the type of the inlet and the flow Reynolds number. [Rohsenow and Hartnett, 1973] observed that, for an abrupt inlet and $R e_{D}=3000,40$ diameters are sufficient to develop the flow, while lower development distance is necessary for higher Reynolds numbers. The transition from the laminar to turbulent regime is also highly dependent on the configuration of the channel inlet. For an abrupt inlet, flows with $R e_{D}>2500$ are considered turbulent, while for a smooth inlet and channel aspect ratio of 1 the turbulent regime occurs only for $\operatorname{Re}_{D}>4300$ ([Rohsenow and Hartnett, 1973]).

Other important Reynolds number to characterize the flow can be defined based on the friction velocity and half-size of the channel.

$$
R e_{\tau} \equiv \frac{u_{\tau} \delta}{\nu}
$$

From the mean continuity equation, assuming $\langle w\rangle=0$, the independence of $\langle u\rangle$ in relation to $x$ (developed flow) and the boundary conditions $(<v>)_{y=0}=\left(\langle v>)_{y=2 \delta}=0,\langle v>\right.$ is equal to zero. So, the mean wallnormal momentum equation is simplified to

$$
-\frac{d<v^{\prime 2}>}{d y}-\rho \frac{\partial<p>}{\partial y}=0
$$

which, assuming that $\left(\left\langle v^{\prime 2}\right\rangle\right)_{y=0}=0$, integrates to

$$
<v^{\prime 2}>+\frac{<p>}{\rho}=\frac{p_{w}(x)}{\rho},
$$

where $p_{w}=<p>(x, 0,0)=<p>(x, 2 \delta, 0)$ is the mean pressure on the wall. This equation leads to a constant mean axial pressure gradient $\partial<p>/ \partial x=$ $d p_{w} / d x$.

The axial mean momentum equation can be written as

$$
\frac{d \tau}{d y}=\frac{d p_{w}}{d x}
$$

where the total shear stress is

$$
\tau=\rho \nu \frac{d<u>}{d y}-\rho<u^{\prime} v^{\prime}>
$$

Since $\tau$ depends only of $y$ and $p_{w}$ of $x$, the eq. (2.3.7) is equal to a constant. Hence, the pressure gradient can be written in terms of the wall 
shear stress $\tau_{w} \equiv \tau(0)=-\tau(2 \delta)$.

$$
-\frac{d p_{w}}{d x}=\frac{\tau_{w}}{\delta}
$$

In a channel flow, the flow is driven by the drop in pressure between the inlet and outlet of the conduit. The constant and negative mean pressure gradient is balanced by the shear stress gradient.

The skin-friction coefficient is defined based on the wall shear stress

$$
C_{f} \equiv \frac{\tau_{w}}{\frac{1}{2} \rho \bar{U}^{2}}
$$

For fully-developed channel flows, the skin-friction coefficient can be estimated by empiric correlations for pipe flow. [Hartnett et al., 1962] concluded that, for $6 \times 10^{3}<R e_{D}<5 \times 10^{5}$, the correlations for round pipe represent faithfully the flow inside channels with any aspect ratio. The skin-friction coefficient can be obtained by Karman-Nikuradse equation ([Rohsenow and Hartnett, 1973]).

$$
\frac{1}{\sqrt{4 C_{f}}}=-0.8+0.87 \ln \left(R e_{D} \sqrt{4 C_{f}}\right)
$$

Other possibility is the Colebrook-White equation for the skin-friction coefficient ([Rohsenow and Hartnett, 1973]) as follows.

$$
\frac{1}{\sqrt{4 C_{f}}}=-2 \log _{10}\left(\frac{2.51}{R e_{D} \sqrt{4 C_{f}}}\right)
$$

The Karman-Nikuradse and Colebrook-White equations, for $5 \times 10^{3}<R e_{D}<3 \times 10^{4}$, can be simplified to the Blasius' equation ([Rohsenow and Hartnett, 1973]) given by

$$
C_{f}=0.079 R e_{D}^{-1 / 4}
$$

The turbulent velocity profile inside smooth pipes can be represented by a empiric power-law ([Fox et al., 1998]).

$$
\frac{U}{U_{\max }}=\left[\frac{y}{\delta}\right]^{1 / n}
$$

where $U_{\max }$ is the maximum mean velocity inside the duct and the exponent $n$ varies with $R e_{D}$.

Analogously to boundary-layer flows, close to the wall, the viscosity and wall shear are important parameters used to define the friction velocity, $u_{\tau} \equiv$ $\sqrt{\tau_{w} / \rho}$, and the viscous length scale, $\delta_{\nu} \equiv \nu \sqrt{\rho / \tau_{w}}$. These parameters are employed to the non-dimensionalisation of velocity and distance, respectively. The behaviour of the flow in the near-wall region for channel flows is similar to boundary-layer flows and the same regions and layers described in the Table 2.1 are valid. 


\subsection{Turbulent Structures}

With the sophistication of measurement techniques and development of computers used in numerical simulations, studies on near-wall turbulence consistently indicate the existence of so-called "coherent structures" ([Robinson, 1991a, Robinson, 1991b]). These coherent structures were defined by [Robinson, 1991a] as regions of the flow over which a fundamental flow variable exhibits significant correlation with itself or another variable over a range of space or time larger than the smallest local scales of the flow. These structures can be classified as low- and high-speed streaks ([Kline et al., 1967]), ejections and sweeps ([Corino and Brodkey, 1969]), vortices ([Robinson, 1991b]), among others. Along the text in the present work, the expression turbulent structures can be used with the same meaning as coherent structures.

\subsubsection{Low- and High-Speed Regions}

Streaks were firstly investigated by [Kline et al., 1967] in boundary layers subjected to different pressure gradients using hydrogen bubble wire visualization technique. They observed that the low speed streaks exist in all cases and show a remarkable degree of persistence and regularity as displayed in figure 2.1. Near the wall, the instantaneous streamwise velocity consists of alternating regions of high and low speed fluid along the spanwise direction. The mean spanwise spacing between low-speed streaks for low Reynolds number seems to be about $\triangle z=100 \pm 20$ wall units at $y^{+} \leq 10$ and increases for $y^{+}>10$ ([Kline et al., 1967, Achia and Thompson, 1977, Oldaker and Tiederman, 1977, Blackwelder and Eckelmann, 1979, Nakagawa and Nezu, 1981, Smith and Metzler, 1983]). This mean spanwise spacing of low-speed streaks also increases with Reynolds number ([Gupta et al., 1971, Kähler, 2004, Lagraa et al., 2004, Carlier and Stanislas, 2005]).

The streak structures displays a length scale generally of $\Delta x^{+}>1000$ ([Oldaker and Tiederman, 1977, Blackwelder and Eckelmann, 1979, Carlier and Stanislas, 2005]) and a width of $20<\triangle z^{+}<60$ ([Robinson, 1991b, Carlier and Stanislas, 2005]). For $y^{+}>40$, the streaky structure of the streamwise velocity is barely noticeable ([Smith and Metzler, 1983]), although long streamwise velocity fluctuation in the outer region extending up to $8 D$ was found in pipe flows ([Kim and Adrian, 1999, Guala et al., 2006]). The streaks exhibit a sinuous character with an extend up to $20 \delta$ ([Hutchins and Marusic, 2007]), commonly branching and reconnecting ([Kline et al., 1967]). [Johansson et al., 1991] showed that the development of asymmetry in the spanwise direction is important for the evolution of near-wall structures and that high-speed streaks 


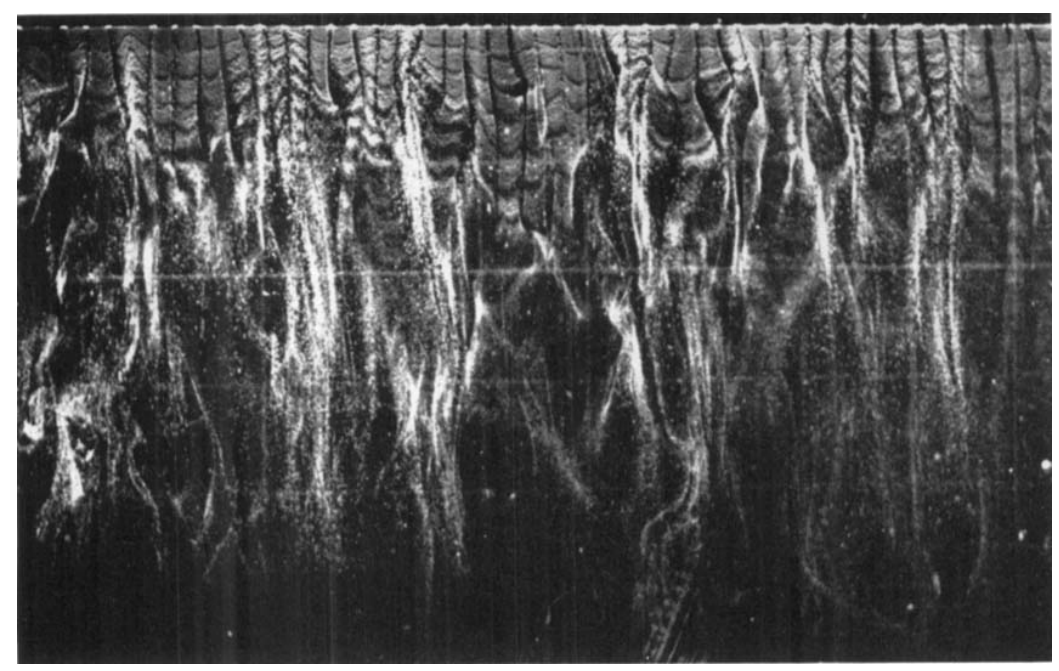

Figure 2.1: Structure of the flat plate turbulent boundary layer as visualized with the hydrogen bubble technique of [Kline et al., 1967]. The wire is located parallel to the plate and normal to the direction at of flow at $y^{+}=9$. The flow is from top to bottom of the picture. The bubbles were collected into long streaks.

are surrounded by spanwise diverging fluid.

[Talmon et al., 1986] found that high-speed streaks are related to regions of high values of Reynolds stresses. The studies of [Robinson, 1991a] associated the high-speed streaks with an exchange of wall-normal velocity to streamwise and spanwise velocity.

The origin of streaks can be explained by two models: as a consequence of counter-rotating pairs of streamwise vortices ([Blackwelder and Eckelmann, 1979]) or as a combined action of lift-up, shear and diffusion of the fluid ([Landahl, 1990]).

\subsubsection{Ejections and Sweeps}

The study of ejections and sweeps was carried out by [Corino and Brodkey, 1969] in a fully developed turbulent pipe flow that was photographed by a high-speed camera moving with the flow. Ejections are defined as regions of an abrupt outward motion of fluid with low streamwise velocity, while sweeps are inward motion of fluid with high stream-wise velocity. According [Corino and Brodkey, 1969], the process of ejection firstly starts with a decelerated region with a streamwise velocity equal to $50 \%$ of the local mean velocity followed by an accelerated mass of fluid from the upstream region moving wall-ward at angles $0-15^{\circ}$ that gradually penetrates the decelerated flow. Due to the acceleration of the fluid, an ejection of low speed fluid away from the wall is observed. As a consequence, a sweep event given by a wall-ward flow occurs to replace the fluid mass pumped outward during ejections (continuity). 
These deterministic events occurring randomly in space and time are the main responsible to the process of Reynolds stress production ([Corino and Brodkey, 1969, Grass, 1971, Wallace et al., 1972, Lu and Willmarth, 1973, Blackwelder and Kaplan, 1976, Talmon et al., 1986, Kim et al., 1987, Carlier and Stanislas, 2005, Lin, 2006, Jiménez and Lozano-Durán, 2016]). The contribution of ejection events in the Reynolds stress production was reported to be more relevant than sweep events ([Corino and Brodkey, 1969, Wallace et al., 1972, Dekou et al., 2016]).

Some theories were conjectured to explain the origin of ejections and sweeps. [Delo et al., 2004] evidenced that the ejection events are related with the lift up of low speed streaks by using a volumetric imaging technique. From direct numeric simulations, [Álamo et al., 2006] observed that, on average, wallnormal ejections were surrounded by two inclined counter-rotating vortices.

\subsubsection{Vortices}

A vortical structure is defined for the present purposes as an instantaneous vortex, generally forming a complex three-dimensional shape ([Robinson, 1991b]). There is a consensus that vortical structures play a central role in the production of boundary-layer turbulence. Any vortex with an orientation other than wall-normal has the potential to function as a "pump" which transports mass and momentum across the mean velocity gradient ([Robinson, 1991b]). A significant portion of the boundary-layer structure literature is devoted to detection and characterization of vortical elements and structures as hairpins, canes, horseshoes, streamwise vortices, transverse vortices, rings, among others.

[Theodorsen, 1952] suggested the existence of horseshoe vortices in wall turbulence. Instabilities of the instantaneous velocity profiles evolving into transverse vortices that are stretched by the shear into intense hairpin vortices ([Theodorsen, 1952, Klebanoff et al., 1962]). These hairpin vortices were supposed to be tilted at about $45^{\circ}$ downstream ([Theodorsen, 1952, Robinson, 1991a, Adrian, 1991, Robinson, 1991a, Ganapathisubramani et al., 2006]), due to the stretching of the mean velocity gradient and to the mechanism of self-induction which lifts them up. Hairpin (or horseshoe) vortices are the major momentum-transporting structures in shear turbulence ([Theodorsen, 1952]). [Townsend, 1980] found that the legs of horseshoe vortices would remain attached to the wall during their development. The mean spacing between these legs is about 100 wall units, but the aspect ratio of the horseshoe vortices is Reynolds number dependent ([Head and Bandyopadhyay, 1981]). These hairpin vortices generally appear more asymmetric, having a cane-like shape 


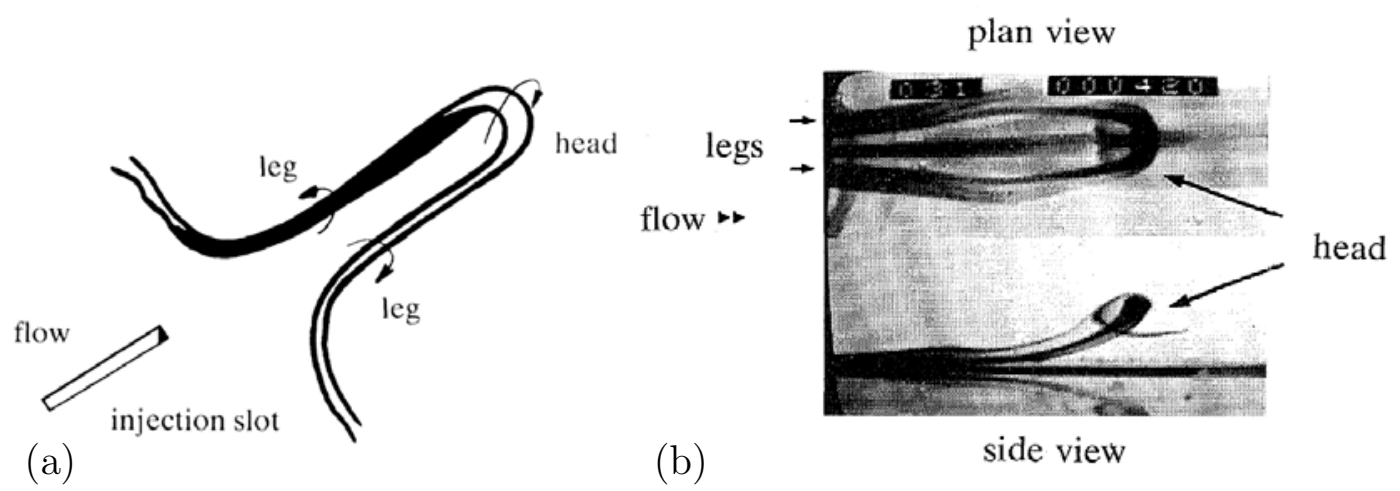

Figure 2.2: (a) Isometric schematic view of single hairpin vortex and (b) dualview picture of dye-market single hairpin vortex from the turbulent boundarylayer experiments of [Smith et al., 1991]

(with two legs of different lengths) than symmetric ([Robinson, 1991a, Jeong et al., 1997, Carlier and Stanislas, 2005, Herpin et al., 2013]) and usually travel in groups at a uniform streamwise velocity ([Head and Bandyopadhyay, 1981, Smith et al., 1991, Zhou et al., 1999, Adrian et al., 2000b, Marusic, 2001, Tomkins and Adrian, 2003, Delo et al., 2004]).

Figure 2.2 shows a schematic sketch of a single hairpin vortex and the visualization of such vortex in a "kernel" experiment of [Smith et al., 1991] in a boundary layer. Single hairpin vortices are generated by surface injection.

[Bakewell and Lumley, 1967] found pairs of counter rotation vortices near the wall along the streamwise direction that was confirmed by other researches ([Kim et al., 1971, Grass, 1971, Blackwelder and Eckelmann, 1979, Smith and Metzler, 1983, Aubry et al., 1988, Sheng et al., 2008]). [Guezennec et al., 1989] showed that the streamwise vortices do not necessarily appear in pairs and rarely have the same amplitude. These streamwise vortices have diameters ranging from 10 to 40 wall units ([Gupta et al., 1971, Blackwelder and Eckelmann, 1979, Kim et al., 1987, Carlier and Stanislas, 2005, Herpin et al., 2010]), are centred at 10 to 50 wall units from the wall and are separated by 50 to 100 wall units in the transverse direction ([Gupta et al., 1971, Blackwelder and Eckelmann, 1979, Kim et al., 1987]). The radius of streamwise-oriented vortical structures in the logarithmic region were found to be universal both in Reynolds number and in wall-normal distance when scaled with the local Kolmogorov scales $\mathrm{u}_{\eta}$ and $\eta$ ([Tanahashi et al., 2004, Álamo et al., 2006, Stanislas et al., 2008]) with most probable diameter of $10 \eta$ and most probable vorticity around $1.6 u_{\eta} \eta^{-1}$ ([Stanislas et al., 2008]). These quasistreamwise vortices slightly tilt upwards from the wall $\left(3\right.$ to $\left.7^{\circ}\right)$ in the nearwall region and make angles of approximately $45^{\circ}$ in the outer region ([Carlier and Stanislas, 2005, Ganapathisubramani et al., 2006, Gao et al., 2011]), which 


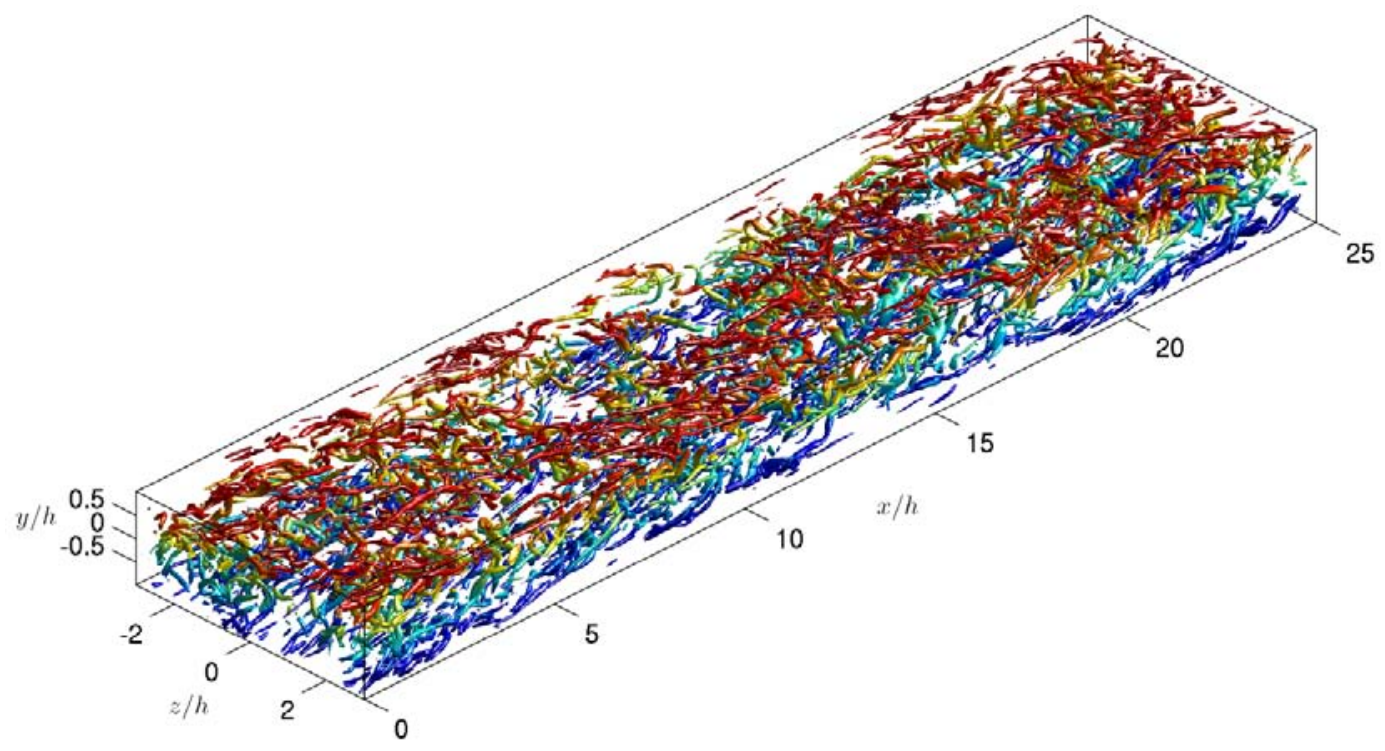

Figure 2.3: Coherent vortices extracted from the direct numerical simulation of a flow inside a duct with $R e_{\tau}=180$ ([Vinuesa et al., 2014]). Colour code by wall-normal direction: blue refers to vortices closer to the lower wall, while red to the ones closer to the upper wall. Flow is from lower-left corner to upper-right. $h$ is the half channel height.

corresponds to the direction of maximum vorticity production due to stretching by the mean gradient ([Robinson, 1991b]). These vortices contain the largest amount of energy of the flow ([Bakewell and Lumley, 1967]). [Grass, 1971] observed quasi-streamwise vortices, generally during near-wall ejection and sweep events.

Although it was mentioned that there are preferred angles for the main turbulent structures, the instantaneous flow displays a variety of vortex inclinations that can be found in the visualizations observed in the literature [Scarano and Poelma, 2009, Atkinson et al., 2011, Schröder et al., 2011, Foucaut et al., 2011a, Jodai et al., 2014, Vinuesa et al., 2014]. Figure 2.3 shows coherent vortices, defined using the Q-criterion ([Hunt et al., 1988]), from direct numerical simulation of a flow inside a duct of aspect ration of 3 with $R e_{\tau}=180$ ([Vinuesa et al., 2014]).

The origins of streamwise vortices can be classified into five groups, namely: hairpin vortex related mechanism ([Adrian et al., 2000b]), streamwise vorticity sheet roll up ([Bernard et al., 1993]), instability of curvature and shear stress near the wall ([Brown and Thomas, 1977]), oblique mode theory ([Schoppa and Hussain, 2002]) and streak instability ([Robinson, 1991a]). 


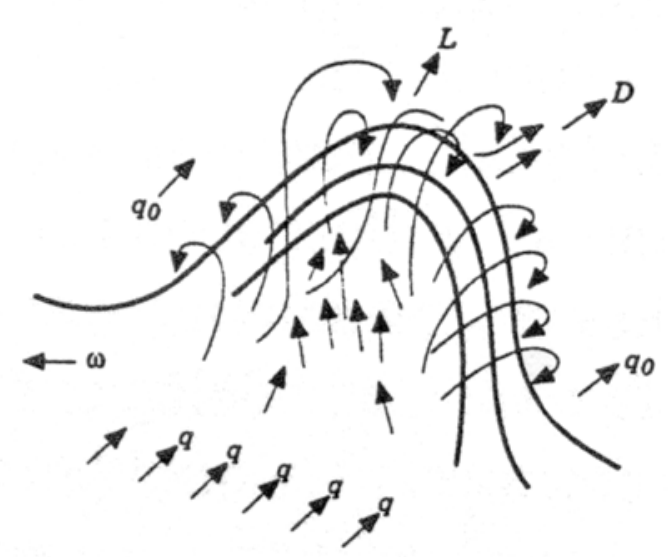

Figure 2.4: Illustration of horseshoe vortex ([Theodorsen, 1952]).

\subsubsection{Turbulent Structure Organization and Interaction}

For 60 years, many researches have been attempting to understand the generation, the interaction mechanism and the contribution to the production and dissipation of turbulent structures. The first detailed description of the organization was proposed by [Theodorsen, 1952]. In this model, illustrated in figure 2.4, the instability of the streamwise instantaneous velocity profiles is responsible to create spanwise vortices (Kelvin-Helmholtz instability) that, under the action of the mean velocity gradient and instabilities of the flow, evolve into deformed vortex tubes as streamwise or hairpin vortices. Both vortical structures explain the formation of the low-speed streaks close to the wall, which is responsible for the mass transfer away from the wall (ejections). This outward mass transfer is compensated by sweep motions toward the wall. The creation of new vortical structures is attributed to the instability of the instantaneous velocity profile due to the streaks and to an induction mechanism by the already existing vortices.

Later, [Hinze, 1975] proposed a cyclic process that starts with a horseshoe-shaped vortex ([Theodorsen, 1952]), which is formed at the wall due to a large-scale perturbation (see figure 2.5). This initial vortex is deformed by the flow into an elongated U-shaped loop. Due to the self-induction process, the tip of the loop moves away from the wall and is disturbed by the velocity gradient. Between the vortex legs near the wall, a local deceleration of the fluid appears (low-speed streaks). At distances $x^{+}=5$ to 30, an intense horizontal shear-layer is formed. As a consequence of this local instability a breakdown of the flow surrounding the original tip of the vortex produces a turbulence burst (sometimes called ejection). The pressure waves associated with the burst are propagated through the entire boundary layer. The fluid with high turbulence intensity produced during the burst is convected down- 


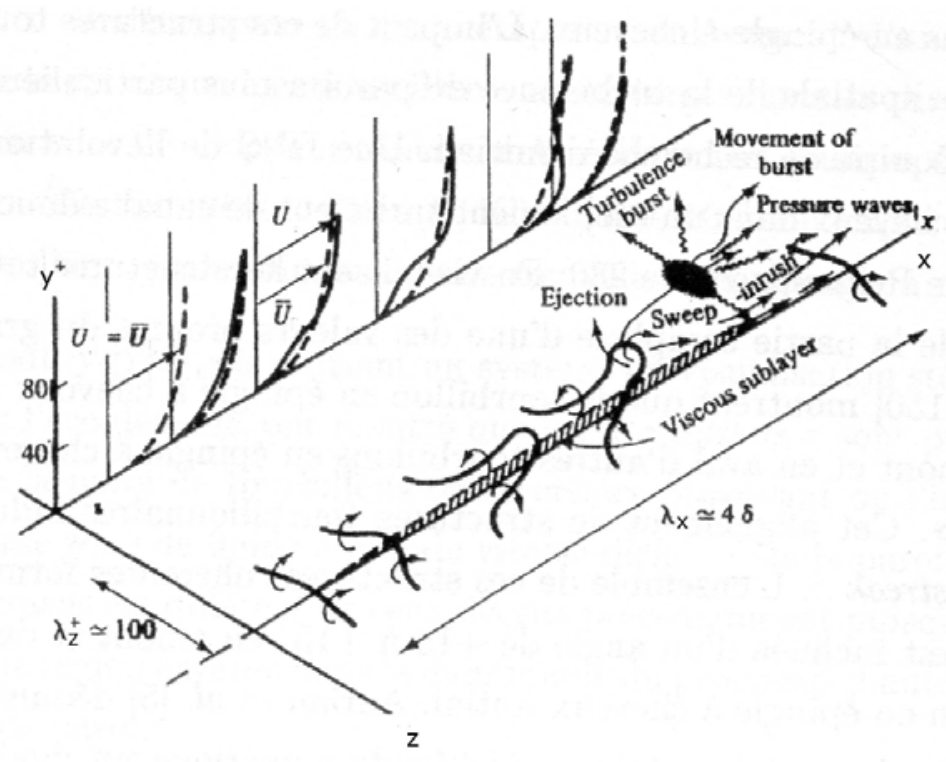

Figure 2.5: Organization model of [Hinze, 1975] in near-wall turbulence.

stream and moves farther away from the wall. As a result, high-momentum fluid enters from upstream (sweep).

[Smith and Walker, 1997] found that hairpin vortices in a shear flow are able to interact with other three-dimensional vortices to yield larger-scale flow structures, and regenerate new vortices through an interaction with the viscous wall layer. The evolution of the hairpin vortex near the wall can induce low speed streaks. If the original vortex is sufficiently strong, a burst may occur as a result of the sustained action of the hairpin vortex in the wall layer. The process is reflected as a local viscous-inviscid interaction that initiates close to the streak, appears to destabilize it, and culminates in the ejection of a portion of the streak and secondary vorticity into the out region. If original vortex is relatively weak, it can cause a streak to form. In this case, a streak will either diffuse or be over-run by subsequent impinging hairpin vortices. The subsequent hairpin vortices can either refocus the streak, causing its growth by the combination with other adjacent streaks, or buffet the original streak. The subsequent viscous inviscid interaction can result in the formation of one or more secondary hairpin vortices. When low speed fluid is ejected outward near a streak, an inflow of high speed fluid (sweep) will occur to recover the mean velocity profile which will appear and be detected as a local acceleration of the flow. The processes occurring in the near-wall are cyclical. Wall-region vortices interact with and cause ejections of wall-region fluid, which subsequently roll up to form new vortices by a viscous-inviscid interaction with the high speed, outer-region fluid. This process defines a continuing cycle which contains both the elements which sustain turbulence (three dimensional vortices) and the 


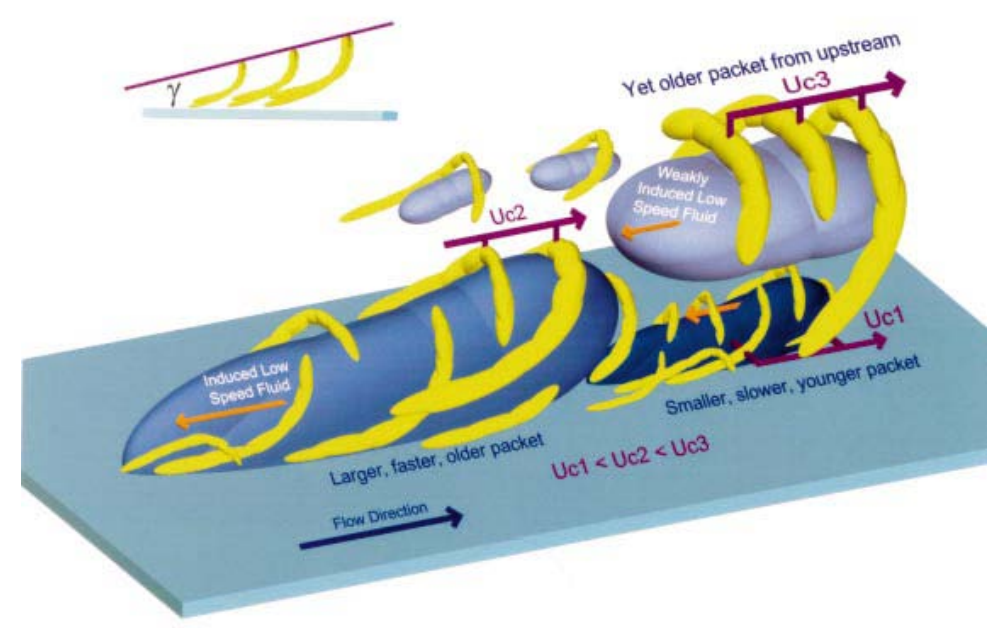

Figure 2.6: Organization model of [Adrian et al., 2000b] in near wall turbulence, $U_{c 1}, U_{c 2}$ and $U_{c 3}$ are the convection velocities of each hairpin packet respectively.

process for regeneration (viscous-inviscid interactions).

The model based on hairpin vortical structures can explain many observed flow events. [Zhou et al., 1999] showed the ability of a single hairpin structure to generate secondary hairpins in a direct numerical simulation (DNS). The hairpin vortices remain grouped, inducing an elongated area of low-momentum fluid. The organization of these turbulent structures in packets was studied experimentally by [Adrian et al., 2000b] using particle image velocimetry (PIV) in a near-wall region of a turbulent boundary layer at Reynolds number of $930<R e_{\theta}<6845$. The hairpins frequently appeared in groups with a small dispersion in their convection velocity, aligned in the streamwise direction and inclined of $12^{\circ}$ from the wall. The packets had a streamwise length of $1.3 \delta$ and were spaced several hundreds of viscous length scales. [Adrian et al., 2000b] suggested the conceptual model displayed in figure 2.6. In this model, hairpin packets originate at the wall from a disturbance. The primary hairpin is formed, stretched and intensified by the difference between the streamwise velocity at its legs and its head. The hairpin grows continuously in time, changing from a hairpin-shape to an omega-shape, and generates a new upstream hairpin by inducing a strong, three-dimensional ejection event that interacts with high-speed fluid behind the primary hairpin. The secondary hairpin increases in size with time and can create a tertiary hairpin.

Evidences of hairpin-like packets as an important feature of wall turbulence were found by other researchers using different experimental techniques ([Christensen and Adrian, 2001, Tomkins and Adrian, 2003, Ganapathisubramani et al., 2003, Hutchins and Marusic, 2007, Schröder 


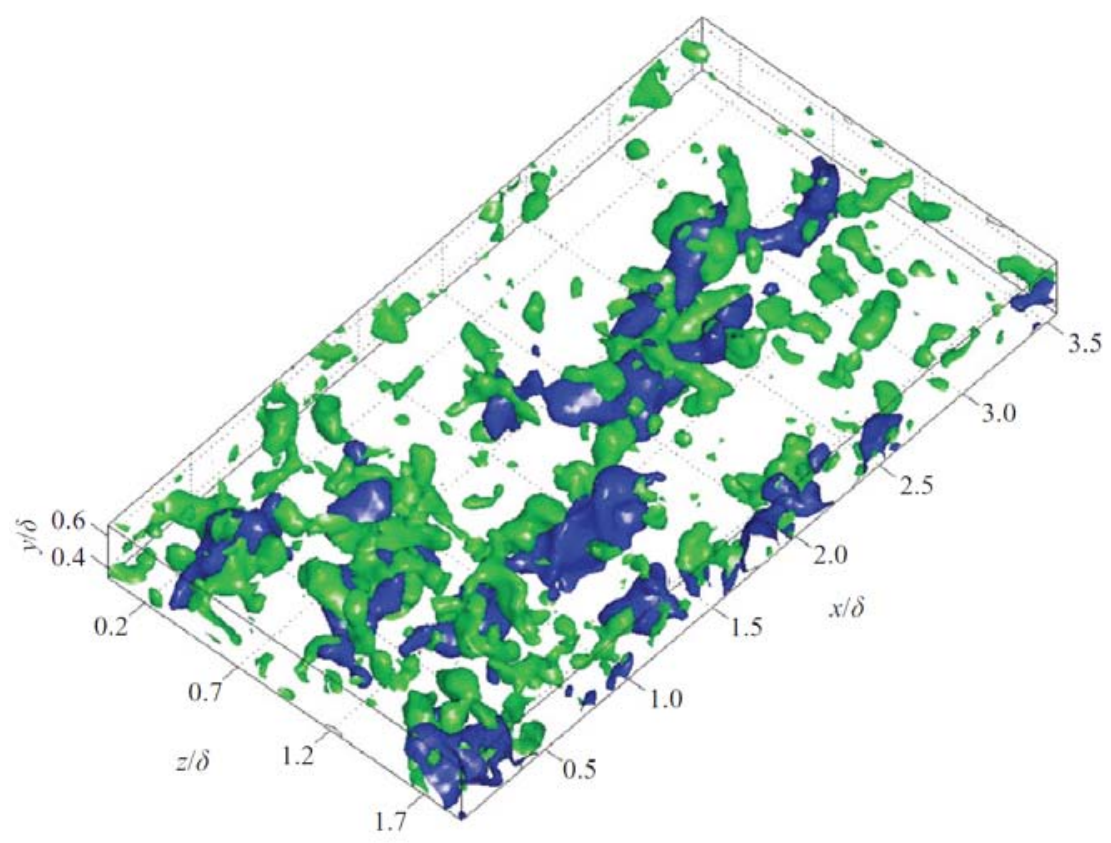

Figure 2.7: Instantaneous vortex distribution detected by the $\mathrm{Q}$ criterion (green) and low-speed zones of $u<0.89 U$ (blue) for $0.34<y / \delta<0.67$ ([Elsinga et al., 2010]).

et al., 2008b, Elsinga et al., 2010, Marusic et al., 2010, Dennis and Nickels, 2011a, Schröder et al., 2011, Gao et al., 2013, Jodai et al., 2014]).

As an example of these works, figure 2.7 shows an instantaneous tomo-PIV result from a supersonic turbulent boundary layer at Reynolds number based on momentum thickness of $R e_{\tau}=34000$ from [Elsinga et al., 2010]. The plot revels that most vortical structures are concentrated near the lowspeed zones in agreement to [Adrian et al., 2000b].

Despite the fact that a large amount of work on coherent structures has already been conducted, a number of aspects of the organization of nearwall turbulence remain unclear and sometimes even controversial in the light of these recent results ([Pope, 2000, del Álamo et al., 2006, Stanislas et al., 2008, Foucaut et al., 2011b]). The qualitative analysis of a few instantaneous velocity fields certainly provides useful scenarios for turbulence organization. However, numerical or experimental statistical results on coherent structures are required to assess how representative these models are. 


\section{3 \\ Tomographic PIV}

This chapter covers the last tomo-PIV developments since the introduction of this optical measurement technique. It describes the working principle and highlights some relevant parameters of the tomographic PIV. The last part of the chapter discusses a methodology, based on indicators, that should be applied along the phases of the tomo-PIV processing to estimate the quality of the data.

\subsection{Tomographic PIV Developments}

Since the introduction of tomographic PIV by [Elsinga et al., 2005b, Elsinga et al., 2005a] the research community has become interested in this three-dimensional, three-component (3D-3C), and sometimes still timeresolved, non-intrusive technique. In recent years, the technique demonstrated to be suitable for studying three-dimensional unsteady flows, as summarized in recent reviews of [Scarano, 2013] and [Westerweel et al., 2013].

Tomographic PIV (shortly tomo-PIV) is based on the reconstruction of particle distribution inside an illuminated volume of a seeded flow. The light scattered from the particles is recorded by two or more different camera views. The instantaneous flow is estimated by the particle displacement between two light pulses computed by a 3D cross-correlation of a pair of reconstructed volumes calculated from these views.

The reconstruction of particles from a limited number of views is an under-determined system solved by algebraic techniques ([Herman and Lent, 1976]), which are very time consuming ([Worth and Nickels, 2008, Atkinson and Soria, 2009, Scarano and Poelma, 2009, Discetti et al., 2013, Thomas et al., 2014]). The Algebraic Reconstruction Technique (ART) ([Gordon et al., 1970]) and the Multiplicative Algebraic Reconstruction Technique (MART) ([Herman and Lent, 1976]) for tomo-PIV were introduced by [Elsinga et al., 2006]. At that time, the MART algorithm demonstrated its potential and became the most popular reconstruction technique until today.

One problem associated with the volume reconstruction with limited 


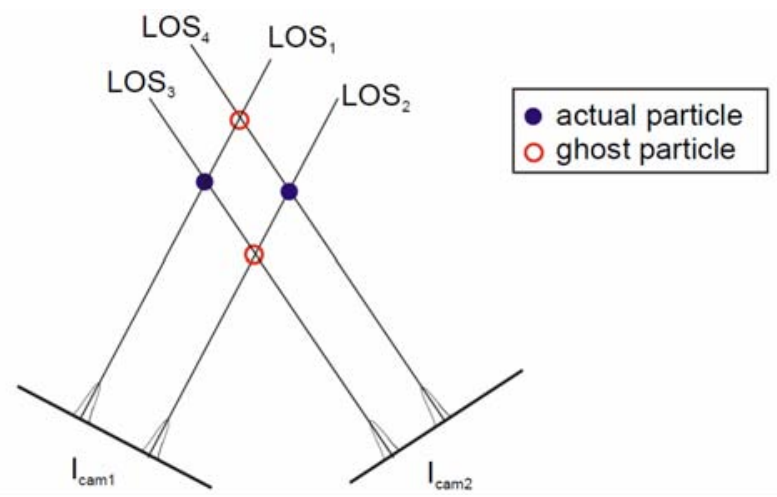

Figure 3.1: Formation of ghost particles in a 2-camera tomo-PIV setup. ([Elsinga, 2008]).

views is the ambiguity that results in a larger number of particles to be formed, as compared to those actually present in the illuminated volume. Such extra particles were referred to as "ghost particles" by [Maas et al., 1993]. The number of ghost particles produced by MART can be comparable, and often larger, than the number of actual particles, and it depends mainly on three experimental parameters, namely, the number of simultaneous views, the seeding density and the thickness of the illuminated volume ([Maas et al., 1993]). On the other hand, many ghost particles do not produce a coherent pattern in the correlation map, which makes the cross-correlation analysis of tomo-PIV significantly less affected by ghost particles ([Elsinga et al., 2006, Elsinga, 2008]). Nevertheless, the bias error in velocity measurements by the coherent motion of ghost particles under some circumstances leads to a systematic underestimation of the magnitude of the particle displacement gradient ([Elsinga et al., 2011, Elsinga and Tokgoz, 2014]) and increases random velocity errors ([Novara et al., 2010, de Silva et al., 2013]).

Figure 3.1 illustrates the formation of false or ghost particles in a 2camera system. In this example, reproduced from [Elsinga, 2008], the measurement volume contains two particles, which are seen by camera 1 along the lines-of-sight (LOS) 1 and 2 and by camera 2 along the lines 3 and 4 . In the reconstruction process these recorded particle images and corresponding LOS 1 to 4 are used to determine the particle distribution in the measurement volume. Particles are formed where the lines-of-sight corresponding to a particle image in each camera intersect.

The performance and accuracy of tomo-PIV is highly dependent on the reconstruction techniques employed. For this reason, much effort is being made to optimize these algorithms. [Petra et al., 2007] analysed tomo-PIV from a mathematical point of view. The reconstruction techniques ART, a modification of this algorithm based on constraining strategies ([Koltracht and 
Lancaster, 1990]) called ART+pos and the Sequential Linearization Algorithm (SLA) ([Mangasarian, 1999]) were tested. The SLA reconstructions, which have a computational cost considerably higher than the others, showed more distinct particles and less amount of ghost particles, followed by ART+pos and MART. Experimental investigations are nevertheless necessary to further verify these predictions ([Scarano, 2013]).

Alternative techniques, as the Adaptive Algebraic Reconstruction Technique (AART) ([Lu and Yin, 2004]), the Simultaneous Iterative Reconstruction technique (SIRT) ([Herman and Lent, 1976]) and the Improved Iterative Algorithm for Sparse Object Reconstruction (IIASOR) ([Li et al., 2004]) were studied by [Atkinson and Soria, 2007]. In that work, MART has shown to produce the best results.

[Worth and Nickels, 2008] revisited the uniform initial volume intensity distribution, which was adopted by [Elsinga et al., 2006], and proposed a Multiplied First Guess (MFG) to significantly accelerate the solution convergence of reconstruction. [Atkinson and Soria, 2009] also proposed an equivalent fast, one-pass multiplication along the line of sight (MLOS). In a different approach, [Putze and Maas, 2008] suggested a minimum line-of-sight intensity as the initial guess (called MinLOS). Later, [Thomas et al., 2010] compared different initialization approaches and concluded that, for lower particle densities, the use of MinLOS is more suitable. The use of volume reconstruction employing only multiplicative line-of-sight or minimum line-of-sight techniques was investigated by [Michaelis et al., 2010]. The results revealed a good compromise of computational time and accuracy only for very low seeding densities.

[Atkinson and Soria, 2009] presented a combination of a multiplicative line-of-sight (MLOS or MFS) with iterations of the Simultaneous Algebraic Reconstruction Technique (SART) ([Andersen and Kak, 1984]), which was referred to as MLOS-SART, and a simultaneous implementation of the Simultaneous Multiplicative Algebraic Reconstruction Technique (SMART) ([Mishra et al., 1999]), named MLOS-SMART. This MLOS-SMART achieved better performance than MART. This fact was after confirmed by [Bilsky et al., 2011] and [Thomas et al., 2014], albeit the latter authors detected a slight quality loss.

[Byrne, 2009] discussed several block-iterative optimization methods, among them the Block-Iterative MART (BIMART) that was later investigated by [Thomas et al., 2014]. This block reconstruction method was 2 times faster than MART with only a slight accuracy penalty.

[Gesemann et al., 2010] introduced two variations of the non-monotone Spectral Projected Gradient method (SPG) ([Birgin et al., 2000]), designated 
S-SPG and L1S-SPG. The L1S-SPG implementation required about twice as much working memory as compared to SMART, but gives better results after a few iterations in cases of high particle density or noisy images. The work suggested a combination of SMART for the initial iterations followed by L1S-SPG iterations. Also in this case, further investigation is needed ([Scarano, 2013]).

Exploring the mentioned effect that most ghost particles do not correlate, [Novara et al., 2010] introduced the Motion Tracking Enhancement (MTE) technique by combining the reconstruction procedure and displacement estimation. The technique needs significant more computational effort than standard 5-iterations MART, but has the potential to achieve more accurate results than the usual tomo-PIV, especially at higher seeding densities ([Michaelis et al., 2010, Novara and Scarano, 2012, Novara, 2013]). Further, [Elsinga and Tokgoz, 2014 ] proposed the ultimate-MTE-MART that removes ghosts in the reconstruction volume based on both the intensity and the particle-track length. The improved method increased the accuracy only for moderate seeding densities. Recently, [Lynch and Scarano, 2015] implemented the Sequential MTE-MART (SMTE-MART) that significantly reduces the computational time for reconstruction. Moreover, the method yielded superior reconstruction quality and higher velocity field measurement accuracy when compared with both MART and MTE-MART in synthetic and experimental tests.

In order to reduce the computational cost, [Bilsky et al., 2012] proposed the use of the Maximum Entropy Reconstruction Technique (MENT) ([Minerbo, 1979]). MENT showed to be faster than SMART, but less accurate.

[Discetti and Astarita, 2012] implemented a fast multi-resolution (MR) reconstruction approach, consisting in the initial reduction in the dimension of the reconstruction problem by means of an image compression. This procedure performed the reconstruction noticeably faster than standard MART, besides it allowed a reduction of the memory needed to store the weighting matrix while preserving the quality.

[Thomas et al., 2010, Thomas et al., 2014] made a thorough study on the impact on volume reconstruction of different parameters such as image preprocessing, volume discretization, weighting matrix, algorithm initialization, camera angles, volume thickness and particle density. In this synthetic and experimental work, the standard weighting matrix, computed by the intersection of a sphere and a cylinder [Lamarche and Leroy, 1990], was revisited and a new implementation computed by a bilinear interpolation of the voxel intensity at the pixel back-propagated position was suggested.

In cases of particle image aberrations, instead of assuming a spatially 
homogeneous weighting function to relate voxels to image space, a variable optical transfer function (OTF) was introduced by [Schanz et al., 2013a]. In their study, the OTF improved the reconstruction quality, the accuracy of particle peak position and the displacement vector field, as compared to the standard approach, B-spline and bilinear functions in a synthetic study.

[Discetti et al., 2013] proposed the Spatial Filtering Improved Tomography (SFIT), in which the reconstructed intensity distributions are filtered by means of a Gaussian window to reduce the error due to voxel-based discretization. The effects of anisotropic and isotropic spatial filtering were investigated. The authors concluded that the intensity of the ghost particles were distributed on the actual particles, noticeably enhancing the reconstruction quality.

The Simulacrum Matching-based Reconstruction Enhancement (SMRE) technique, which utilizes the characteristic shape and size of actual particles to remove ghost particles in the reconstructed intensity field, was proposed by [de Silva et al., 2013]. Their work indicated that most often ghost particles were weaker than true particles (as also noticed by [Elsinga et al., 2006, Elsinga and Tokgoz, 2014]) and irregularly shaped. For [Elsinga and Tokgoz, 2014], the ability of the original gamma criterion ([de Silva et al., 2013]) to remove some ghost particles should be ascribed largely to intensity differences rather than size differences.

Recently the "Shake the Box" (STB) Particle Tracking Velocimetry (PTV) ([Schanz et al., 2013b, Schanz et al., 2014]), which incorporates the temporal information into the process of particle position determination, demonstrated its high accuracy for densely seeded flows. This Lagrangian approach, based on the image matching schemes introduced in the Iterative Particle Reconstruction (IPR) ([Wieneke, 2013]), showed to be faster than the standard reconstruction method and to practically solve the ghost-particle problem by removing small particle tracks (similar procedure adopted by [Schröder et al., 2011]). Figure 3.2 shows vortices, detected by a swirl strength criteria criterion ([Zhou et al., 1999]), and vectors of an instantaneous tomo-PIV field from the water-tunnel experiment of [Schanz et al., 2013b]. 


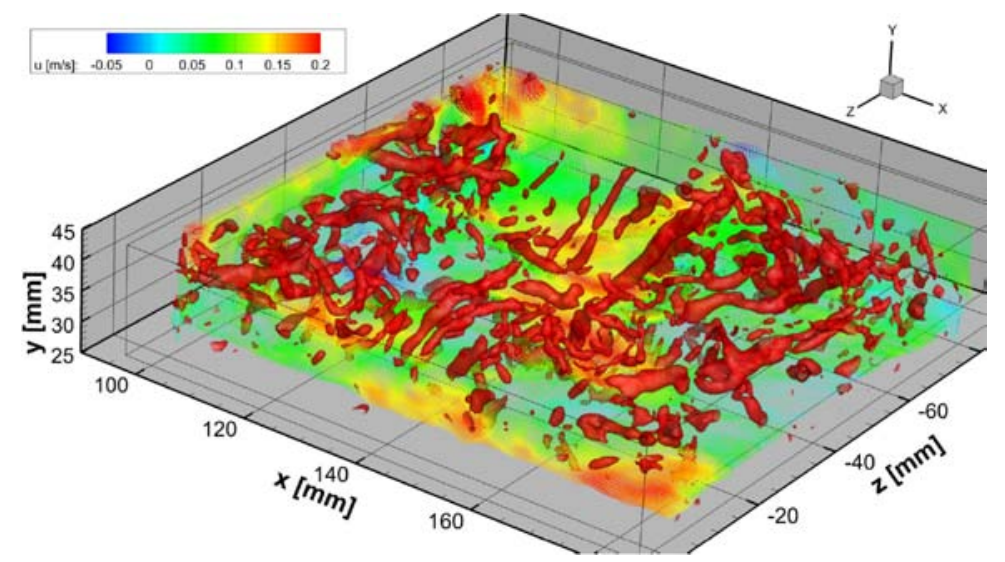

Figure 3.2: Instantaneous water-tunnel tomo-PIV result at $R e=8000$ ([Schanz et al., 2013b]). Isosurfaces of 3D swirl strength $\lambda_{c i}$ with vector slices color-coded by streamwise velocity $u$.

\subsection{Working Principle}

The essence of Tomo-PIV, which is sketched in figure 3.3, is summarized as follows. Each item is discussed in the next sections.

1. The camera system, two or more digital cameras differently oriented relative to the measurement volume, is calibrated. Self-calibration follows the initial calibration to correct errors.

2. The flow is seeded with particle tracers.

3. The volumetric measurement region is twice illuminated by a light pulse with a controlled time delay.

4. Scattered light of particles is recorded by the cameras.

5. The actual positions of tracers in the measurement volume are determined by tomographic reconstruction methods using obtained particle images and information about spatial calibration of the cameras.

6. The velocity field is estimated by the particle shifts between light pulses, which are calculated cross-correlating a pair of reconstructed volumes containing the particle distributions. 


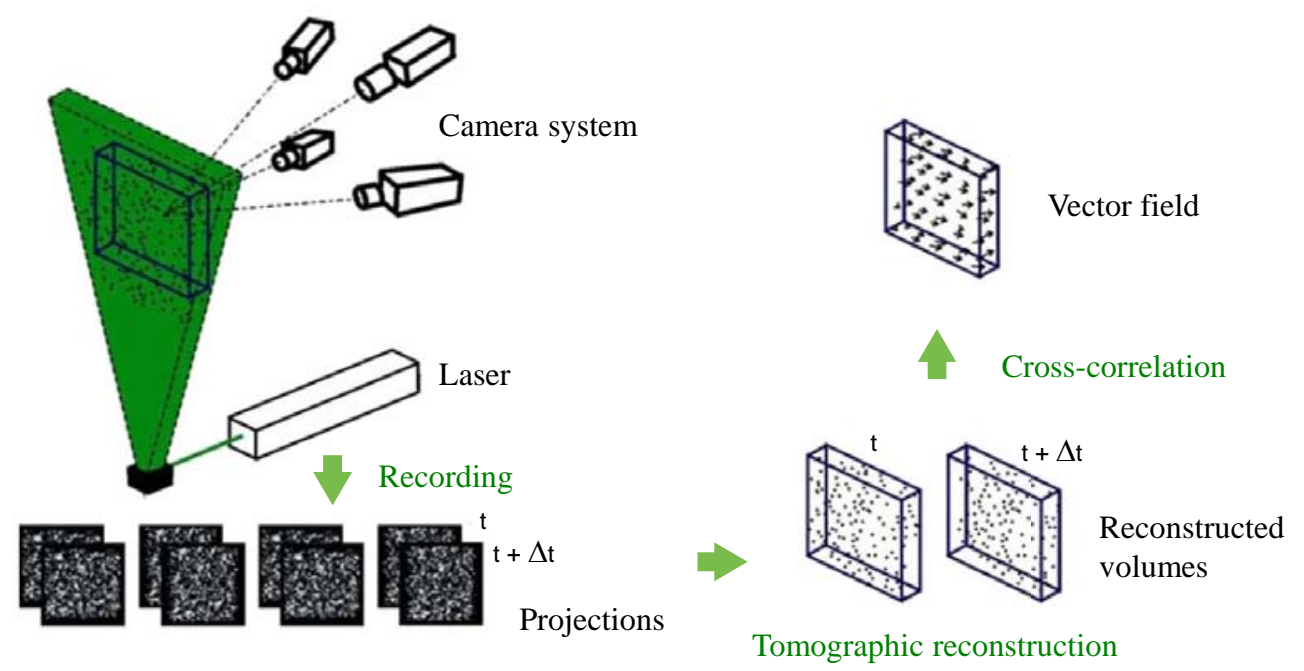

Figure 3.3: Working principle of tomographic PIV (adapted from [Elsinga et al., 2006]).

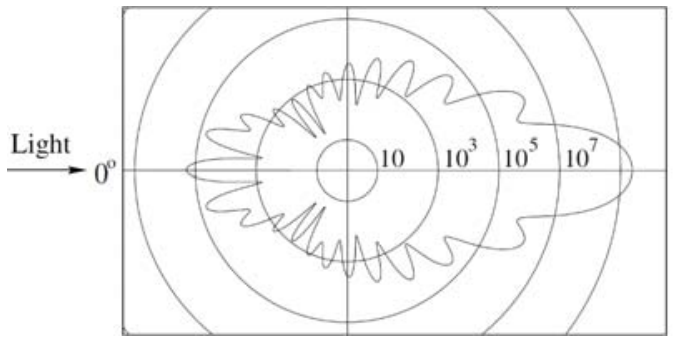

(a)

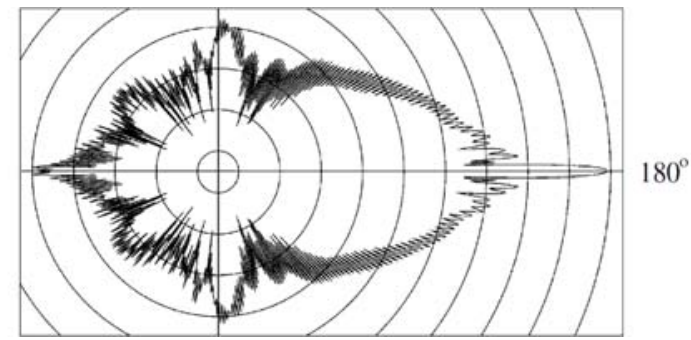

(b)

Figure 3.4: Light scattered by (a) $1 \mu \mathrm{m}$ oil particle in air and (b) $10 \mu \mathrm{m}$ glass particle in water employing a wavelength $\lambda$ of $532 \mathrm{~nm}$ (adapted from [Raffel et al., 2007]). The polar distribution of the scattered light intensity is in logarithmic scale.

\subsubsection{Particle Imaging and Concentration}

The choice of tracer particles is a critical aspect of every PIV, since the flow velocity is computed indirectly from the particle displacements. Particle tracers should be capable to follow the surrounding flow without affecting its behaviour.

In addition, the light scattered from these particles should be clearly detectable on the camera images. The light scattered by small particles depends on the ratio between the particle refractive index and that of the surrounding medium, the particle size, its shape, orientation, polarization and observation angle ([Raffel et al., 2007]). Figure 3.4, extracted from [Raffel et al., 2007], shows two samples of the light scattering by spherical particles according to the Mie's theory ([Hulst and Van De Hulst, 1957]).

The particles are usually imaged in focus, so the focal depth $\delta_{z}$ should be 
larger or equal to the depth of field of the illuminated volume. The equation of the focal depth is given by [Adrian, 1991].

$$
\delta_{z}=4\left[\frac{M+1}{M}\right]^{2} f \#^{2} \lambda,
$$

where $\lambda$ is the wavelength of light, $f \#$ is the numerical aperture (lens f-number) and $M$ the image magnification.

For any particle within the focal depth, assuming diffraction limited imaging and a Gaussian intensity distribution of the particle, its recorded image diameter $d_{\tau}$ can be estimated as follows ([Adrian, 1997])

$$
d_{\tau}=\sqrt{\triangle_{\text {pixel }}^{2}+\left[M d_{p}\right]^{2}+d_{\text {diff }}^{2}}
$$

where $\triangle_{\text {pixel }}$ is the pixel size, $d_{p}$ the particle diameter and $d_{\text {diff }}$ the particle image diffraction size given by

$$
d_{\text {diff }}=2.44 f \#[M+1] \lambda .
$$

Outside the focal depth, the particle image can blur by an amount exceeding 20\% ([Adrian, 1991]). For out of focus particles, [Novara, 2013] proposes a correction on the particle recorded image diameter by adding a blur diameter $d_{B}$ as

$$
\begin{gathered}
d_{\tau}=\sqrt{\triangle_{\text {pixel }}^{2}+\left[M d_{p}\right]^{2}+d_{\text {diff }}^{2}+d_{B}^{2}} \\
d_{B}=\frac{\varepsilon_{B} M^{2}}{f \#},
\end{gathered}
$$

where $\varepsilon_{B}$ is the distance between the particle and the focal plane.

[Scarano, 2013] observed that the relaxation on the $\delta_{z}$ condition, in order to have limited out-of-focus particles, did not degrade the reconstruction quality. That fact is particularly relevant for air experiments with large volume illuminations that face insufficient light intensity. In addition, recent advances in the calibration optical transfer function (OTF) ([Schanz et al., 2013a]) admit an even larger out-of-focus effect.

The need of highly seeded flows, to increase the spatial resolution, contrasts with the quality of tomographic reconstruction, that decreases with the seeding density. The seeding density can be given by the amount of particles recorded onto the image (i.e., particles per pixel, $p p p$ ). This measure is quantified by a simple particle-count procedure. However, besides the number of particles, the accuracy of the tomographic reconstruction is also governed by the particle image diameter ([Novara et al., 2010, Scarano, 2013]). So, the source density $N_{s}$ is a better estimate of the seeding density.

The source density is defined as the mean number of particles in a 
cylindrical volume formed by the intersection of the illuminating light sheet with a circle whose diameter $d_{\tau} / M$ is that of the particle image projected back into the fluid ([Adrian and Yao, 1985]). The source density is given by

$$
N_{s}=C \triangle z \frac{\pi}{4}\left[\frac{d_{\tau}}{M}\right]^{2},
$$

where $C$ is the number of particles per volume and $\triangle z$ is the illuminated volume thickness.

The amount of particles per pixel can be converted to the source density by

$$
N_{s}=\operatorname{ppp} A_{p}
$$

where $A_{p}=(\pi / 4)\left(d_{\tau}^{*}\right)^{2}$ is an effective particle image area (in pixel units) and $d_{\tau}^{*}=d_{\tau} / \triangle_{\text {pixel }}$ is the normalized image diameter, which is typically between 1 and 3 ([Scarano, 2013, Westerweel et al., 2013]).

A good balance between accuracy and spatial resolution is obtained with seeding densities up to $0.05 \mathrm{ppp}\left(N_{s}=0.16\right)$, in which the overlap between particles remains negligible for a four-camera system ([Elsinga et al., 2006, Novara et al., 2010]). Accurate tomo reconstruction of denser particle field is still possible slightly beyond this level (e.g., at $N_{s}=0.3$ ) for good quality images, accurate calibration, larger number of cameras and advanced reconstruction methods ([Scarano, 2013, Novara, 2013, Lynch and Scarano, 2014, Schröder et al., 2015]).

\subsubsection{Volume Illumination}

Differently from planar PIV, tomo-PIV requires a larger amount of light due to the illumination of a volume. The measurement volume is generated by expanding the thickness of the light sheet to a typical depth of one quarter the width or length of the field of view (FOV) ([Scarano, 2013]) and the region is twice illuminated by a light pulse.

Once the illuminated thickness can be comparable to the size of the beam exit diameter, simple optical arrangement of just a single cylindrical diverging lens can be employed. Therefore, the use of more optical components and knife-edge filters, to cut the light intensity beyond the nominal thickness of the measurement volume, is seen in practical applications (e.g., [Elsinga, 2008, Atkinson et al., 2011, Novara, 2013], among others).

As the light intensity is inversely proportional to the required thickness of the measurement volume, the light source power is a limiting factor for tomographic experiments in large measurement volumes. In order to overcome this energy limitation, some special arrangements were proposed as a double- 


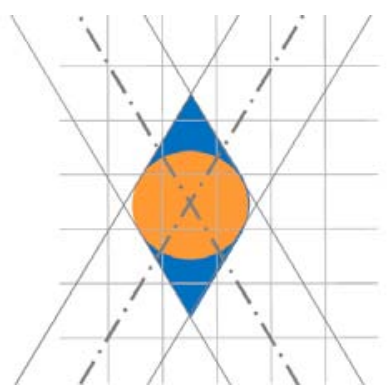

(a)

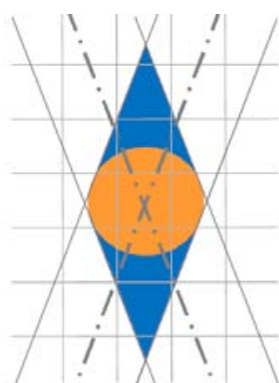

(b)

Figure 3.5: Reconstruction errors due to tomographic angular aperture for a 2-camera system adapted from [Scarano, 2013]. The sketch (a) refers to an angular aperture of $60^{\circ}$ and (b) to an aperture of $40^{\circ}$. The line-of-sights are displayed as dash-dot lines, the actual particles as orange circles and reconstructed particles as blue regions.

pass system ([Scarano and Poelma, 2009]), which uses a plane mirror normal to the illumination axis that reflects the laser light towards the measurement region a second time, and a multi-pass light amplification system ([Schröder et al., 2008a]), in which the circular light beam is sent with a small angle into a region bounded by two mirrors. The largest volumes were obtained in water flows with low-frequency laser systems ([Scarano, 2013]).

\subsubsection{Camera Arrangement}

Tomo-PIV images are commonly acquired by a small number of cameras placed along non-collinear viewing directions. These viewing directions are the key parameters to be considered as they cannot be modified a posteriori, contrarily, they should be optimized during the experimental setup.

The angle between these camera viewing directions is defined as the angular aperture. This angle dictates the spatial resolution along the volume directions. For larger viewing angles, the reconstruction quality degrades due to the longer intersection of the LOS and the measurement volume that increases the chances of particle superposition. Contrarily, smaller viewing angles lead to the reconstruction of elongated particles along the direction of the volume depth ([Scarano, 2013]). This is depicted in figure 3.5, where the actual particle is distorted into a double-cone shape.

The effect of tomographic angular aperture has been studied since [Elsinga et al., 2006]. Lately, [Thomas et al., 2014] investigated the influence of viewing directions in simulated tomo-PIV experiments. In that work, the cameras placed in a circle configuration (cross-like arrangement) produced the best reconstruction results. Nevertheless, the choice of the camera arrangement is frequently dictated by experimental constraints as optical accessibility or 


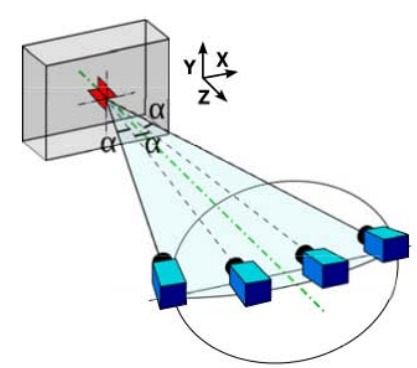

4 cams, single plane

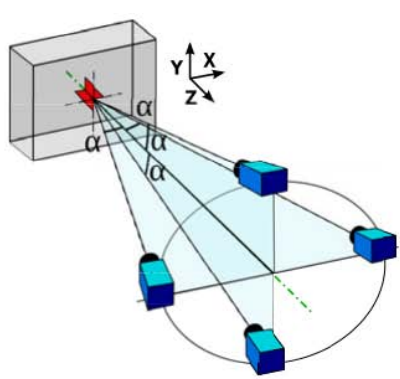

4 cams, circle

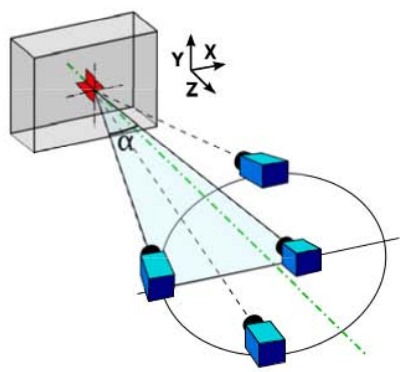

4 cams, 1 cam moving

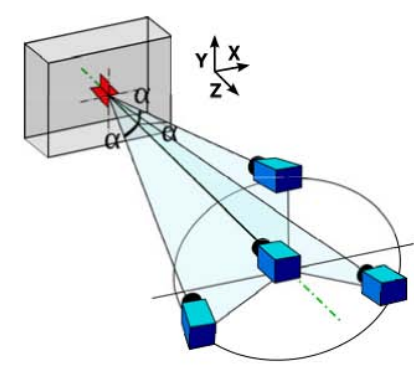

4 cams, central camera

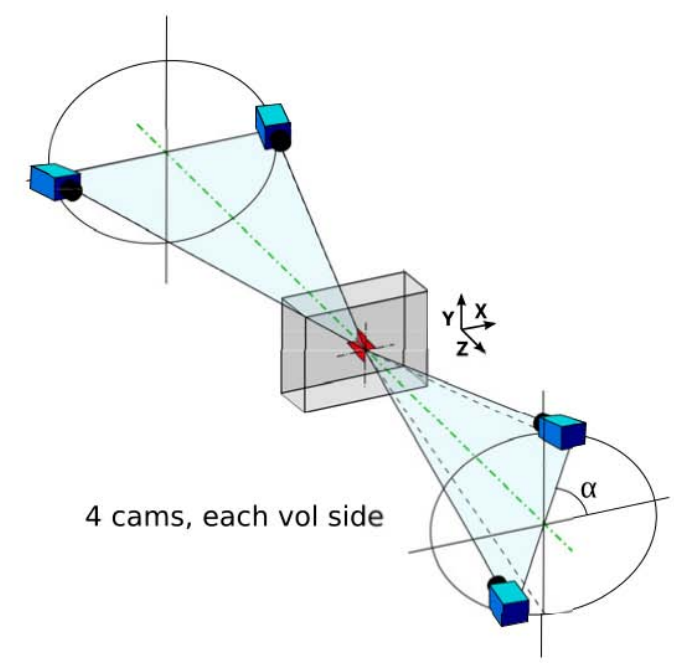

Figure 3.6: Sketch of some camera configurations studied by [Thomas et al., 2014].

intricacy of camera installation.

\subsubsection{System Calibration}

The tomo-PIV reconstruction is based on the relation between the $3 \mathrm{D}$ actual physical space (or world reference system) and the image space. The calibration provides mapping functions ([Prasad, 2000, Willert, 1997]) for the cameras that projects the points in the world reference system $(x, y, z)$ onto the image $i$ coordinate system $\left(u_{i}, v_{i}\right)$. The mapping function can be written as

$$
\left(u_{i}, v_{i}\right)=\Phi_{i}(x, y, z)
$$

The calibration function is obtained by imaging a $2 \mathrm{D}$ target pattern at several positions across the depth direction of the measurement volume or using a multi-layered calibration plate. Although some mapping functions are available in the literature, the third-order polynomial ([Soloff et al., 1997]) and the pinhole model ([Tsai, 1986]) are the most commonly employed. Recently, the calibration optical transfer function ([Schanz et al., 2013a]) became an additional choice. 
The pinhole model is based on the intrinsic parameters (magnifications along $u_{i}$ and $v_{i}$, the skew angle of the sensor matrix and the origin of the camera coordinate system) and extrinsic (rotations and translations) parameters of each camera $i$.

$$
\begin{aligned}
& u_{i}=\frac{a_{11} x+a_{12} y+a_{13} z+a_{14}}{a_{31} x+a_{32} y+a_{33} z+a_{34}} \\
& v_{i}=\frac{a_{21} x+a_{22} y+a_{23} z+a_{24}}{a_{31} x+a_{32} y+a_{33} z+a_{34}}
\end{aligned}
$$

The Soloff's model is more suitable to map complicated refraction geometries (in cases without strongly severe distortions). The model is given by a least-squares polynomial with cubic dependence in $x$ and $y$, and quadratic dependence in the depth $z$.

$$
\begin{aligned}
u_{i}= & a_{0}^{u}+a_{1}^{u} x+a_{2}^{u} y+a_{3}^{u} z+a_{4}^{u} x^{2}+a_{5}^{u} x y+a_{6}^{u} y^{2}+a_{7}^{u} x z \\
& +a_{8}^{u} y z+a_{9}^{u} z^{2}+a_{10}^{u} x^{3}+a_{11}^{u} x^{2} y+a_{12}^{u} x y^{2}+a_{13}^{u} y^{3} \\
& +a_{14}^{u} x^{2} z+a_{15}^{u} x y z+a_{16}^{u} y^{2} z+a_{17}^{u} x z^{2}+a_{18}^{u} y z^{2} \\
v_{i}= & a_{0}^{v}+a_{1}^{v} x+a_{2}^{v} y+a_{3}^{v} z+a_{4}^{v} x^{2}+a_{5}^{v} x y+a_{6}^{v} y^{2}+a_{7}^{v} x z \\
& +a_{8}^{v} y z+a_{9}^{v} z^{2}+a_{10}^{v} x^{3}+a_{11}^{v} x^{2} y+a_{12}^{v} x y^{2}+a_{13}^{v} y^{3} \\
& +a_{14}^{v} x^{2} z+a_{15}^{v} x y z+a_{16}^{v} y^{2} z+a_{17}^{v} x z^{2}+a_{18}^{v} y z^{2}
\end{aligned}
$$

The 3D particle distribution reconstructed by the tomographic technique requires that the lines of sight related to particles imaged by all the cameras are intersecting precisely, considerably stricter than those for stereo-PIV ([Raffel et al., 2007]). A quality tomographic reconstruction requires that each voxel position in space is mapped into a camera pixel position with an error lower than 0.4 pixel ([Elsinga et al., 2006]), preferably lower than 0.1 pixel ([Wieneke, 2008]).

In experiments, mechanical stability of camera holders, tightness of lenstilt adapters, thermal variations within the cameras, vibrations due to the cooling camera systems and vibrations of the experimental test section are usually related to camera pixel errors exceeding 1 pixel ([Scarano, 2013]). Due to the above mentioned conditions, the alignment between the lines of sight from each camera should be corrected. A volume self-calibration procedure for misalignment detection and correction for 3D measurements has been proposed by [Wieneke, 2008].

The volume self-calibration technique is based on the correction of the mapping functions using the particle images ([Wieneke, 2008]). The technique consists in locating the most brightness particles in the camera images $(u, v)$, paring the particle images from each camera and finding the $3 \mathrm{D}$ world position of matching particles $(x, y, z)$ through triangulation, as in Particle Tracking Velocimetry ([Raffel et al., 2007]). The residual triangulation error, namely disparity, obtained by computing the distance between the projection of each 
particle $\left(u_{i}^{\prime}, v_{i}^{\prime}\right)$ and the correspondent positions on the camera images $\left(u_{i}, v_{i}\right)$ as

$$
\boldsymbol{d}_{i}=\left(u_{i}^{\prime}, v_{i}^{\prime}\right)-\left(u_{i}, v_{i}\right)
$$

All particle disparities $\boldsymbol{d}_{i}$ throughout the volume are taken into account to correct the mapping functions $\Phi_{i}$ by means of

$$
\Phi_{i}^{\prime}(x, y, z)=\Phi_{i}(x, y, z)-\boldsymbol{d}_{i}(x, y, z) .
$$

The application of this method works well in low density particle images, which can be recorded before the experimental runs or can be artificially produced by taking only the brightest particle images. The self-calibration procedure can be enumerate as follows ([Wieneke, 2008]).

1. Measurement of $2 \mathrm{D}$ peaks $\left(u_{i}, v_{i}\right)$ of brightest particles in all camera images.

2. Determination of the probable 3D particle positions $\left(x_{i}, y_{i}, z_{i}\right)$ by triangulation.

3. Division of the illuminated volume into sub-volumes.

4. Plotting of the disparities of all paired particles in a sub-volume as a $2 \mathrm{D}$ disparity map.

5. Summation of disparity maps for each sub-volume and each camera over many recordings to achieve better statistics.

6. Detection of the highest disparity peak, for each disparity map, which represents the most probable disparity vector $\boldsymbol{d}_{i}(x, y, z)$.

7. Validation of the 3D-2C disparity vector field $\boldsymbol{d}_{i}$ with replacement of spurious vectors.

8. Correction of the mapping functions $\Phi_{i}$ according to eq. (3.2.12) for all cameras;

9. Repetition of the entire procedure for further improvement.

Figure 3.7(a), from [Wieneke, 2008], sketches the first step of the selfcalibration process that consists of locating 2D particle positions in the camera images and detecting the correspondent 3D particle positions by triangulation for a three-camera system tomo-PIV. Figure 3.7(b) shows the plot of disparities of all particles within a sub-volume of one camera as a 2D disparity map for an actual tomographic PIV experiment of the present work (chapter 7). The disparity peak appears to be at the bottom-left of the graph in this example. 


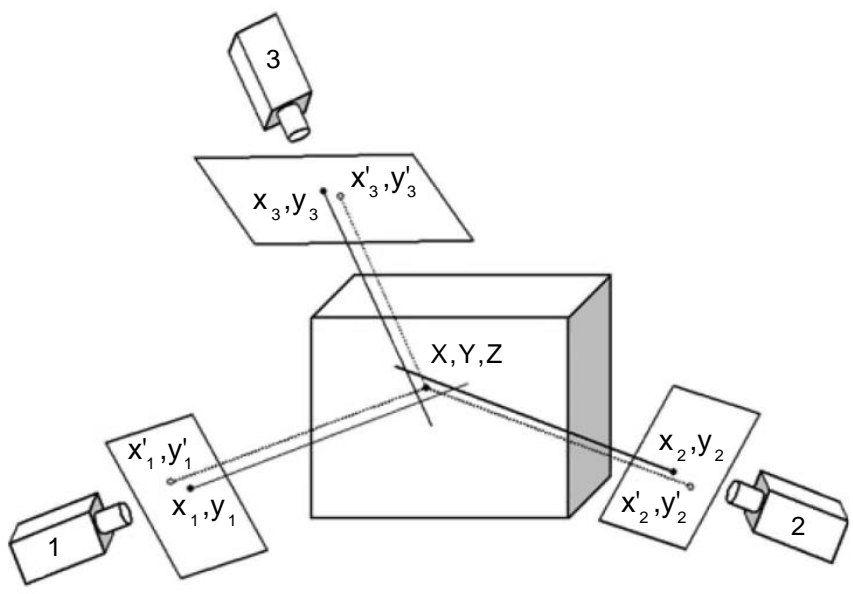

(a)

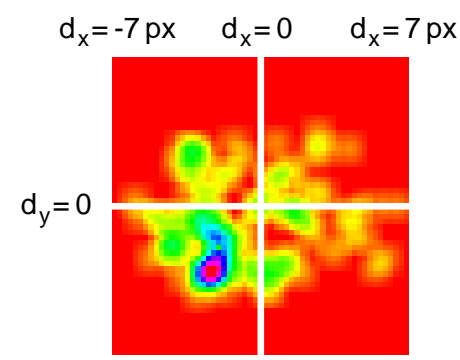

(b)

Figure 3.7: (a) Residual triangulation disparities $\boldsymbol{d}_{i}(X, Y, Z)=\left(x_{i}, y_{i}\right)-\left(x_{i}^{\prime}, y_{i}^{\prime}\right)$ due to calibration errors. (b) Sample of a disparity map of one of the cameras for a single sub-volume. (Adapted from [Wieneke, 2008].)

\subsubsection{Reconstruction Techniques}

The tomo-PIV is based on the reconstruction of the 3D particle distribution by optical tomography introduced by [Elsinga et al., 2006]. The tomographic volume is a 3D array of voxels that discretizes the physical space. The grey intensity of each voxel $\boldsymbol{E}(x, y, z)$ is calculated from the pixel intensity of the camera images $\boldsymbol{I}_{k}\left(u_{i}, v_{i}\right)$ by algebraic methods ([Herman and Lent, 1976]). The relation considering the superposition can be written as follow:

$$
\sum_{j \in L_{i, k}} w_{i, j, k} \boldsymbol{E}\left(x_{j}, y_{j}, z_{j}\right)=\boldsymbol{I}_{k}\left(u_{i}, v_{i}\right)
$$

where $L_{i, k}$ represents the voxels intercepted by the line of sight (LOS) of the $i^{\text {th }}$ pixel in the $k^{\text {th }}$ camera together with their close neighbours (typically within one kernel $3 \times 3$ centred on the intersected voxel) and $w_{i, j, k}$ is a weight function related to the contribution of the $j^{\text {th }}$ voxel $\left(x_{j}, y_{j}, z_{j}\right)$ in these pixels.

The weighting function is calculated as the volume fraction from the intersection between the voxel and the line of sight. Considering the estimation of the intersection between a pyramid emerging from the camera pixel and a cubic voxel is mathematically challenging, other weighting functions were proposed ([Thomas et al., 2010, Schanz et al., 2013a, Thomas et al., 2014]). The usually estimation of the volume intersection is obtained assuming each pixel line of sight as a cylinder and each voxel as a sphere ([Lamarche and Leroy, 1990]). Figure 3.8 illustrates the particle distribution reconstructed volume as an array of voxels discretizing the physical space ([Scarano, 2013]). In the figure, the voxels falling within the shaded stripe (weighted cross section) have 


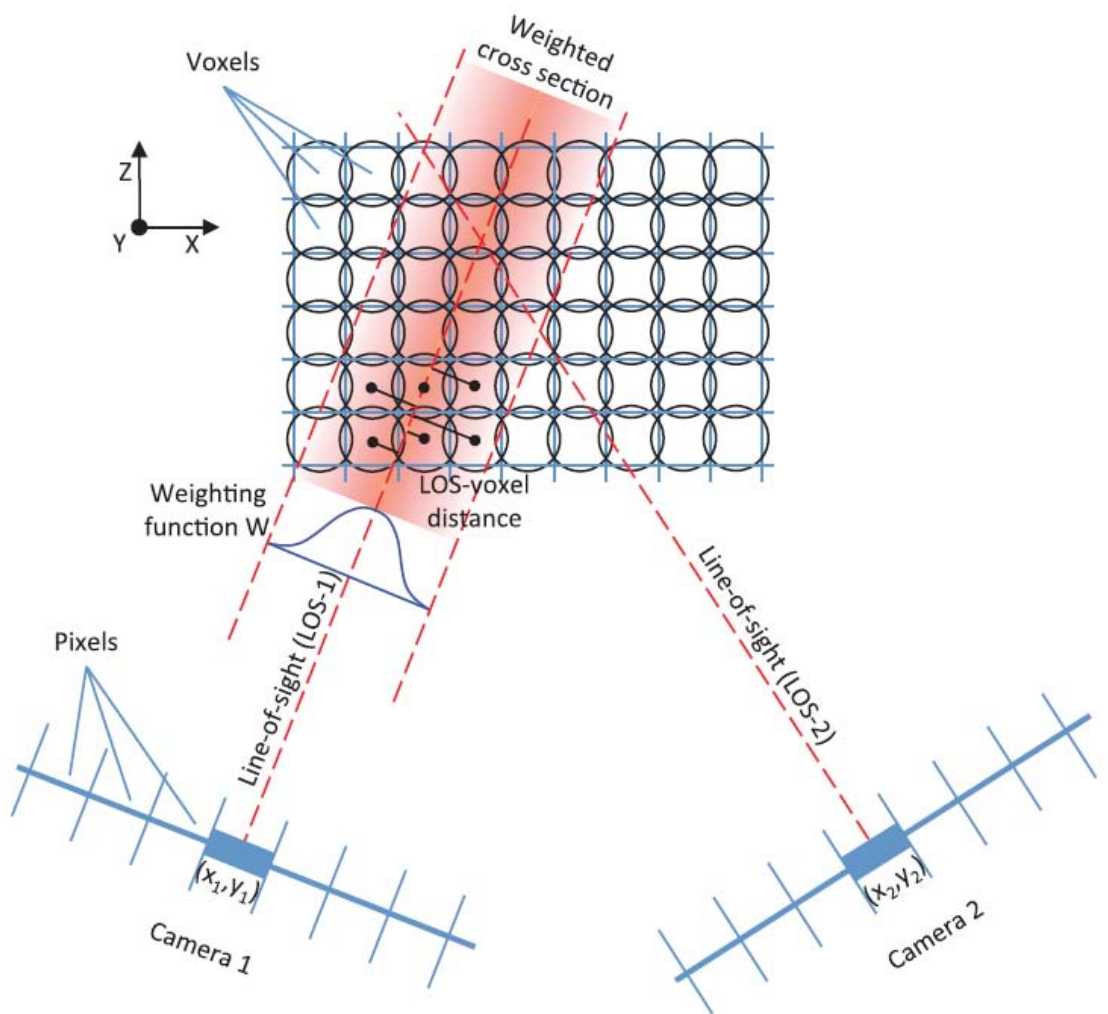

Figure 3.8: Volume reconstruction from two-camera tomographic PIV reproduced from [Scarano, 2013].

non-zero value of the weighting coefficient $w_{i, j, k}$, the others the coefficient is computed from the intersection of a sphere by a cylinder .

The solution of the problem proposed by equation 3.2.13 can be achieved by tomographic reconstruction algorithms. The three classical reconstruction algorithms studied in this work, named MART, SMART and BIMART, are described in details as follow.

\section{MART}

For the multiplicative algebraic reconstruction technique (MART) ([Elsinga et al., 2006]), each pixel $i$ in each camera $k$, the intensity of all the voxels $j$ along the line of sight (LOS) are updated iteratively according to

$$
\boldsymbol{E}\left(x_{j}, y_{j}, z_{j}\right)^{n+1}=\boldsymbol{E}\left(x_{j}, y_{j}, z_{j}\right)^{n}\left[\frac{\boldsymbol{I}_{k}\left(u_{i}, v_{i}\right)}{\sum_{j \in L_{i, k}} w_{i, j, k} \boldsymbol{E}\left(x_{j}, y_{j}, z_{j}\right)^{n}}\right]^{\mu w_{i, j, k}}
$$

where $n$ indicates the iteration, $w_{i, j, k}$ the voxel weight and $\mu$ is a relaxation parameter.

Firstly, the volume is initialized by a multiplicative line-of-sight approach (MLOS) ([Atkinson and Soria, 2009]) or by a minimum line-of-sight approach 
(MinLOS) ([Putze and Maas, 2008]) in order to accelerate the time-consuming process of iterative MART reconstruction ([Thomas et al., 2014]). The principle consists by the fact that the particle distribution in the reconstructed volume is sparse and only voxels corresponding to non-zero intensity are updated from the following iterations. The number of iterations required to achieve a high quality reconstruction volume can vary significantly from each reconstruction algorithm. The use of 5 iterations seems to be a consensus for the standard MART ([Elsinga et al., 2006]), although a thorough study of convergence is recommended for each experimental setup.

\section{SMART}

For the simultaneous multiplicative algebraic reconstruction technique (SMART), which was first used by [Atkinson and Soria, 2009], voxel intensities are updated based on the simultaneous projection to every pixel, contrariwise to MART that corrects voxels based on only one pixel at a time. The procedure is governed by the following expression

$\boldsymbol{E}\left(x_{j}, y_{j}, z_{j}\right)^{n+1}=\boldsymbol{E}\left(x_{j}, y_{j}, z_{j}\right)^{n}\left[\prod_{i, k \in N_{j}}\left[\frac{\boldsymbol{I}_{k}\left(u_{i}, v_{i}\right)}{\sum_{l \in L_{i, k}} w_{i, l, k} \boldsymbol{E}\left(x_{l}, y_{l}, z_{l}\right)^{k}}\right]^{\mu w_{i, j, k}}\right]^{1 / N_{j}}$,

where $N_{j}$ represents the pixels that have a LOS crossing a given voxel $j$.

\section{BIMART}

This block-iterative MART (BIMART) proposed by [Byrne, 2009] is a generalization of both MART and SMART where each image is divided into $Q$ blocks ([Thomas et al., 2014]. In the limit, if the block size is larger than the image size, the algorithm becomes SMART. Conversely, if the block size is equal to one pixel, the algorithm becomes MART. The intensity of each voxel is updated according to:

$$
\boldsymbol{E}\left(x_{j}, y_{j}, z_{j}\right)^{n+1}=\boldsymbol{E}\left(x_{j}, y_{j}, z_{j}\right)^{n} \prod_{i, k \in B_{Q} \cap N_{j}}\left[\frac{\boldsymbol{I}_{k}\left(u_{i}, v_{i}\right)}{\sum_{l \in L_{i, k}} w_{i, l, k} \boldsymbol{E}\left(x_{l}, y_{l}, z_{l}\right)^{n}}\right]^{\mu w_{i, j, k}},
$$

where $B_{Q}$ contains the pixels inside the block. 


\subsubsection{D Motion Analysis}

The standard method employed to extract the displacement field from a pair of reconstructed volumes is based on 3D cross-correlation. The measurement domain is divided into several interrogation volumes and the following operations are applied, as summarized by [Scarano, 2013].

1. Computation of the 3D fast Fourier transform (FFT) of both corresponding interrogation volumes.

2. Evaluation of the cross-product of the first interrogation volume FFT and the conjugate of the second interrogation volume FFT.

3. Computation of the inverse FFT of the result.

4. Location of the maximum of the cross-correlation.

5. Estimation of sub-pixel displacement based on the neighbours around the maximum value.

6. Computation of velocity employing the magnification factor and the time separation between exposures.

The application of the FFT algorithm is not mandatory to compute the cross-correlation, but it represents a faster alternative to the direct application of the algebraic cross-correlation operator. The normalized cross-correlation of the reconstructed intensity fields is given by eq. (3.2.17). A local interpolation around the peak value of the cross-correlation field provides a peak position with sub-pixel precision. The Gaussian peak fit is still the most adopted scheme ([Raffel et al., 2007]).

$\boldsymbol{R}(\triangle x, \triangle y, \triangle z)=\frac{\sum_{x} \sum_{y} \sum_{z} \boldsymbol{E}_{1}(x, y, z, t) \cdot \boldsymbol{E}_{2}(x+\triangle x, y+\triangle y, z+\triangle z, t+\triangle t)}{\sqrt{\operatorname{std}\left(\boldsymbol{E}_{1}(t)\right) \cdot \operatorname{std}\left(\boldsymbol{E}_{2}(t+\triangle t)\right)}}$,

where $\boldsymbol{E}_{1}$ and $\boldsymbol{E}_{2}$ represent windows of the reconstructed volume containing particle distribution intensities (after mean intensity subtraction) at positions $(x, y, z)$ and time $t, \Delta t$ is the time interval that separates the two exposures, $(\triangle x, \triangle y, \triangle z)$ is the $3 \mathrm{D}$ space shift and std refers to the standard deviation operator.

The measurement displacement accuracy is related manly to the number of actual particle image pairs in the interrogation volumes, the velocity gradient within the volume and the particle image size with respect to their spatial 
discretization ([Scarano, 2013]). To increase the signal-to-noise ratio in the 3D cross-correlation and avoid peak looking effects ([Raffel et al., 2007]), it would be desirable to have a particle diameter of $2<d_{i}<3$ voxels ([Michaelis et al., 2015]) and a number of paired particles within an interrogation volume between 5 and 10 ([Novara, 2013]).

The computational cost for cross-correlation analysis in tomographic PIV is about four to five orders of magnitude higher than in planar PIV ([Scarano, 2013]). Recently, several algorithms using a binning approach ([Discetti and Astarita, 2012]), the sparse characteristic of reconstructed volumes ([Atkinson and Soria, 2009]), direct correlation of small search radius at advanced stages of the multi-pass interrogation ([Scarano, 2013]), multiple 2D FFT calculations instead of 3D FFT ([Earl et al., 2015]) or particle tracking based algorithms able to cope with high seeding densities as STB ([Schanz et al., 2014]), to mention just a few, were developed in order to accelerate this time consuming displacement calculation and to improve the accuracy.

\subsection{Quality Indicators}

In actual experiments, normally it is not possible to compare reconstructed particle locations or a velocity field with a reference result in order to estimate the measurement accuracy, as usually practiced in studies employing synthetic-image simulations. Furthermore, optimized parameters, such as for image pre-processing, reconstruction or correlation algorithms, based only on simulations can deviate from parameters obtained for actual experiments. Error estimations from these simulations are typically much lower than those encountered in experiments ([Atkinson et al., 2011]). Currently, users rely on previous know-how to set the parameters for a particular experiment, evaluating the actual quality of the results obtained after the experiments are concluded ([Westerweel et al., 2013]). It would be desirable that the quality of the reconstruction and its accuracy could be estimated before the start of a time-consuming processing campaign ([Scarano, 2013]) or, even better, during the measurements phase.

The use of quality indicators for optimization purposes can be useful at two stages of the experimental campaign. Firstly, in the preliminary phase of tests with the experimental setup, before acquiring the complete data set, when adjustments of the tomo-PIV physical parameters are still possible. The quality indicators can also be useful before the reconstruction phase of each experimental data set, in order to set the best tomo-PIV software parameters to process the data. 
The next sections present a description of some frequently used reconstruction quality criteria. When no ground truth data are available, the light intensity signal-to-noise ratio, the normalized intensity variance and the reconstructed particle characteristics can be used as quality indicators of the tomo reconstruction. On the other hand, when synthetic data is available, all quality indicators described below can be used as a tool to check the algorithm performance. A high quality reconstruction can be achieved employing multiple cameras, as the 12-camera experimental study of [Lynch and Scarano, 2014], and, in such cases, the reconstructed volume and velocity field can be adopted as a reference for optimization purposes.

It should be mentioned that reconstruction quality indicators do not offer absolute values that can be used for accessing the performance of reconstruction algorithms. For that reason, a proper analysis of the quality of a reconstruction should balance the combined information yielded by different indicators as, for instance, those that will be presented next.

\subsubsection{Standard Reconstruction Quality}

To quantify the accuracy of the reconstruction process, the reconstructed intensity $\boldsymbol{E}_{1, j}$ in the volume position $\left(x_{j}, y_{j}, z_{j}\right)$ is compared with the exact distribution of light intensity $\boldsymbol{E}_{0, j}$ where the particles are represented by a Gaussian intensity distribution. From now on, the extended form $\boldsymbol{E}\left(x_{j}, y_{j}, z_{j}\right)$ is simplified to $\boldsymbol{E}_{j}$ along the text. The standard reconstruction quality $Q_{\text {rec }}$ is defined as the normalized correlation coefficient of the exact and reconstructed intensity distributions ([Elsinga et al., 2006]).

$$
Q_{r e c}=\frac{\sum_{j} \boldsymbol{E}_{1, j} \cdot \boldsymbol{E}_{0, j}}{\sqrt{\sum_{j} \boldsymbol{E}_{1, j}^{2} \cdot \sum_{j} \boldsymbol{E}_{0, j}^{2}}}
$$

The tomo reconstruction should be considered sufficiently accurate for a correlation coefficient $Q_{\text {rec }}>0.75$ ([Elsinga et al., 2006]). Unfortunately, the reconstruction quality can only be used when the exact particle distribution is known, i.e., in synthetic simulations.

It should be mentioned that the standard reconstruction just presented can yield values below 1 even for a perfect reconstruction. This might happen in the case of reconstructed particle sizes being different from the synthetic baseline values. In this case, the size of reconstructed particle indicator that will be presented in section 3.3.6 should be considered together with the standard indicator. 


\subsubsection{Re-Projection Quality}

The re-projection quality is computed as the normalized correlation between the intensities of the original particle images $I_{k, i}=I_{k}\left(u_{i}, v_{i}\right)$ and the re-projections of the reconstructed volume onto the camera sensor planes $I_{k, i}^{\prime}([$ Thomas et al., 2014]).

$$
Q_{\text {reproj }}^{k}=\frac{\sum_{i} \boldsymbol{I}_{k, i} \cdot \boldsymbol{I}_{k, i}^{\prime}}{\sqrt{\sum_{i} \boldsymbol{I}_{k, i}^{2} \cdot \sum_{i} \boldsymbol{I}_{k, i}^{\prime 2}}}
$$

Different from the normalized correlation coefficient proposed by [Elsinga et al., 2006], the averaged re-projection quality in all cameras, $<Q_{\text {reproj }}>$, does not characterize the accuracy of the volume reconstruction, due to the fact that ghost and actual particles can superimpose themselves in the re-projection images. Even though this criterion is unable to infer the reconstruction quality, it is a useful tool to check the calibration and the convergence of the algorithm ([Thomas et al., 2014]).

\subsubsection{Particle Distribution Signal-to-Noise Ratio}

An indicator of the signal-to-noise ratio $\left(S N R_{N}\right)$ in the tomographic reconstruction of the $3 \mathrm{D}$ particle distribution can be given as the ratio between the number of actual tracer particles $N_{p}$ and the number of ghost particles $N_{g}$ ([Elsinga et al., 2006, Novara et al., 2010]). The $S N R_{N}$ can be associated with the number of cameras $N_{c}$, an effective particle image area in pixel squared $A_{p}$, the typical length of the LOS crossing the measurement volume $\ell_{z}$ (approximately the volume depth) and the source density $N_{s}$ (proposed by [Adrian, 1991]).

$$
S N R_{N}=\frac{N_{p}}{N_{g}}=N_{s}^{N_{c}-1} A_{p}^{-1} \ell_{z}^{-1}
$$

For $S N R_{N}>1$, velocity bias errors from ghost particles are relatively small and appear to decrease even with increasing particle image density ([Elsinga et al., 2011, Westerweel et al., 2013]). Nevertheless, the particle density should be under the speckle limit $N_{s}<0.4$ ([Westerweel et al., 2013]). Beyond that limit, too many pixels have non-zero intensity and the tomographic reconstruction is highly undetermined.

The particle distribution signal-to-noise ratio is a conservative estimation of reconstruction and cross-correlation performances ([Lynch and Scarano, 2014]) since it takes into account only the number of ghost particles formed, and not their intensity. Numerous ghost particles do not imply a failure of the velocity measurement, due to the fact that the intensity of a ghost particle 
seldom approaches actual particle intensities, which causes them to contribute less to the cross-correlation ([Elsinga et al., 2006, Elsinga et al., 2011, Scarano, 2013, Westerweel et al., 2013]). A complementary metric is the light intensity signal-to-noise ratio ([Scarano, 2013, Lynch and Scarano, 2014]).

\subsubsection{Light Intensity Signal-to-Noise Ratio}

As the intensity profile of the reconstructed light must agree with the experimental light profile, it should be used to estimate the quality of the reconstruction (e.g., [Elsinga et al., 2006, Scarano and Poelma, 2009, Atkinson and Soria, 2009, Fukuchi, 2012, Scarano, 2013, Lynch and Scarano, 2014]). The method requires a "top-hat" illumination of an uniform seeded measurement domain. The intensity levels outside the illuminated region are not zero because of ghost particles, multiple scattering and reflections, which are referred to as "noise", whereas inside the reconstructed region actual particles and noise are formed. This difference associated with the reconstructed intensity allows the estimation of the light intensity signal-to-noise ratio, defined by [Scarano, 2013] as the ratio of the averaged reconstructed intensity inside the illuminated region $<\boldsymbol{E}_{\text {in }}>$ to that outside of the region $<\boldsymbol{E}_{\text {out }}>$.

$$
S N R_{E}=\frac{<\boldsymbol{E}_{\text {in }}>}{<\boldsymbol{E}_{\text {out }}>}
$$

[Scarano, 2013] proposed $S N R_{E} \geq 2$ as the lower limit identifying tomographic experiments with reconstruction of good quality, eventually leading to a reliable measurement analysis. Figure 3.9 shows a sample of the $S N R_{E}$ calculation in a profile of reconstructed light by MART from the boundary-layer experiment. $<\boldsymbol{E}_{\text {in }}>$ was computed by the average intensity inside the investigation region (blue circles) while $<\boldsymbol{E}_{\text {out }}>$ was calculated by the average of points inside the "valley" (red triangles). Higher intensity values close to the volume borders should not be considered since they overestimate the noise. It is best practice in the tomo-PIV reconstruction a volume enlargement along the thickness, to increase the quality, that is discarded before the cross-correlation procedure ([Thomas et al., 2014]).

were result of an artefact of the reconstruction and should be discarded (is recommended in order to will (as mentioned in )

Additionally, the variations of $\left\langle\boldsymbol{E}_{\text {in }}>\right.$ and $\left\langle\boldsymbol{E}_{\text {out }}>\right.$ along the iterative reconstruction process can be used as a tool to check the convergence of the tomo-PIV algorithm. 


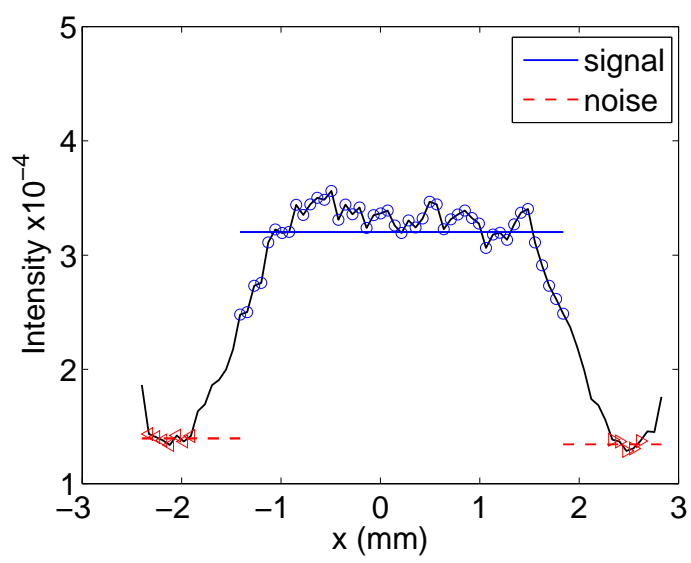

Figure 3.9: Sample of normalized intensity profile of reconstructed light by MART from the boundary-layer experiment.

\subsubsection{Normalized Intensity Variance}

Another metric of reconstruction quality considers the normalized variance of the reconstructed intensity within the investigated volume, as proposed by [Novara and Scarano, 2012]. This metric, presented in eq. (3.3.5), does not require an uniform illumination, neither the reconstruction of the non-illuminated region. A high value of the normalized variance indicates a sparse reconstructed field with high-amplitude peaks. In contrast, a low value is associated with a denser distribution of intensity with lower-amplitude peaks associated with ghost particles. [Lynch and Scarano, 2014] suggested a normalized intensity variance value $\sigma_{E}^{*}>20$ leading to an acceptable reconstruction quality. They encourage the use of this criterion as a complementary tool to the $S N R_{E}$, although having lower sensitivity to quality variations.

$$
\sigma_{E}^{*}=\frac{\sqrt{\frac{1}{N} \sum_{j}^{N}\left(\boldsymbol{E}_{j}-<\boldsymbol{E}_{\mathrm{in}}>\right)^{2}}}{<\boldsymbol{E}_{\mathrm{in}}>},
$$

where $N$ is the amount of voxels inside the 3D measurement region.

\subsubsection{Reconstructed Particle Characteristics}

The reconstructed particles should be expected to have a Gaussian shape, since the particle images are very often diffraction-limited spots as a result of discretization ([Discetti et al., 2013]). Sometimes, elongation of actual particles in the depth direction appears especially when the total viewing angular aperture is too small, increasing random errors in the measurement of the velocity component along the depth ([Novara et al., 2010]).

As mentioned previously in section 3.2, the accuracy of the tomo-PIV reconstruction depends on the angular aperture. For higher values of the viewing 
angle, the reconstruction quality decreases due to the longer intersection of the LOS across the measurement volume that rises the probability of particle superposition. Otherwise, smaller aperture angles lead to the formation of elongated particles along the volume thickness (please revisited figure 3.5). An appropriate estimate of the particle shape can be made by the optical transfer function as proposed by [Schanz et al., 2013a].

The particle elongation can be compensated only by proper orientation of the cameras (optimizing the aperture angles in the camera setup), but can be attenuated by filtering (e.g., SFIT), using a proper reconstruction algorithm (e.g., classical algorithms with enough reconstruction iterations) or a OTF as a weighting function (to account for non-uniform distortions). An estimation of particles roundness in the entire reconstructed volume can be done by means of an autocorrelation procedure. The particle intensity can be considered as Gaussian and the diameter can be defined as four times the standard deviation, which comprises $95 \%$ of the peak intensity. The diameter in each direction $i$ is computed by the average of standard deviations $\sigma$ of a 3-point Gaussian peak fit in the interrogation volumes.

$$
d_{i}=4<\sigma_{i}>
$$

For a good 3D cross-correlation ([Raffel et al., 2007]) it would be desirable to have an equivalent particle dimension along all directions, $d_{x} \cong d_{y} \cong d_{z}$, and a diameter size of $2<d_{i}<3$ voxels.

It is worth mentioning that the major contribution to the correlation comes from the actual particles, due to their generally higher intensities as compared to ghost particles, as mentioned before. This approach, which seems to be used here for the first time in tomo-PIV for size quantification, is not valid for high seeding densities.

In addition to the particle diameter, the intensity of the reconstructed particles can be assessed and its histogram can be compared to the particle intensities of the tomo-PIV images. Due to the use of pre-, intermediateand post-processing filtering and the reconstruction algorithm employed, the particle intensities inside the reconstructed volume can be affected and, as a consequence, the performance of the 3D cross-correlation.

Another important particle characteristic is the amount of particles per volume $(p p v)$. Again in this case, besides the reconstruction method, the filters employed can change significantly the $p p v$ influencing the velocity field resolution. 


\subsubsection{Error in Particle Position}

For a given synthetic study, the 3D particle position is known and the error between the actual reconstructed particles and the reference can be used as a quality indicator (e.g., [Novara et al., 2010, Schanz et al., 2013a, Schanz et al., 2014]). The mean error norm can be computed from the synthetic particle position, $\boldsymbol{x}_{0}=\left(x_{0}, y_{0}, z_{0}\right)$, and the corresponding reconstructed particle centres, $\boldsymbol{x}_{1}=\left(x_{1}, y_{1}, z_{1}\right)$, of all actual particles, $N_{p}$. The symbol $\|\cdot\|$ is related to the Euclidean norm operator.

$$
\epsilon(\boldsymbol{x})=\frac{1}{N_{p}} \sum_{i=1}^{N_{p}}\left\|\boldsymbol{x}_{1, i}-\boldsymbol{x}_{0, i}\right\|
$$

\subsubsection{Error in Velocity Measurements}

When a synthetic data set is provided, the uncertainty in velocity measurements can be calculated from the ground truth displacement or velocity field (e.g., [Elsinga et al., 2006, Worth and Nickels, 2008, Michaelis et al., 2010, Atkinson et al., 2011, Bilsky et al., 2011, Schanz et al., 2013a, Lynch and Scarano, 2014]). The mean error norm can be calculated from the difference of the synthetic velocity field, $\boldsymbol{u}_{0}=\left(u_{0}, v_{0}, w_{0}\right)$, and the corresponding velocity field computed from the pair of reconstructed volumes, $\boldsymbol{u}_{1}=\left(u_{1}, v_{1}, w_{1}\right)$, of all vectors, $N_{v}$.

$$
\epsilon(\boldsymbol{u})=\frac{1}{N_{v}} \sum_{i=1}^{N_{p}}\left\|\boldsymbol{u}_{1, i}-\boldsymbol{u}_{0, i}\right\|
$$

In addition to the velocity error indicator, the amount of spurious vectors could be used as a complementary information of the velocity measurement quality ([de Silva et al., 2013]).

\subsubsection{Uncertainty in Velocity Measurements}

For an experimental investigation, there is yet no consensus about the estimation of PIV uncertainty ([Sciacchitano et al., 2014]). In the present study, the uncertainty on the velocity field is estimated from the analysis of the local velocity-fluctuation divergence ([Moffat, 1988, Atkinson et al., 2011]). From the $3 \mathrm{C}-3 \mathrm{D}$ velocity field, the velocity gradients in the $x, y$ and $z$ directions can be computed by a second-order central difference scheme ([Foucaut and Stanislas, 2002]) to access both the velocity gradient tensor and the divergence of the fluctuating velocity. Since, for an incompressible flow without error measurements, the divergence of the velocity must be zero, the uncertainty 
in the divergence $\delta\left({ }^{\partial u_{i}^{\prime}} / \partial x_{i}\right)$ can be computed as the root-mean-square of the divergence. Assuming that the vector spacing $\triangle_{\text {vec }}$ and the uncertainty in each direction are uniform, the uncertainty on the velocity $\delta(\boldsymbol{u})$ can be given as ([Atkinson et al., 2011])

$$
\delta(\boldsymbol{u})=\sqrt{\frac{2 \triangle_{\mathrm{vec}}^{2}}{3}} \delta\left(\frac{\partial u_{i}^{\prime}}{\partial x_{i}}\right) .
$$

The divergence of the fluctuating velocity is influenced by the size of the correlation volume. A smooth velocity field computed from a poor quality reconstruction volume populated by ghost particles can present small divergence, while a velocity field calculated by an undersized interrogation volume of well reconstructed particle distribution volumes can produce high divergence. For that reason, the uncertainty in velocity measurements computed by the divergence is useful when combined with some quality indicators, mentioned previously, and an interrogation volume size study, as, for example, that proposed by [Foucaut et al., 2012b].

\subsubsection{Experimental Optimization Using Quality Indic- ators}

The quality indicators described in the preceding sections can be used to help optimize an actual tomo-PIV experiment, as will be outlined now. In the description that follows, it is assumed that the physical tomo-PIV setup has already been designed and carried out, according to the best practices proposed in the literature (e.g., [Raffel et al., 2007, Scarano, 2013]).

As common practice, preliminary tomo-PIV images of the flow configuration under study are acquired using low-particle seeding density. A few samples of these low-particle-density images can be used for both, self-calibration implementation ([Wieneke, 2008]), and initial tests of the reconstruction procedures. Since the low-particle-density images reduce the amount of ghost particles inside the reconstruction volume ([Maas et al., 1993]), they produce satisfactory results even when using non-optimized tomo-PIV reconstruction parameters, chosen based on studies with synthetic data, or simply based on previous experience.

The small set of low particle density images can be used at this early stage of the experimental campaign to optimize the reconstruction parameters. To this end, the light intensity signal-to-noise ratio and the normalized variance quality criteria can be used. Also the quality of the reconstruction can be verified by checking the reconstructed particle characteristics. As already mentioned, it is important that the output from these criteria be analysed 
in conjunction.

In the event that the optimized reconstruction fails to meet the quality criteria by yielding values outside the recommended ranges, a modification of the experiment setup is recommended. This procedure would avoid that complete data sets with higher particle densities be processed at a high computational cost to reveal that the data display unacceptable quality.

If the quality criteria are obtained for the low particle density tests, one can proceed to the next experimental phase where sequences of images with higher seeding densities are acquired. It is important to have in mind that there is a compromise between the quality of the reconstructed volumes and the resolution of the velocity field obtained, indirectly given by the seeding density. Again, in order to save computational effort, only a few image samples of the complete data set are reconstructed and used for the optimization of the tomo-PIV parameters. The same quality indicators mentioned in the previous paragraph regarding the low-particle-density runs should be applied here. After the optimization procedure, the whole data set can be reconstructed employing the optimized parameters obtained. Significant savings of computer time and good quality velocity fields can be obtained by the procedure just outlined. Optimization of the cross-correlation procedures and velocity field post-processing would follow the reconstruction. 


\section{4 \\ Boundary Layer Experiment}

The tomo-PIV in a turbulent boundary-layer experiment was made in the end of 2010 ([Foucaut et al., 2011a, Foucaut et al., 2012a]), before the author went to LML, through the ANR VIVE 3D contract, in the partnership of CORIA (Rouen), Pprime (Poitiers) and LML (Lille). The experiment took place in a large-sized wind tunnel in the LML facilities, which is suitable for high-resolution experiments at high Reynolds numbers.

This chapter describes the wind tunnel with the tomo-PIV system employed. It is also discussed the calibration and self-calibration procedures showing a method of virtually translating planes in order to compensate refraction errors in the images of the calibration target. These calibration procedures were recomputed in the present work, aiming the reduction of projection and triangulations errors, from the target images and low-density particle images obtained from this previously conducted experiment.

\subsection{Wind Tunnel}

The overall length of the wind tunnel, which is sketched in figure 4.1, is about $20 \mathrm{~m}$ to allow the development of the boundary layer. The tunnel cross-section is $1-\mathrm{m}$ high and $2-\mathrm{m}$ wide. The last $5 \mathrm{~m}$ provide optical access from all sides, which allows flexibility for different experiments and optical arrangements. The free stream velocity can be regulated from 3 to $10 \mathrm{~m} / \mathrm{s}$, with stability better than $0.5 \%$, by varying the fan rotation speed. The tunnel can be operated in closed loop with temperature control or open to the outside. In the close-loop configuration, the temperature is kept within $\pm 0.2^{\circ} \mathrm{C}$ by using an air-water heat exchanger, located before the plenum chamber. The boundary layer that develops on the lower wall is tripped at the inlet of the tunnel by a grid laid on the floor. This grid fixes the transition and increases slightly the boundary-layer thickness in the test section, not affecting the turbulent statistics. Corner effects due to the rectangular cross-section of the wind tunnel do not affect the homogeneity of the flow in the transverse direction up to $\pm 350 \mathrm{~mm}$ from the vertical plane of symmetry of the tunnel. Although the 


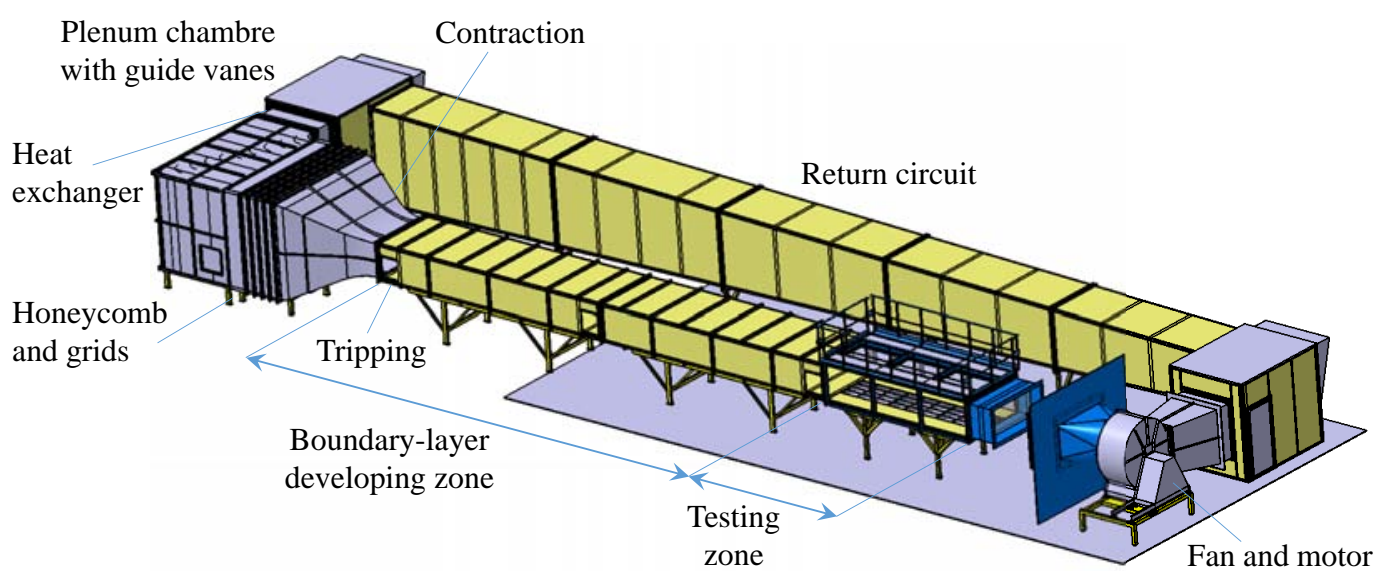

Figure 4.1: LML wind tunnel.

walls of the wind tunnel are parallel, streamwise pressure gradient established is considered negligible. High Reynolds number, based on the momentum thickness, $R_{\theta}$ of 20600 with a boundary layer of about 300-mm thickness and a free stream turbulence intensity of $0.3 \%$ can be achieved in this facility. More information about the wind-tunnel can be found in the [Carlier and Stanislas, 2005].

In the present work, the experiment was carried out at a Reynolds number $R_{\theta}$ equal to 8500 in the closed-loop configuration of the wind tunnel with a temperature of $15^{\circ} \mathrm{C}$. The parietal region of the order of $40 \mathrm{~mm}$ (i.e., approximately 400 wall units, with a boundary layer thickness of about $300 \mathrm{~mm}$ ) provided a very good spatial resolution in the velocity measurements.

\subsection{Tomo-PIV Setup}

Figure 4.3 depicts the tomographic PIV arrangement, which was composed of 6 high-speed CMOS cameras recording a spanwise-wall-parallel volume and a Quantronix Nd:YLF laser $(2 \times 30 \mathrm{~mJ}$ at $1 \mathrm{kHz})$. The following cameras have been used: 3 Phantom V9.0 (1600x1200 pixels at 1000 fps with pixel size of $\left.11.5 \times 11.5 \mu \mathrm{m}^{2}\right)$, from LML, 1 Phantom V10 (2400x1800 pixels at $480 \mathrm{fps})$, from CORIA, and 2 Photron Fastcam APX (1024x1024 pixels at 2000 fps with pixel size of $17 \times 17 \mu \mathrm{m}^{2}$ ), provided by Pprime. In order to reach a recording rate of 2000 images per second, the region of interest of the Phantom CMOS cameras was reduced to $944 \mathrm{x} 944$ pixels. The 6 cameras were mounted on Scheimpflug adaptors ([Prasad, 2000]) designed by LML, which allowed precise adjustments of the position and the angle. They were positioned under the wind tunnel in a forward scattering configuration in a tilt angle of about $45^{\circ}$. The Phantom cameras had the sensor rotated of $45^{\circ}$ on the adapter in order to maximize the size of investigation volume. The Phantom cameras were set 
Table 4.1: Camera parameters for the boundary-layer tomo-PIV experiment.

\begin{tabular}{cccccc}
\hline camera & camera type & lens $(\mathrm{mm})$ & $f \#$ & $\theta_{z}(\mathrm{deg})$ & $\theta_{y}(\mathrm{deg})$ \\
\hline 1 & Phantom V9.0 & 200 & 5.6 & -35 & 35 \\
2 & Phantom V9.0 & 200 & 5.6 & -145 & 145 \\
3 & Phantom V9.0 & 200 & 5.6 & -145 & -145 \\
4 & Phantom V10 & 200 & 5.6 & -35 & -35 \\
5 & Photron Fastcam & $105(2 \mathrm{x})^{1}$ & 5.6 & -135 & 180 \\
6 & Photron Fastcam & $105(2 \mathrm{x})^{1}$ & 5.6 & -45 & 0 \\
\hline
\end{tabular}

${ }^{1}$ 105-mm lens equipped with a doubler

Table 4.2: Image parameters for the boundary-layer tomo-PIV experiment.

\begin{tabular}{cccccc}
\hline camera & bits & image size $($ pixels $)$ & pixel $(\mu \mathrm{m})$ & $M$ & cal $($ pixel $/ \mathrm{mm})$ \\
\hline 1 & 8 & $944 \times 944$ & 11.5 & 0.19 & 16.5 \\
2 & 8 & $944 \times 944$ & 11.5 & 0.19 & 16.3 \\
3 & 8 & $944 \times 944$ & 11.5 & 0.19 & 16.3 \\
4 & 8 & $944 \times 944$ & 11.5 & 0.19 & 16.2 \\
5 & 8 & $1024 \times 1024$ & 17.0 & 0.34 & 18.0 \\
6 & 8 & $1024 \times 1024$ & 17.0 & 0.34 & 17.8 \\
\hline
\end{tabular}

with micro Nikkor 200-mm lenses at f\# 5.6, while the Photron cameras were set with micro Nikkor 105-mm lens at f\# 5.6 with a doubler. The laser light sheet coming from the top of the wind tunnel passed through the transparent walls to form a volume normal to the flow direction (figure 4.5). Its thickness was limited to $5 \mathrm{~mm}$ by a slit composed of two blades attached to translation stages that accurately control its positioning and the light sheet thickness as is shown in figure 4.2. The overlapping field of view between all cameras produced an investigation volume of the size of $5 \times 45 \times 45 \mathrm{~mm}^{3}$ (i.e., 40x360x360 wall units). A summary of the cameras parameters is presented in tables 4.1 and 4.2, where $\mathrm{cal}$ is the conversion between image and laboratory dimensions, calculated by the calibration-target images, and the magnification is the ratio between imaged and real objects computed by $M=$ cal $\triangle_{\text {pixel }}$.

Some difficulties, inherent to the tomo-PIV were encountered in the accomplishment of this experiment since this technique requires high intensity illumination and spatial resolution of the image system. In addition, the imaging system requires a finite depth of focus corresponding to the thickness of the illuminated region ([Schröder et al., 2008a]). The last requirement is accomplished by reducing the optical aperture of the objectives, resulting in a further decrease of the particle intensity, which is even more problematic for air flows, where the particles are in general smaller than the particles used for water flows ([Novara, 2013]). In order to overcome these difficulties, and 


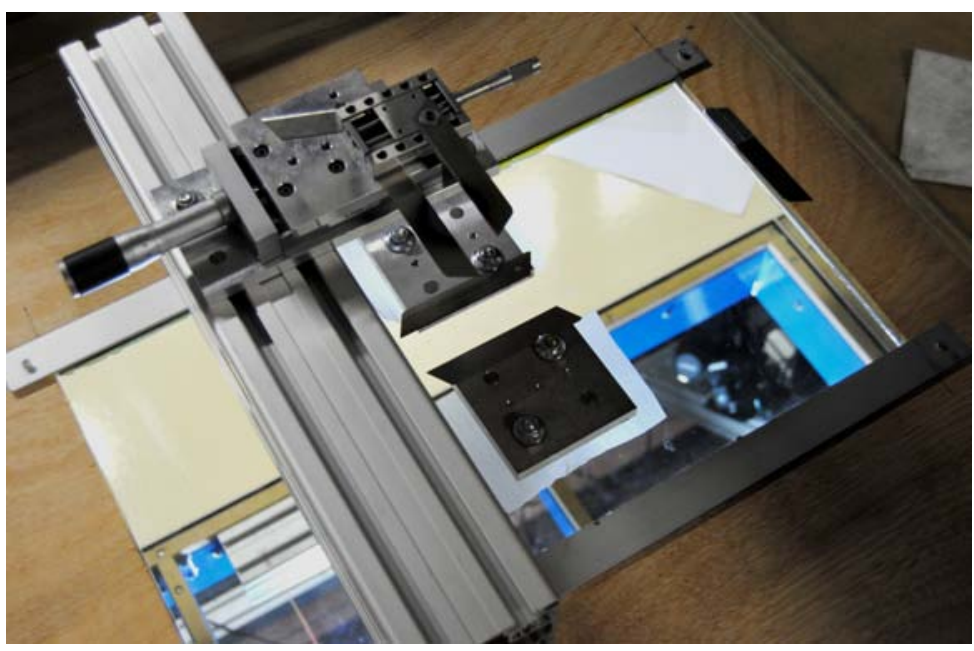

Figure 4.2: Blades fixed in a translation stage to compose the knife-edge filter.

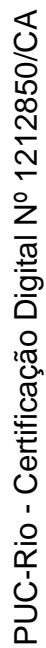
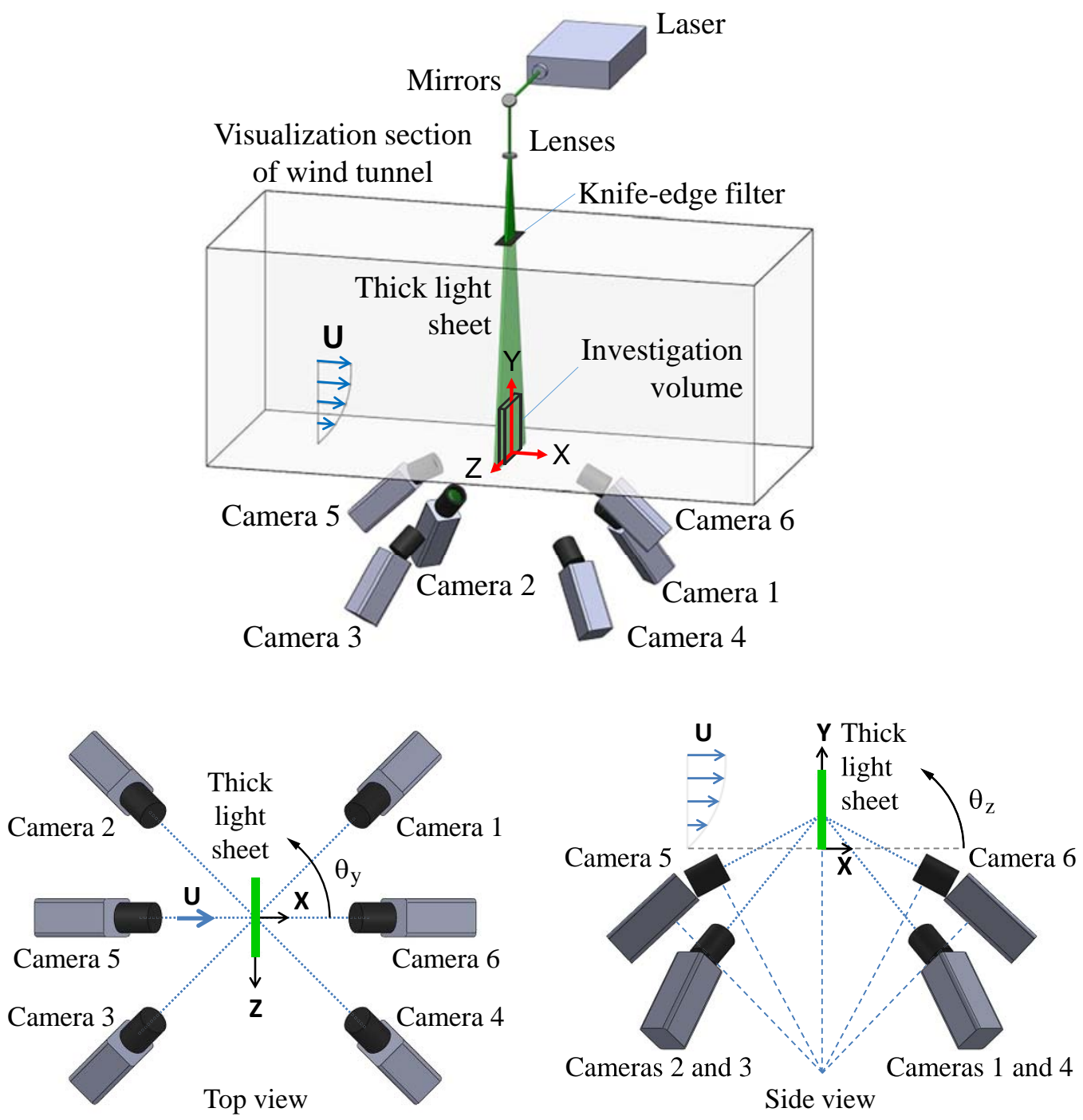

Figure 4.3: Sketch of the boundary-layer experimental setup. 


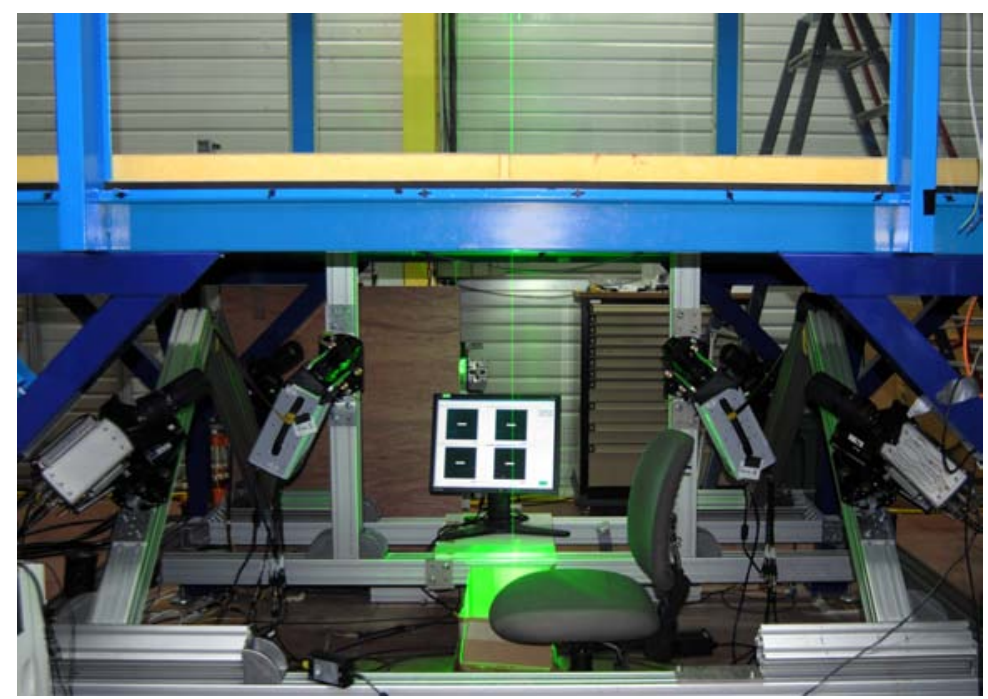

(a)

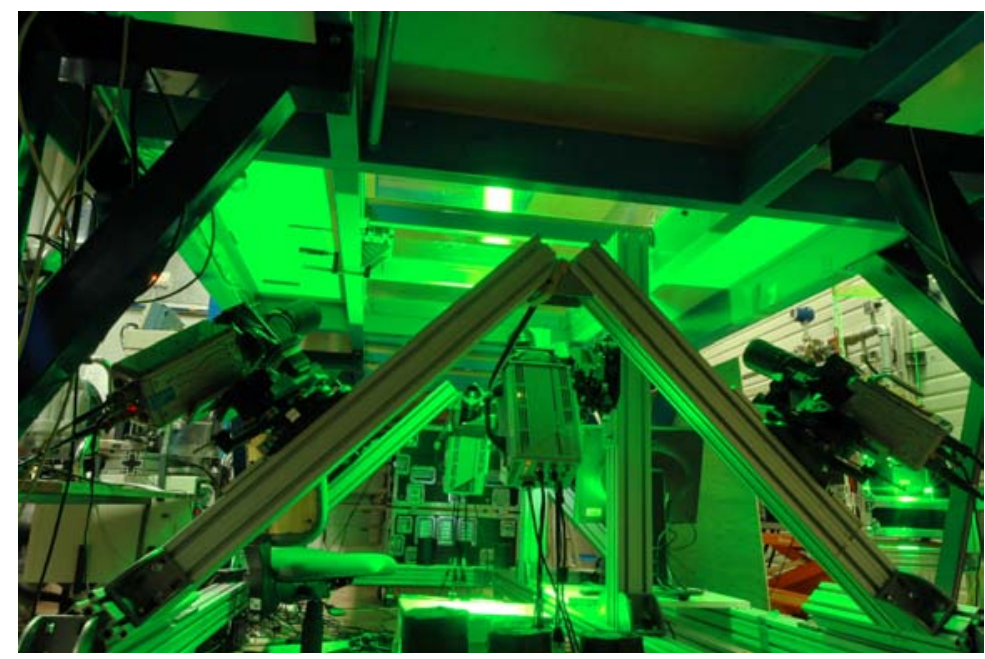

(b)

Figure 4.4: Pictures of the (a) side and (b) front views of the boundary-layer experimental setup.

to obtain accurate results, the thickness of the investigation volume has been limited to $5 \mathrm{~mm}$.

The whole flow was seeded with Poly-Ethylene Glycol smoke, which generates particles with sizes of the order of $d_{p}=1 \mu \mathrm{m}$. The mean particle concentration was between 0.01 and $0.06 \mathrm{ppp}$. The tomo-PIV images were recorded at $1 \mathrm{kHz}$ with a time delay of $300 \mu \mathrm{s}$ (corresponding to a maximum particle displacement of 12 voxels). The data acquisition was synchronized by a BNC pulse-delay generator (Berkeley Nucleonics Corporation, model 555). The grey level, not including reflections at the wall, ranged from 0 to 155 in the 8-bit images and the particle-image size was about 1 to 2 pixels. That value agrees with the estimation of the particle diameter in the image $d_{\tau}^{*}=1.3$ pixel given by eq. (3.2.2). For the experimental parameter values (i.e., $M$ given 


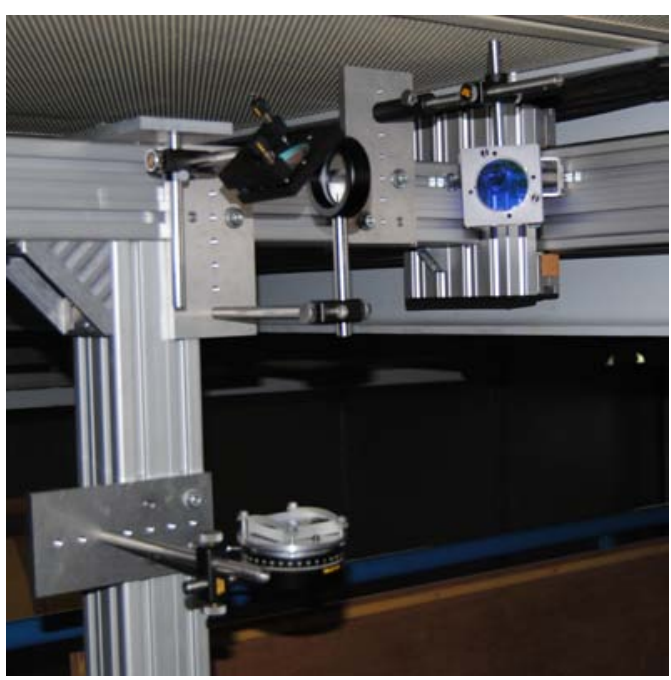

(a)

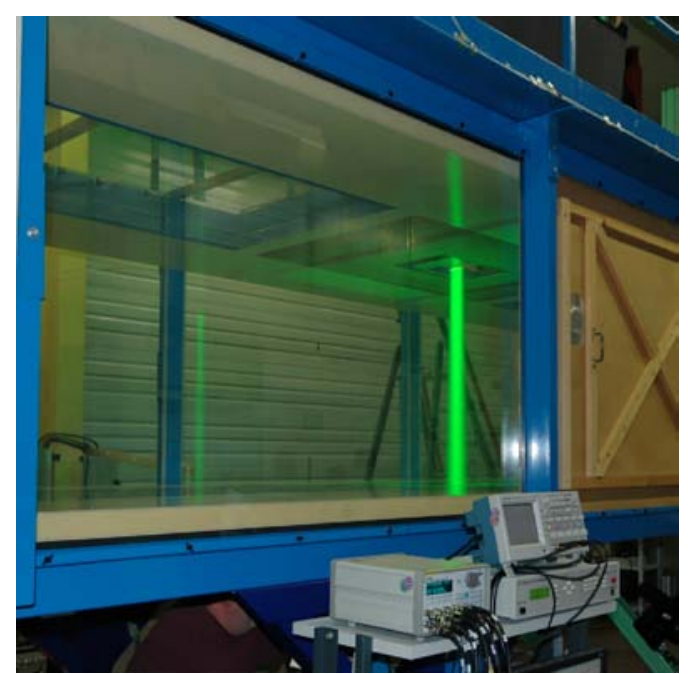

(b)

Figure 4.5: (a) Detail of the optical path of the laser light before the knife-edge filter. The light reflected in guiding mirrors, pass trough a diverging lens and a cylindrical lens. (b) Laser light sheet coming from the top of the wind tunnel. The system synchronizer, step-motor control of the calibration target and the multimeter are also visible in this picture.

by table $4.2, f \#=5.6$, and $\lambda=527 \mathrm{~nm}), d_{\text {diff }}$ was equal to $9.4 \mu \mathrm{m}$, so $d_{\tau}$ was approximately independent of the particle size for particle diameters lower than about $10 \mu \mathrm{m}$.

A total of 10 experimental runs of 1725 tomo-PIV fields were recorded in order to provide statistical results on the coherent organization of the flow structures. Its particle densities, which were computed by a local maxima, are exposed in the table 4.3.

Figure 4.6 shows samples of particle image concentration of 0.010 (run 14), 0.029 (run 13), 0.057 (run 17) and 0.070 ppp (run 16) recorded by camera 1 . The images of low particle concentration were used to improve the calibration, while images with intermediate amount of particles were used in the statistical analysis of this work. The high concentration particle images were not employed in this analysis due to the amount of ghost particles in the reconstructed volume as will be discussed in chapter 5. Problems as laser light reflections (bright agglomerate of pixels along a diagonal starting on the bottom-left corner) and non-uniform illumination are present and were corrected by image pre-processing that will be presented also in chapter 5 .

\subsection{Calibration}

In the boundary-layer experiment the calibration of the cameras imaging a volume was done by means of a home-made $2 \mathrm{D}$ target placed inside the wind 
Table 4.3: Average particle density per pixel for each experimental run.

\begin{tabular}{cc}
\hline Run & Particle density $(p p p)$ \\
\hline 10 & 0.060 \\
11 & 0.057 \\
12 & 0.025 \\
13 & 0.029 \\
14 & 0.010 \\
15 & 0.064 \\
16 & 0.070 \\
17 & 0.057 \\
18 & 0.039 \\
19 & 0.011 \\
\hline
\end{tabular}

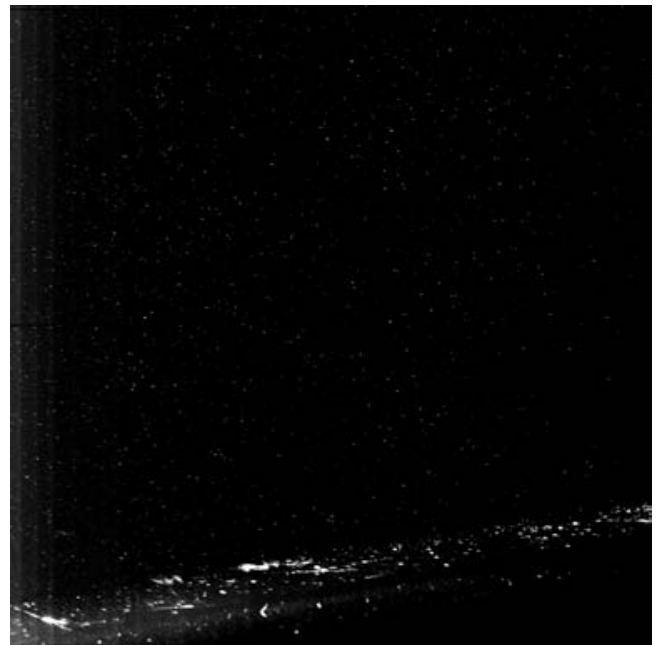

(a)

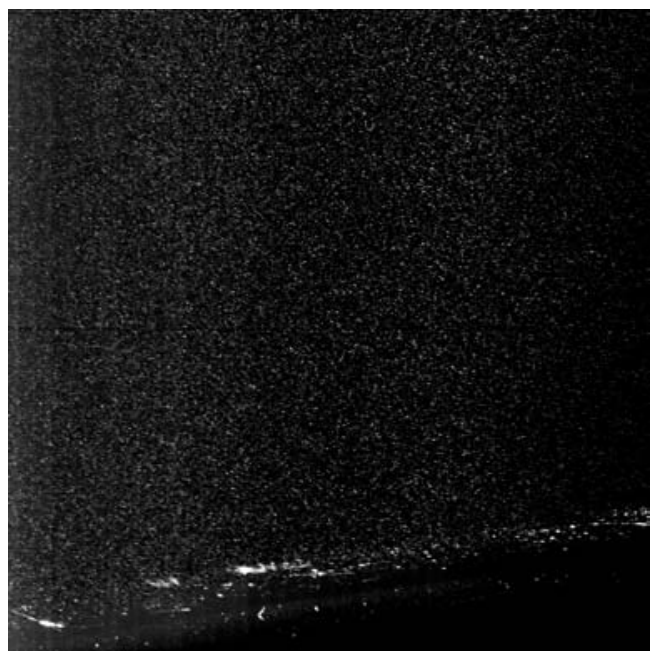

(c)

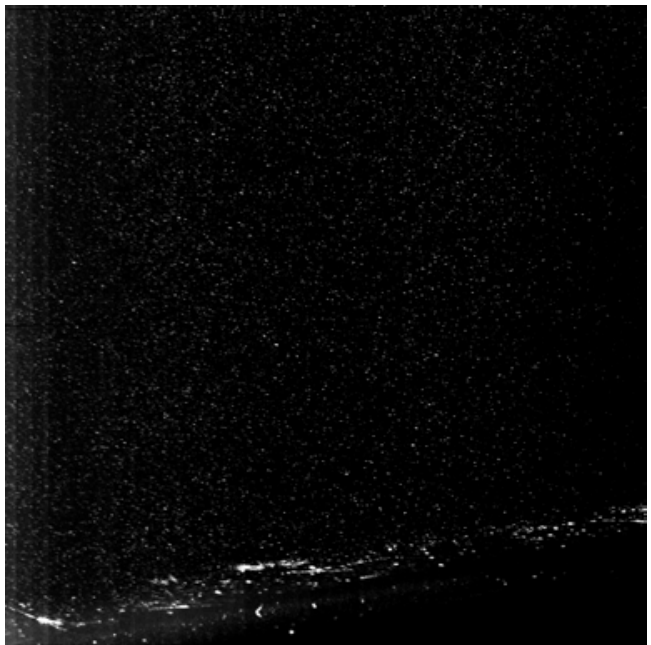

(b)

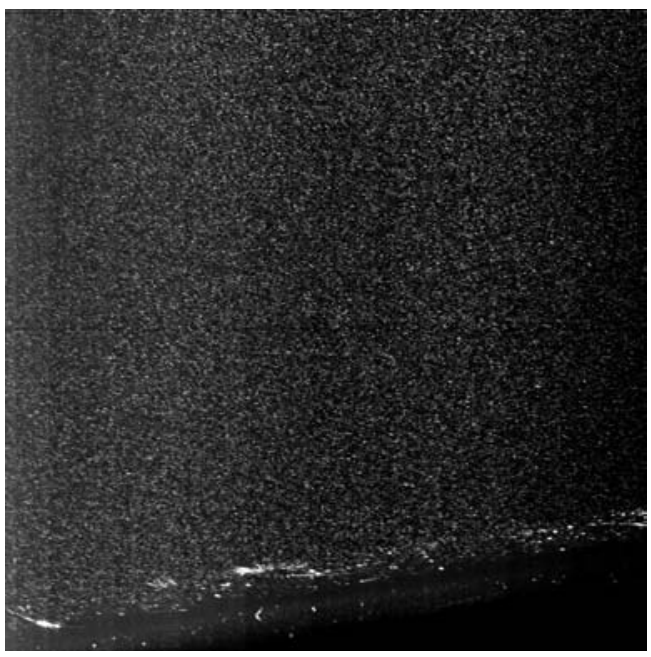

(d)

Figure 4.6: Samples of particle image concentration of (a) 0.010, (b) 0.029, (c) 0.057 and (d) $0.070 \mathrm{ppp}$ recorded by camera 1 . The brightness and contrast of the images were adjusted to enhance visualization. 
tunnel before each experimental run. The calibration target was transparent in order to allow for the visualization of the markers from both sides. It was fabricated from a transparent plastic sheet with crosses pattern printed on it. The plastic sheet was sandwiched between two 2-mm-thick glass plates in order to provide mechanical rigidity. Initially aligned with the laser sheet at $z^{*}=0$ (see figure $4.7(\mathrm{a})$ ), the target was translated in the out-of-plane direction from $z^{*}=-5 \mathrm{~mm}$ up to $z^{*}=5 \mathrm{~mm}$ with steps of $0.50 \mathrm{~mm}$ using a micrometer with $5 \mu \mathrm{m}$ precision. The target was illuminated by light sources from both sides (see figure 4.7(b)) and, at each position, its projection images in the cameras were recorded. The target had a distance of $5 \mathrm{~mm}$ between 2 consecutive crosses in both $x^{*}$ and $y^{*}$ directions. Figure 4.8 presents the calibration images at $z^{*}=0$ for the 6 cameras. The $u-v$ image coordinate system of each camera, the $x^{*}-y^{*}-z^{*}$ world coordinate system adopted and the $x-y-z$ wind-tunnel system are also sketched in this figure.

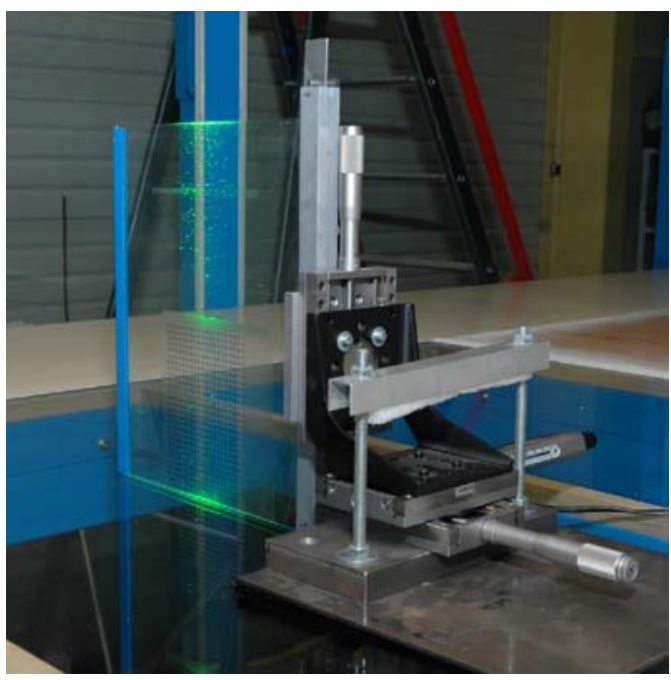

(a)

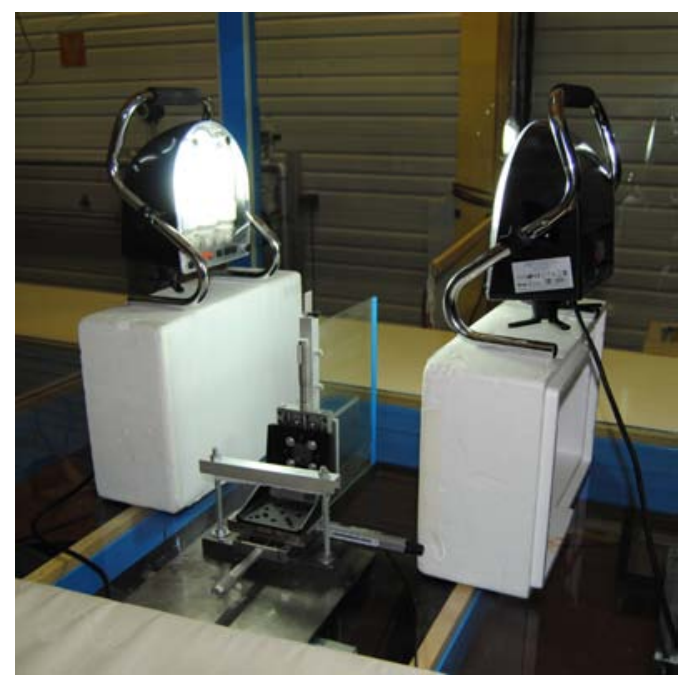

(b)

Figure 4.7: Calibration target fixed at the translation unit in order to record multiple planes. Detail of (a) the target plane at the position $z^{*}=0$ at the middle of the laser volume and (b) the light source arrangement during the calibration recordings.

The calibration procedure that is explained as follows was produced by means of the tomo-PIV software developed together by Pprime, LML and Coria laboratories ([Thomas et al., 2014, Kähler et al., 2016]).

The markers of the calibration target, in that case black crosses pattern on a white background, were automatically located by a centroid method. Employing these locations, the calibration of each camera was performed, mapping the world points onto the camera images. The world coordinate system was chosen far away from the borders of the image, avoiding possible 

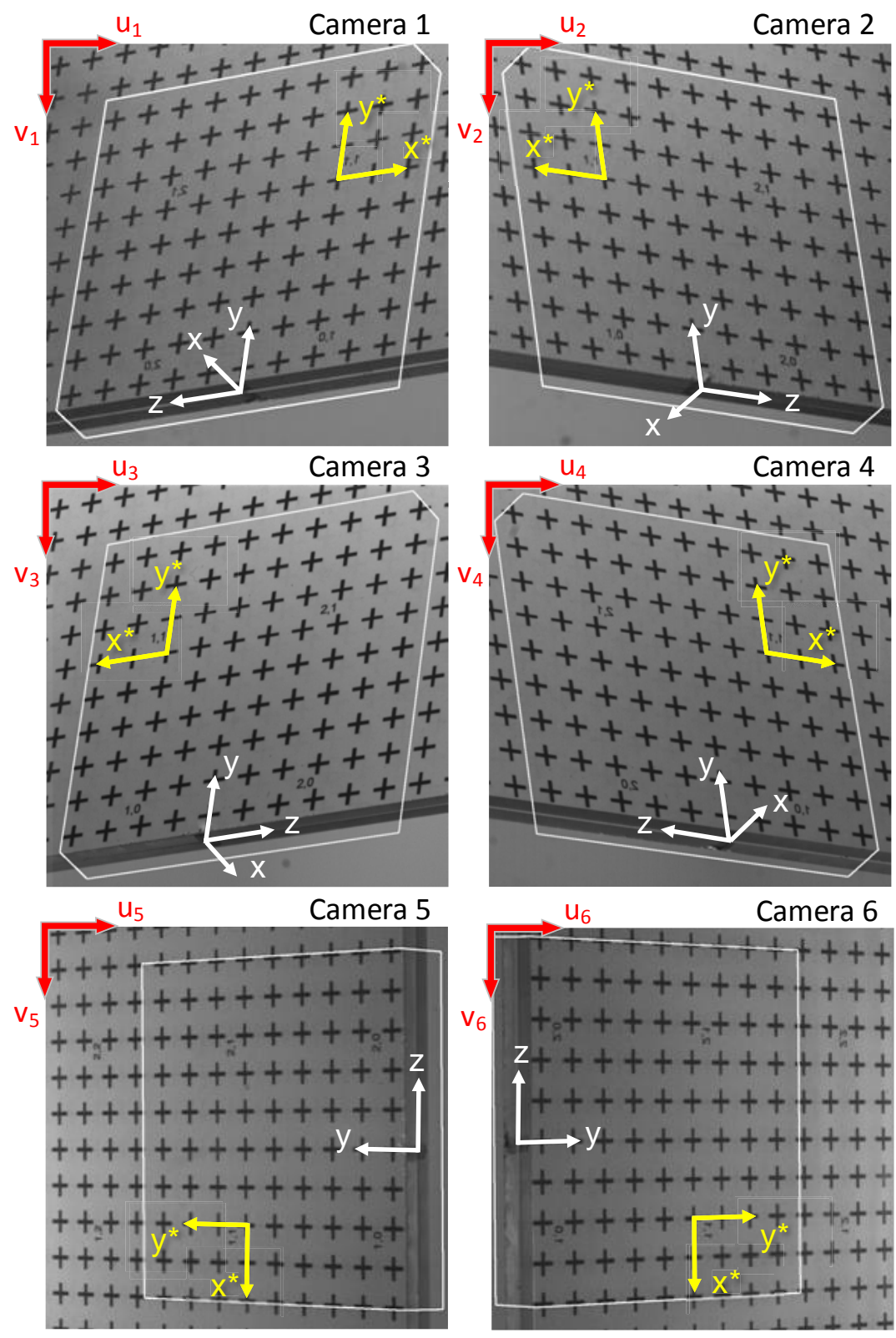

Figure 4.8: Target image for each camera. The images show the projection of the reconstructed volume limits, the image coordinate system (in red), the world coordinate system (in yellow) and the wind-tunnel coordinate system (in white). In order to enhance the visualization the third axis from the world and wind-tunnel systems can be suppressed sometimes. 
aberrations, and close to an identification number, facilitating the visual location in all camera views (figure 4.8).

The pinhole model, given by eq. (3.2.9), was tested to calibrate camera 1 employing 21 planes (i.e., $-5,-4.5, \ldots, 5 \mathrm{~mm}$ ), 13 planes (i.e., $-3,-3.5, \ldots, 3 \mathrm{~mm}$ ) and 7 planes (i.e., $-3,-2, \ldots, 3 \mathrm{~mm}$ ) leading to initial projection errors of 0.25 to 0.29 . Tests were also made using the Soloff's model, eq. (3.2.10), producing slightly smaller errors. Since the thickness of the laser sheet was about $5 \mathrm{~mm}$ and the images did not have significant aberrations, the pinhole model with 7 planes was chosen due to its physical approach and lower errors compared to the use of larger amount of calibration planes.

The tomographic reconstruction requires that each voxel position in space is mapped onto a camera pixel position with an error lower than 0.1 pixel ([Wieneke, 2008]). So, in order to reduce the calibration errors, the mapping functions were corrected by an iterative self-calibration procedure based on the particle images (section 3.2.4).

Due to the way the target was manufactured, the refraction phenomena of light in the glass plates of 2-mm thick used in each side of it created significant triangulation errors computed by eq. (3.2.11). After the removal of the target, the error between the apparent position of the crosses along $z^{*}$ from the cameras placed downstream (i.e., 1, 4 and 6) and the ones upstream was greater than $2 \mathrm{~mm}$. So a high number of spurious particle matchings appeared and spread the real disparity peak, making the correction of the mapping function by the standard self-calibration method ([Wieneke, 2008]) very difficult. The increase of search region around the "epipolar line" ([Wieneke, 2008]) did not ameliorate the statistics of the disparity peaks. The use of the 4-camera limited self-calibration ([Discetti and Astarita, 2013]) was also tried and did not solve that problem.

To circumvent this refraction issue, the target planes along $z^{*}$ were artificially translated on $1.1 \mathrm{~mm}$ for the downstream cameras and on $-1.1 \mathrm{~mm}$ for the upstream ones. Theses distances were calculated by the Snell's equation according to eq. (4.3.1). The optical phenomena and its solution are depicted in figure 4.9. Considering the target marker as a light source point with an incident angle $\theta_{2}$ of $28.13^{\circ}$ (blue line), the glass refraction index $n_{2}$ of about 1.5 and the air refraction index $n_{1}$ equal to 1 , that lead to a refraction angle $\theta_{1}$ of $45^{\circ}$ (i.e., the camera line-of-sight). Then, a second incident ray from the same source point was drawn with an incident angle slightly different like $28^{\circ}$ (grey line). The prolongation of these two refracted rays showed an estimation of the virtual point position seen by the camera. 


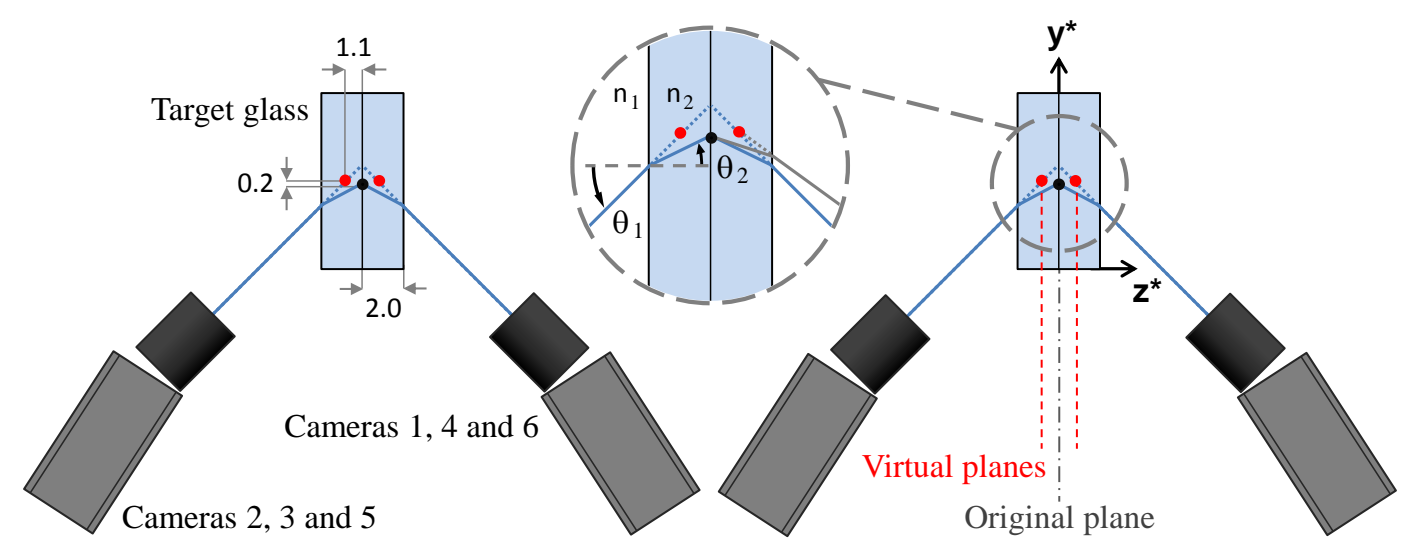

(a)

(b)

Figure 4.9: Refraction phenomena in the target glass showing (a) the distance (in $\mathrm{mm}$ ) between the real target marker (black point) to the virtual ones (red points) and (b) the virtual planes adopted (dashed red lines) instead of the real target plane (dash-dotted grey line).

$$
\frac{\sin \theta_{1}}{\sin \theta_{2}}=\frac{n_{2}}{n_{1}}
$$

The first camera calibrations were made with these virtual planes. The displacement of the planes do not need to be exact, they were just a first guess to reduce the disparity errors. The difference between the real location along $y$ was the same for all cameras and introduced just a small offset that was corrected during the post-processing. The new self-calibration method using the translated planes for compensation of multimedia geometry will be referred in the text from now on by the prefix translated-plane.

In order to increase the signal to noise on the disparity maps, particle images of the low-concentration experiment (run 14) with a seeding density of $0.01 p p p$ were used for the self- calibration. The self-calibration procedure was computed using a interrogation volume size of $3.8 \times 15 \times 15 \mathrm{~mm}$ (i.e., $x, y, z$ of $53 \times 210 \times 210$ voxels) with $75 \%$ of overlapping in a reconstruction volume of size $86 \times 651 \times 631$ voxels (which gave a disparity maps of the size 3x9x8). The selfcalibration parameters were: a search region of 3 pixels, 0.05 pixel of precision in the disparity map, a blob of 7-pixels size and a maximum number of 5000 particles in 50 time-independent particle images. As pre-processing, the images were filtered by a local-mean-background subtraction with a window size of $21 \times 21$ pixels. The outcome 3D-2C disparity vector field was used to correct the camera model. With these new mapping functions of each camera, a new selfcalibration could be performed. The disparity map of camera 1 at the middle of the laser sheet for the first iteration of the translated-plane self-calibration method is shown in figure 4.10. At total, 3 iterations of the procedure were used. 


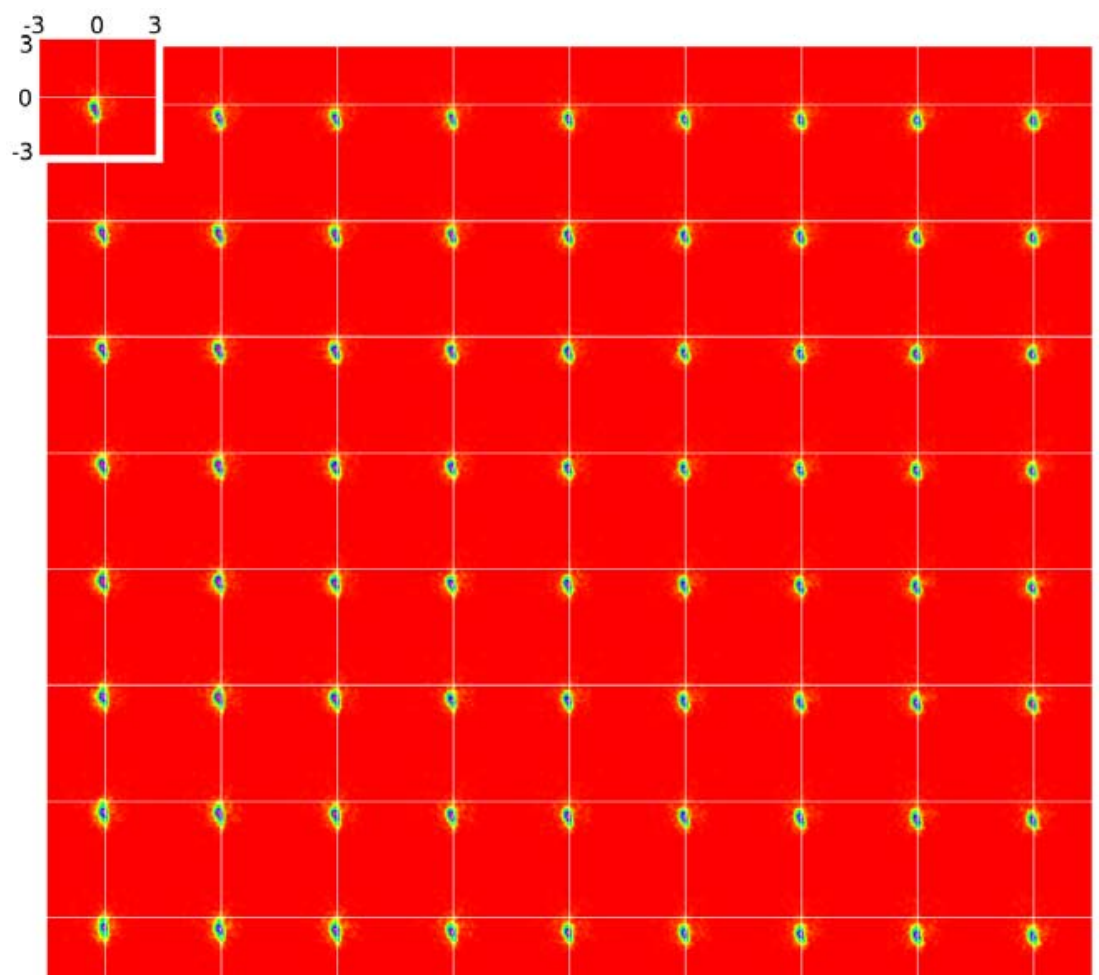

Figure 4.10: 2D disparity map of camera 1 at the middle of the laser sheet for the translated-plane self-calibration method with a search region of 3 pixels.

The disparity maps in the region evidenced in figure 4.10 of the first self-calibration iteration using the standard method, the 4-camera limited algorithm and the translated procedure are amplified in figure 4.11. For the standard and the 4-camera limited methods using the original planes, a search region around the epipolar line of 6 and 9 pixels were also tried decreasing the number of maximum particles to 1000 and increasing the number of particle images to 500. These additional self-calibrations did not yield good results, corroborating the importance of the use of the translated-plane method in tomo-PIV experiments employing glass target visualized from both sides.

The correction of the mapping function by the translated-plane selfcalibration method can be visualized from the decreasing of the errors along each step in the table 4.4. After the refraction correction, and 3 iterations of the self-calibration procedure explained before, the errors were significantly reduced as shown in the disparity maps of figure 4.12. The final pinhole mapping functions for the experimental run 14 had projection errors of about 0.01 pixel and triangulation errors lower than 0.05 pixel in the entire domain. More self-calibration iterations did not improve the results.

Unfortunately this corrected mapping function for run 14 was not valid for the other experiment runs due to minor changes in the camera positions and laser illumination. The triangulation errors in other runs using these corrected 

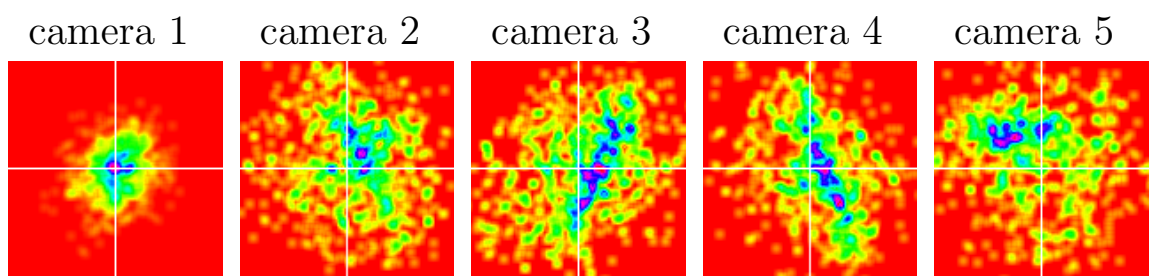

camera 6

standard self-calibration
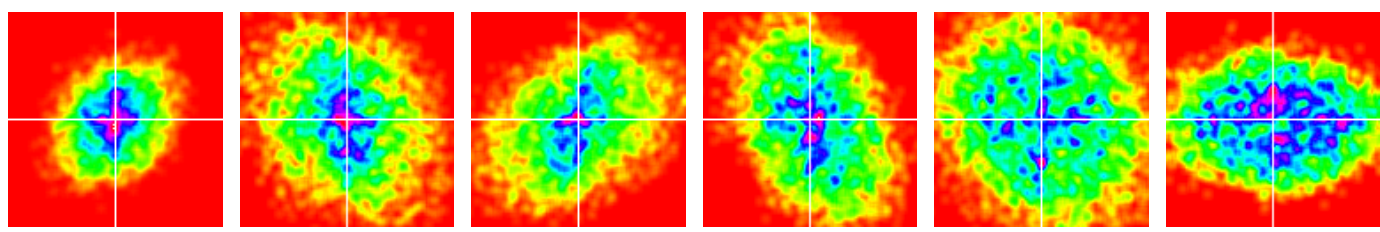

4-camera limited self-calibration
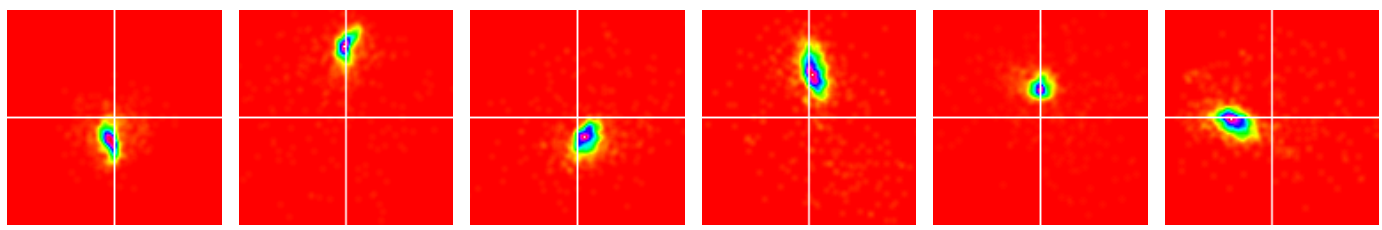

translated-plane self-calibration

Figure 4.11: Amplified views of the upper-left corner of disparity maps for search region of 3 pixels: standard method, 4-camera limited algorithm and translated-plane self-calibration procedure.

mapping functions were higher than the tolerable 0.4 pixel (e.g., run 17 gave a maximum triangulation error of 0.6 pixel).

Applying the self-calibration directly to the high particle concentration runs, using a threshold to decrease the amount of particles, did not yield good results. In these cases (e.g., particle concentration of $0.03 \mathrm{ppv}$, volume thickness of 80 voxels and camera tilt angle of $45^{\circ}$ ), there was a high probability to find more than 3 particles along the same line-of-sight.

To solve this problem, the final pinhole mapping functions for run 14 was used as a first guess of the camera calibration for the other runs that was then re-corrected by four more self-calibration steps using a maximum number of 3500 particles in 150 time-independent images. Increasing the amount of iteration did not ameliorate the results.

The final calibration errors for each camera in each experimental run can be found in the table 4.5. In runs 15 and 16, with higher particle densities of about $0.07 p p p$, the self-calibration presented some difficulties that resulted in higher triangulation errors. The final pinhole mapping functions, which were different in each run, had projection errors of about 0.02 pixel and triangulation errors lower than 0.1 to 0.25 pixel in the entire domain, which is enough to produce quality reconstruction volumes ([Wieneke, 2008]).

To translate the world coordinate system to the wind-tunnel coordinate 
camera 1 camera 2 camera 3 camera 4 camera 5 camera 6
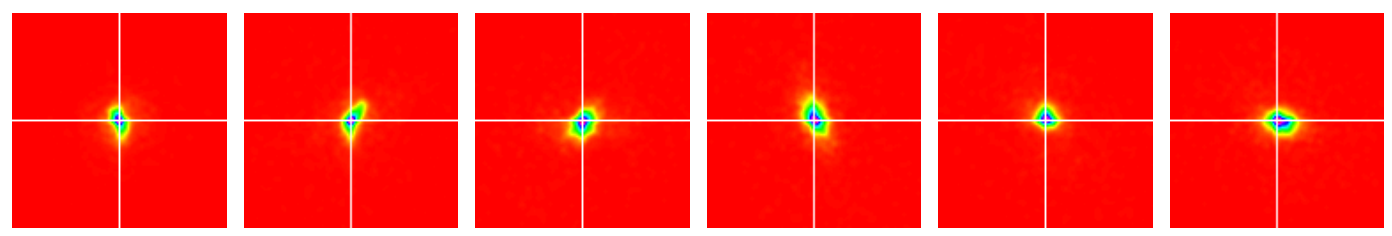

Figure 4.12: Amplified views of the upper-left corner of disparity maps for search region of 3 pixels after the translated-plane self-calibration correction.

Table 4.4: Evolution of the projection and the triangulation errors of each camera during the translated-plane self-calibration corrections for run 14 (in pixel).

\begin{tabular}{|c|c|c|c|c|c|c|}
\hline & \multicolumn{3}{|c|}{ projection error } & \multicolumn{3}{|c|}{ triangulation error } \\
\hline & $x_{\mathrm{rms}}$ & $y_{\mathrm{rms}}$ & $\max$ & $x_{\mathrm{rms}}$ & $y_{\mathrm{rms}}$ & $\max$ \\
\hline \multicolumn{7}{|l|}{ step 1} \\
\hline camera 1 & 0.27 & 0.29 & 1.02 & 0.82 & 0.10 & 1.10 \\
\hline camera 2 & 0.20 & 0.32 & 0.81 & 2.19 & 0.03 & 2.30 \\
\hline camera 3 & 0.26 & 0.23 & 0.83 & 0.60 & 0.22 & 0.80 \\
\hline camera 4 & 0.21 & 0.31 & 0.91 & 1.44 & 0.03 & 1.65 \\
\hline camera 5 & 0.27 & 0.36 & 1.01 & 0.80 & 0.05 & 0.90 \\
\hline camera 6 & 0.29 & 0.41 & 1.18 & 0.12 & 1.22 & 1.42 \\
\hline \multicolumn{7}{|l|}{ step 2} \\
\hline camera 1 & 0.04 & 0.02 & 0.10 & 0.01 & 0.03 & 0.07 \\
\hline camera 2 & 0.02 & 0.01 & 0.05 & 0.00 & 0.02 & 0.03 \\
\hline camera 3 & 0.02 & 0.02 & 0.08 & 0.03 & 0.02 & 0.05 \\
\hline camera 4 & 0.04 & 0.01 & 0.11 & 0.11 & 0.02 & 0.30 \\
\hline camera 5 & 0.01 & 0.01 & 0.04 & 0.03 & 0.03 & 0.08 \\
\hline camera 6 & 0.03 & 0.03 & 0.10 & 0.05 & 0.02 & 0.20 \\
\hline \multicolumn{7}{|l|}{ step 3} \\
\hline camera 1 & 0.00 & 0.00 & 0.02 & 0.01 & 0.03 & 0.07 \\
\hline camera 2 & 0.00 & 0.01 & 0.02 & 0.00 & 0.02 & 0.03 \\
\hline camera 3 & 0.01 & 0.01 & 0.03 & 0.03 & 0.02 & 0.05 \\
\hline camera 4 & 0.03 & 0.01 & 0.06 & 0.05 & 0.02 & 0.20 \\
\hline camera 5 & 0.01 & 0.01 & 0.02 & 0.03 & 0.03 & 0.07 \\
\hline camera 6 & 0.01 & 0.01 & 0.03 & 0.04 & 0.02 & 0.10 \\
\hline \multicolumn{7}{|l|}{ step 4} \\
\hline camera 1 & 0.00 & 0.00 & 0.01 & 0.00 & 0.03 & 0.07 \\
\hline camera 2 & 0.00 & 0.00 & 0.01 & 0.00 & 0.02 & 0.03 \\
\hline camera 3 & 0.01 & 0.01 & 0.03 & 0.03 & 0.02 & 0.05 \\
\hline camera 4 & 0.01 & 0.01 & 0.03 & 0.04 & 0.02 & 0.10 \\
\hline camera 5 & 0.01 & 0.01 & 0.02 & 0.03 & 0.02 & 0.07 \\
\hline camera 6 & 0.01 & 0.01 & 0.03 & 0.04 & 0.02 & 0.10 \\
\hline
\end{tabular}


Table 4.5: Projection and the triangulation errors of the camera calibrations for each run (in pixel).

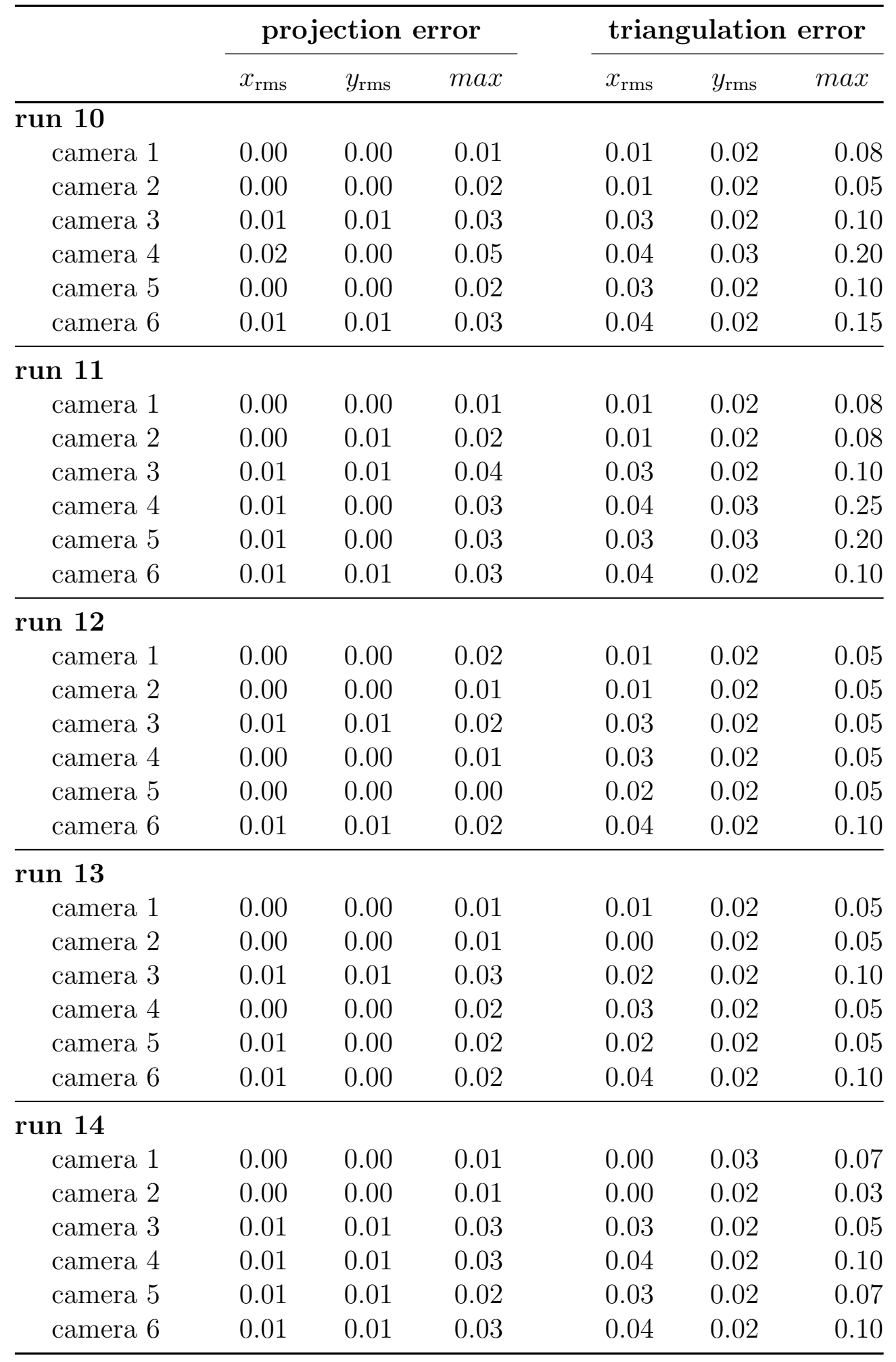




\begin{tabular}{|c|c|c|c|c|c|c|}
\hline & \multicolumn{3}{|c|}{ projection error } & \multicolumn{3}{|c|}{ triangulation error } \\
\hline & $x_{\mathrm{rms}}$ & $y_{\mathrm{rms}}$ & $\max$ & $x_{\mathrm{rms}}$ & $y_{\mathrm{rms}}$ & $\max$ \\
\hline \multicolumn{7}{|l|}{ run 15} \\
\hline camera 1 & 0.01 & 0.00 & 0.02 & 0.02 & 0.02 & 0.07 \\
\hline camera 2 & 0.01 & 0.01 & 0.04 & 0.03 & 0.02 & 0.15 \\
\hline camera 3 & 0.00 & 0.01 & 0.02 & 0.02 & 0.03 & 0.25 \\
\hline camera 4 & 0.00 & 0.00 & 0.02 & 0.02 & 0.03 & 0.20 \\
\hline camera 5 & 0.01 & 0.01 & 0.03 & 0.01 & 0.03 & 0.08 \\
\hline camera 6 & 0.01 & 0.01 & 0.03 & 0.03 & 0.03 & 0.20 \\
\hline \multicolumn{7}{|l|}{ run 16} \\
\hline camera 1 & 0.02 & 0.02 & 0.06 & 0.04 & 0.05 & 0.25 \\
\hline camera 2 & 0.01 & 0.01 & 0.02 & 0.03 & 0.03 & 0.20 \\
\hline camera 3 & 0.02 & 0.01 & 0.05 & 0.05 & 0.04 & 0.25 \\
\hline camera 4 & 0.02 & 0.01 & 0.09 & 0.05 & 0.04 & 0.20 \\
\hline camera 5 & 0.01 & 0.01 & 0.05 & 0.03 & 0.06 & 0.22 \\
\hline camera 6 & 0.01 & 0.02 & 0.07 & 0.03 & 0.04 & 0.22 \\
\hline \multicolumn{7}{|l|}{ run 17} \\
\hline camera 1 & 0.01 & 0.01 & 0.03 & 0.02 & 0.02 & 0.07 \\
\hline camera 2 & 0.00 & 0.01 & 0.03 & 0.02 & 0.02 & 0.10 \\
\hline camera 3 & 0.01 & 0.01 & 0.03 & 0.02 & 0.03 & 0.07 \\
\hline camera 4 & 0.01 & 0.01 & 0.05 & 0.03 & 0.04 & 0.18 \\
\hline camera 5 & 0.01 & 0.01 & 0.03 & 0.03 & 0.04 & 0.18 \\
\hline camera 6 & 0.01 & 0.01 & 0.04 & 0.02 & 0.02 & 0.05 \\
\hline \multicolumn{7}{|l|}{ run 18} \\
\hline camera 1 & 0.01 & 0.00 & 0.02 & 0.02 & 0.02 & 0.05 \\
\hline camera 2 & 0.00 & 0.01 & 0.02 & 0.01 & 0.02 & 0.05 \\
\hline camera 3 & 0.01 & 0.01 & 0.03 & 0.02 & 0.02 & 0.07 \\
\hline camera 4 & 0.00 & 0.00 & 0.01 & 0.02 & 0.02 & 0.10 \\
\hline camera 5 & 0.00 & 0.01 & 0.02 & 0.02 & 0.02 & 0.05 \\
\hline camera 6 & 0.01 & 0.01 & 0.02 & 0.01 & 0.02 & 0.05 \\
\hline \multicolumn{7}{|l|}{ run 19} \\
\hline camera 1 & 0.00 & 0.00 & 0.02 & 0.00 & 0.02 & 0.07 \\
\hline camera 2 & 0.00 & 0.01 & 0.02 & 0.00 & 0.02 & 0.03 \\
\hline camera 3 & 0.00 & 0.01 & 0.03 & 0.02 & 0.03 & 0.07 \\
\hline camera 4 & 0.00 & 0.00 & 0.01 & 0.01 & 0.02 & 0.07 \\
\hline camera 5 & 0.00 & 0.00 & 0.01 & 0.01 & 0.02 & 0.05 \\
\hline camera 6 & 0.01 & 0.00 & 0.03 & 0.02 & 0.02 & 0.05 \\
\hline
\end{tabular}


system, the position of the tunnel bottom wall must be calculated. In order to infer the wall-normal position of the origin of the wind-tunnel coordinate system, the laser light reflections on the glass surface was used. The light reflections can be obtained by the time-history minimum intensity of the experimental run for each camera. The origin of the coordinate system can be obtained by projecting the investigation volume bottom wall onto the reflection images. The base of the investigation volume must contain the majority of the reflections, as displayed in figure 4.13. The offset of the wall-normal direction obtained has an accuracy better than 3 pixels (equivalent of $0.19 \mathrm{~mm}$ ) and should be fine tuned in the estimation of the friction velocity as will be described in chapter 8 . 
Camera 1

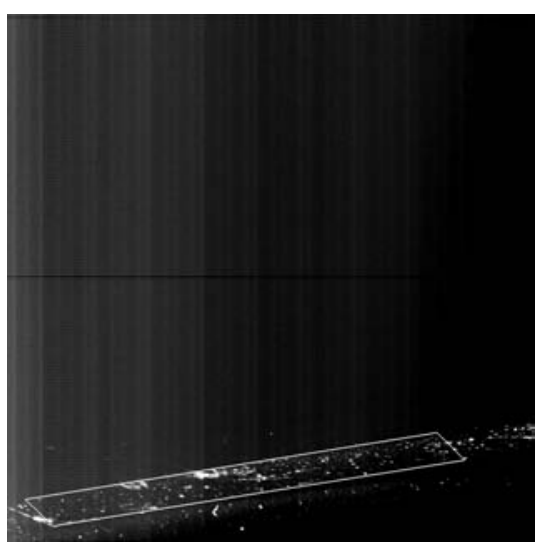

Camera 3

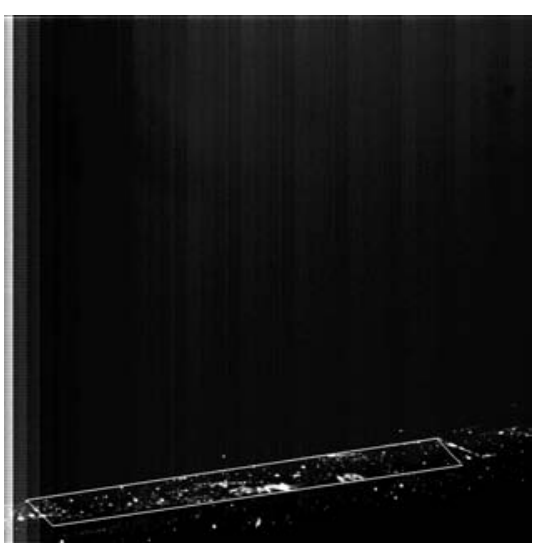

Camera 5

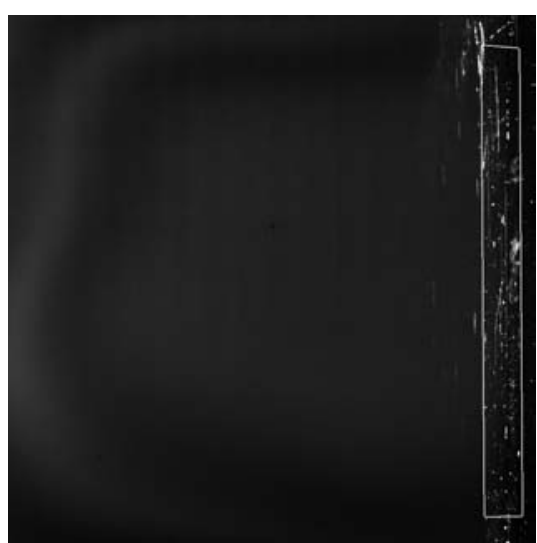

Camera 2

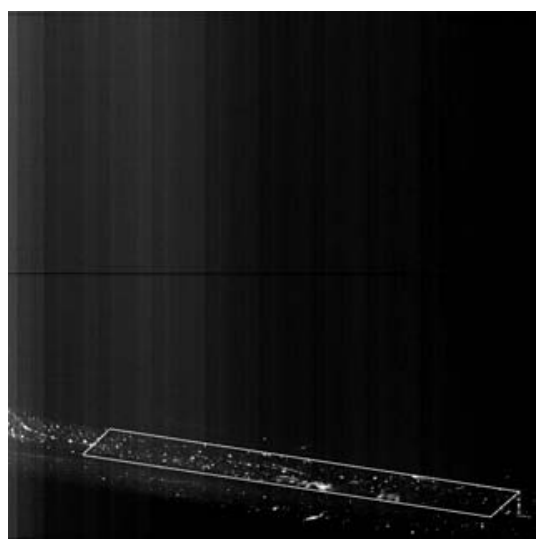

Camera 4

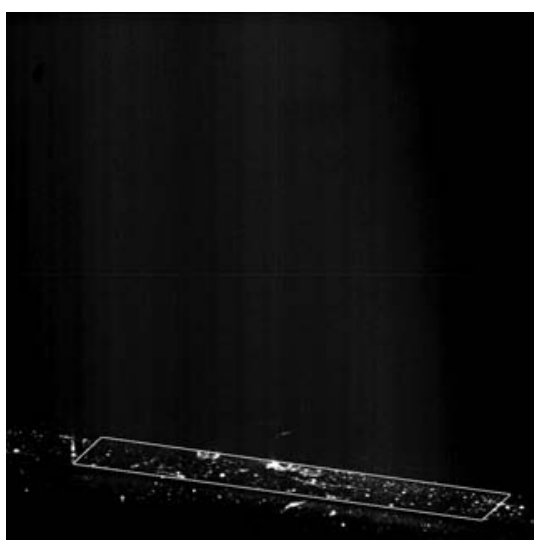

Camera 6

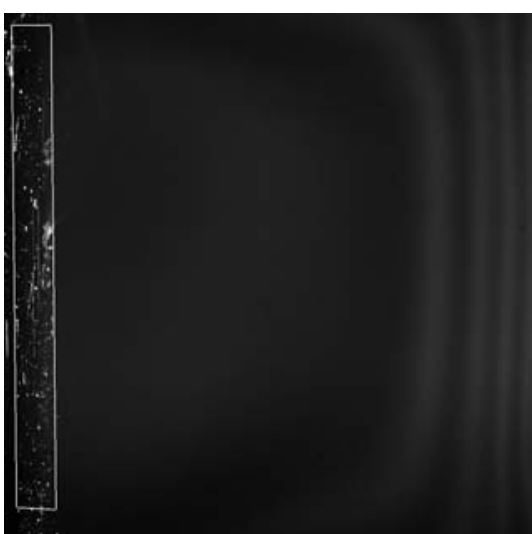

Figure 4.13: Contour of the base of investigation volume projected onto the camera images of light reflections. The brightness and contrast of the timehistory minimum intensity images were adjusted to enhance visualization. 


\section{5}

\section{Tomo-PIV Optimization Results}

The performance and accuracy of tomographic PIV technique is highly dependent on the parametric adjustment and the reconstruction algorithm used. Although synthetic data have been widely employed to optimize experiments, the resulting reconstructed volumes might not have optimal quality. The purpose of this chapter is to apply quality indicators to data samples in order to improve the quality of velocity results obtained by the tomo-PIV technique. Tomo-PIV data provided by a six-camera turbulent boundary-layer experiment were used to optimize the reconstruction algorithms according to the methodology described in section 3.3. Velocity statistics measurements obtained by optimized BIMART, SMART and MART algorithms were compared with hot-wire anemometer data and velocity measurement uncertainties were computed.

The present chapter produced the following publication:

Martins, F. J., Foucaut, J. M., Thomas, L., Azevedo, L. F., and Stanislas, M. (2015). Volume reconstruction optimization for tomo-PIV algorithms applied to experimental data. Measurement Science and Technology, 26(8), 085202.

\subsection{Volume Reconstruction Optimization}

The next sections will present optimization results for the tomo-PIV technique applied to the boundary-layer data obtained from the experiments described in the previous section. In order to facilitate the identification of the optimization phases of the tomo-PIV technique, the technique is divided into 6 phases, that are defined as follows.

Phase 1: image acquisition;

Phase 2: image pre-processing;

Phase 3: volume reconstruction;

Phase 4: pre-processing of reconstructed volumes;

Phase 5: cross-correlation of reconstructed volumes;

Phase 6: post-processing of calculated velocity fields. 
Results will be presented for the optimization of image pre-processing methods (Phase 2) applied to the acquired boundary-layer images, and to volume filtering methods applied to improve the performance of volume reconstruction (Phase 3). Although the optimization of other tomo-PIV parameters has been performed, it will not be presented here due to the specific nature of the reconstruction code employed. These optimization procedures are applied to small samples of the data set, so that the processing of the complete image data set can be done more efficiently and accurately. Some of the quality indicators described in the present paper will be used to guide the optimization process. In addition to the optimization results, the influence of the number of cameras and particle density on the reconstructed volumes will be addressed.

For all the results to be presented next, a 5-iteration standard implementation of the MART reconstruction algorithm was employed. In the algorithm used, the voxel weight function is computed by the intersection of a sphere and a cylinder ([Lamarche and Leroy, 1990]). In the present tomo-PIV data set, the use of the OTF ([Schanz et al., 2013a]) as the voxel weight function is not necessary because the images of the particles are well identifiable without suggestive aberrations (as astigmatism, for example). The algorithm initialization is implemented by a minimum line-of-sight method, based on the good results obtained in prior tests. The results were obtained employing a $C^{++}$ parallelized tomo-PIV software developed in a cooperation among the laboratories Pprime, from the Université de Poitiers, Coria, from the Université de Rouen, and LML, from the École Centrale de Lille.

\subsubsection{Image Pre-Processing}

Background reflections in tomo-PIV images are known to bias velocity measurements and their associate statistics. In order to improve the signal of particles in the original images, several pre-processing (Phase 2 of the tomo-PIV technique) methods were tested. Due to space limitations, only the results of six image pre-processing filters were selected for presentation that are described as follows.

(a) subMean: subtraction of a time-averaged image computed from the complete set of instantaneous images;

(b) subMedian: subtraction of a time-series median image from the instantaneous images;

(c) subMin: subtraction of a time-series minimum from the instantaneous images; 
(d) +localMin: combination of previous filters with a minimum intensity subtraction filter of a small-neighbourhood of 11x11 pixels ([Thomas et al., 2014]).

In all cases, a 3x3 Gaussian filter was also applied, since Gaussian lowpass filtering proved to be effective in improving the images signal-to-noise ratio ([Thomas et al., 2010, Scarano, 2013, Thomas et al., 2014]).

Figure 5.1 gives a sample of the original camera-1 image with a nonuniform illumination and light reflections of the laser light on the bottom glass of the wind tunnel and the remarkable improvement of particle contrast after the subMedian and subMean filters. The subMin background subtraction was not capable to remove light reflections. For other experimental data with high differences of illumination along the time-history a short timehistory subMedian subtraction as proposed by [Thomas et al., 2014] can be necessary. Other more advanced techniques for background removal for diffuse background reflections can be necessary, such as the modified local-median normalization algorithm was developed by [Mejia-Alvarez and Christensen, 2013].

Figure 5.2 presents the results for the pre-processing filters tested regarding the reconstruction light of the illuminated region. In the figure the normalized average intensity profile is presented for the six pre-processing filters described. The profiles were normalized by the maximum intensity inside the illuminated region, and are presented as a function of the thickness of the illuminated volume shown in the abscissa. A perfect reconstruction of a uniformly illuminated volume should display a distribution of the normalized intensity similar to the light illumination distribution, as already mentioned in section 3.3.4. Intensity levels outside the illuminated region are associated with ghost particles produced by the reconstruction algorithm. Six normalized intensity profiles are presented in Figure 5.2, associated to symbols that are cued to the pre-processing filters in the legend.

Two features of the results presented in Figure 5.2 are analysed. These are the shape of the intensity profile and the light intensity signal-to-noise ratio quality indicator described in section 3.3.4. As can be verified in the figure, all tomo-PIV reconstructions present a top-hat distribution as expected due to the use of knife-edge filter along the laser path in the experimental setup. The simple subMean pre-processing filter gives the best results. Indeed, the signal-to-noise ratio of 2.32 is the highest of all filters tested and above 2, the value recommended by [Scarano, 2013] for a good quality reconstruction. Also, the intensity levels obtained outside the illuminated volume are low, a desirable result that indicates the presence of a low number of ghost particles. 


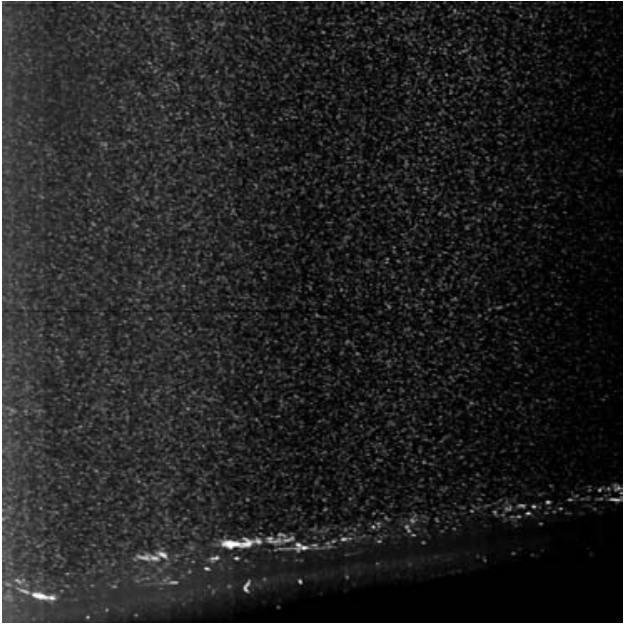

(a)

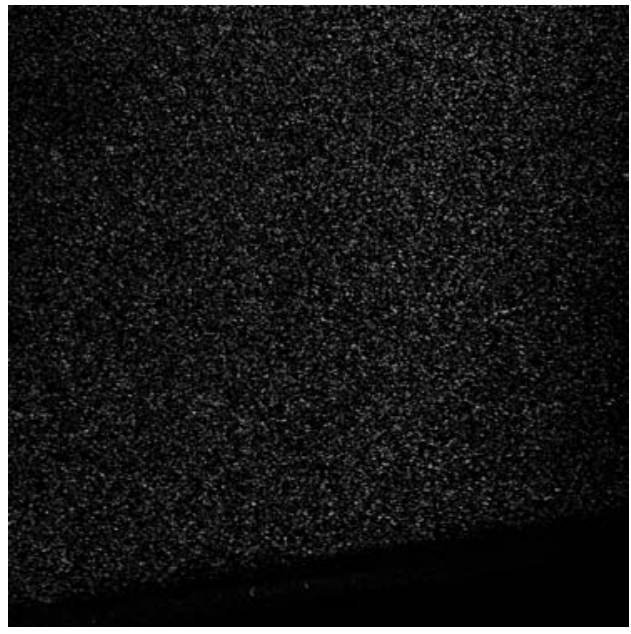

(c)

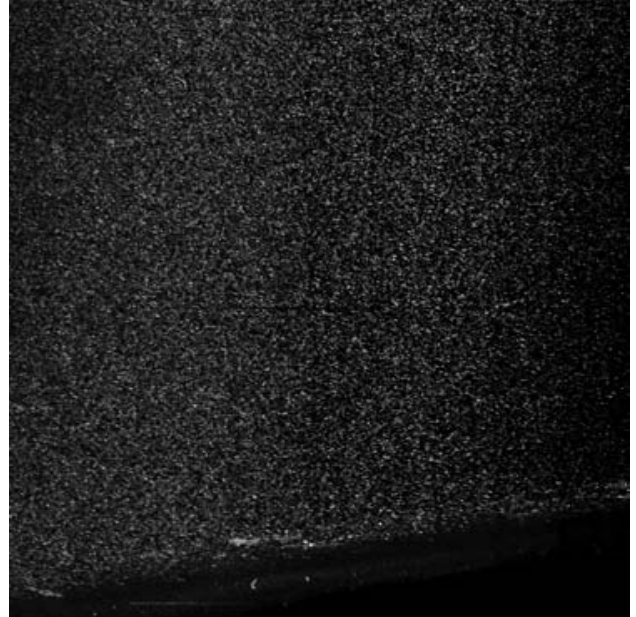

(b)

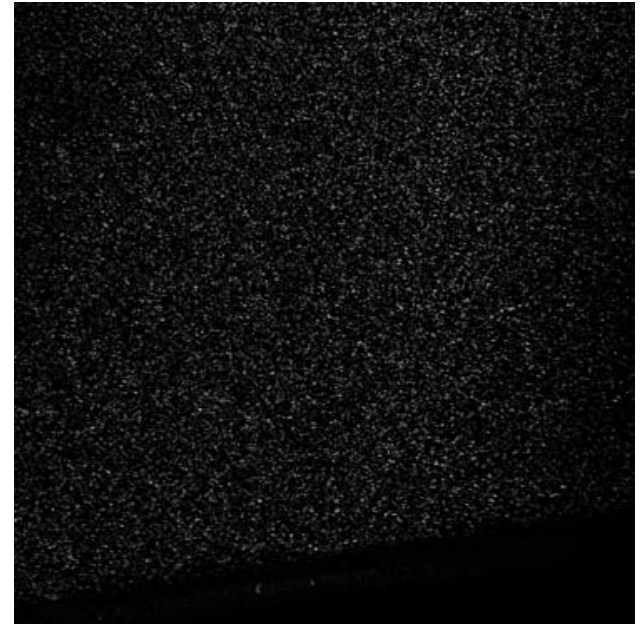

(d)

Figure 5.1: Sample of the original image from camera 1 (a) and after the background subtraction by subMin (b), subMedian (c) and subMean (d) filters. The images were normalized to enhance the visualization.

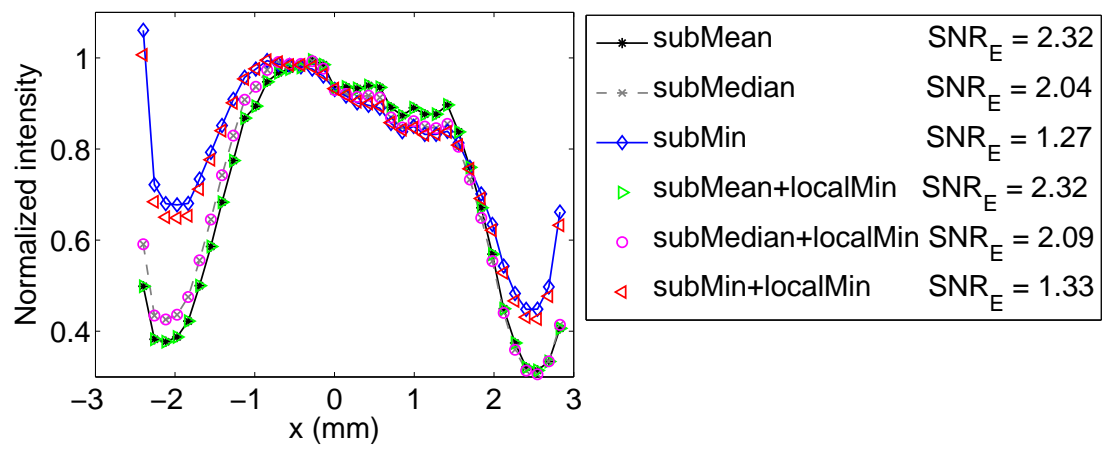

Figure 5.2: Influence of different background-subtraction filters combined with low-pass Gaussian filter on the intensity profile of reconstructed light for a 5-iteration standard MART. 
The use of minimum intensity processing in addition to the simple subMean, the +localMin, yielded virtually the same results as the subMean filter with an undesirable increment of the computational time. The poor performance of the subMin+localMin filter, suggested by [Thomas et al., 2010] and [Scarano, 2013], is attributed to the fact that the filter was unable to completely remove the light reflections due to variations of the reflection intensities in each image that composes the time-series analysed and, as a consequence, yielding a low $S N R_{E}$ below 2. The subMedian filter and its association with the minimum intensity subtraction filter displayed a performance between the subMean and subMin filters.

Regarding the normalized intensity variance, the lowest value of 16 was obtained for the image pre-processing using the subMin filter, while the highest value of 20, which is considered sufficient for a good reconstruction ([Lynch and Scarano, 2014]), was achieved employing the subMean filter. No significant changes were observed in the reconstructed particle characteristics. The reconstruction particle dimension of about 2.5 voxels was nearly the same along the three directions, satisfying the recommended range ([Raffel et al., 2007]). The obtained round shape of particles was expected from the 6-camera arrangement setup employing around $45^{\circ}$ tilt viewing angles.

\subsubsection{Volume Filtering}

In order to improve the quality of the reconstructed volumes, volume filters were utilized in the first two of the five reconstruction iterations of the standard MART algorithm employed in the tests. The filters tested were the volume threshold filter (vt) which brings down to zero voxel intensities under the threshold value, ([Atkinson and Soria, 2009]) and a volume Gaussian filter (vgf) of $1 \times 3 \times 3$ voxels, which smooths voxel intensity distribution, ([Discetti et al., 2013]). For the the present tomo-PIV setup, the test of a volume Gaussian filter of $3 \times 3 \times 3$ voxels would be recommended. Nevertheless this isotropic filter had not been implemented in the tomo-PIV software at the moment of this thesis.

Figure 5.3(a) presents the effect of these filters on the normalized intensity profile of the illuminated volume and on the light intensity signal-to-noise ratio quality criteria. The symbols in the figure indicate the different threshold levels tested for the threshold volume filter, varying from no threshold to 0.004, and if the volume Gaussian filter was activated or not. An analysis of the results presented in Figure 5.3(a) reveals that the use of the threshold filter is mandatory in this boundary-layer experiment. Indeed, volume filter with a threshold value of 0.004 appreciably improves the signal-to-noise ratio value, 
as compared to the case where no filter is employed, an improvement from 1.88 to 2.32 . The same trend was indicated by the normalized intensity variance ranging from 14 to 21 . Increasing the threshold value maintained only the brightest particles, as can be verified in Figure 5.3(b), where the probability density functions (PDF) of the normalized intensities of reconstructed particles inside the volume are presented. The average PDF of the $2 \mathrm{D}$ preprocessed images, using the subMean filter followed by a 3x3 Gaussian filter, is also plotted in the figure for reference purposes. The reconstructed volume without volume filters, labelled as $\mathrm{vt}=\mathrm{no}$ and $\mathrm{vgf}=\mathrm{no}$, shows particles highly concentrated at low intensity values. The volumes reconstructed using vt displays a more dispersed particle intensity distribution with a peak at higher intensity level, as compared to the reconstruction without volume filtering. The effect of thresholding the volume with a value of 0.004 is seen to spread the normalized intensity distribution a little more than the pre-processed image, displacing the intensity peak to higher levels. The changes observed in the PDF by using the volume threshold filter can impact on the performance of the cross-correlation procedure to calculate the velocity fields. As a consequence of the use of the volume thresholding filter, the total number of reconstructed particles decreased from 0.014 to $0.003 \mathrm{ppv}$, as can be verified in Figure 5.3(c), and so did the number of ghost particles. Also, the reconstructed particles remained relatively round, i.e., diameter aspect ratios $\left\langle d_{x}\right\rangle /\left\langle d_{z}\right\rangle$ and $\left\langle d_{y}\right\rangle /\left\langle d_{z}\right\rangle$ between 0.88 and 1 . The particle diameter remained at about 2.5 voxels. These results can be verified in Figure 5.3(d).

The use of an anisotropic 1x3x3 Gaussian filter notably increased the $S N R_{E}$ value up to 5.71. This can be observed in Figure 5.3(a). This increase is probably due to the attenuation and removal of ghost particles, as mentioned by [Discetti et al., 2013]. The PDF of the peak intensity shown if Figure 5.3(b) indicates that the use of the Gaussian filter produces a displacement of the maximum of the distribution toward higher intensity values, along with a spreading of the distribution. However, as can be seen in Figure 5.3(d), the particle aspect ratio $\left\langle d_{x}\right\rangle /\left\langle d_{z}\right\rangle$ of 0.57 was far from 1, while Figure 5.3(c) shows that the particle concentration is strongly reduced to only $0.001 \mathrm{ppv}$, what can be attributed to the filtering of some actual particles along with the ghosts. Anisotropic Gaussian filter might be useful to camera arrangements, other than the one employed in the present experiment, to correct for some elongated particle shapes. In the present case, these low-density, non-round reconstructed particles obtained with the use of the Gaussian filter would possibly harm the cross-correlation procedure employed to compute the vector field. For the present study where the particles are nearly round it is expected 

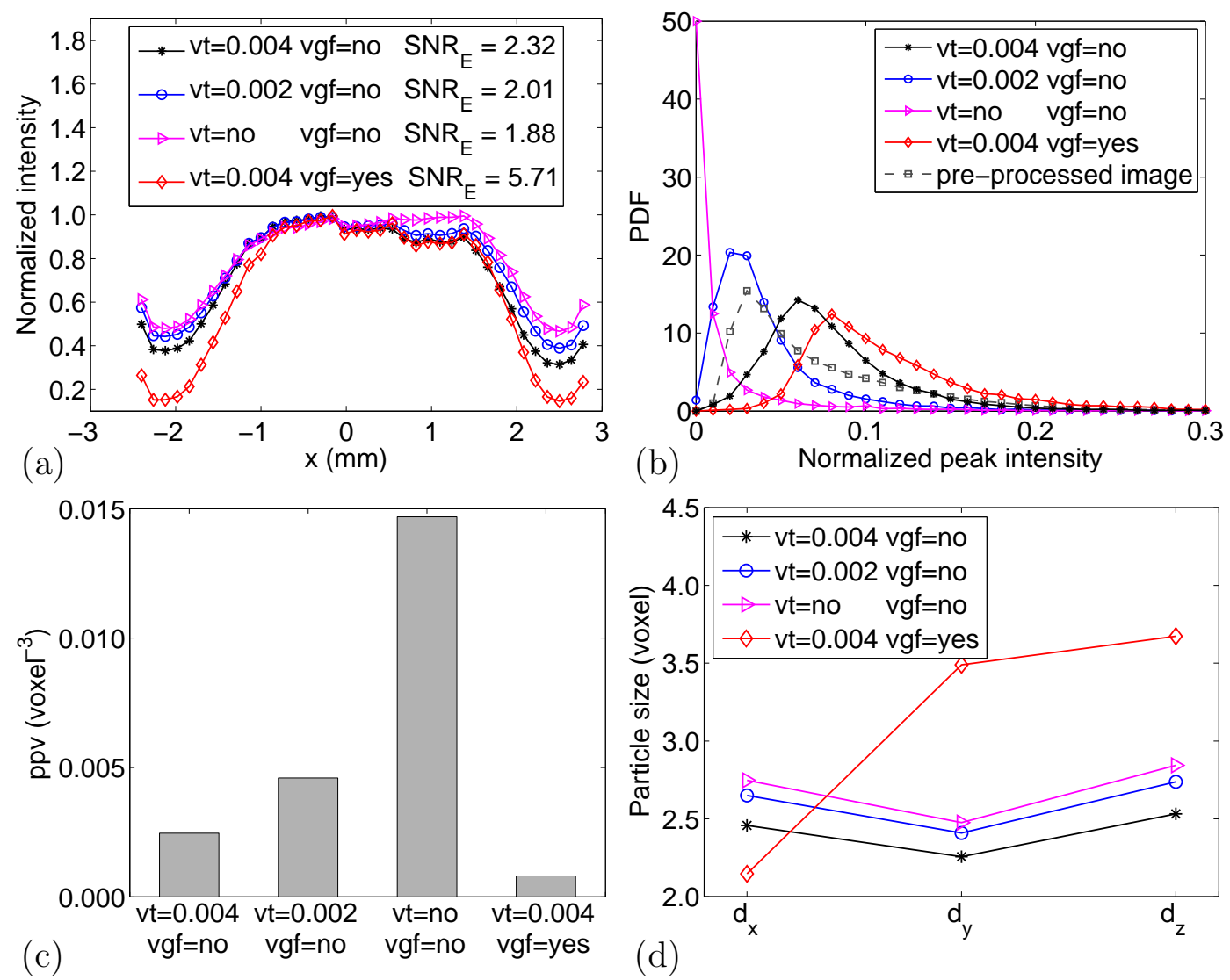

Figure 5.3: Influence of volume threshold filter (vt) and volume Gaussian filter (vgf) (a) on the intensity profile of reconstructed light, (b) on the probability density function of the normalized peak intensity, (c) on the particle concentration per volume and $(\mathrm{d})$ on the particle size for a 5 -iteration standard MART.

that a $3 \times 3 \times 3$ isotropic Gaussian filter, as that implemented by [Discetti et al., 2013] could improve the $S N R_{E}$ without deforming the particles. This filter was, however, not implemented. After the analysis of the optimization study using the quality indicators, in the present work, the volumes were filtered using a threshold of 0.004 and not using a Gaussian volume filter during the reconstruction. This choice looked as a good compromise between the number of reconstructed particles and the volume quality.

\subsubsection{Number of Cameras}

The number of cameras plays an important role in the volume reconstruction. Adding cameras means additional information on the particles, which increases the quality and allows for a higher seeding density ([Elsinga et al., 2006, Atkinson et al., 2011, Elsinga et al., 2011, de Silva et al., 2013, Scarano, 2013, Westerweel et al., 2013]). This fact can be verified by the normalized averaged intensity profiles of MART reconstructed volumes plotted in Figure 5.4(a). The quality increases progressively from three to six cameras 


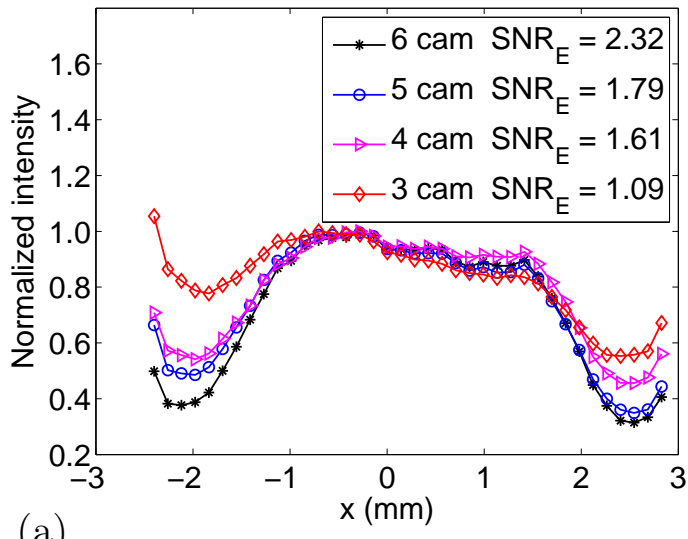

(a)

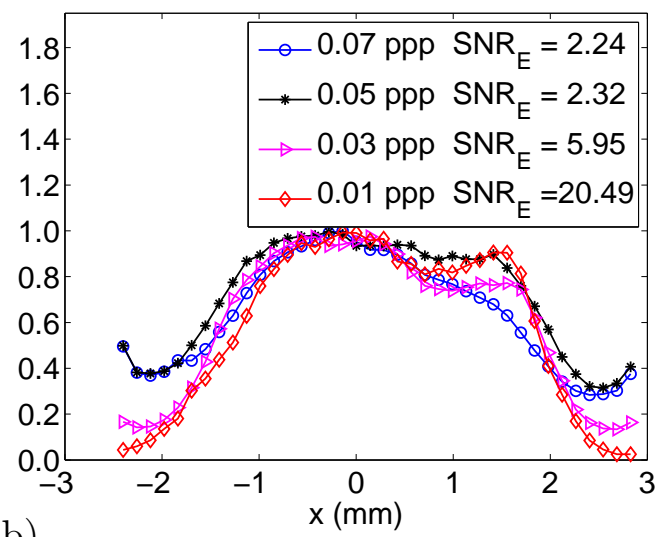

(b)

Figure 5.4: Influence of (a) the number of cameras and (b) the particle density on the intensity profile of the reconstructed light for a 5-iteration standard MART.

and the largest improvement is observed when four cameras are used instead of three (i.e., the $S N R_{E}$ level is almost $50 \%$ higher). Similar experimental results were obtained by other researchers ([Fukuchi, 2012, Foucaut et al., 2011a, Lynch and Scarano, 2014, Lynch and Scarano, 2015]).

It is important to mention that a higher signal-to-noise ratio was expected using 6 cameras viewing a moderate amount of particles inside a thin illuminated volume. The significant amount of ghost particles seems to be caused by illumination problems due to the low level of light intensity available for the experiment, taking into consideration that 2 kinds of cameras were used and the sensibility of Phantom cameras are probably not so high as the others. For this experiment, the use of 6 cameras seems mandatory to achieve the $S N R_{E}$ recommended in the literature ([Scarano, 2013]).

\subsubsection{Particle Density}

A larger number of particles is desired to improve the spatial resolution of the vector field ([Discetti et al., 2013]). Unfortunately the increase in particle density produces a larger amount of ghost particles, what decreases the reconstruction quality ([Elsinga et al., 2006]). Figure 5.4(b) shows the impact of the particle concentration on the normalized averaged intensity profile of the standard MART algorithm using six cameras. The concentration of the reconstructed particles in the volume, from 0.0002 to $0.0026 \mathrm{ppv}$, is related to the increase of the image particle density, from 0.01 to $0.07 \mathrm{ppp}$.

As the investigation volume is thin and the particle concentration is not high, the particle per pixel value was estimated by dividing the amount of local intensity peaks in particle distribution images by the respective image areas. Differently from the present experiment, for conditions in which particle 
superposition are present, a correction should be made in the mentioned local intensity peak calculation ([Fukuchi, 2012]) or a slit should be used in the experimental setup to shape the illumination region and indirectly calculate the $p p p$ by this thinner volume ([Lynch and Scarano, 2014]).

The difference on the signal-to-noise ratio between the volumes reconstructed with 0.01-ppp images and the ones with 0.07-ppp images was around 10 times. As expected according to simulations ([Thomas et al., 2014]), it is possible to exceed the concentration limit of 0.05 ppp, given by [Elsinga et al., 2006] for a 4-camera system, using more cameras in a standard MART reconstruction and still obtain an accurate result. Similar experimental conclusions were reached by [Lynch and Scarano, 2014]. Despite the fact that the sixcamera reconstruction of higher-density images gave a $S N R_{E}$ larger than 2, the conservative concentration of $0.05 \mathrm{ppp}$ was adopted in order to reach a lower uncertainty level in the velocity measurements.

\subsubsection{Summary of Tomo-PIV Optimization}

Optimization of tomo-PIV reconstruction of actual data sets was investigated using the following quality indicators: light intensity signal-to-noise ratio, normalized intensity variance, and reconstruction particle characteristics. The study was motivated by the fact that synthetic data have been employed as a means of guiding the optimization of actual experiments leading, however, to results that do not always present optimal performance. The quality indicators were applied to measurements obtained from a 6-high-speed camera boundary-layer experiment. Optimization studies were conducted in the image preprocessing and volume reconstruction phases, and proved to be valuable in improving the quality of the reconstructions. Optimization in these phases of the process when applied to small data samples can lead to better quality velocity results. Also, the use of quality indicators in the early stages of the measurement campaign allow that physical changes in the experimental setup be still implemented in case the optimized reconstruction fails to meet the quality criteria recommended values.

Image pre-processing algorithms are necessary to improve particle images located near the wall. Among the different filters tested, subtraction of timeaveraged images computed from the complete set of instantaneous images, followed by a 3x3 Gaussian filter, yielded the best results.

Volume filters are known to potentially improve the quality of reconstructed volumes. The volume filters tested were the volume threshold and volume Gaussian filter, both used in the first two reconstruction iterations. The tests have shown that a volume filter with a small threshold value can accelerate 
the convergence and remove ghost particles leading to higher values of the light intensity signal-to-noise ratio and normalized intensity variance quality indicators. The same trend was observed with the Gaussian filter. However, the combination of both filters was seen to decrease the amount of actual particles, reducing the measurement accuracy.

The influence of the number of cameras and particle density on volume reconstruction was discussed. As expected, the quality of reconstruction improved progressively with the number of cameras, with the largest improvement observed when four cameras were used instead of three. Regarding the particle density, the quality of reconstruction decreased with the amount of particles. However, particle concentration of 0.07 ppp employing 6 cameras yielded good quality reconstructions.

\subsection{Comparison of Optimized Reconstruction Algorithms}

The next sections will present a comparison of MART-standard, MARTbilinear, BIMART and SMART reconstruction methods. Firstly, a convergence analysis is conducted to guarantee that the comparisons are done for converged results. Following, the four tomo-PIV algorithms are compared regarding quality indicators of the reconstructed volumes produced from the boundarylayer data. Further, velocity statistics and uncertainties obtained with these algorithms are presented and compared to hot-wire anemometer data in order to validate the velocity measurements.

In this work, another implementation of MART reconstruction algorithm, denoted by the name "bilinear", in which the voxel weight is computed by a bilinear interpolation ([Thomas et al., 2014]), was tested. For BIMART and SMART, the voxel weight function was computed the same way as in MART-standard, mentioned in section 3.2.5. Except for SMART, the initialization was done by the minimum line-of-sight. For the initialization of SMART reconstruction method, 4 iterations of a faster and less accurate implementation of SMART was employed ([Thomas et al., 2014]).

\subsubsection{Convergence of Reconstruction Algorithms}

The distinct tomo-PIV algorithms analysed require different number of iterations to achieve a converged reconstructed volume. In this work, the convergence was considered to be reached if the residue of the mean quality of the re-projected volume and, both, the residues of the averaged intensity inside and outside the illumination volume, were below $0.1 \%$. Prior tests indicated 


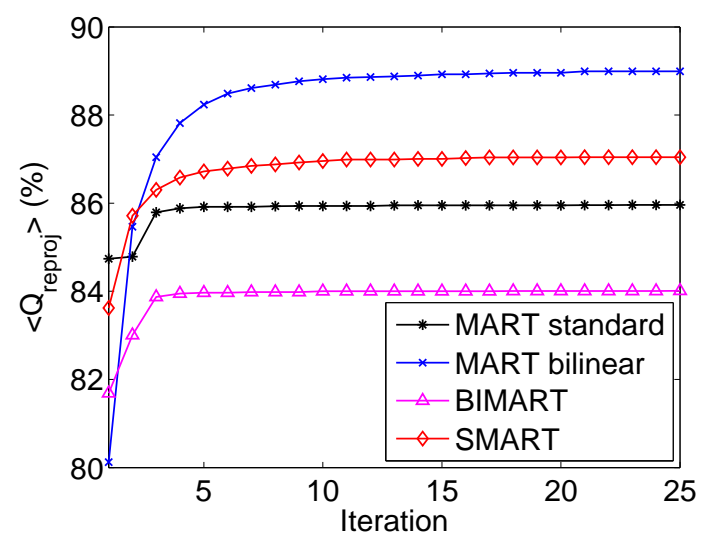

Figure 5.5: Convergence of mean re-projection quality for MART-standard, MART-bilinear, BIMART and SMART algorithms.

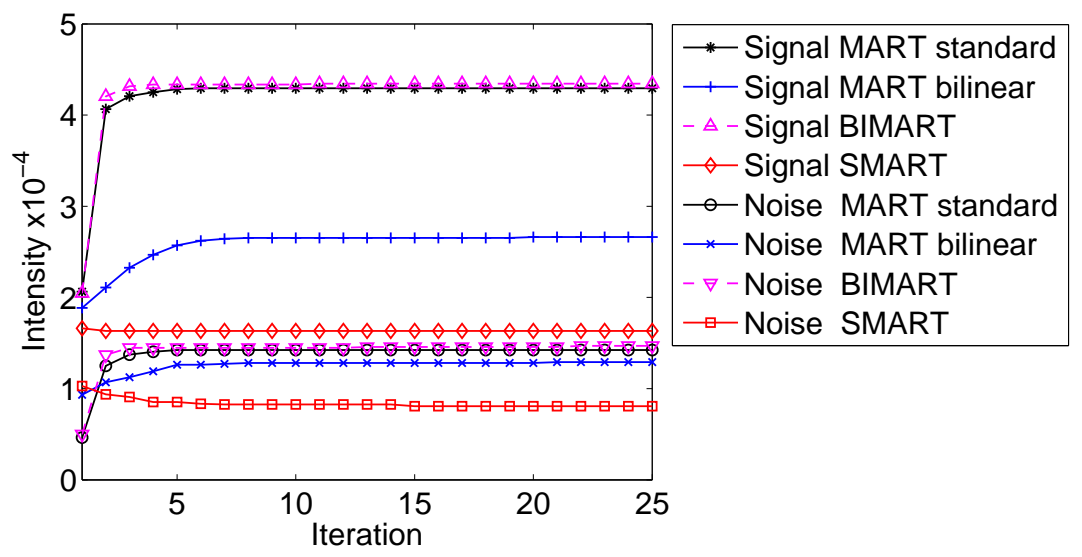

Figure 5.6: Convergence of $<E_{\text {in }}>$ (signal) and $<E_{\text {out }}>$ (noise) for MART-standard, MART-bilinear, BIMART and SMART algorithms.

that the $S N R_{E}$ converges faster than $<\boldsymbol{E}_{\text {in }}>$ or $\left\langle\boldsymbol{E}_{\text {out }}>\right.$ independently, so the use of the latter were considered more suitable for the analysis. Figures 5.5 and 5.6 show the convergence of the mean re-projection quality, the $\left\langle\boldsymbol{E}_{\text {in }}\right\rangle$ (related to signal) and $\left.<\boldsymbol{E}_{\text {out }}\right\rangle$ (corresponding to noise).

The convergence of the reconstructed light profile evaluated across the volume thickness can be observed in Figure 5.7 for all the reconstruction algorithms studied. In the figure, the intensity profile is presented for different number of iterations indicated in the legend. An expanded view of the different curves is displayed to facilitate the observation of convergence.

As usual, the MART-standard algorithm reached the established convergence criteria of $0.1 \%$ with 5 iterations. However, after 8 iterations no detectable variations were observed. This finding is in agreement with simulated PIV studies ([Thomas et al., 2014]). The converged averaged quality of the reconstructed volume, $\left\langle Q_{\text {reproj }}\right\rangle$, was $85.9 \%$, while the signal-to-noise ratio, $S N R_{E}$, was 2.32. Due to the use of a MinLOS "first guess" to initialize the reconstructed volume, the results suggest acceptable convergence, with a residue 
variations under $1 \%$, even with only 4 iterations, in agreement with [Worth and Nickels, 2008]. Nevertheless, for the present work, a conservative number of 5 iterations was adopted.

Comparatively, the MART with a bilinear iteration method applied to the experimental boundary-layer data used in the present work required more iterations for convergence than those required for the tests of [Thomas et al., 2014] using simulated data. For this specific experiment, stopping the algorithm with 5 iterations was considered premature. It converged only in 7 iterations and presented no measurable changes after around 26 iterations. The converged $<Q_{\text {reproj }}>$ was $89.0 \%$ and a poor $S N R_{E}$ of 1.87 was obtained.

The BIMART algorithm converged in 6 iterations and did not display significant changes after 8 iterations. The converged $<Q_{\text {reproj }}>$ was $84.0 \%$ and the $S N R_{E}$ was 2.42. This result corroborates the work of [Thomas et al., 2014].

For the SMART reconstruction method, the convergence was obtained with 15 iterations, in agreement with [Bilsky et al., 2011]. Around 28 iterations were required to obtain negligible changes in the reconstruction volume yielding a $\left\langle Q_{\text {reproj }}>\right.$ of $87.0 \%$ and a $S N R_{E}$ of 2.17. Again, for this case, similar behaviour was observed in the synthetic study of [Thomas et al., 2014].

\subsubsection{Reconstruction Algorithms}

Figure 5.8 compares the four tomo-PIV reconstruction algorithms tested. As mentioned before, the re-projection quality can not be used to quantify the reconstruction quality, nor is a simulated particle distribution available as a reference. So, the $S N R_{E}$ criterion was used to rank the reconstruction methods. As can be seen in Figure 5.8(a), the largest $S N R_{E}$ were obtained by BIMART and standard MART, followed by SMART. MART-bilinear did not yield good results. The normalized variance of the reconstructed intensity showed the same trend. The value of $\sigma_{E}^{*}$ was 21 for BIMART and the standard MART, 20 for SMART and only 11 for MART-bilinear.

As already commented, the MART-bilinear required 7 iterations to achieve convergence, implying in $22 \%$ more computational time as compared with the converged standard MART. Besides, it yielded the lowest $S N R_{E}$ and $\sigma_{E}^{*}$ values, under the acceptable limits for good reconstruction. This poor quality of the intensity profile could be explained by the large amount of low intensity particles (actual and ghost ones) inside the volume (i.e., $0.007 \mathrm{ppv}$ ) and the big diameter displayed by these particles of around 2.9 voxels (i.e., more than $15 \%$ of the standard MART particle diameters). These observations can be verified in figures 5.8(c) and 5.8(d). It should be mentioned that in the 
(a) MART-standard

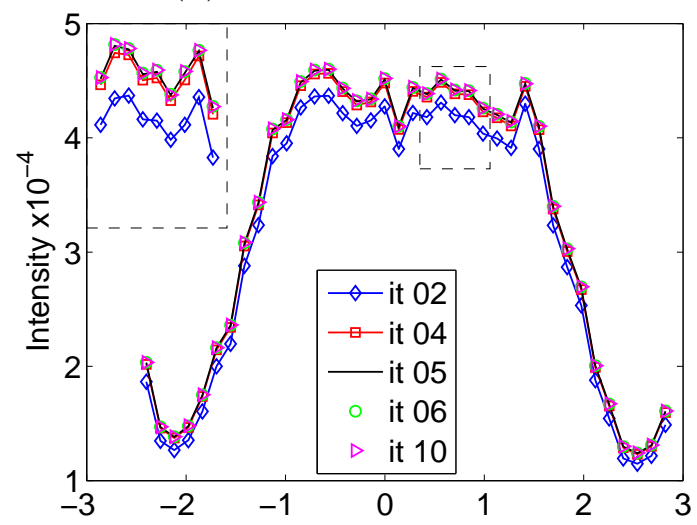

(c) BIMART

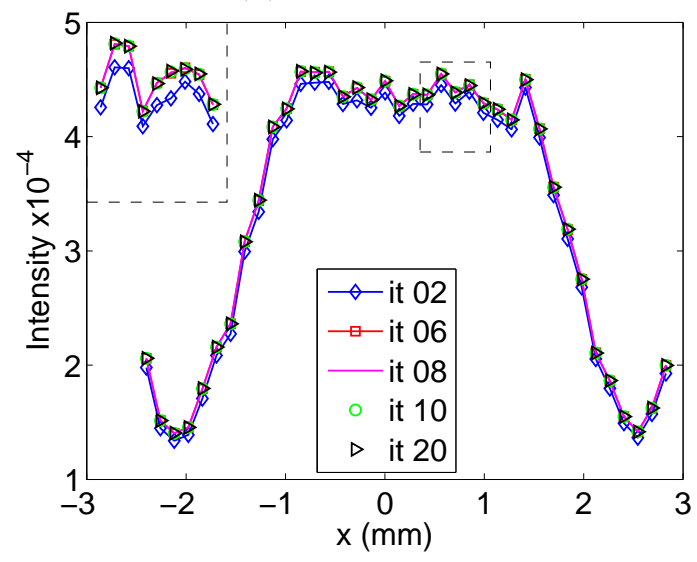

(b) MART-bilinear

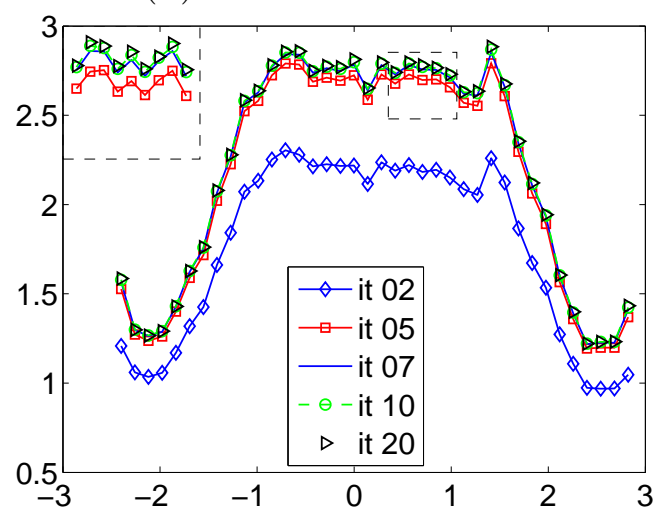

(d) SMART

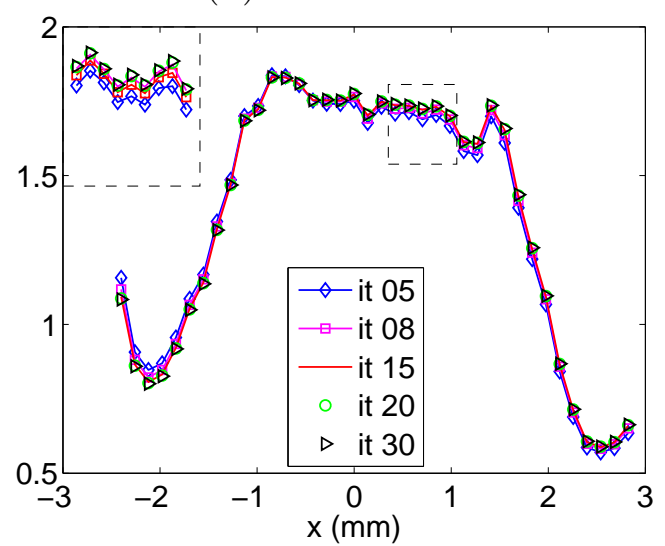

Figure 5.7: Convergence of reconstructed light profiles for (a) MART-standard, (b) MART-bilinear, (c) BIMART and (d) SMART algorithms.

synthetic tests of [Thomas et al., 2014], the MART-bilinear implementation presented a performance equivalent to that of the standard MART. The reasons for this different performance on synthetic and actual data should be better explored with additional experimental data sets. The studies conducted indicated that an increased value of the threshold of the volume filter could potentially improve the MART-bilinear performance regarding reconstruction particle concentration and diameter. These test were, however, not conducted in the present study.

Except for MART-bilinear, all the reconstruction algorithms displayed the same particle diameter of about 2.4 voxels in the $x$ and $z$ directions. In the $y$ direction, a compression of the particle diameters was observed, indicated by $\left\langle d_{y}\right\rangle /\left\langle d_{z}>\right.$ lower than 0.9 as can be seen in figure5.8(c). The results of Figure 5.8(d) show that SMART reconstructed $60 \%$ more particles than MART-standard and BIMART, both with $0.003 \mathrm{ppv}$.

The processing time required for the algorithms is presented in Figure 5.8(b). The time to perform MART reconstruction in a computer with a single AMD Opteron6128 $2 \mathrm{GHz}$ processor and $8 \mathrm{~GB}$ of RAM was about 
28 minutes, similar to the one reported by [Scarano and Poelma, 2009]. SMART was the fastest one (i.e., more than 3 times faster than standard MART), reconstructing the volume in only 9 minutes, approximately the same time found in the work of [Atkinson and Soria, 2009]. Nevertheless, [Thomas et al., 2014] indicated that the volume reconstructed by SMART seems to be slightly less accurate that the standard MART. The quality of reconstructed volume by BIMART with 8 iterations is equivalent to the volume quality of standard MART with 5 iterations. However, BIMART was 2 times faster and appears to be the best option for this experimental boundary-layer study. These conclusions are in consonance with the simulation test of [Thomas et al., 2014].

It is worth mentioning that the code implementation of the algorithms plays also an important role in the performance. The same algorithm, implemented by different groups, can produce distinct results due to some programming peculiarities, which are hard to quantify objectively. In the present work, the same code was used changing only the reconstruction method. So the same calibration, pre-processing image filters, volume filters and cross-correlation algorithm were employed in order to lead to a fair comparison.

\subsubsection{Velocity Statistics}

In this section, velocity results obtained from the processing of the boundary-layer data employing the different optimized reconstruction algorithms are presented. Although a 1-kHz, time-resolved, tomo-PIV experiment was carried out, the optimized reconstruction algorithms were applied to only 340 time-independent particle image pairs, producing vector fields spaced by $0.01 \mathrm{~s}$. This time interval was necessary to guarantee decorrelation of the captured flow fields, since the focus of the present analysis was on flow statistics.

The particle images from the six-camera arrangement were pre-processed by time-average background subtraction followed by a 3x3 pixels Gaussian filter. Prior tests showed that the use of a Gaussian filter of 3x3 pixels enlarge the particles and reduce the number of spurious vectors. The pre-processed images were reconstructed using 5 iterations of the standard MART with MinLOS initialization, a relaxation parameter of 0.2 and 2-iteration volumefilter threshold of 0.004 . The reconstructed volume was produced applying a pixel to voxel ratio of 0.8 and a volume enlargement along the thickness of $15 \%$ in order to increase the quality ([Thomas et al., 2014]). The final volumes were composed by $74 \times 644 \times 644$ cubic voxels with dimensions of $0.07 \mathrm{~mm}$. The MART reconstruction was compared with both the 8-iteration BIMART and the 15-iteration SMART. The BIMART used the same parameters as MART and a 

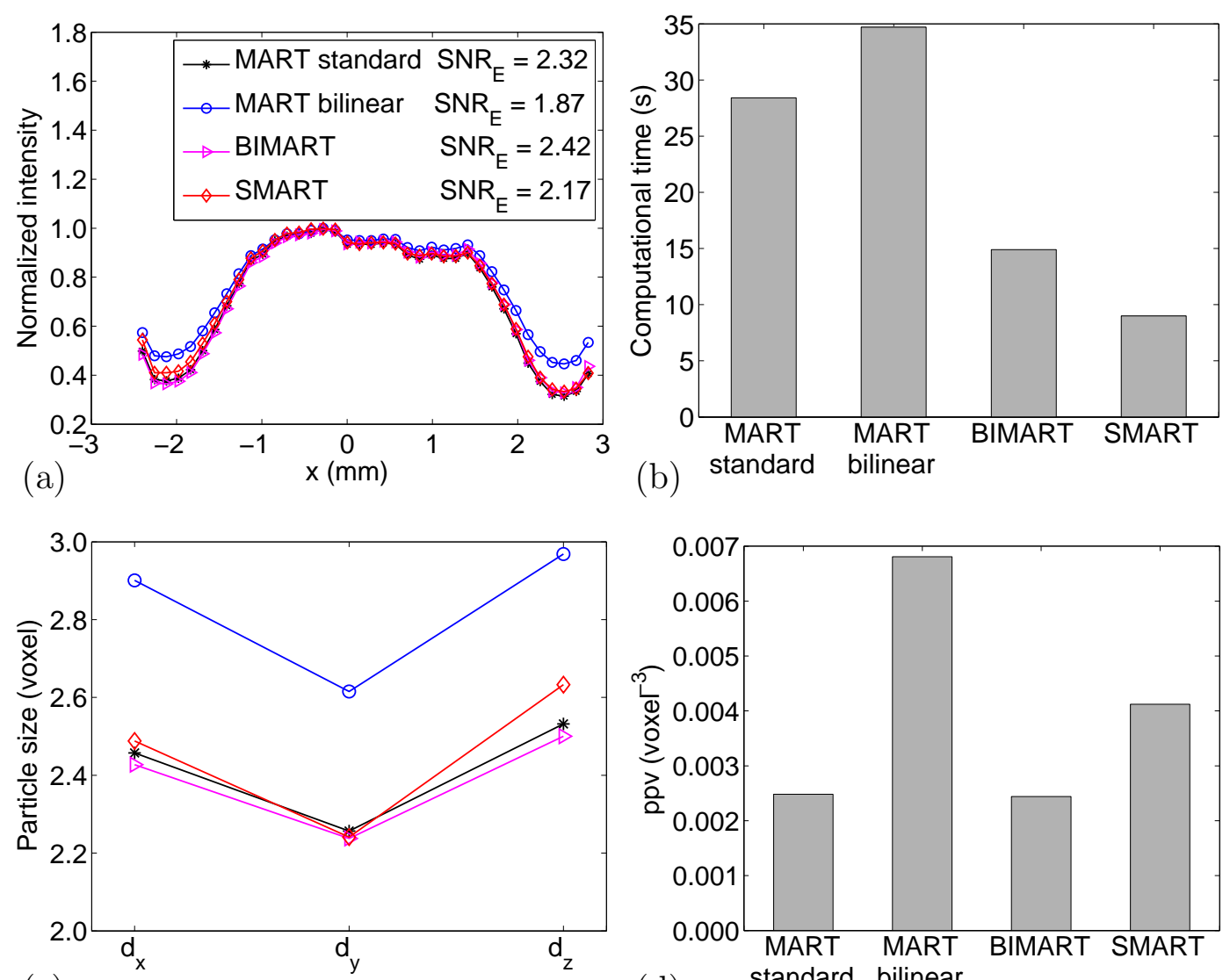

(c)

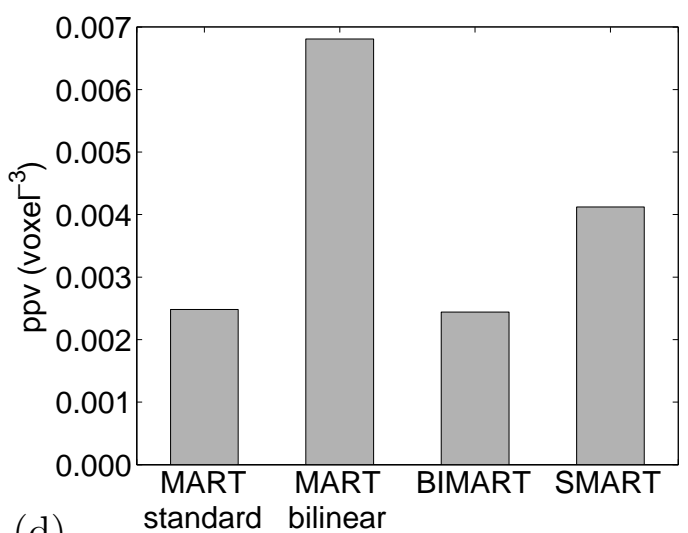

(d)

Figure 5.8: Comparison of (a) the averaged intensity profile, (b) the computational time required for one reconstruction, (c) the averaged particle size and (d) particle concentration for MART-standard, MART-bilinear, BIMART and SMART algorithms.

block size equal to four pixels, while SMART used as volume initialization the MinLOS method followed by 4 iterations of a faster SMART implementation and a relaxation parameter of 0.5 on the same pre-processed particle images.

The velocity fields were computed by means of a $3 \mathrm{D}$ multi-pass cross-correlation with sub-pixel shift from a pair of volumes containing the particle distribution, resulting in a final interrogation volume size of $36 \times 36 \times 36$ pixels with $75 \%$ of overlap. The final vector field grid was 5x68x68. The validation of the vectors was performed by means of median filtering, as proposed by [Westerweel, 1994]. The ratio of spurious vectors and the total number of computed vectors in all velocity volumes were equivalent. They were about $2.3 \%$ for MART and SMART, and $1.8 \%$ for BIMART.

The mean velocity uncertainty based on the divergence for MART and BIMART reconstruction methods was about 0.11 voxels, SMART being slightly above with 0.12 voxels. These good uncertainty values for a tomo-PIV air experiment were probably achieved due to the measurement in a narrow volume and the use of 6 cameras, being comparable to the values encountered 


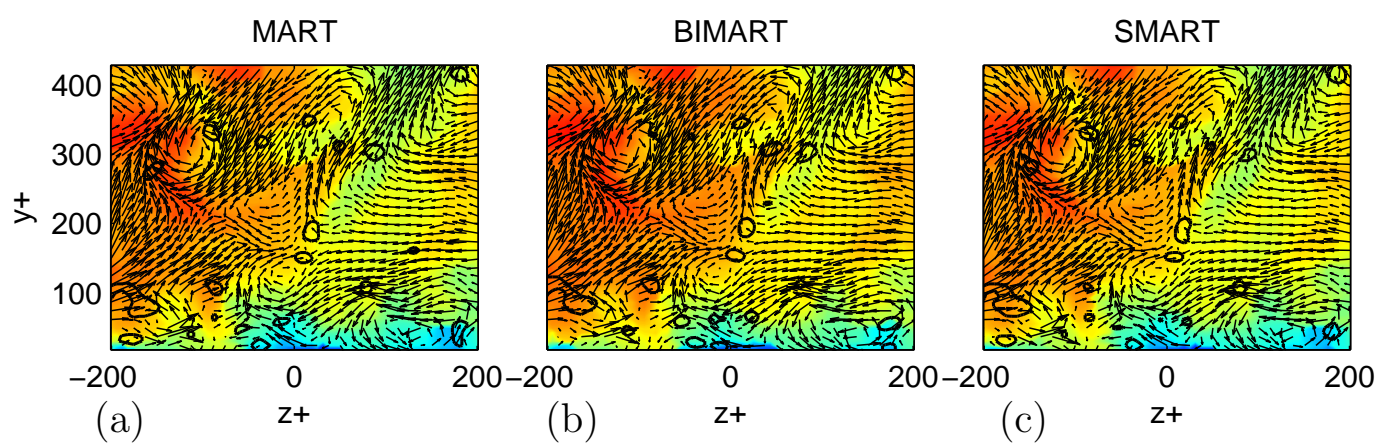

Figure 5.9: Comparison of instantaneous velocity field reconstructed by different methods: (a) MART, (b) BIMART and (c) SMART. The colour represents the third component and the black isolines the Q-criterion $=1500 \mathrm{~s}^{-2}$.

in the literature ([Scarano and Poelma, 2009, Atkinson et al., 2011]).

Unfiltered instantaneous velocity fields reconstructed by MART, BIMART and SMART from the same pair of particle images are presented in Figure 5.9. The Q-criterion ([Hunt et al., 1988]), which identifies vortices as flow regions with positive second invariant of the velocity gradient tensor, is represented as isolines in the same plots. An excellent agreement is observed in the velocity results of Figure 5.9 obtained with the three reconstruction methods. However, slight dissimilarities appear in the Q-criterion, although the locations of the major vortex are the same for the reconstruction methods.

The comparison of the velocity statistics from tomographic PIV reconstructions against the standard hot-wire anemometer data (HWA) from [Carlier and Stanislas, 2005] is presented in Figure 5.10. The log law in this plot is given by $U^{+}=(1 / \kappa) \log \left(y^{+}\right)+C$ with $\kappa=0.41$ and $C=5$. It can be verified that the tomo-PIV and HWA velocity profiles virtually collapsed, and that they perfectly follow the log-law curve.

Figure 5.10(b) shows a remarkable agreement between the profiles of turbulent velocity fluctuation components from tomo-PIV obtained with the three different reconstruction methods tested. Indeed, the small differences observed in the profiles from the three methods are within the expected uncertainty levels. The slight discrepancies observed between the tomo-PIV and the HWA data are attributed to the short time-history records acquired of $1.72 \mathrm{~s}$. Nevertheless, this record length was adequate for performing the comparison among the different tomo-PIV reconstruction methods. Also, it is observed that the differences are larger in regions close to the tunnel wall where light reflections are present. It should be mentioned that the measurements of $\sqrt{<w^{\prime 2}>} / u_{\tau_{w}}$ near the wall for the hot-wire are overestimated due to the size of the probe compared to the mean gradient at this region.

Figure 5.11 compares the probability density functions of the three 

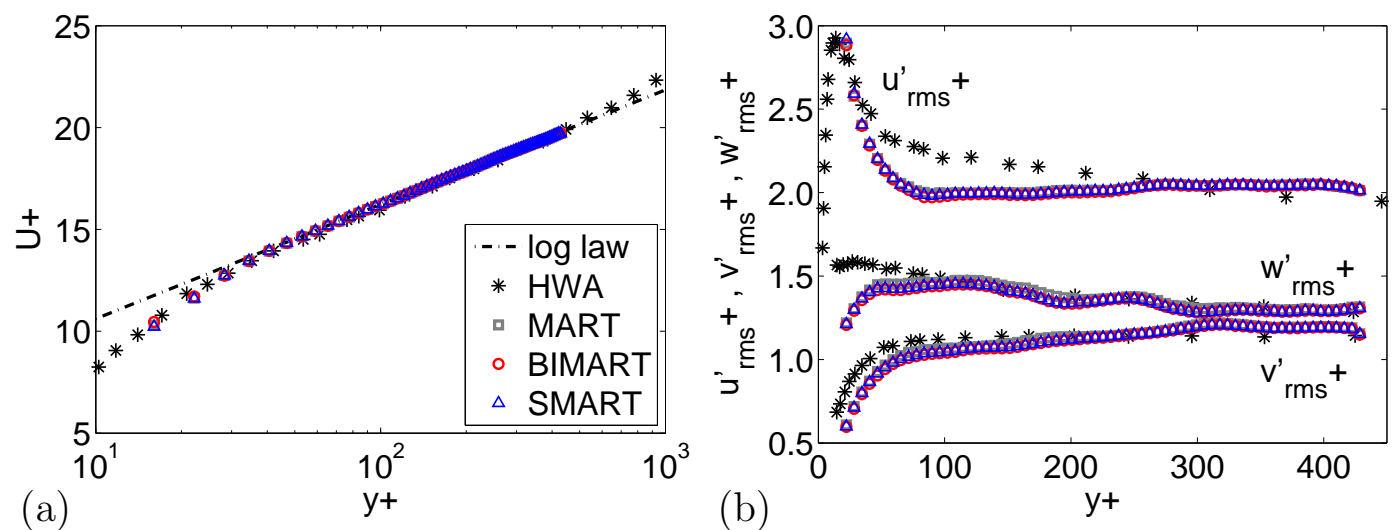

Figure 5.10: Plots of (a) velocity profile and (b) turbulence fluctuations normalized with respect of the viscous inner scale for MART-standard, BIMART and SMART algorithms.
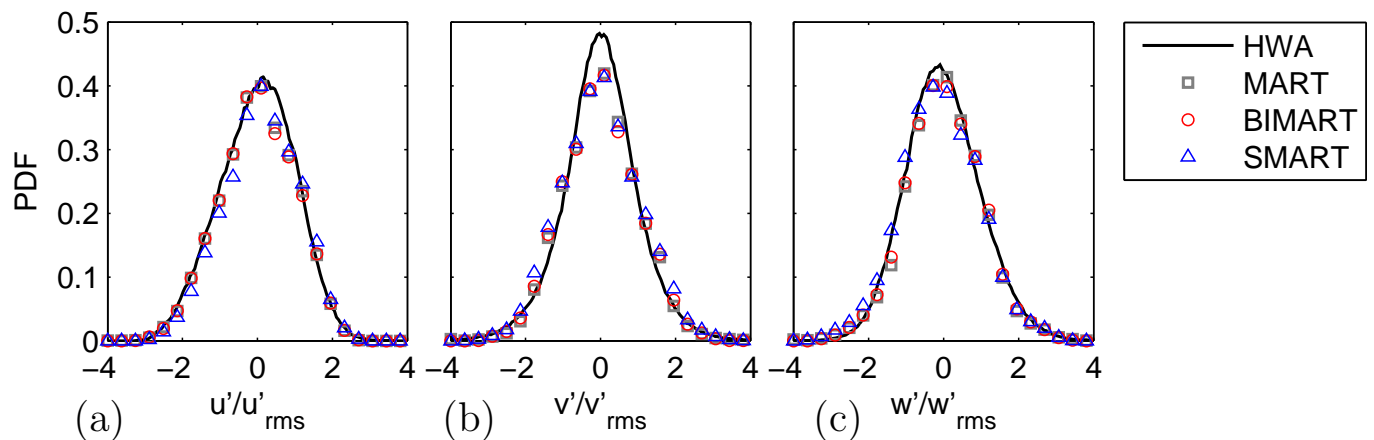

Figure 5.11: Probability density functions at $y^{+}=50$ for (a) $u^{\prime} / \sqrt{<u^{\prime 2}>}$, (b) $v^{\prime} / \sqrt{<v^{\prime 2}>}$ and (c) $w^{\prime} / \sqrt{<w^{\prime 2}>}$.

velocity components calculated at 50 wall units from the tunnel wall by tomo-PIV and HWA. The comparison shows excellent agreement among the reconstruction methods, and even with the HWA data. A marginal asymmetry in the $u^{\prime} / \sqrt{\left.<u^{\prime 2}\right\rangle}$ distribution is observed and can be attributed, seemingly, to the amount of data employed on the statistics. As a final comment, it should be mentioned that BIMART and SMART reconstruction algorithms are equivalent to the standard MART with the benefit of the reduction in computational cost.

\subsubsection{Comparison With Other Tomo-PIV Softwares}

The optimized tomo-PIV processing employing the software developed by Pprime, Coria and LML was compared to the commercial Davis software developed by LaVision GmbH. The Davis results were obtained by Dr. J. Vatteville, from LaVision, employing the guiding lines provided by the software for a wide range of experiments. Details of the two tomo-PIV processings are displayed in Table 5.1. 
Different camera calibration mapping functions were used. In the present work, cameras were calibrated by a pinhole model with a refraction correction from 7 equally-spaced calibration planes from $z^{*}=-3$ to $z^{*}=3 \mathrm{~mm}$ as described in chapter 4 . After the self-calibration procedure, the final projection errors were of the order of 0.01 pixel and maximum triangulation errors lower than 0.18 pixel. In the Davis software, also 7 equally-spaced calibration planes were employed, but with a third-order polynomial model. The initial projection errors of 0.12 to 0.17 pixel for all cameras and triangulation errors below 3 pixels were corrected by self-calibration method using only the brightest $10 \mathrm{k}$ particles in the images.

In order to improve the volume reconstruction quality and to reduce the time requirements, a meticulous analysis was performed in the present work (as described in the preceding sections). As a result, a 8-iteration BIMART with a block size equal to four pixels and with 3-iteration volume filter of threshold of 0.004 was applied to reconstruct volumes of $5 \times 45 \times 45 \mathrm{~mm}^{3}$. The 6-camera images were pre-processed using a time-average background subtraction followed by a $3 \times 3$ Gaussian filtering before the reconstruction procedure. From a pair of volumes comprising the particle distribution, the velocity fields were computed by means of a 3D multi-pass cross-correlation with sub-pixel shift. Two initial steps with an interrogation volume size of $36 \times 72 \times 72$ voxels were performed followed by three final steps with a interrogation volume size of 36x36x36 voxels and $75 \%$ of overlap. The resulting vector fields of $5 \times 67 \times 67$ grid were validated by a normalized median filtering and the spurious vectors, amounted to about $1.5 \%$, were successfully replaced.

LaVision used 5-iteration FAST MART, which is a variation of the standard MART, with 4-iteration volume Gaussian filter ([Discetti et al., 2013]) of $3 \times 3 \times 3$ voxels to reconstruct volumes of about $7 \times 83 \times 73 \mathrm{~mm}^{3}$. The 6 camera images were pre-processed using a background subtraction of a sliding minimum over $5 \times 5$ pixels, followed by a Gaussian smoothing and Sharpening filtering ([Gonzalez, 2009]) over 3x3 pixels before the reconstruction procedure. From a pair of volumes comprising the particle distribution, the velocity fields were computed by means of a 3D multi-pass direct cross-correlation with sub-pixel shift, volume deformation ([Scarano and Riethmuller, 2000]) and Gaussian weighting ([Nogueira et al., 1999]). The final interrogation volume size was $48 \times 48 \times 48$ voxels with $75 \%$ of overlap. The resulting vector fields of $10 \mathrm{x} 123 \mathrm{x} 138$ grid were validated by the universal outlier detection with threshold of 2 ([Westerweel and Scarano, 2005]) and the spurious vectors were replaced. Standard parameters, without optimization, were used in the LaVision software. 

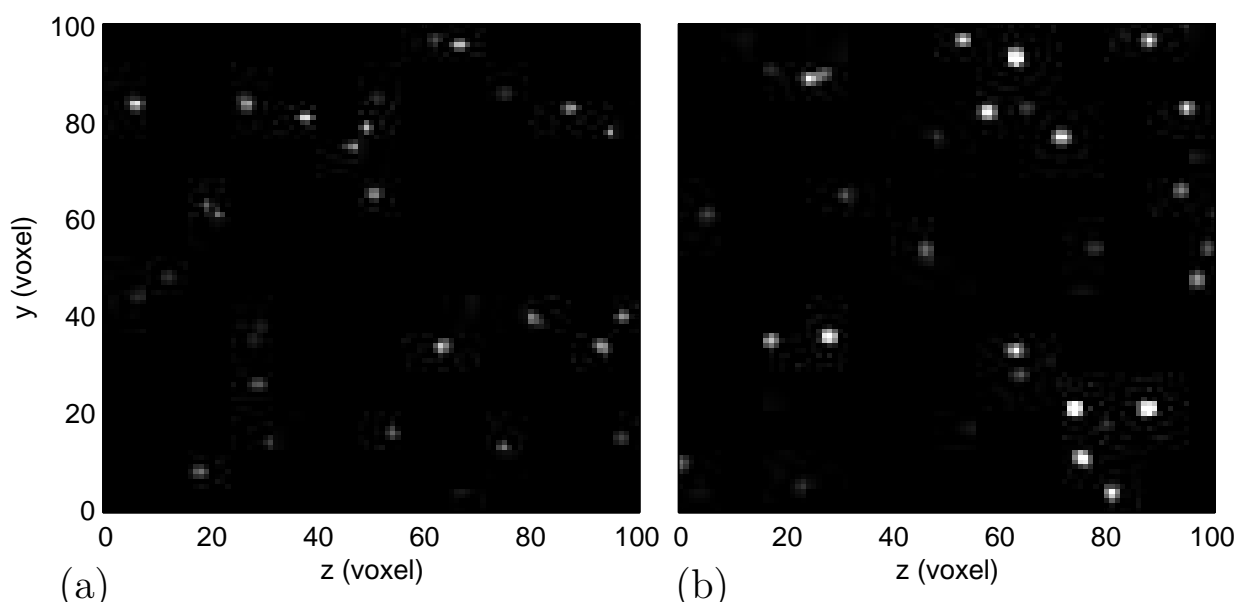

Figure 5.12: Comparison between reconstructed particles in the center region of the reconstructed volume computed by (a) the code of the present work and (b) Davis software.

For comparison purposes of reconstruction particles, figure 5.12 shows a sample of wall-normal-spanwise plane containing 100x100 voxels in the central part of an instantaneous reconstructed volume for the present work and Davis processing. The intensities were normalized for clarity. It is possible to observe that the present work reconstructed round particles of about 1 to 3 voxels while the processing in Davis software produced bigger particles of 2 to 4 voxels. This size difference seems to come from the volume filters used to remove ghosts. The threshold volume filter implemented in the present work decreases the particle diameter removing some of the tail of the intensity distribution of each particle. On the other hand, the Gaussian volume filter of $3 \times 3 \times 3$ voxels in the LaVision code increases the particle diameter smoothing its intensity distribution. Also the discretizations of imaged particles are distinct, because voxel sizes are different from both reconstructions (as can be seen in Table 5.1). It is worth to mention that the samples displayed in figure 5.12 are not at the same region, so comparisons of particle positions are not possible.

Figure 5.13 shows the middle plane of three instantaneous 3D-velocity fields at three consecutive times computed by the code of the present work and Davis software. The interrogation volume sizes used by both tomo-PIV processings comprises the minimum number of 5 to 10 particles needed within the interrogation region to ensure robust cross-correlation analysis ([Scarano, 2013]). Since the processing by Davis performed the motion analysis for a larger domain than that of the present work, its velocity field was cropped to nearly the same region as that of the analysis of the present work resulting in $8 \times 75 \times 75$ vectors. The larger amount of vectors compared to those obtained 
Table 5.1: Camera calibration, image pre-processing, tomographic reconstruction algorithm and cross-correlation details for the present work and Davis (LaVision).

\begin{tabular}{lcc}
\hline & Present work & Davis \\
\hline calibration & pinhole & polynomial fit \\
\hline image & time-average subtraction, & $5 \times 5$ sliding-minimum \\
pre-processing & $3 \times 3$ Gaussian filtering & $\begin{array}{c}\text { subtraction, 3x3 Gaussian } \\
\text { and Sharpening filtering }\end{array}$ \\
\hline
\end{tabular}

\begin{tabular}{lcc}
\hline $\begin{array}{l}\text { reconstruction } \\
\text { algorithm }\end{array}$ & $\begin{array}{c}\text { BIMART } \\
(\text { block size }=4)\end{array}$ & FAST MART \\
\hline
\end{tabular}

\begin{tabular}{lcc}
\hline iterations & 8 & 5 \\
\hline reconstruction & $74 \times 644 \times 644$ & $117 \times 1661 \times 1471$ \\
volume $\left(\right.$ voxel $\left.^{3}\right)$ & & \\
\hline voxel size $(\mu \mathrm{m})$ & $70 \times 70 \times 70$ & $50 \times 50 \times 50$
\end{tabular}

volume threshold filtering of $0.004 \quad 3 \times 3 \times 3$ Gaussian filter

post-processing (first 3 iterations) (first 4 iterations)

cross-correlation iterative multi-grid direct correlation by

algorithm with subpixel displacement iterative multi-grid with volume deformation and

Gaussian weighting

cross-correlation $36 \times 36 \times 36$ with $75 \%$ overlap $48 \times 48 \times 48$ with $75 \%$ overlap volume $\left(\right.$ voxel $\left.^{3}\right)$

\begin{tabular}{lcc}
\hline vector grid $(\mathrm{mm})$ & 0.64 & 0.60 \\
\hline $\begin{array}{l}\text { total amount } \\
\text { of vectors }\end{array}$ & $5 \times 67 \times 67$ & $10 \times 138 \times 123$ \\
\hline vector & $5 \times 5 \times 5$ median filtering, & $3 \times 3 \times 3$ universal \\
post-processing & $3 \times 3 \times 3$ bilateral filtering & outlier detection, \\
& $($ after each pass) & $3 \times 3 \times 3$ smoothing \\
& & $($ after each pass) \\
\hline
\end{tabular}


in the present work was possible due to the voxel size and the reconstruction of a larger domain. To enhance the visualization, only one over two vectors are displayed. As can be seen in the figure, the vectors shows the same flow behaviour and only slight differences are locally observed. Well-behaved vectors near the wall indicate that the image pre-processing was efficient for the mitigation of light reflections on the surface. Similar results, not presented in this manuscript, were also obtained for vorticity and Q-criterion detection ([Hunt et al., 1988]) of turbulent structures.

The tomo-PIV code used in the present work was also confronted against other softwares on experimental and synthetic tests in the framework of the $4^{\text {th }}$ International PIV Challenge ([Kähler et al., 2016]). It achieved good results in the main categories analysed showing its capability of accurately measuring different types of flow. 

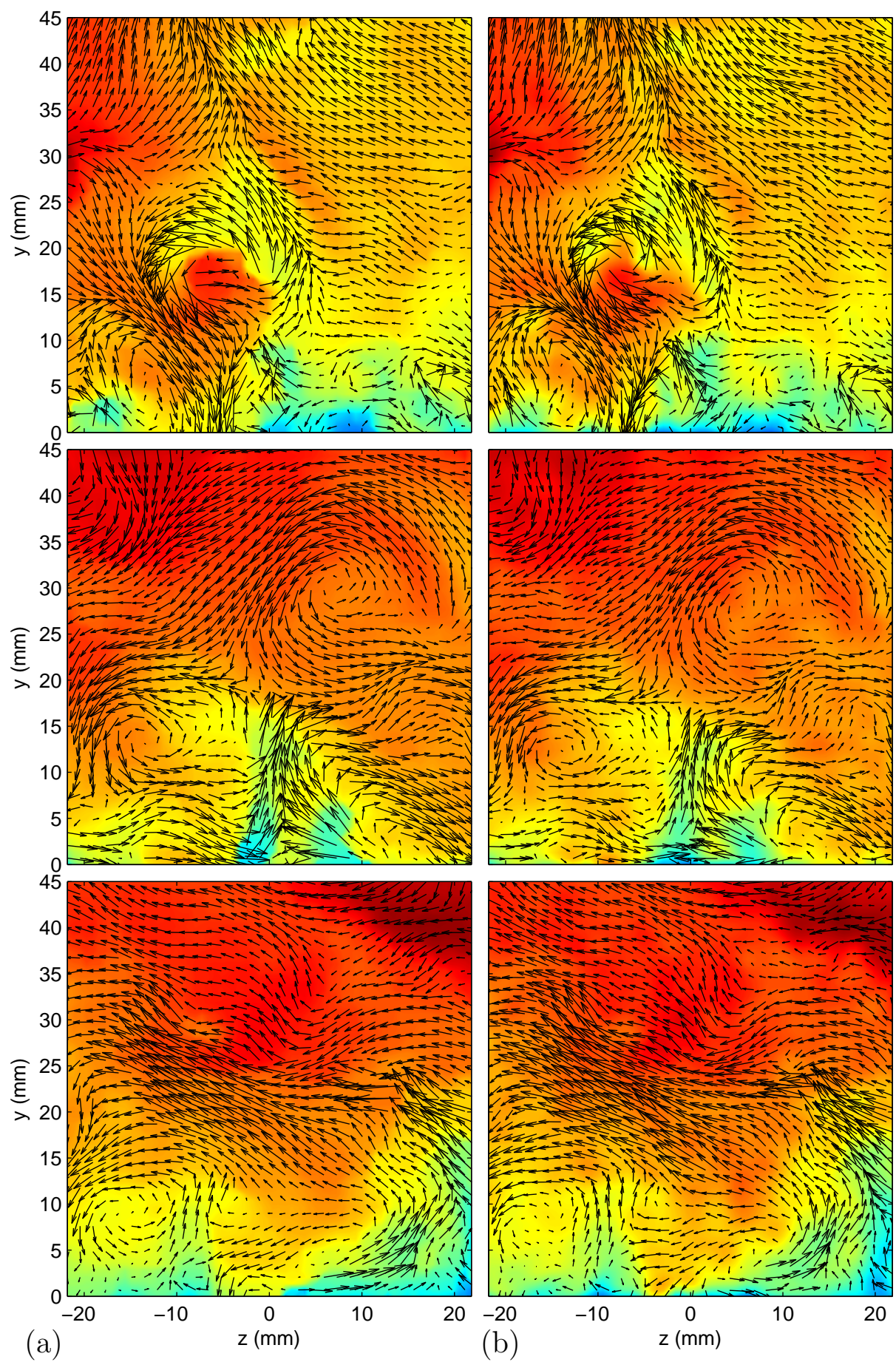

Figure 5.13: Comparison between instantaneous velocity fields computed by (a) the software of the present work and (b) Davis software. The colour represents the third velocity component. From top to bottom, each row of figures represents, respectively, time instants $0.005 \mathrm{~s}, 0.605 \mathrm{~s}$ and $1.205 \mathrm{~s}$. 


\subsubsection{Summary of the Comparison of Algorithms}

A comparative study of four optimized reconstruction algorithms was performed using the boundary-layer data. These are the MART-standard, MART-bilinear, BIMART, and SMART reconstruction methods. Convergence tests were conducted in order to guarantee that the comparisons were performed for converged results. BIMART and MART-standard yielded the largest light intensity signal-to-noise ratio values, followed by SMART. MARTbilinear did not yield good results. Except for MART-bilinear, all algorithms produced about the same particle diameters with low degree of distortion. SMART required about one third, and BIMART half, of the processing time required by MART.

Velocity statistics obtained with the different optimized tomo-PIV algorithms were compared with hot-wire data. MART, BIMART and SMART algorithms presented the same level of agreement with the hot-wire data. Likewise, uncertainty levels in the velocity measurements were about the same for the three methods. Well-tuned BIMART and SMART algorithms produced equivalent results to the state-of-the-art MART, with a significant reduction in computational time.

The comparison of the present tomo-PIV code with other softwares presented good results, showing the capability of this tomo-PIV processing in accurately measuring the flow properties. The 4D-3C velocity fields enable the computation of the full velocity gradient tensor and the reconstruction of the time history of turbulent structures. 


\section{6 \\ Boundary Layer Results}

This chapter discuss statistical results obtained from the tomo-PIV processing of the boundary-layer experiment in the LML wind tunnel. Timeresolved tomo-PIV velocity fields were post-processed employing a Matlab code especially developed for statistical analysis. Instantaneous visualizations are presented prior to this statistical analysis. Flow statistics were studied and compared to conditional statistics of low-speed, high-speed, ejection, sweep and vortex regions. The pattern recognition procedure developed to classify these turbulent structures and the extraction features procedure employed to compute conditional statistics are also presented.

The present chapter produced the following publications:

Martins, F. J., Foucaut, J. M., Azevedo, L. F., and Stanislas, M. (2015). Near-Wall Study of a Turbulent Boundary Layer Using High-Speed Tomo-PIV. In Progress in Wall Turbulence 2 (pp. 347-356). Springer International Publishing.

Martins, F. J., Foucaut, J. M., Azevedo, L. F., and Stanislas, M. (2015). Near-wall study of a turbulent boundary layer using time-resolved tomo-PIV. In $11^{\text {th }}$ International Symposium on Particle Image Velocimetry - PIV15, Santa Barbara.

\subsection{Overall Flow Statistics}

Tomo-PIV technique was used in the LML wind tunnel to study the nearwall region of a turbulent boundary-layer as described in chapter 4. Tomo-PIV acquired images were processed to produce 3D velocity fields after a thorough optimization study, detailed in chapter 5. As already mentioned, the particle image data were obtained before the author went to LML ([Foucaut et al., 2011a]).

The particle images from the six cameras were pre-processed by timeaverage background subtraction followed by a 3x3 Gaussian smoothing. The pre-processed images were reconstructed using 8 iterations of the BIMART with MinLOS initialization, a block size equal to four pixels, a relaxation parameter of 0.2 and 2 iterations of the volume filter with a threshold of 
Table 6.1: Tomo-PIV reconstructed volume characteristics.

\begin{tabular}{ccc}
\hline Field of view & Reconstructed volume & Interrogation volume \\
\hline $5 \times 45 \times 45 \mathrm{~mm}^{3}$ & $74 \times 644 \times 644$ voxel $^{3}$ & $36 \times 36 \times 36$ voxel \\
$50 \times 450 \times 450$ wall units & $50 \times 450 \times 450$ wall units $^{26 \times 26 \times 26 \text { wall units }}$ \\
\hline
\end{tabular}

Table 6.2: Tomo-PIV velocity field characteristics.

\begin{tabular}{cccr}
\hline Field of view & Grid space & Vectors per map & Maps \\
\hline $5 \times 45 \times 45 \mathrm{~mm}^{3}$ & $0.65 \times 0.65 \times 0.65 \mathrm{~mm}^{3}$ & $5 \times 68 \times 68$ & 8620 \\
$50 \times 450 \times 450$ wall units & $6.5 \times 6.5 \times 6.5$ wall units & & \\
\hline
\end{tabular}

0.004. The 3D reconstructed volume was produced employing a pixel to voxel ratio of 0.8 and a volume enlargement along the thickness of $15 \%$ in order to increase the quality. The final volumes were composed by $74 \times 644 \times 644$ cubic voxels with side dimensions of $0.07 \mathrm{~mm}$.

Instantaneous velocity fields were computed by means of a 3D multipass cross-correlation with sub-pixel shift from a pair of volumes containing the particle distribution, resulting in a final interrogation volume size of $36 \times 36 \times 36$ voxels with $75 \%$ of overlap. The amount of reconstructed particles inside the final interrogation volumes was between 5 to 10 checked by visual inspection. The time delay employed of $300 \mu$ s led to an average particle displacement of about 10 voxels. The final vector field grid was $5 \times 68 \times 68$. The validation of the vectors was performed by means of median filtering ([Thomas et al., 2014]). The ratio of the number of spurious vectors to the total number of computed vectors was about $1.5 \%$. Tables 6.2 and 6.1 summarize the data characteristics, regarding field of view (along $x, y$ and $z$ directions), reconstructed volume, interrogation volume size, grid space (distance between adjacent vectors), amount of vectors inside velocity maps and number of velocity fields (in 5 experimental runs).

A 4D-3C data post-processing software, written in Matlab language, was developed for the present study. This home-made software was capable of extracting statistics of the flow and of turbulent structures and also to display the data as $2 \mathrm{D}$ and $3 \mathrm{D}$ plots.

Figure 6.1 presents samples of tomo-PIV velocity field of the boundarylayer experiment. Three orthogonal slices of the velocity fields coloured by the streamwise velocity component are shown in this figure. On the middle plane, besides the streamwise velocity colour, 2D velocity vectors, expressing the wall-normal and spanwise components, are plotted. Just one over three vectors were drawn for clarity. "Parula" ([Alec's Web Log, 2014]) colormap 


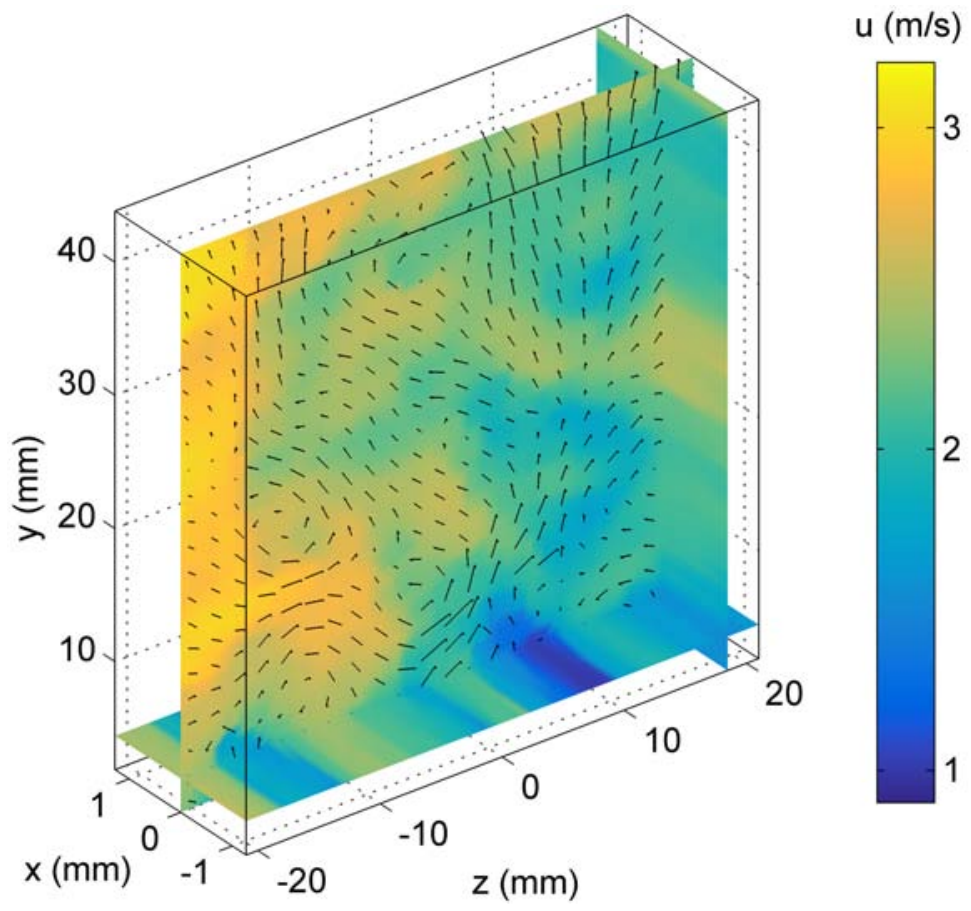

Figure 6.1: Sample of 3D tomo-PIV velocity field in the boundary-layer experiment. The color code refers to the streamwise velocity. One over three $2 \mathrm{D}$ vectors of wall-normal and spanwise velocity components are also plotted.

instead of the standard Matlab "Rainbow" was adopted after some suggestions on the literature about scientific visualization ([Bergman et al., 1995, Rogowitz and Treinish, 1998, Borland and Taylor II, 2007, Moreland, 2009]).

Flow details can be observed in 2D plots. Samples of instantaneous velocity fields on the middle plane $(x=0)$ of the volume (same slice of the volume displayed in figure 6.1) are shown in figure 6.2. From this figure, it is possible to observe the good spatial resolution of the present time-resolved tomo-PIV measurements. Different sizes of vortices can be inferred from the rotatory movement of wall-normal and spanwise vectors. The bottom region of the flow seems to be more influenced by the wall (at $y=0)$. At this region, crests marking the low-speed streaks (dark blue) and vortices are observed.

Since the boundary-layer tomo-PIV data from the present experiment were time resolved, 3D plots, with the third dimension being time, complement the 2D analysis, helping as a tool in the inference of turbulent structure dynamics and evolution. In favour of the vortical-structure clarification, the elimination of noise in the vortex detection was made by a robust discretecosine-transform filter [Garcia, 2010] followed by a volume filtering in the labelled field. The visualization of low-speed streaks together with vortical structures (employing the Q-criterion detection defined in eq. (6.2.24) according to [Hunt et al., 1988]) is displayed in figure 6.3. From this sample, it is possible to identify vortices neighbouring the low-speed streaks. The close 


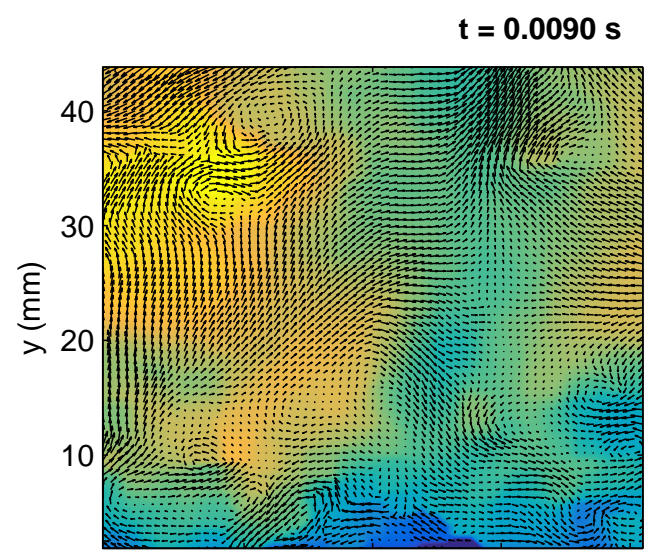

$t=0.0110 s$
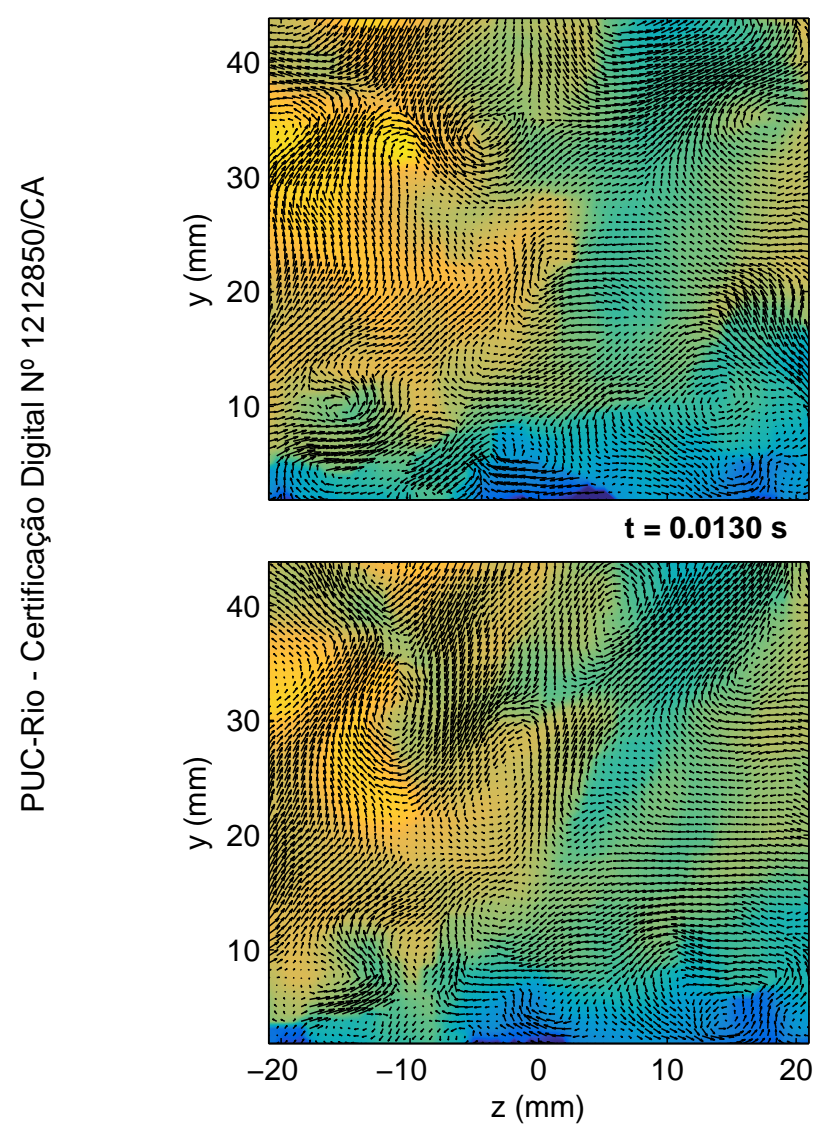
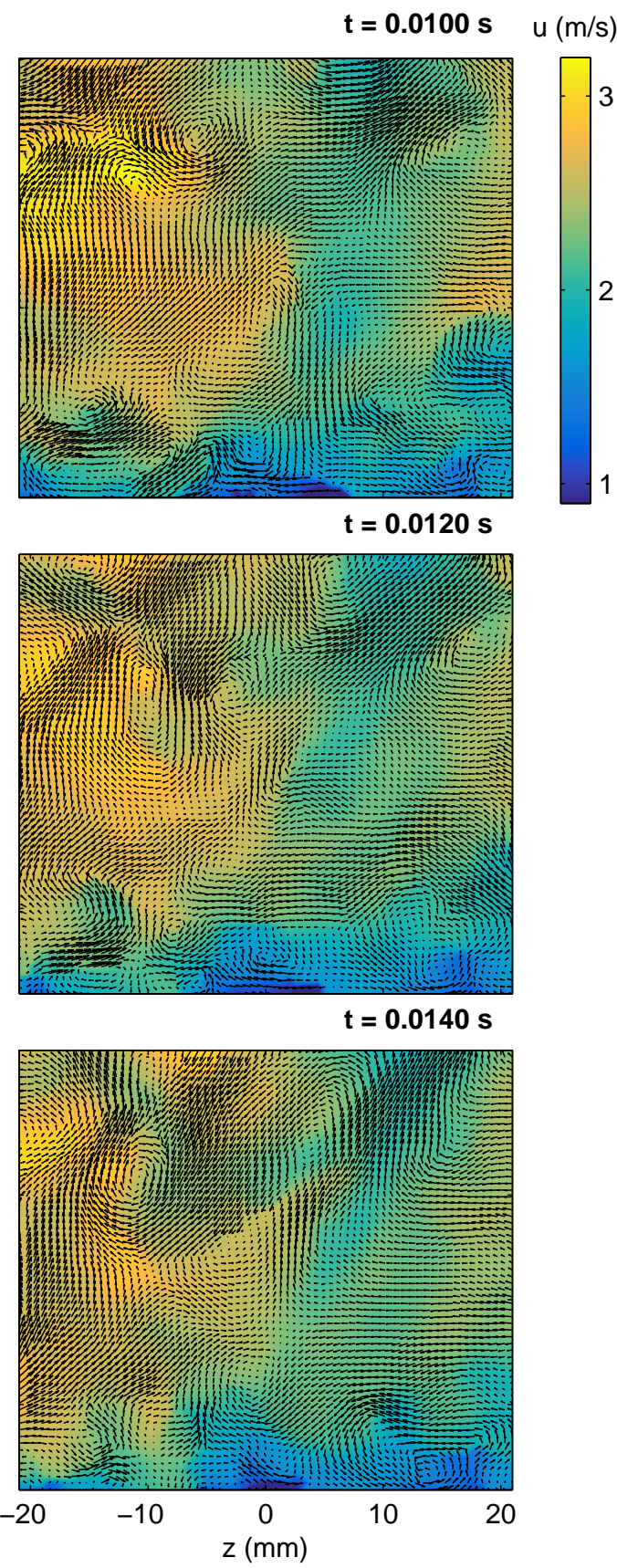

Figure 6.2: Six consecutive instantaneous velocity fields on the middle plane of tomo-PIV measurements of the boundary-layer experiment. Colour code related to the instantaneous stream-wise velocity and vectors related to the wall-normal and spanwise instantaneous velocity components. 

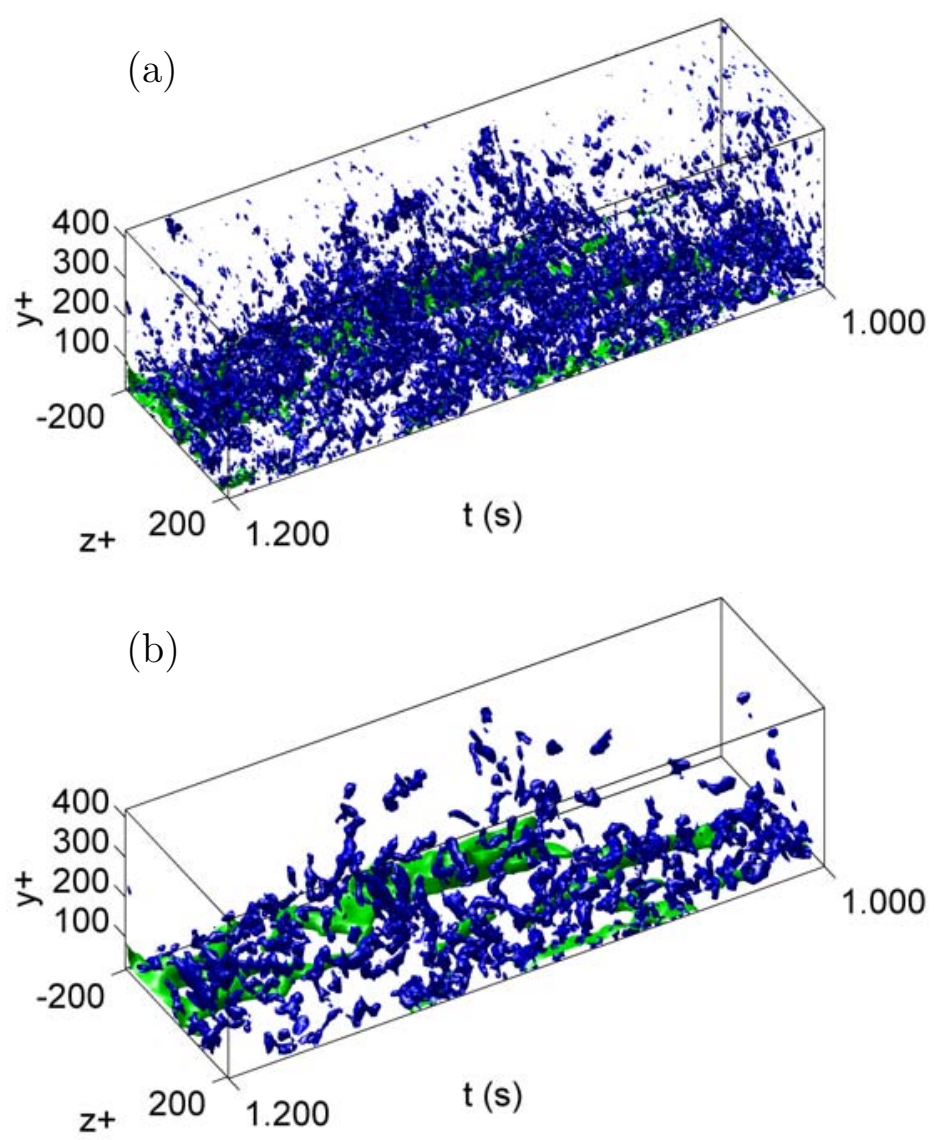

Figure 6.3: Interaction among low-speed streaks (green isosurfaces of $u=1.4 \mathrm{~m} / \mathrm{s}$ ) and turbulent vortical structures (blue isosurfaces of Q-criterion $=8000 \mathrm{~s}^{-1}$ ) inside a turbulent boundary layer from (a) raw and (b) filtered data.

proximity among low-speed regions and vortices was reported by many researchers ([Robinson, 1991b, Dennis and Nickels, 2011a]). Low-speed streaks show branching and meandering behaviours. These findings were in agreement with the literature ([Hutchins and Marusic, 2007, Robinson, 1991a, Dennis and Nickels, 2011b]).

Although a $1 \mathrm{kHz}$, time-resolved, tomo-PIV experiment was carried out, the statistics were calculated using 1204 time-independent velocity fields with a time step of $0.01 \mathrm{~s}$ (runs 10,11, 15, 16 and 17). This time interval was necessary to guarantee decorrelation of the captured flow fields, since the focus of the present section was on flow statistics of the boundary layer and not on instantaneous turbulent structures.

Figure 6.4(a) displays the mean streamwise velocity field at the middle of the volume to show the homogeneity along the spanwise direction. Figure 6.4(b) presents the streamwise velocity profile in as a function of the wallnormal direction. Mean wall-normal and spanwise velocity components were negligible and were not plotted. 

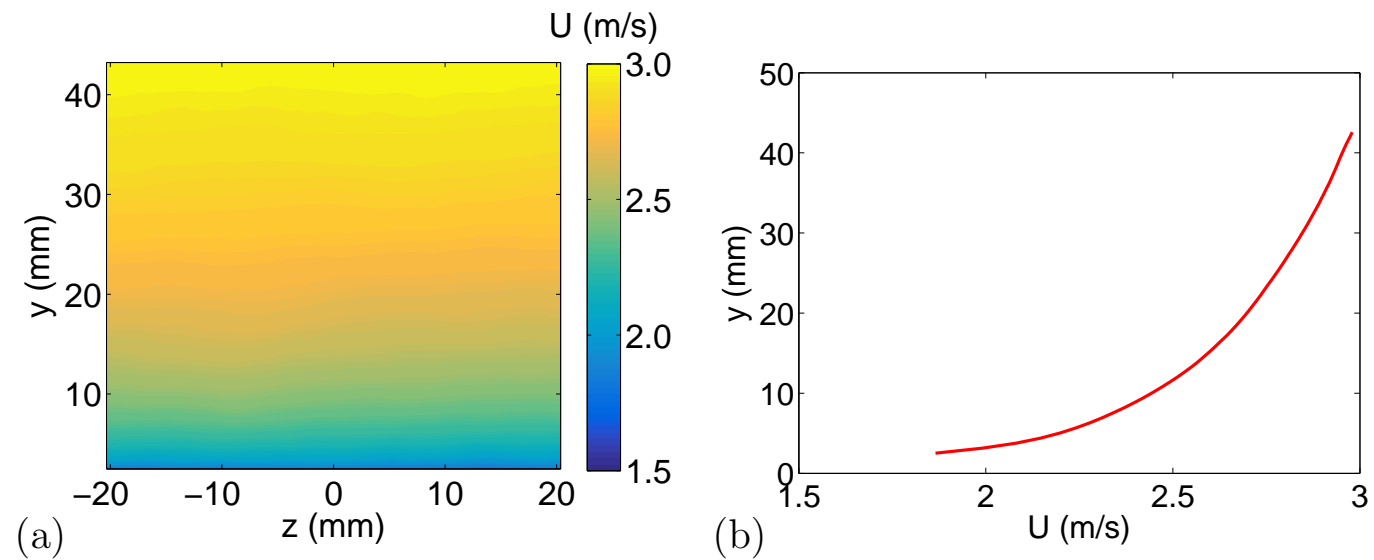

Figure 6.4: (a) Mean streamwise velocity in the middle of the volume $(x=0)$ and (b) profile of streamwise velocity.

Table 6.3: Boundary-layer characteristics.

\begin{tabular}{cccccccc}
\hline$U_{\infty}(\mathrm{m} / \mathrm{s})$ & $\nu\left(\mathrm{m}^{2} / \mathrm{s}\right)$ & $\theta(\mathrm{m})$ & $\delta(\mathrm{m})$ & $u_{\tau}(\mathrm{m} / \mathrm{s})$ & $\delta_{\nu}(\mathrm{m})$ & $R e_{\theta}$ & $R e_{\delta}$ \\
3.6 & $1.50 \times 10^{-5}$ & 0.0354 & 0.300 & 0.147 & $1.00 \times 10^{-4}$ & 8500 & 72000 \\
\hline
\end{tabular}

Table 6.3 summarizes the boundary-layer properties of the present windtunnel experiment, such as free stream velocity $\left(U_{\infty}\right)$, kinematic viscosity $(\nu)$, momentum thickness $(\theta)$, boundary-layer thickness $(\delta)$, friction velocity $\left(u_{\tau}\right)$, viscous length scale $\left(\delta_{\nu}\right)$ and Reynolds numbers based on the momentum thickness $\left(R e_{\theta}\right)$ and on the boundary-layer thickness $\left(R e_{\delta}\right)$. The friction velocity was estimated from a Clauser plot of the velocity profile inside the log region ([Stanislas et al., 2008]), using the von Kármán empirical constants $\kappa=0.41$ and $C=5$.

The uncertainty on velocity measurements was estimated based on the root-mean-square of the divergence of the velocity field given by eq. (3.3.9). Figure 6.5 displays the profile of the root-mean-square of the divergence in relation to the distance from the wall. Horizontal axis is in wall units. The right axis shows the equivalent velocity uncertainty. Higher uncertainty appears near the wall where higher shear and lower velocities were present. In addition, the particle images closer to the wall suffered with light reflections, which increased the noise in the velocity field. The root-mean-square of the velocity divergence (computed within the entire volume) was $67 \mathrm{~s}^{-1}$ leading to a mean uncertainty in velocity, $\delta u^{\prime}$, of the order of 0.15 voxels (equivalent to $0.036 \mathrm{~m} / \mathrm{s}$ or 0.25 wall units). This value of uncertainty was about $10 \%$ of the maximum $u_{\mathrm{rms}}^{\prime}$ and $1 \%$ of the free stream velocity. The present uncertainty result was considered a low level of uncertainty for a tomo-PIV air experiment, and was probably achieved due to the measurements performed in a narrow volume employing 6 cameras. This uncertainty level was comparable to well-controlled experiments found in 


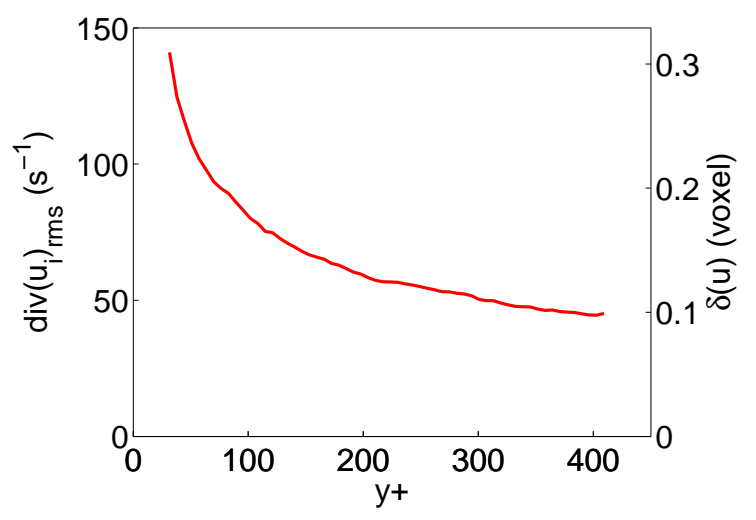

Figure 6.5: Profiles of divergence and uncertainty in velocity field in relation to the distance to the wall for the tomo-PIV boundary-layer experiment.
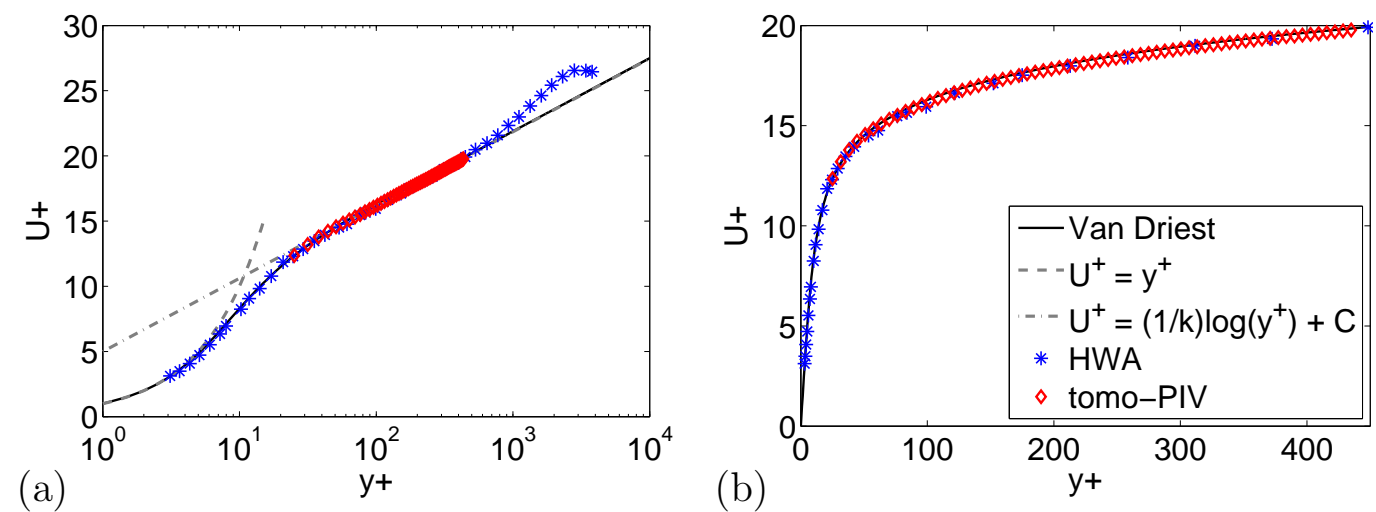

Figure 6.6: Profiles of mean streamwise velocity in (a) semi-log and (b) linear plots.

the literature ([Scarano and Poelma, 2009, Atkinson et al., 2011]).

Figure 6.6 compares velocity statistics from the present tomographic PIV reconstruction with hot-wire anemometer data (HWA) obtained in the same wind tunnel for a boundary layer with $R_{\theta}=8170$ ([Carlier and Stanislas, 2005]). The plot is scaled by inner variables. From this figure, it can be verified that the tomo-PIV and HWA velocity profiles are virtually identical and perfectly agree with the Van Driest profile ([Carlier and Stanislas, 2005]) and log-law.

Good agreement between tomo-PIV and HWA data is also observed in figure 6.7(a) for the profiles of turbulent fluctuating velocity components. Deviations in the measurements from the two techniques are larger near the wall due to the presence of light reflections. It should be mentioned that the measurements of spanwise fluctuating velocity near the wall for the hot-wire are overestimated due to the size of the probe compared to the mean gradient at this region. For comparison, the direct numerical simulations (DNS) of a channel flow with $R e_{\tau}$ of 2000 of [Hoyas and Jiménez, 2008] is also presented. The tomo-PIV experimental data overlap with DNS curves considering the 

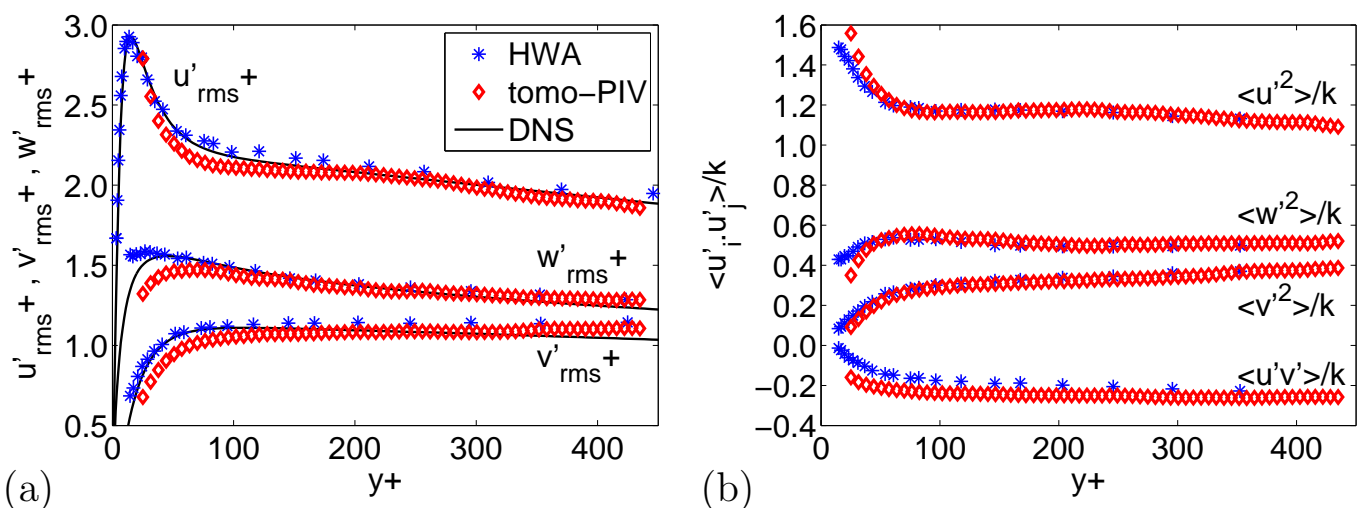

Figure 6.7: Profiles of turbulent velocity fluctuations made dimensionless by (a) wall-units and by (b) the mean kinetic energy. Comparison with hot-wire anemometer measurements (HWA) and DNS of [Hoyas and Jiménez, 2008].

estimated uncertainties. The agreement found in the measurements shown in figure 6.7 is an indication that the recording time was sufficient to obtain converged statistics.

Figure 6.7(b) presents profiles of turbulent velocity fluctuations normalized by the mean kinetic energy for HWA and tomo-PIV data. Good agreement is also observed between the two experimental methods, except for the Reynolds stress $<u^{\prime} v^{\prime}>/ k$.

Profiles of Reynolds stress, $-<u^{\prime} v^{\prime}>/ u_{\tau}^{2}$, and main viscous stress, $(\partial U / \partial y)\left(\nu / u_{\tau}^{2}\right)$, obtained from tomographic PIV and hot-wire anemometer measurements ([Carlier and Stanislas, 2005]), are presented in figure 6.8. Reynolds stress from DNS study of [Hoyas and Jiménez, 2008] is also plotted for comparison. A remarkable agreement among tomo-PIV measurements, DNS and the Van Driest profiles ([Herpin et al., 2013]) can be verified in this figure. The Reynolds stress measured by the HWA deviates from other curves due to calibration problems identified during the post-processing phase of the experiments.

Since tomo-PIV gives access to the full velocity gradient tensor, the rate of dissipation of turbulence kinetic energy by viscous stresses can be calculated by eq. (2.1.8). Figure 6.9 compares the mean dissipation rates, $\epsilon^{+}=\epsilon\left(\nu / u_{\tau}^{4}\right)$, from tomo-PIV and HWA for the boundary-layer data with $R e_{\theta}=8170$ ([Stanislas et al., 2008]) computed with the assumption of Taylor's hypothesis ([Hinze, 1975]) and local isotropic turbulence ([George, 2013]). The dissipation rate from direct numeric simulation of [Hoyas and Jiménez, 2008] is also plotted for comparison. The correlation volume size used to compute the velocity field in the tomo-PIV corresponded to $26 \times 26 \times 26$ wall units, which was equivalent to approximately $8 \eta_{k}$ (Kolmogorov length scale) in each direction (considering $\eta_{k} \approx 3$ wall units of [Carlier and Stanislas, 2005]). Given that 

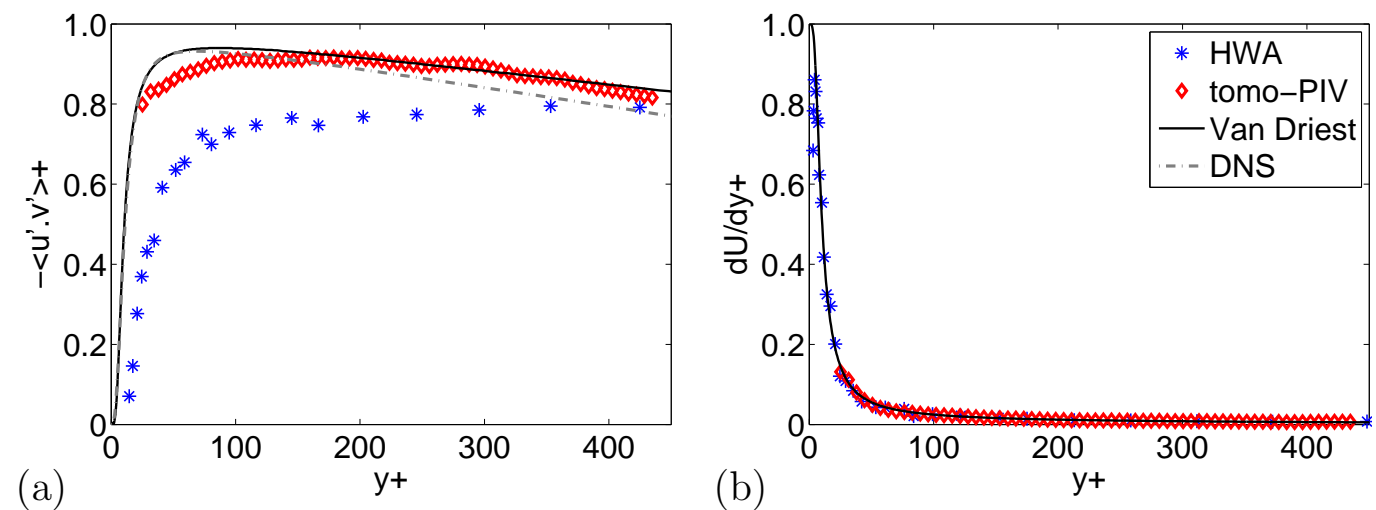

Figure 6.8: Profiles of (a) Reynolds stress and (b) viscous stress. Comparison with hot-wire anemometer measurements (HWA), Van Driest profile and DNS of [Hoyas and Jiménez, 2008].

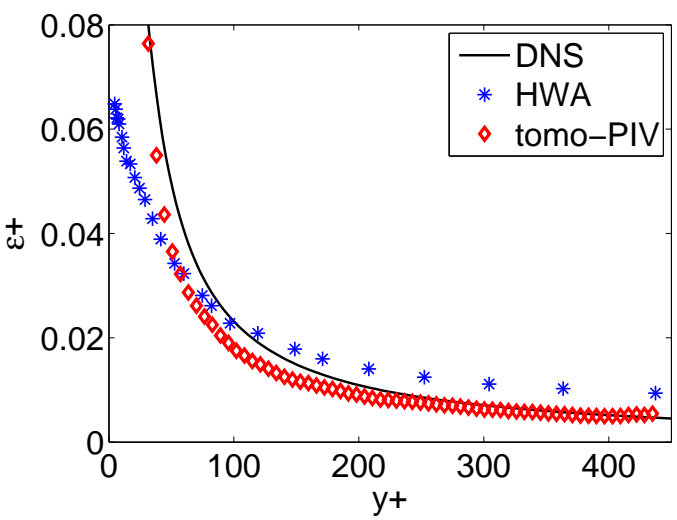

Figure 6.9: Profiles of dissipation for tomo-PIV, hot-wire anemometer measurements (HWA) and DNS of [Hoyas and Jiménez, 2008] in relation to the distance to the wall.

the diameters of the most intense vortical structures were reported to be around 6 to $10 \eta_{k}$ ([Jiménez et al., 1993, Stanislas et al., 2008, Herpin et al., 2013]), it is conceivable that those small vortices were filtered out by the correlation procedure. So, it is expected that the dissipation rate should be marginally underestimated. On top of that, it is important to mention that the computation of dissipation rate from the tomo-PIV data carried a considerable level of uncertainty, mainly due to spatial derivatives that are known to add noise to the results. A good agreement between tomo-PIV measurements and DNS data is observed. It is seen that the HWA measurements deviated from tomo-PIV and DNS results, for positions $y+<100$.

Taking advantage of the full velocity gradient tensor provided by tomo-PIV measurements, all components of vorticity can be computed. Figure 6.10 shows the root-mean-square of fluctuating vorticity components from tomo-PIV measurements and DNS study of [Hoyas and Jiménez, 2008]. The plot is scaled by inner variables. No filtering was employed. The fluctuating 


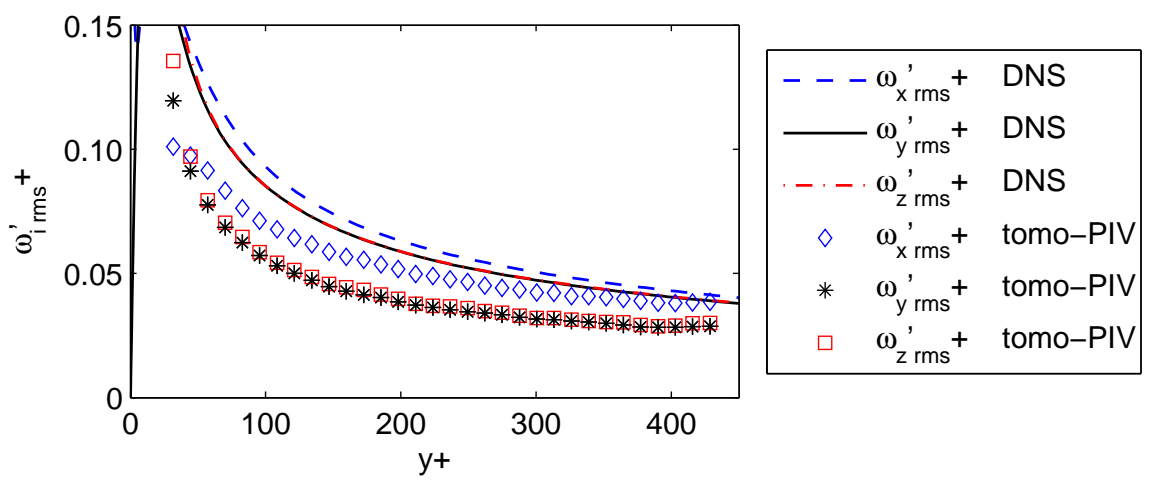

Figure 6.10: Profiles of root-mean-square of fluctuating vorticity components. Comparison of tomo-PIV boundary-layer data with DNS of [Hoyas and Jiménez, 2008]. One over two points are plotted for clarity.

vorticity components of both methods agree considering the uncertainty levels. As already mentioned, the computation of derivatives was contaminated by noise. Once there is not yet a consensus about the estimation of this noise and other sources of errors ([Sciacchitano et al., 2014, Wieneke and Sciacchitano, $2015]$ ), the uncertainties of vorticity components were roughly estimated as being $\delta \omega_{i}^{\prime+} \approx\left(\nu / u_{\tau}\right)\left(\delta u / \triangle_{v e c}\right) \approx 0.04$ adimensionalized by wall variables.

The Kolmogorov length scale, representative of dissipation eddies in turbulent flows, was computed for tomo-PIV data with the dissipation rate (displayed in figure 6.9) and the kinematic viscosity according to eq. (2.1.9). Figure 6.11 presents the profiles of Kolmogorov length scale with respect to the wall-normal distance. At the right axis, the Kolmogorov length scale is made non-dimensionalised by inner variables. Kolmogorov length values increases with wall distance, as expected. The Kolmogorov length scale computed at 60 wall units from the HWA boundary-layer data with $R e_{\theta}=8170$ ([Carlier and Stanislas, 2005]), assuming Taylor's hypothesis and local isotropy, was about $0.3 \mathrm{~mm}$. This HWA value agrees with the present tomo-PIV estimated Kolmogorov length considering the uncertainty level associated.

The Taylor micro-scale of the boundary-layer tomo-PIV experiment was calculated by the osculating parabola from the autocorrelation function of the fluctuating streamwise velocity. Figure 6.12(a) presents the autocorrelation coefficient $R_{u u}$ for positive spatial lags, transformed employing Taylor's hypothesis, computed at $y+=100 . U=U\left(y^{+}\right)$refers to the mean streamwise velocity at the correspondent wall-normal position. The osculating parabola, which its intersection with the abscissa gives the Taylor micro-scale value, is plotted as a red dashed line. Figure 6.12(b) shows the Taylor micro-scale computed by the aforementioned procedure with respect to wall-normal positions. The Taylor length computed by the HWA boundary-layer data for $R e_{\theta}=8170$ of [Carlier 


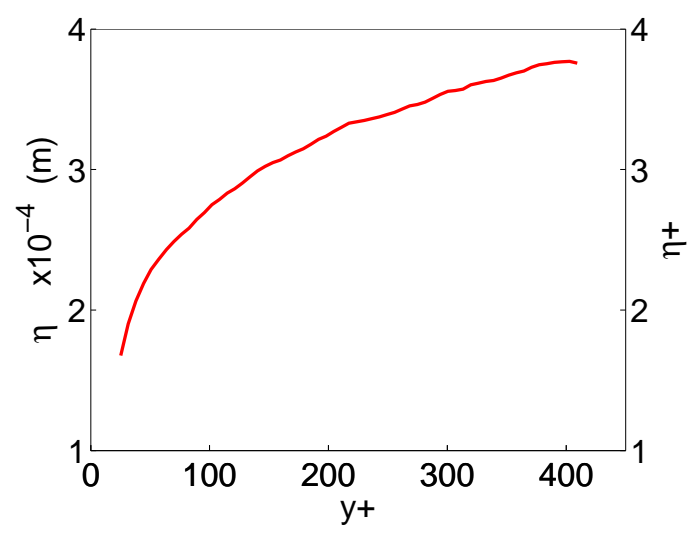

Figure 6.11: Kolmogorov length scale in the tomo-PIV boundary-layer experiment plotted with respect to the wall distance.
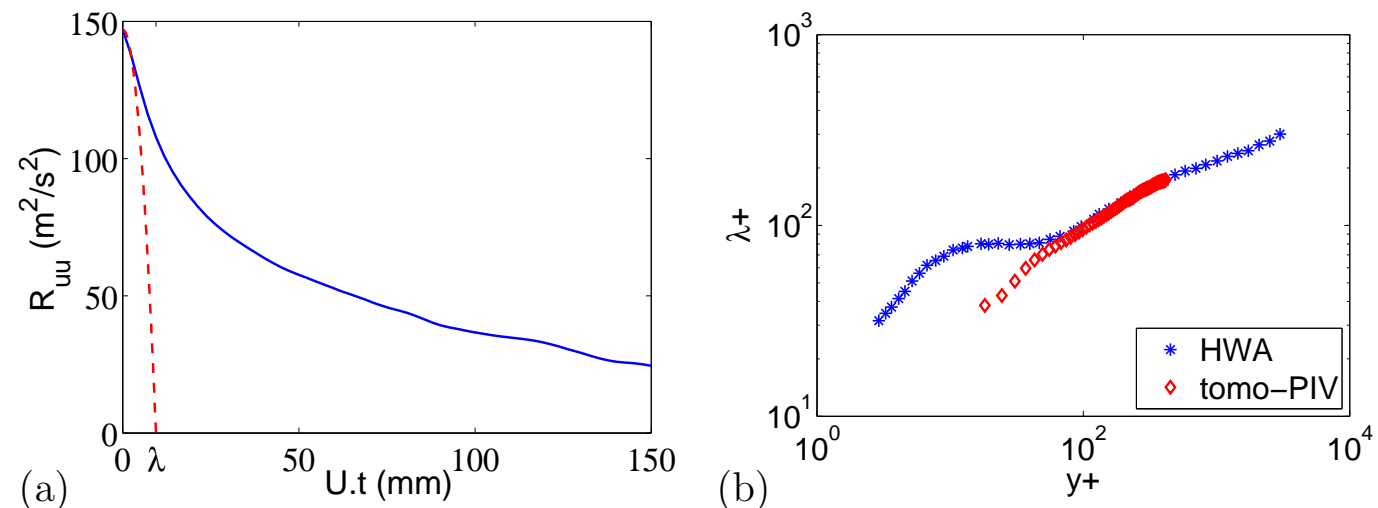

Figure 6.12: (a) Autocorrelation coefficient of streamwise velocity for positive spatial lags (continuous blue line) together with its osculating parabola (red dashed line) showing the Taylor micro-scale at $y+=100$. (b) Taylor microscale computed by the autocorrelation of streamwise velocity for different wallnormal positions for tomo-PIV and HWA techniques.

and Stanislas, 2005] is also plotted for comparison. The two curves overlap for positions above $y^{+}=100$. In the "buffer layer" (around $10<y^{+}<100$ ) the constant behaviour of this micro scale computed by the HWA is not followed by the tomo-PIV measurements. This fact seems to be caused by the size of the interrogation volume employed in the cross-correlation process to compute the velocity fields that do not satisfactorily resolve the small scales, filtering the data. Moreover the tomo-PIV data can display a higher level of noise than the HWA data, which is normally not correlated, and thus affect the first point of $R_{u u}$ employed to fit the parabola. Differently of the HWA, the Taylor length scale from tomo-PIV data displayed a monotonic increase with wall distance for the entire investigated domain. Although the 3D-3C tomo-PIV measurements allows access to Taylor scales along the three directions, only the streamwise micro scale is discussed in the present work.

Taylor micro-scale values can also be estimated by eq. (2.1.10), assuming 


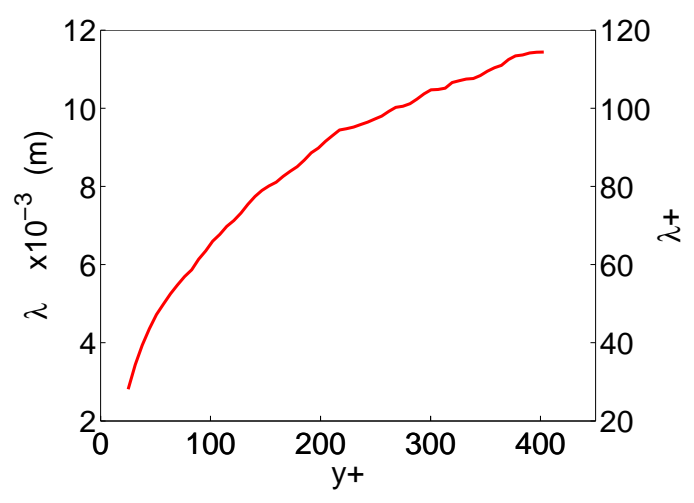

Figure 6.13: Taylor length scales computed by the local isotropic turbulence hypothesis with respect to the distance to the wall from tomo-PIV experimental data.

local isotropic turbulence. Figure 6.13 displays the profiles of Taylor microscale computed by this method. The right axis displays the Taylor micro-scale made dimensionless by inner variables. Despite the fact that the hypothesis of local isotropic turbulence is not recommended for near-wall boundary layer, Taylor micro-scale were computed based on this assumption for comparison. These values were below the Taylor micro-scale values calculated from the autocorrelation function of the fluctuating streamwise velocity (figure 6.12). Nevertheless, Taylor length computed by both methods increased with wall distance, following the same trend of Kolmogorov scale curve (figure 6.11).

Figure 6.14 compares the probability densities function (PDF) of the three velocity components calculated at 50 wall units by tomo-PIV and HWA. A general observation of the distributions for the three velocity components shows excellent agreement between tomo-PIV and HWA data. A small discrepancy is observed for $v^{\prime} / \sqrt{\left\langle v^{\prime 2}\right\rangle}$ and $w^{\prime} / \sqrt{\left\langle w^{\prime 2}>\right.}$ measured by the two techniques probably due to a slight difference in the HWA and tomo-PIV experimental conditions as the data were collected at distinct moments. Fig. 6.14(c) showed a marginal asymmetry in the tomo-PIV that, seemingly, was caused by a little reminiscence of the peak-locking effect, since the deformation method was not applied to the interrogation volume during the correlation process ([Scarano, 2013]), but only sub-pixel shift.

Figure 6.15 presents the power spectral density of the streamwise fluctuating velocity component of the present boundary-layer experiment at a wall-normal position of 100 wall units. The time-resolved velocity data were divided into 512 -size windows with $50 \%$ of overlap before computing the Fast Fourier Transform (FFT) in order to smooth the results. A specific treatment was employed on the FFT velocity windows to impose an artificial periodicity ([Foucaut et al., 2004]). In figure 6.15(b), the power spectral density is 


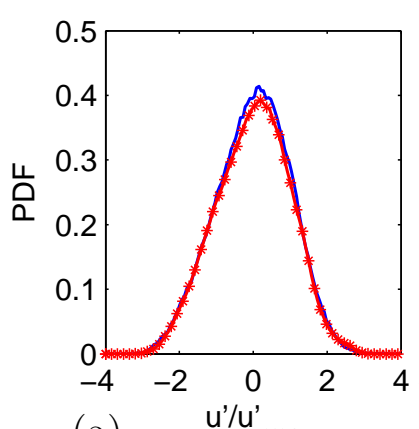

(a)

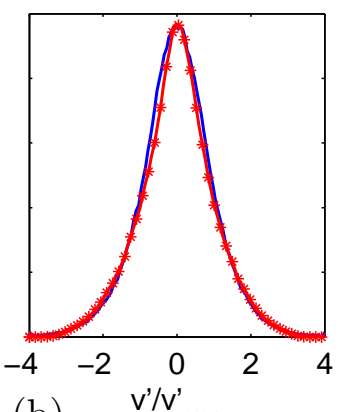

(b) $\quad v^{\prime} / v_{r m s}^{\prime}$

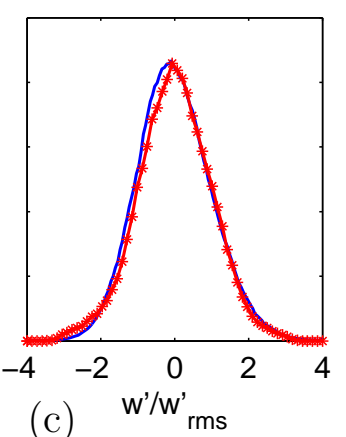

(c) $w^{\prime} / w_{r m s}^{\prime}$

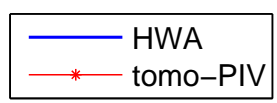

Figure 6.14: Probability density functions at $y^{+}=50$ for (a) $u^{\prime} / \sqrt{<u^{\prime 2}>}$, (b) $v^{\prime} / \sqrt{<v^{\prime 2}>}$ and (c) $w^{\prime} / \sqrt{<w^{\prime 2}>}$.
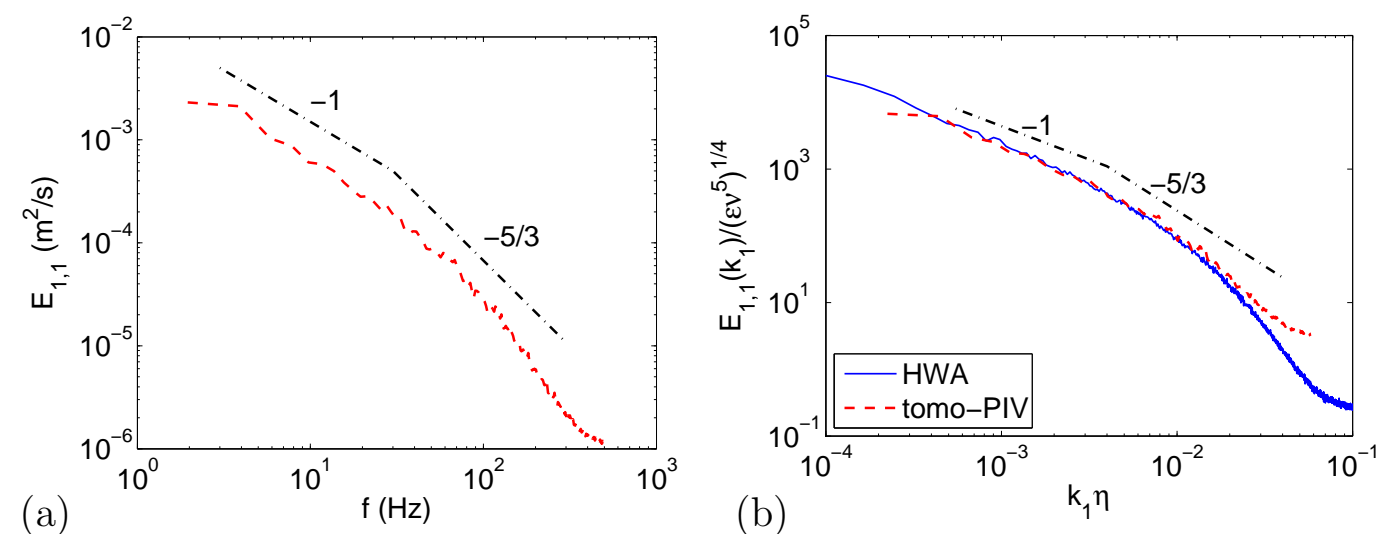

Figure 6.15: Power spectral density for the tomo-PIV streamwise fluctuation velocity component at 100 wall units as a function of (a) frequency and (b) the inverse of Kolmogorov length scale.

made dimensionless by the Kolmogorov length scale (according to [Perry et al., 1986]). The power spectral density of the hot-wire measurements for the same wind tunnel of [Carlier and Stanislas, 2005] are also plotted for comparison. It is possible to observe a region displaying a slope close to -1 (related to energy-containing scale), another region of about $-5 / 3$ slope (related to the inertial range) and a stiffer slope region (related to dissipation). The inflection of the spectrum curve at high wavenumber gives an idea of the noise level. The tomo-PIV curve coincides with that of the HWA, up to a certain value of $k_{1}$. Beyond that value, the noise associated with the tomo-PIV measurements of the present work exceed that of the HWA. This is an expected result if one considers the size of the interrogation volume employed in the cross-correlation procedure.

\subsubsection{Summary of Overall Flow Statistics}

A tomo-PIV technique with six high-speed cameras was applied to study the unsteady character of the near-wall boundary layer flow in a wind-tunnel 
for a momentum-thickness Reynolds number of 8500 .

The optimization of the image pre-processing and the volume reconstruction was successfully performed. The vector fields generated from BIMART reconstructed volumes resulted in good spatial resolution with low measurement errors, of the order of 0.15 voxels, which were estimated by the velocityfluctuation divergence.

Instantaneous flow visualization, employing vortex detection based on the Q-criterion, allowed the identification of vortices interacting with low-speed streaks in the wall boundary layer. The majority of vortices appeared neighbouring the low-speed streaks. The vortices displayed a variety of inclinations and presented a wide range of scales. Low-speed streaks showed branching and meandering behaviours. These findings were in agreement with the literature.

Turbulence statistics from over 8600 vector fields were presented. A good agreement between tomographic PIV and hot-wire anemometer data was observed in terms of velocity profiles and turbulent fluctuations. Also, the experimental data matched the classical model curves, which reveals that the recording time was sufficient to obtain enough converged statistics, since the main goal of the present data was to provide time-resolved measurements. Likewise, the tomo-PIV data displayed good agreement with statistics from direct numerical simulation available in the literature.

Since tomo-PIV gives access to the full velocity gradient tensor, the rate of dissipation and the fluctuating vorticity components were calculated. The tomo-PIV dissipation was in agreement with the dissipation rate computed by the HWA data. A good agreement was also observed among the values of root-mean-square of the fluctuating vorticity from tomographic measurements and direct numerical simulation data.

The probability density functions of the velocity fluctuations from the tomo-PIV method were also compared to the hot-wire anemometer measurements, displaying nearly coincident distributions.

The power spectral density was presented revealing a region of -1 slope (related to energy-containing scale), an other of $-5 / 3$ slope (related to the inertial range) and a stiffer slope region (related to dissipation). These findings were in agreement with previous studies in the literature. 


\subsection{Flow Structure Statistics}

\subsubsection{Pattern Recognition}

In order to compute conditional statistics of near-wall coherent structures, pattern recognition criteria were employed to classify the data. In the present study, the turbulent patterns investigated were low- and high-speed regions, ejections, sweeps and vortices. Each type of coherent structure recognition was established according to prior knowledge of the pattern found in the literature on turbulent flows. The pattern recognition procedure was based on the work of [Lin, 2006]. It is composed of the following steps:

1. Definition of a detection function according to the property of the structure.

2. Determination of a binary field by thresholding the detection function field.

3. Use of mathematical morphology techniques to remove noise and improve the quality of the binary field.

4. Cleaning of the binary fields to remove mis-detected objects and noise.

Details of these procedures are explained in the following sections.

\section{Detection function}

The identification of an specific structure (or object) inside a multidimensional field was implemented by a detection function $F_{d}$, which described the characteristics of this structure. Over the years, besides visualization methods, many eduction techniques to identify these structures and quantify their importance have been proposed based on quadrant analyses ([Wallace et al., 1972]), variable interval averaging (VITA) ([Blackwelder and Kaplan, 1976]), proper orthogonal decomposition (POD) ([Holmes et al., 1998]), pressure ([Robinson, 1991b]) and various invariants of the velocity-gradient tensor ([Jeong et al., 1997]). The function is defined according to the property of the structure, which is inferred from the observations in the experiments or numerical simulations, and is expressed as

$$
F_{d}=f\left(\operatorname{var}_{1}, \operatorname{var}_{2}, \ldots, \operatorname{var}_{n}\right)
$$

where $v a r_{1}, v a r_{2}, \ldots, v a r_{n}$ are variables characterising the structure to be detected. 
In the present study, the detection function $F_{d}$ was applied to the tomo-PIV velocity field data according to the turbulent structure to be analysed. Details of these functions will be described in the specific turbulent structure section.

\section{Binary field}

Binary fields are obtained after thresholding a detection function field. Thresholding is a standard procedure in digital image processing, in which 2D grey intensity images are transformed into binary images, which have only two possible values 1 or 0 ([Gonzalez, 2009]). The procedure of thresholding can be easily extended to multidimensional matrices.

In the matrix $F_{B}$, each element can be converted to 1 if its detection function value $F_{d}$ is higher than or equal to the threshold level $F_{\text {thresh }}$, and to 0 if it is lower than the threshold. Another possibility is to transform to 1 if the detection function value is lower than or equal to the threshold level, and to 0 otherwise.

$$
\begin{aligned}
& F_{B}= \begin{cases}1 & \text { if } F_{d} \geq F_{\text {thresh }} \\
0 & \text { otherwise }\end{cases} \\
& F_{B}= \begin{cases}1 & \text { if } F_{d} \leq F_{\text {thresh }} \\
0 & \text { otherwise }\end{cases}
\end{aligned}
$$

Additional detection functions can be used simultaneously to produce the binary field.

$$
F_{B}= \begin{cases}1 & \text { if } \quad F_{d}^{a} \geq F_{\text {thresh }}^{a} \text { and } F_{d}^{b} \geq F_{\text {thresh }}^{b} \text { and } \ldots \text { and } F_{d}^{z} \geq F_{\text {thresh }}^{z} \\ 0 & \text { otherwise }\end{cases}
$$

Although the tomo-PIV field produces a 3D discrete domain, in the present work, only the middle plane (2D field) was used to produce conditional statistics. This procedure was adopted since the thin volume employed did not allow the extraction of spatial features of the structure along the streamwise direction. Nevertheless, the 3D data is very useful to compute all spatial velocity gradients and to aid in the filtering of noise by using the values of neighbours.

Note that spatial characteristics along the streamwise axis in timeresolved data could be extracted, which, however, was not the aim of the present work. In such a case, the time could be transformed into space multiplying it by the local mean velocity according to Taylor's hypothesis ([Hinze, 1975]). This procedure is controversial since it assumes that structures do not change when they are convected, which normally is not true ([Moin, 
2009, Ganapathisubramani, 2013]). In addition, converting time into space would require an interpolating grid because the average streamwise velocity varies along the wall-normal direction.

\section{Mathematical morphology operations}

In order to remove noise and to improve the quality of the binary field $F_{B}$, obtained by the thresholding procedure, mathematical morphology operations were employed.

Erosion and dilatation are classical morphological operations. The dilatation of a set $A$ by a structuring element $B$ is the Minkowski addition of $A$ and $B$ given by ([Gonzalez, 2009]):

$$
D_{B}(A)=A \oplus B=\{(x+y) \mid x \in A, y \in B\}
$$

An alternative definition is given by the union of copies of the structuring element $B$ centred at every location $x$ in the foreground.

Similarly, the erosion is the Minkowski subtraction of $A$ and $B$.

$$
E_{B}(A)=A \ominus B=\{x \mid(x+y) \in A \forall y \in B\}
$$

In other words, only positions $x \in A$ such that when the structuring element $B$ centred at $x$ fits within $A$, remain.

In practice, the set $A$ will be the input binary data set created by the thresholding procedure, which has values 1 for the structures and 0 for the background. The set $B$ is a binary structuring element defined as any shape according to the application.

Erosion generally decreases object sizes and removes small anomalies by eliminating objects that are smaller than the structuring element. Dilation generally increases object sizes, filling holes and broken regions, and connecting objects that are separated by spaces smaller than the size of the structuring element. Figures 6.16 and 6.17 show examples of dilation and erosion operations applied on binary fields.

\begin{tabular}{|l|l|l|l|l|}
\hline 0 & 0 & 0 & 0 & 0 \\
\hline 0 & 1 & 1 & 1 & 0 \\
\hline 0 & 1 & 0 & 1 & 0 \\
\hline 0 & 1 & 1 & 1 & 0 \\
\hline 0 & 0 & 0 & 0 & 0 \\
\hline
\end{tabular}

\begin{tabular}{|l|l|l|}
\hline 0 & 1 & 0 \\
\hline 1 & 1 & 1 \\
\hline 0 & 1 & 0 \\
\hline
\end{tabular}

\begin{tabular}{|l|l|l|l|l|}
\hline 0 & 1 & 1 & 1 & 0 \\
\hline 1 & 1 & 1 & 1 & 1 \\
\hline 1 & 1 & 1 & 1 & 1 \\
\hline 1 & 1 & 1 & 1 & 1 \\
\hline 0 & 1 & 1 & 1 & 0 \\
\hline
\end{tabular}

Figure 6.16: Dilation operation of a square object with a hole in the middle by a diamond structuring element. The result is a larger object without the hole. 


\begin{tabular}{|l|l|l|l|l|}
\hline 0 & 0 & 0 & 0 & 1 \\
\hline 0 & 0 & 0 & 0 & 0 \\
\hline 1 & 1 & 1 & 1 & 0 \\
\hline 1 & 1 & 1 & 1 & 0 \\
\hline 1 & 1 & 1 & 1 & 0 \\
\hline
\end{tabular}$\quad \ominus \quad$\begin{tabular}{|l|l|l|}
\hline 0 & 1 & 0 \\
\hline 1 & 1 & 1 \\
\hline 0 & 1 & 0 \\
\hline 0
\end{tabular}$=$\begin{tabular}{|l|l|l|l|l|}
0 & 0 & 0 & 0 & 0 \\
\hline 0 & 0 & 0 & 0 & 0 \\
\hline 0 & 0 & 0 & 0 & 0 \\
\hline 1 & 1 & 1 & 0 & 0 \\
\hline 1 & 1 & 1 & 0 & 0 \\
\hline
\end{tabular}

Figure 6.17: Erosion operation of two objects by a diamond structuring element. The result is a smaller bottom-left object and the suppression of the upper-right small object.

catchment basins begin watershed line

filling with water forms here
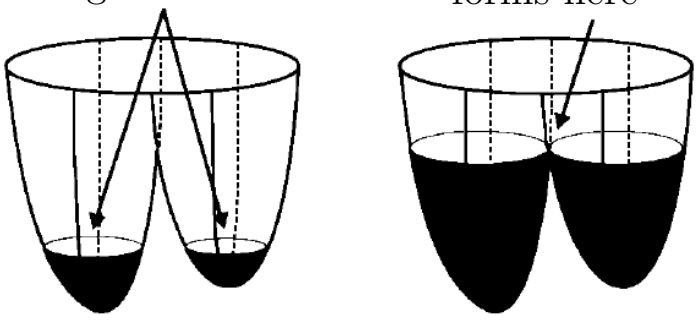

Figure 6.18: Watershed segmentation proceeds by computationally immersing the underside region as a catchment basin (from [Rettmann et al., 2000]).

Single dilation or erosion expands or reduces object sizes resulting in geometric distortion. To avoid this effect, dilation and erosion operations are commonly combined together into closing and opening operations. The morphological operation called closing is a dilation followed by an erosion, while the opening is an erosion followed by a dilation. These mathematical morphology tools can be used in an interactive way to smooth noise and improve the shape of the objects.

The watershed algorithm ([Roerdink and Meijster, 2000]) can be employed in order to split large structures or to disconnect different structures wrongly classified as being a larger structure. The term watershed refers to a ridge that divides regions drained by different river systems and the geographical area draining into a river or reservoir is called catchment basin. Generally, any $2 \mathrm{D}$ matrix with real values can be considered as a topographic surface. If this surface is flooded from its global minima and if the merging of the waters coming from different sources are not allowed to mix, the matrix can be partitioned into catchment basins and watershed lines, as sketched in figure 6.18.

The mathematical description of the watershed transform by simulated immersion can be found in the work of [Roerdink and Meijster, 2000]. Consider 
$f: D \rightarrow \Re$ as being a matrix of real numbers, with $h_{\min }$ and $h_{\max }$ as the minimum and maximum value of $f$. Define a recursion with the matrix intensity level $h$ increasing from $h_{\min }$ to $h_{\max }$, in which the basins associated with the minima of $f$ are successively expanded. Let $X_{h}$ denote the union of the set of basins computed at level $h$. A connected component of the threshold set $T_{h+1}=\{p \in D \mid f(p) \leq h+1\}$ at level $h+1$ can be either a new minimum, or an extension of a basin in $X_{h}$. In the latter case, the geodesic influence zone of $X_{h}$ within $T_{h+1}$ is computed, resulting in an update $X_{h+1}$. Let $\min _{h}$ denote the union of all regional minima at altitude $h$. The watershed recursion can be expressed as

$$
\left\{\begin{array}{l}
X_{h_{\min }}=\left\{p \in D \mid f(p)=h_{\min }\right\}=T_{h_{\min }} \\
X_{h+1}=\min _{h+1} \cup I Z_{T_{h+1}}\left(X_{h}\right), \quad h \in\left[h_{\min }, h_{\max }\right)
\end{array}\right.
$$

where $I Z_{T}(X)$ is the union of the geodesic influence zones of $k$ partitions of the connected components of $X$ given by

$$
I Z_{T}(X)=\bigcup_{i=1}^{k}\left\{p \in T \mid \forall j \in[1 \ldots k] \backslash\{i\}: d_{T}\left(p, X_{i}\right)<d_{T}\left(p, X_{j}\right)\right\}
$$

being $d_{T}(p, X)=\min _{q \in X}\left(d_{T}(p, q)\right)$ the minimum path length among all paths from $p$ to $q$ within $T$.

The watershed $W(f)$ of $f$ is the complement of $X_{h_{\max }}$ in $D$ :

$$
W(f)=D \backslash X_{h_{\max }}
$$

For an example of watershed transform according to the above recurrence, see figure 6.19. In this figure, extracted from [Roerdink and Meijster, 2000], $A$ and $B$ are labels of basins and $W$ is used to denote watershed. The global minima in the input matrix, equal to zero, are indicated in bold. The process starts with $h$ equal to the matrix minimum. The $h$ value is gradually increased till the maximum of the matrix. The watershed algorithm prevents the basins $\mathrm{A}$ and $\mathrm{B}$ to join.

\begin{tabular}{|l|l|l|}
\hline 3 & 2 & 2 \\
\hline 3 & 1 & 1 \\
\hline $\mathbf{0}$ & 1 & $\mathbf{0}$ \\
\hline
\end{tabular}

(a)

\begin{tabular}{|l|l|l|}
\hline 3 & 2 & 2 \\
\hline 3 & 1 & 1 \\
\hline $\mathrm{A}$ & 1 & $\mathrm{~B}$ \\
\hline
\end{tabular}

(b) $h=0$

\begin{tabular}{|c|c|c|}
\hline 3 & 2 & 2 \\
\hline 3 & W & B \\
\hline A & W & B \\
\hline
\end{tabular}

(c) $h=1$

\begin{tabular}{|c|c|c|}
\hline 3 & B & B \\
\hline 3 & B & B \\
\hline A & W & B \\
\hline
\end{tabular}

(d) $h=2$

\begin{tabular}{|c|c|c|}
\hline $\mathrm{B}$ & $\mathrm{B}$ & $\mathrm{B}$ \\
\hline $\mathrm{W}$ & $\mathrm{B}$ & $\mathrm{B}$ \\
\hline $\mathrm{A}$ & $\mathrm{W}$ & $\mathrm{B}$ \\
\hline
\end{tabular}

(e) $h=3$

Figure 6.19: Watershed transform by immersion on the 4-connected grid, showing relabelling of watershed positions. (a) Original image; (b-e) labelling steps based on eq. (6.2.7) (from [Roerdink and Meijster, 2000]). 
In the present binary fields, a closing operation was employed to connect separated objects and to fill holes. Then an opening operation was applied to remove isolated small objects, sharp peaks or capes. The structuring element had a diamond shape of size $3 \times 3$. The structuring element was chosen according to the shape of objects expected in the wall-normal-spanwise plane. Large structuring elements are not used once they can connect several separated coherent structures or cut into several small pieces others, depending on structure patterns, or eliminate small structures. The watershed algorithm was employed in the conditional statistics of low- and high speed regions, ejections and sweeps to split large structures.

\section{Cleaning procedure}

After the mathematical morphology procedure, many small objects, which are larger than the structuring element used, but not enough to be considered as coherent structures, remain in the binary field $F_{B}$. These objects should be filtered out before statistical analysis is applied. Therefore, a cleaning procedure to remove objects that have an area $A_{i}$ smaller than a threshold $A_{\text {thresh }}$ can be adopted as in the following equation.

$$
F_{B}= \begin{cases}0 & \text { if } A_{i} \leq A_{\text {thresh }} \\ 1 & \text { otherwise }\end{cases}
$$

\section{Features of the coherent structures}

For each connected region in the binary field, the tomo-PIV measurements of velocity components and its derived quantities, such as fluctuating velocity components, vorticity components, fluctuating vorticity components, Reynolds stress components, dissipation rate, to mention a few examples, can be assessed. These physical quantities can be averaged within the connected structure, or can be obtained from a specific position (e.g., centroid, local maximum of detection function or local minimum of detection function). In addition to the mentioned quantities, the geometric features, as size of the bounding box of the structure, centroid, volume (or area in 2D fields), spanwise spacing between structures, among others, can also be extracted directly from the binary field. These coherent structures features allow for conditional sampling statistics. Turbulent structures can be grouped around the same wallnormal positions to construct probability density functions, averages and rootmean-squares.

In this work, the conditional arithmetic mean of each wall-normal position was calculated summing the analysed flow quantities of each structure 
nearby the same position and dividing the summation by the amount of structures used in the computation.

$$
<\xi>\left(y_{i}^{+}\right)=\frac{1}{N_{k}} \sum_{k=1}^{N_{k}} \xi_{k},
$$

where $\xi$ represents a flow quantity, $k$ the index of structures nearby the wallnormal position $y_{i}^{+}$and $N_{k}$ the amount of structures at this region.

The conditional root-mean-square calculations were analogous.

$$
\xi_{\mathrm{rms}}\left(y_{i}^{+}\right)=\sqrt{\frac{1}{N_{k}} \sum_{k=1}^{N_{k}} \xi_{k}^{2}}
$$

It should be mention that the conditional statistics defined in the present work delivers values of the flow properties relative to the size of the analysed structures. In order to compute their absolute contribution to the flow, the ratio between structure and flow volumes (or areas in $2 \mathrm{D}$ fields) must be taken into account.

\subsubsection{Low- and High-Speed Regions}

\section{Low- and high-speed region detection and feature extraction}

Low- and high-speed regions were defined as locations of negative and positive fluctuating streamwise velocities, respectively ([Lin, 2006, Kang et al., 2007, Dennis and Nickels, 2011b, Dekou et al., 2016]). Figure 6.20(a) displays the probability density functions $(\mathrm{PDF})$ of the fluctuating streamwise velocities along wall-normal direction. The multi-peak distributions for $y^{+}>80$ seemed to be a result of the insufficient amount of data to converge the current statistics. Apparently, the peak-locking effect was not the cause of these multipeak distributions, since a multi-pass cross-correlation with sub-pixel shift was employed and, in addition, figure 6.14 displayed nice distributions of the three fluctuation velocity components collapsing with the hot-wire anemometer data at $y^{+}=50$. Trying to generalise the detection method to different Reynolds number and to account for possible variations in the width of $u^{\prime}$ distribution along the wall-normal axis, the fluctuating streamwise velocity was normalized by its root-mean-square $u_{\mathrm{rms}}^{\prime}\left(y^{+}\right)$. The PDF variation of the normalized version $u^{\prime} / u_{\mathrm{rms}}^{\prime}\left(y^{+}\right)$are presented in figure 6.20(b). Therefore, in the present study, lowand high-speed region detection function $F_{d}$ was defined as the normalized streamwise fluctuating velocity.

$$
F_{d}=f\left(u^{\prime}\left(x^{+}, y^{+}, z^{+}\right), u_{\mathrm{rms}}^{\prime}\left(y^{+}\right)\right)=\frac{u^{\prime}\left(x^{+}, y^{+}, z^{+}\right)}{u_{\mathrm{rms}}^{\prime}\left(y^{+}\right)},
$$



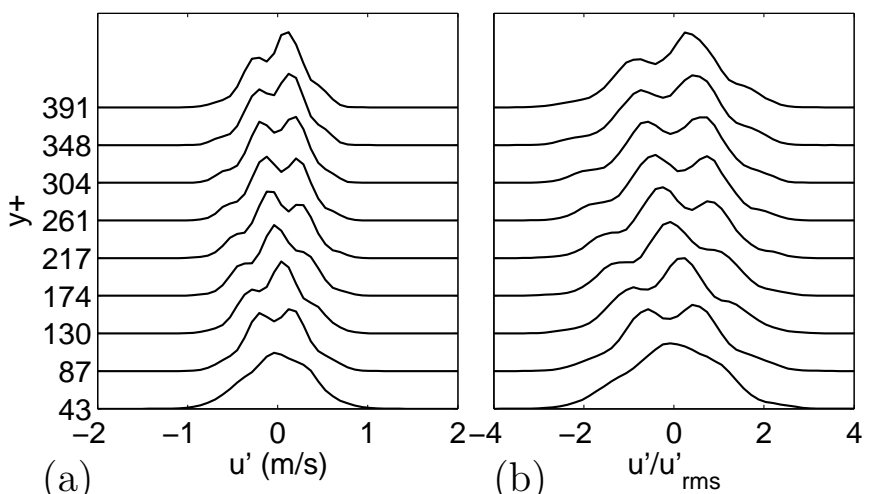

(b)

Figure 6.20: PDF of (a) $u^{\prime}$ and (b) $u^{\prime} / u_{\text {rms }}^{\prime}$ values used to detect low- and high-speed regions for different wall-normal distances.

where the streamwise position $x^{+}$refers to the middle plane $\left(x^{+}=0\right)$.

The above detection function, applied to the tomo-PIV data, is a real scalar field defined on a discrete domain. Low-speed regions were detected applying a negative constant value of thresholds $F_{\text {thresh }}$ in the $F_{d}$ field, while high-speed regions were classified employing positive constant values.

$$
\begin{gathered}
\text { Low-speed region } F_{B}= \begin{cases}1 & \text { if } F_{d} \leq F_{\text {thresh }} \\
0 & \text { otherwise }\end{cases} \\
\text { High-speed region } F_{B}= \begin{cases}1 & \text { if } F_{d} \geq F_{\text {thresh }} \\
0 & \text { otherwise }\end{cases}
\end{gathered}
$$

In the binary field $F_{B}$, the structures are expressed by 1 (white color) and the background by 0 (black color). In the present study, the threshold level to classify low-speed regions was selected as -2 , while the threshold to classify high-speed regions was selected as 2. These threshold values were chosen after various tests and they were based on the balance between the amount of detected turbulent structures and the spurious regions. In the case of high-speed regions, employing a lower threshold value created binary fields composed of fewer amount of large structures. Increasing the threshold value decreased the structure sizes disconnecting them. Over a certain range of threshold values, the general object shape did not modify, only slight changes were observed in the size. However, increasing the threshold above a certain level decreased the influence of background noise. The increment of threshold value beyond the mentioned range, decreased significantly the size and amount of detected structures. For the low-speed detection, analogous behaviour was observed with the inverse threshold variation. These findings were in agreement with [Lin, 2006].

Figure 6.21 exemplifies the detection of low- and high-speed regions by 
the described procedure. The $F_{d}$ field of figure $6.21(\mathrm{a})$, being blue mapped to negative and red to positive values, was thresholded resulting in the binary field $F_{B}$ of figure $6.21(\mathrm{~b})$. This binary field was filtered by closing, opening and cleaning operations producing the field of figure 6.21(c), which contain the low-speed regions (white color). The binary fields were filtered by one iteration of closing and opening operations with a structuring element of a $3 \times 3$ diamond shaped. The cleaning procedure removed structures with areas of about 205 wall units, which is equivalent to 5 grids. This procedure filtered the noise in the structure geometry. Similar procedure was adopted in the same $F_{d}$ field for high-speed regions resulting in the binary field of figure 6.21(d). Figure 6.22 shows the threshold influence in the filtered binary fields of lowspeed regions in the the middle plane of the tomo-PIV data. In these samples, threshold levels $F_{\text {thresh }}$ of $-1,-1.5,2$, and -2.5 were applied on the detection function $F_{d}=u^{\prime} / u_{\text {rms }}^{\prime}$ of figure 6.21(a).

Figure 6.23 shows the frequency of occurrence of the position of low- and high speed region centres $\left(y_{0}\right)$ with respect to the wall distance. The position is scaled by inner variables. The turbulent structures were classified using both detection functions, $u^{\prime}$ and $u^{\prime} / u_{\text {rms }}^{\prime}\left(y^{+}\right)$. Each bar in the plot represents the statistics over all structures that have their centres within a range around a determined wall distance. Both detection functions $F_{d}$ show an almost uniform distributions, except for the borders. The watershed algorithm was employed to split turbulent regions into smaller connected regions in order to improve the convergence of statistical results. Prior testes showed that results using the watershed algorithm were similar to the traditional conditional statistics where the arithmetic average operation is computed within non-zero regions of the binary field and does not take into account if this regions are connected or not. The total amount of detected low-speed regions was about 41000 and high-speed regions was about 45000 .

From each turbulent structure, fluctuating velocity components $\left(u^{\prime}, v^{\prime}\right.$ and $\left.w^{\prime}\right)$, fluctuating vorticity components $\left(\omega_{x}^{\prime}, \omega_{y}^{\prime}\right.$ and $\left.\omega_{z}^{\prime}\right)$, Reynolds stress $\left(-u^{\prime} v^{\prime}\right)$ and dissipation rate $(\epsilon)$ can be averaged within the connected region in the binary field. It is important to mention that, despite the fact that the analysis was done based on planes instead of 3D fields, differently from the standard PIV, tomo-PIV allows assessing all components of velocity and vorticity, 3D Q-criterion and dissipation quantities.

In the present work, geometric characteristics of low- and high-speed structures were not computed because the majority of these type of structures touched field borders. The remainder structures were not sufficient to produce representative conditional statistics of geometric quantities and the use of 
watershed algorithm would not produce realistic statistics of the geometric characteristics since it oversegments the structure (example in figure 6.48).

In addition to the above mentioned quantities, the inclination angle of any individual structure intersecting the measurement plane can be computed based on the orientation of the fluctuating velocity vector. Projection angles were proposed in order to analyse the structure orientation. Figure 6.24 depicts the projection angles with respect to the streamwise-wall-normal plane $\left(\Phi_{x y}\right)$, streamwise-spanwise plane $\left(\Phi_{x z}\right)$ and wall-normal-spanwise plane $\left(\Phi_{y z}\right)$. This figure also presents the related quadrant definition for the projection angles based on the fluctuating velocity.

These projection angles were defined as

$$
\begin{aligned}
& \Phi_{x y}=\arccos \left(\frac{u^{\prime}}{\sqrt{u^{\prime 2}+v^{\prime 2}}}\right) \operatorname{sign}\left(v^{\prime}\right) \\
& \Phi_{x z}=\arccos \left(\frac{u^{\prime}}{\sqrt{u^{\prime 2}+w^{\prime 2}}}\right) \operatorname{sign}\left(w^{\prime}\right) \\
& \Phi_{y z}=\arccos \left(\frac{v^{\prime}}{\sqrt{v^{\prime 2}+w^{\prime 2}}}\right) \operatorname{sign}\left(w^{\prime}\right)
\end{aligned}
$$

The projection angles could assume values between $-180^{\circ}$ and $+180^{\circ}$.

The conditional statistics were computed in relation to structures nearby the same wall-normal position. Turbulent structures that have their centre around the same distance from the wall can be grouped to construct probability density functions, averages and root-mean-squares.

Low- and high speed region detection and feature extraction can be summarised as follows

1. Detection of all regions with normalized fluctuating streamwise velocity $\left(u^{\prime} / u_{\text {rms }}^{\prime}\right)$ lower or higher than a threshold for low- or high-speed regions, respectively.

2. Closing and opening operations to attenuate noise in the structure geometry.

3. Cleaning operation to filter out small connected regions.

4. Extraction of structure features as the average of these features inside the connected region.

5. Computation of conditional statistics of the various features for different wall-normal positions. 


\section{Low- and high-speed region statistics}

Instead of looking for instantaneous turbulent structure organization, this work focus on the structure statistics based on conditional sampling. Despite the interesting qualitative results of figure 6.3, the amount of data processed was not sufficient to provide 3D geometrical conditional analysis of turbulent structures. Because of that, statistical results were obtained using the features of detected structures in the wall-normal-spanwise plane at a fixed axial position $(x=0)$ from 1724 flow fields separated by a time interval of $0.005 \mathrm{~s}$. The same tomo-PIV measurement fields were used for low- and highspeed regions, ejections, sweeps and vortices.

To understand how turbulent structures travel in the flow, the PDF of the fluctuating velocity components within the turbulent structures is presented in figure 6.25 with respect to the distance from the wall. The streamwise and wall-normal fluctuating velocity seem to display log-normal distributions with negative and positive averages, respectively, for low-speed regions. The distributions of the same fluctuating velocity components for highspeed regions look to be mirrored versions of the previous ones, i.e. log-normal distributions with positive and negative averages, respectively. The offset of the stream-wise velocity distributions were directly linked to the threshold employed. Higher absolute value of the threshold $F_{\text {thresh }}$ increased the abscissa distance between the distribution peak and the zero. The spanwise fluctuating velocity displayed more spread distributions centred on zero for both types of turbulent structures. All fluctuating velocity distributions for different wallnormal positions, with the exception of the first ones that were located at the buffer layer, are similar among the correspondent velocity component and structure type. Slight variations were attributed to the amount of turbulent structures used to compute the conditional statistics.

To clarify the behaviour of fluctuating velocity components, the mean was computed with respect to the wall-normal position of the turbulent structures. The conditional statistics were computed in relation to structures nearby the same wall-normal position. These results are presented in figure 6.26 for lowand high-speed regions. Both structures display zero mean in the spanwise fluctuating velocities. The low and high values of the mean stream-wise velocity components for low- and high-speed regions, respectively, were expected due to the definition of these structures based on the stream-wise velocity detection criteria. The first point of the mean stream-wise velocity in figure 6.26(a) seem to be influenced by the high population of low-speed streaks at this wall-normal position ([Kline et al., 1967, Robinson, 1991a]). The low-speed structures present a positive wall-normal velocity component, considering the 
prevailing levels of expected uncertainties $\left(\delta u^{\prime+}=\delta u^{\prime} / u_{\tau} \approx 0.25\right.$ wall units). Consequently, these structures moved, in average, to the upper part of the boundary layer while flowing forward in a slower rate than the surrounding fluid. In the opposite way, the high-speed regions moved, in average, faster than the surrounding flow in the forward direction and toward the wall. These findings were in agreement with [Lin, 2006], which investigated conditional statistics of turbulent structures, employing stereo PIV in streamwise-spanwise planes located at $10<y^{+}<50$, in the same wind tunnel of the present study with $R e_{\theta}=7800$.

Figure 6.27 presents a comparison of the root-mean-square of fluctuating velocity components of low- and high-speed regions and the correspondent velocity components of the overall flow. It is important to recall that these root-mean-square values were computed based on the amount of structures around the wall-normal position. It did not take into account the structure area, neither the relative area in relation to the flow field. So these plots shows the relative value of the flow quantity inside the structures. The turbulent structures analysed display root-mean-square values of the streamwise components higher than the surrounding fluid. These findings were expected due to the removal of low absolute values of fluctuating streamwise velocity during the threshold procedure to classify low- and high-speed regions. All other curves coincide with the global flow statistics. Regarding the contribution of low-speed regions in the total root-mean-square of fluctuating streamwise velocity, it was about $27 \%$ (on average) with a relative structure area of about $23 \%$ of the total flow area, while the contribution of high-speed regions was about $26 \%$ with an area of about $22 \%$ of the total area. The similar contribution to the total root-mean-square of fluctuating streamwise velocity of large-scale lowand high-speed structures was also reported by [Dekou et al., 2016]. [Dekou et al., 2016] analysed the large-scale turbulent structures by Linear Stochastic Estimation to correlate stereo-PIV and hot wires anemometry data from the same wind tunnel with $R e_{\theta}$ around 9800 .

The Reynolds stresses, calculated within the turbulent structures, are presented in figure 6.28(a) with respect to wall-normal position. The Reynolds stress of flow statistics are also plotted in the figure for comparison purposes. The conditional statistics show higher Reynolds stresses within the low- and high-speed regions than the flow statistics considering the estimated uncertainties $\left(\delta u^{\prime} v^{\prime+} \approx \sqrt{2} \delta u^{\prime+} \approx 0.35\right.$ in wall variables). The $-<u^{\prime} v^{\prime}>$ values increase with the decreasing of low-speed threshold and with the increasing of the high-speed threshold. It is interesting to observe that the Reynolds stress curve for low-speed regions appears over the curve corresponding to the 
high-speed region. This finding does not seem to be a result of the threshold value employed, and it was reported by many researchers (e.g., [Talmon et al., 1986, Kang et al., 2007, Dennis and Nickels, 2011b, Dekou et al., 2016]). The contribution of the low-speed regions to the total Reynolds stress was 37\% (on average), while that from the high-speed regions represented $29 \%$. These relative values of Reynolds stress contributions are similar to the values found by [Dekou et al., 2016] of $40 \%$ and $25 \%$ for large-scale low- and high-momentum regions, respectively. The slight oscillations observed in figure 6.28 might be associated to the number of structures used to compute the statistics. These results presented lead to the conclusion that the low- and high-speed regions are associated to the production of turbulent . In addition, by revisiting figure 6.26 , it can be verified a strong relationship between low-speed regions with ejections and between high-speed regions with sweeps in the present data.

Figure 6.28(b) shows the conditional statistics for dissipation rates of lowand high-speed regions together with flow statistics. As already mentioned, the computation of dissipation rate in a tomo-PIV data carries a high level of uncertainty, mainly due to spatial derivatives in a relative coarse grid that filters these quantity and adds noise. Plots of dissipation rate, however, deliver qualitative information about the flow. Dissipation curves from conditional statistics follow the flow statistics in this figure indicating no extra dissipation rate in the low- and high-speed regions. A slight overestimation of dissipation rate for low-speed regions is verified, what can suggest higher noise in the data close to the wall, where the shear was more intense, or the existence of vortices. A similar behaviour of the dissipation curve was also found by [Kang et al., 2007]. In that direct numerical simulation work, the dissipation rate was attributed to the higher probability of the vortex existence inside lowmomentum regions than inside high-momentum regions. These finds were in agreement with [Dennis and Nickels, 2011a] that observed the vast majority of vortices nearby low-speed regions.

Figure 6.29 displays the probability density function of the fluctuating vorticity components $\left(\omega_{x}^{\prime}, \omega_{y}^{\prime}, \omega_{z}^{\prime}\right)$ and its magnitude $\left|\omega^{\prime}\right|$, scaled by inner variables, with respect to the wall-normal position. Except for the first wall-normal position, all fluctuating vorticity components show similar narrow PDF distributions centred on zero. The spreading of the fluctuating vorticity component distributions are linked to the threshold value employed. For smaller threshold values, the distribution tails increased. Figure 6.29(a) shows a non-symmetrical distribution with positive mean value for spanwise fluctuating vorticity of lowspeed regions at $y^{+}=43$. The magnitude of vorticity shows the frequency of occurrence of stronger intensity closer to the wall. In figure 6.29(b), mirrored 
PDF of spanwise fluctuating vorticity is obtained for high-speed regions at around the same position.

The root-mean-square of fluctuating vorticity components employing conditional statistics for low- and high-speed regions are presented in figure 6.30. The root-mean-square of fluctuating vorticity calculated over the entire flow is also plotted for comparison. The results presented indicate that fluctuating vorticity components within both low- and high-speed regions present smaller values than those for the complete flow statistics. The increasing of the absolute value of threshold employed increased the difference between each corresponding structure and flow statistical curve. However, the elevated estimated value of uncertainty hampered the quantification of these results.

From the fluctuating velocity vector averaged within the connected region, the orientation of turbulent structures can be computed by eq. (6.2.16) to (6.2.18). Figure 6.31 shows the probability density function of projection angles onto the streamwise-wall-normal plane $\left(\Phi_{x y}\right)$, streamwise-spanwise plane $\left(\Phi_{x z}\right)$ and wall-normal-spanwise plane $\left(\Phi_{y z}\right)$ of the structure detected in the measured wall-normal-spanwise plane for various distances from the wall. According to figure $6.31(\mathrm{a})$, the high occurrence of $\Phi_{x y}$ and $\Phi_{x z}$ around $-180^{\circ}$ and $180^{\circ}$ indicates that these structures travel aligned to the streamwise direction with lower velocity, as expected. These findings are in agreement with [Lin, 2006], which reported a Laplace distribution for $\Phi_{x z}$. The meandering effect of lowspeed regions reported in the literature ([Hutchins and Marusic, 2007, Lin, 2006, Dennis and Nickels, 2011b]) and observed in the instantaneous fields of figure 6.3(b) is represented by the width of these distributions. The PDF of $\Phi_{y z}$ of low-speed regions starts with a bimodal distribution at low wall-normal positions and tends to a single peak distribution with the increasing distance from the wall. The initial peaks are about $60^{\circ}$ and $-60^{\circ}$, indicating that the main population of low-speed regions are lifted following these directions. High-speed regions show analogous behaviour. In figure 6.31(b), high frequency of $\Phi_{x y}$ and $\Phi_{x z}$ around $0^{\circ}$ indicates that these structures travel aligned to the streamwise direction. The distribution of projection angles onto the wall-normal-spanwise plane displays a symmetrical bimodal distribution with peaks getting closer (higher absolute values) with the increase of wall distance. The initial peaks are about $100^{\circ}$ and $-100^{\circ}$, revealing the main tendency of inward motion of high-speed regions along these directions. Some oscillations suggest that the statistics were not fully converged. 


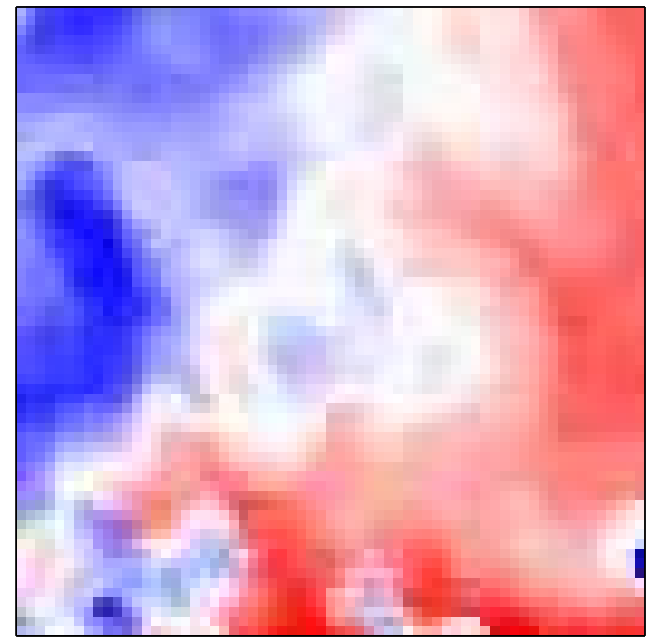

(a)

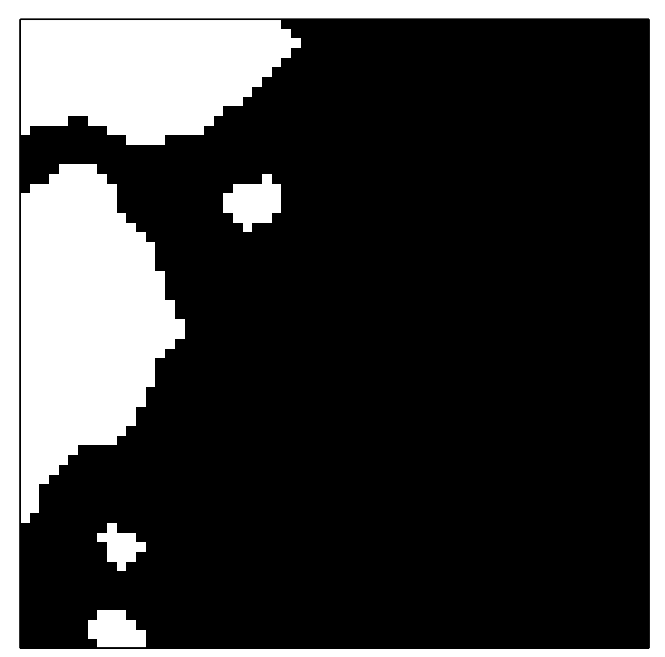

(c)

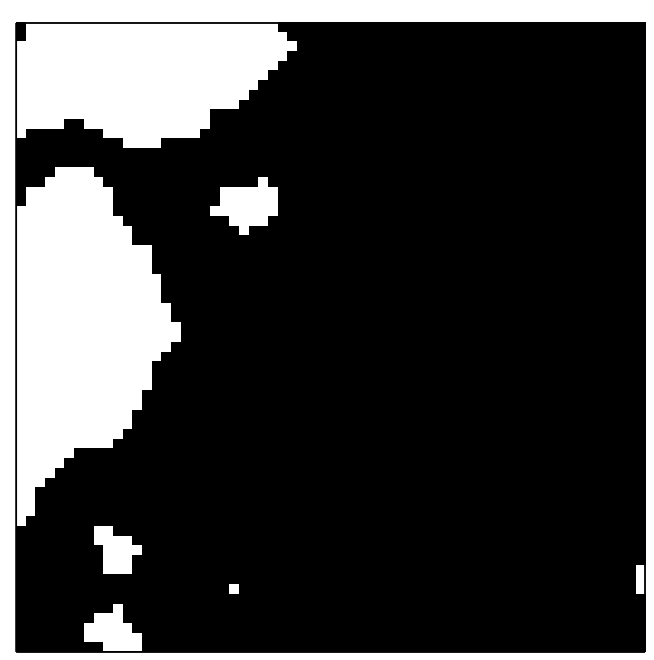

(b)

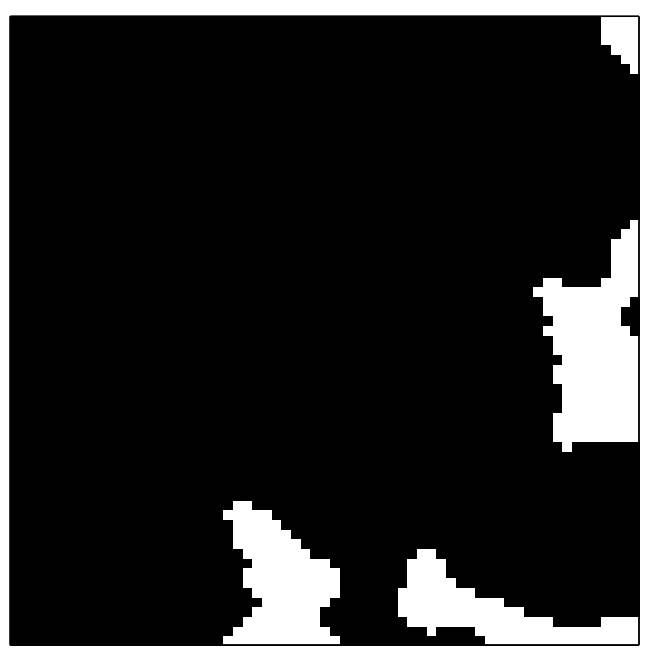

(d)

Figure 6.21: Low- and high-speed region detection procedure in the the middle plane of the tomo-PIV data: (a) colorplot of $u^{\prime} / u_{\mathrm{rms}}^{\prime}$, (b) binary field of lowspeed regions after thresholding, (c) low-speed regions after closing, opening and cleaning operations and (d) binary field of high-speed regions after same binary operations. 


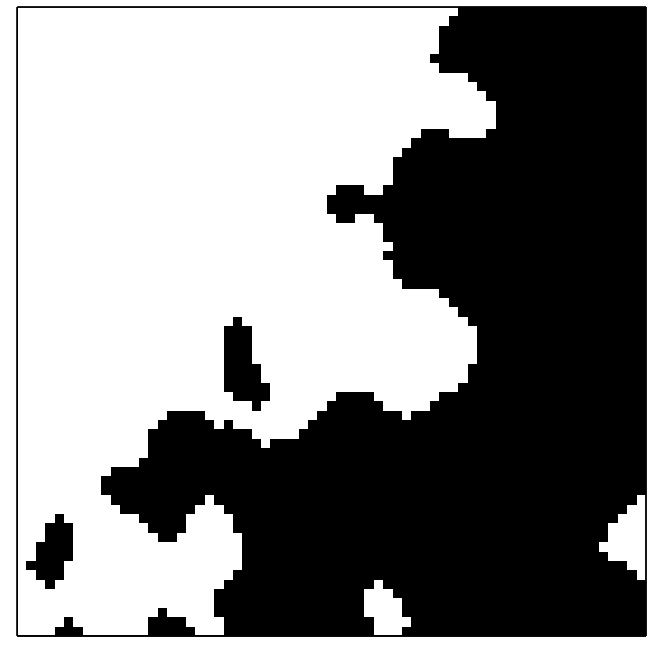

(a)

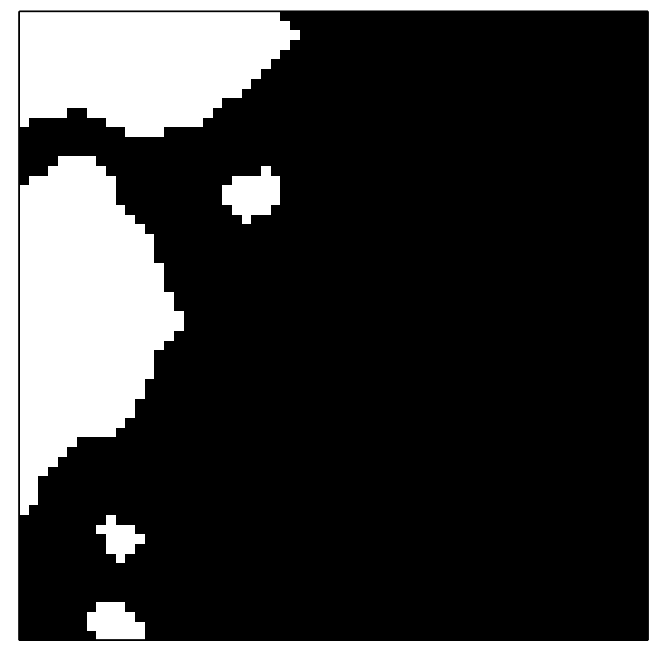

(c)

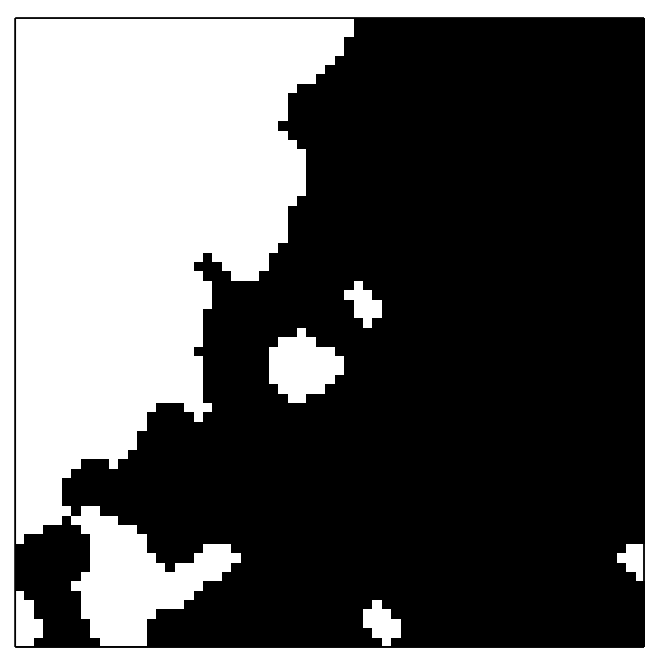

(b)

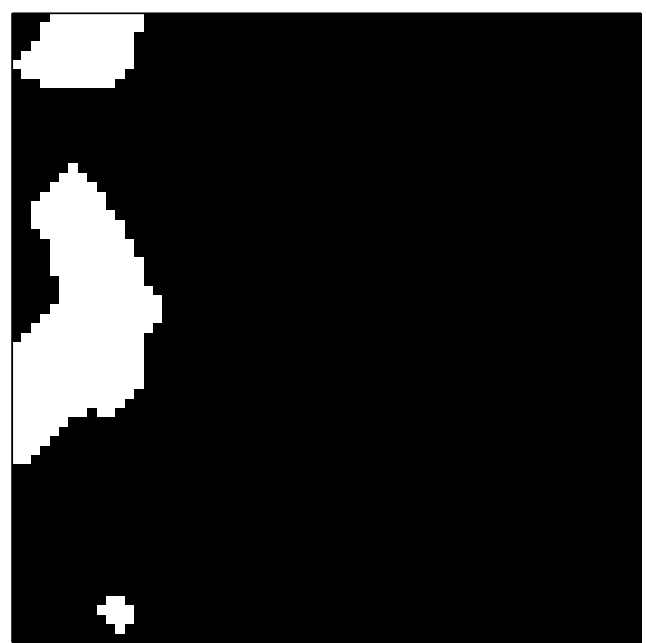

(d)

Figure 6.22: Filtered binary fields of low-speed regions in the the middle plane of the tomo-PIV data for different threshold levels: (a) -1, (b) -1.5, (c) -2 and (d) -2.5 .

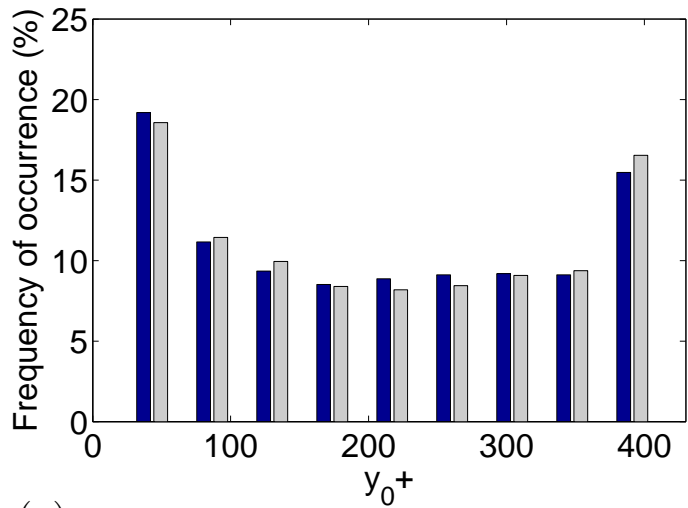

(a)

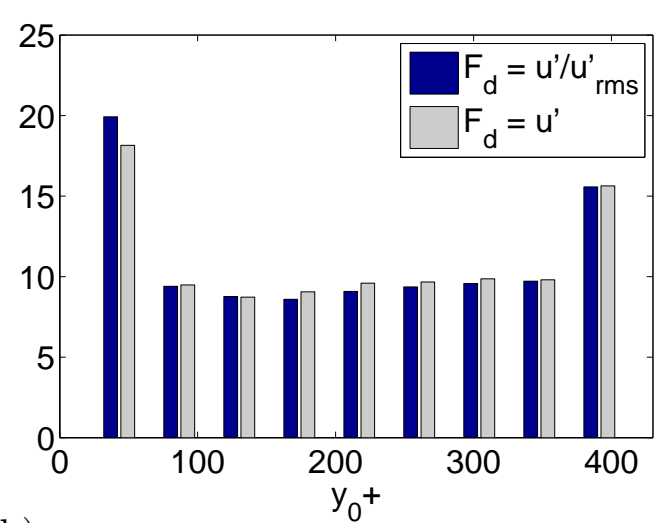

(b)

Figure 6.23: Frequency of occurrence of the centre of turbulent structure detected by standard and normalized fluctuating streamwise velocity for different wall-normal distances. (a) Low- and (b) high-speed regions. 

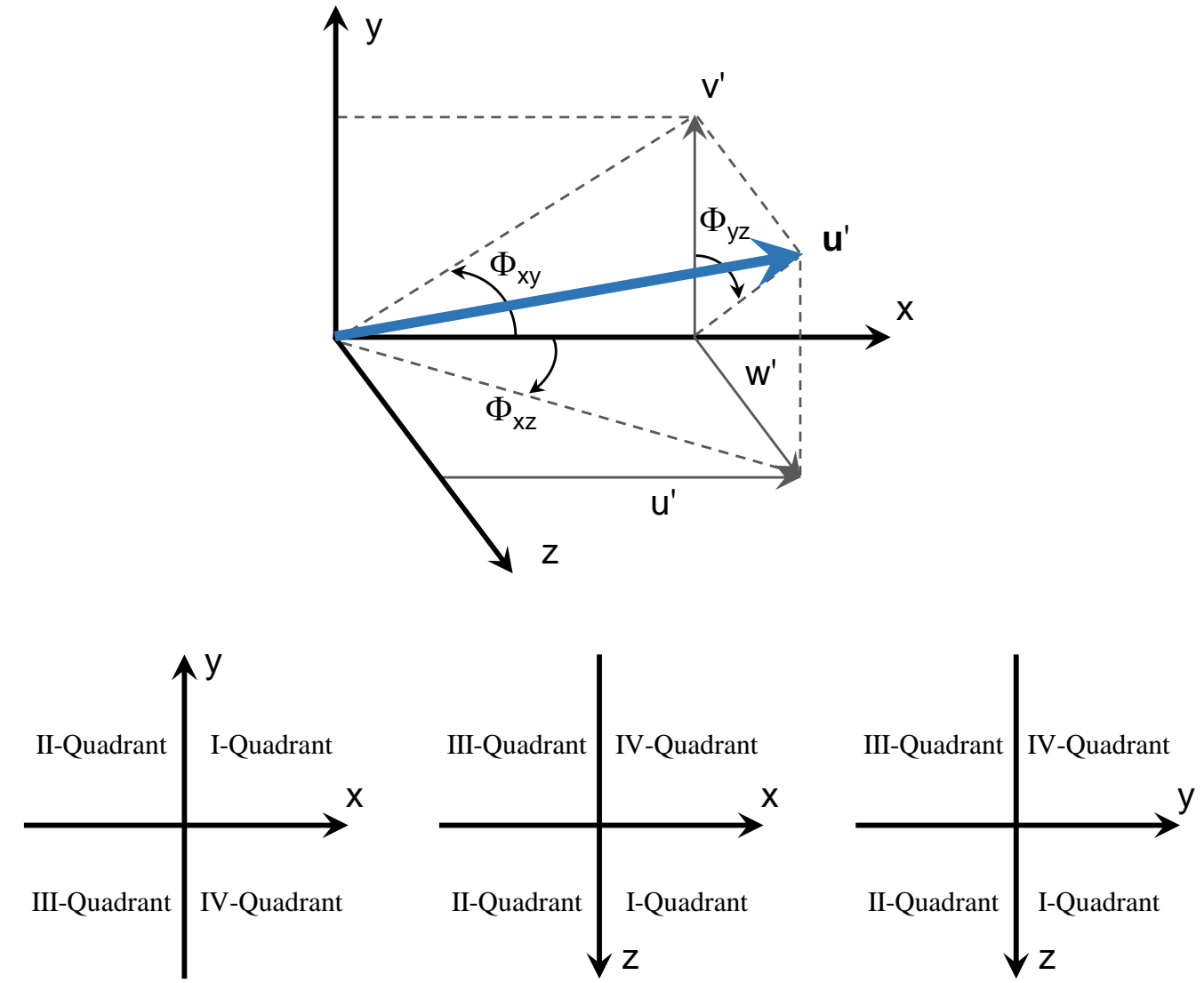

Figure 6.24: Projection angles based on the fluctuating velocity vector and definition of quadrants respectively on streamwise-wall-normal, streamwisespanwise and wall-normal-spanwise planes. 

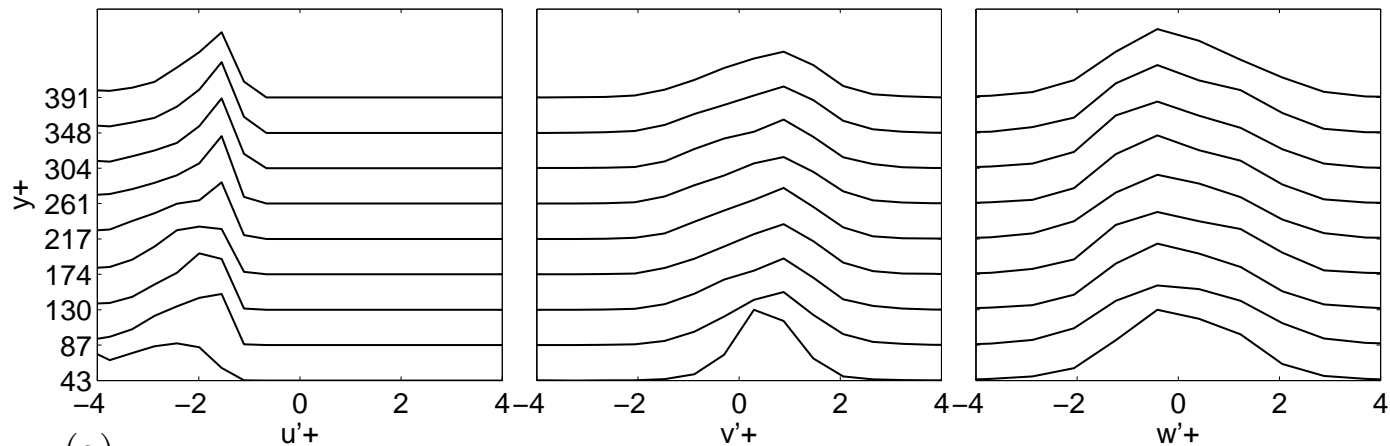

(a)
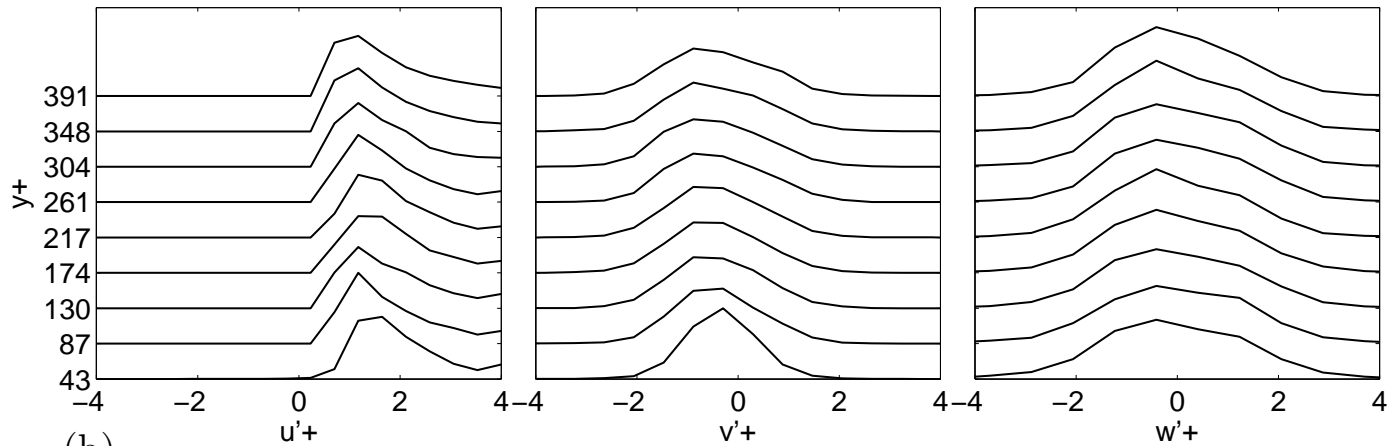

(b)

Figure 6.25: PDF of fluctuating streamwise, wall-normal and spanwise velocities of (a) low- and (b) high-speed regions for different wall-normal positions.
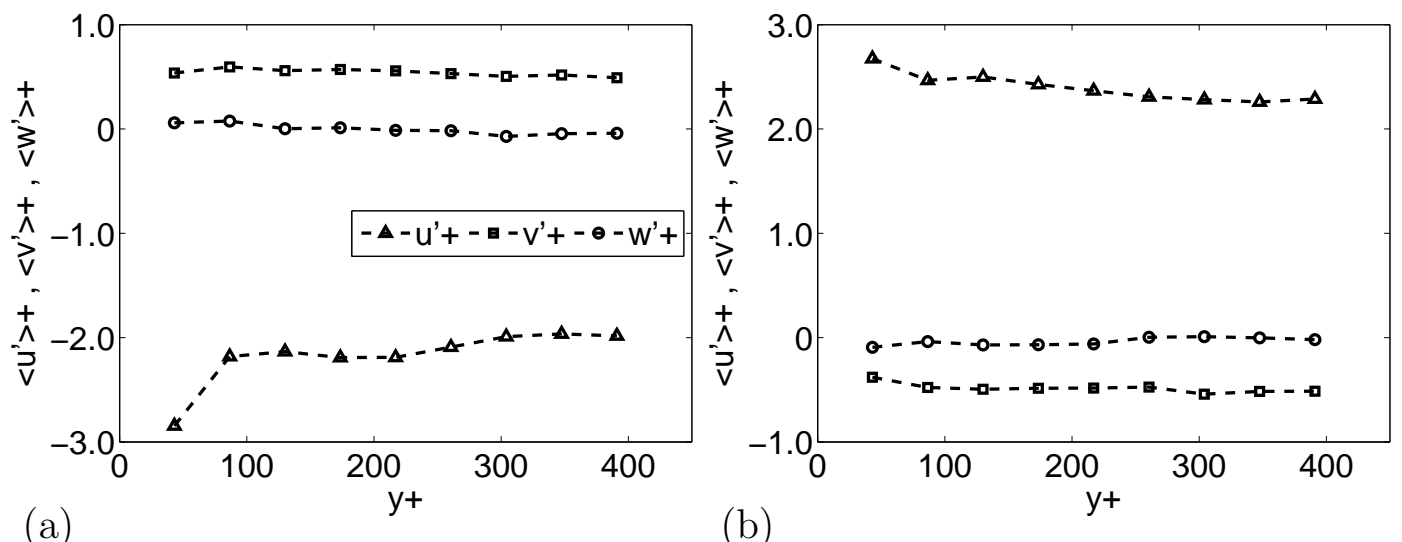

(b)

Figure 6.26: Mean fluctuating velocity components of (a) low- and (b) highspeed regions for different wall-normal positions. 


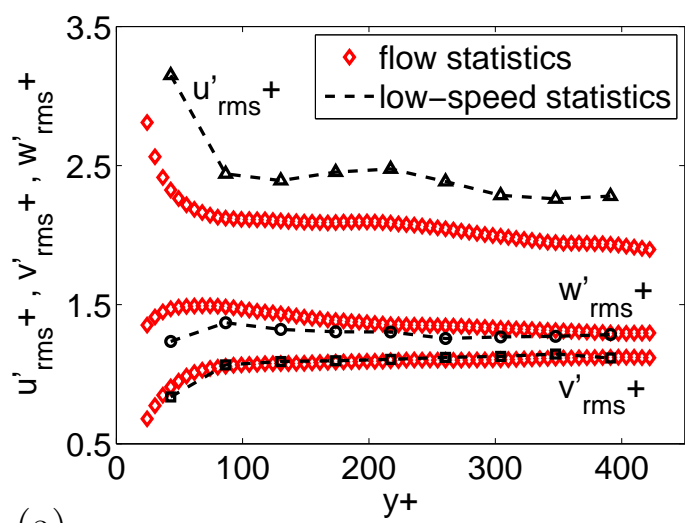

(a)

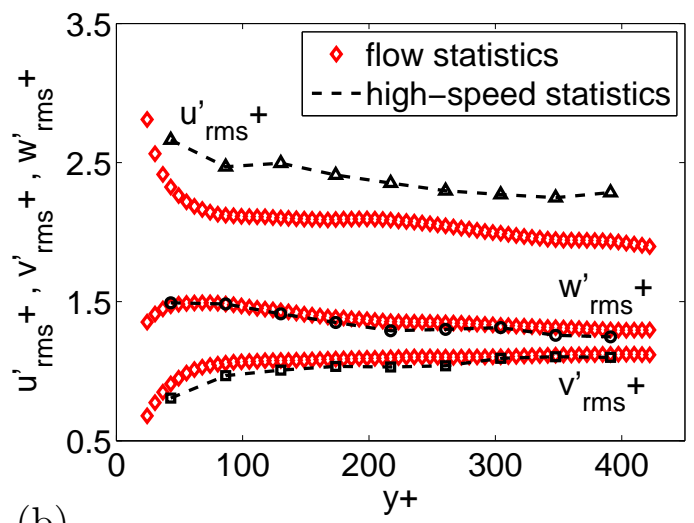

(b)

Figure 6.27: Root-mean-square of fluctuating velocity components of (a) lowand (b) high-speed regions for different wall-normal positions. Flow statistics are also plotted for comparison.

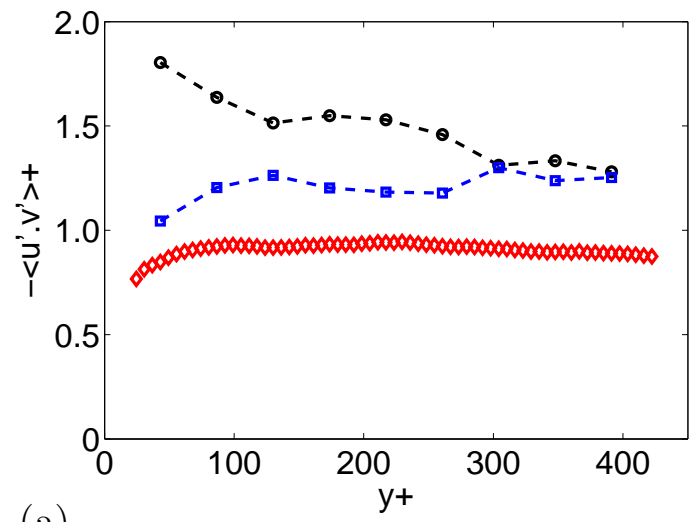

(a)

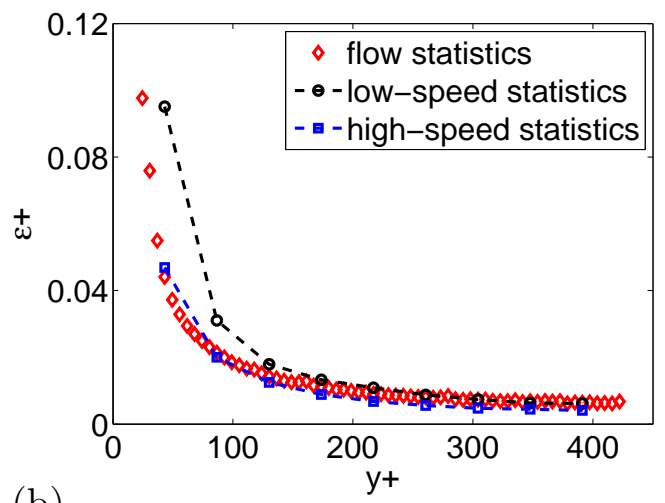

(b)

Figure 6.28: (a) Reynold stresses and (b) dissipation rate of low- and high-speed regions in relation to wall-normal distance. Flow statistics are also presented for comparison. 


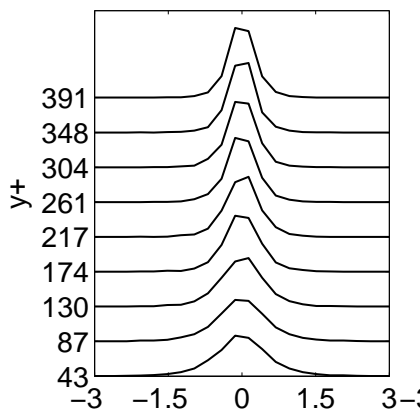

(a) $\omega_{x}^{\prime}+x 10^{-1}$

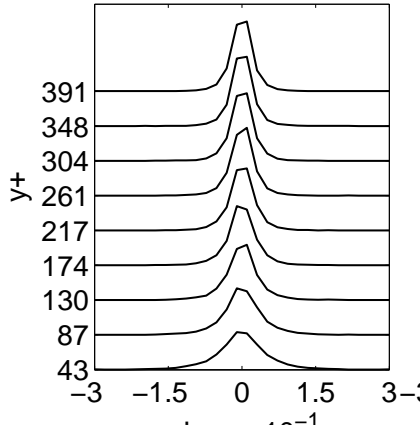

(b) $\omega_{x}^{\prime}+x 10^{-1}$

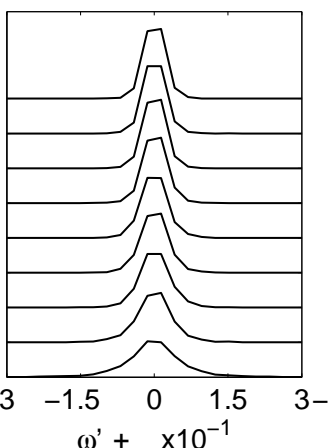

$\omega_{y}^{\prime}+x 10^{-1}$

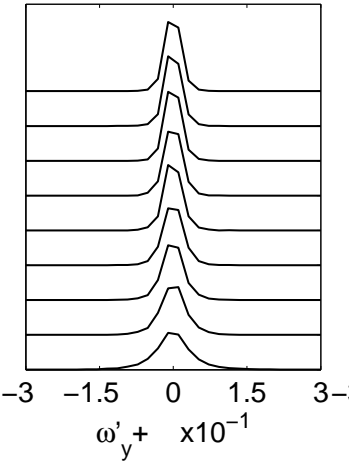

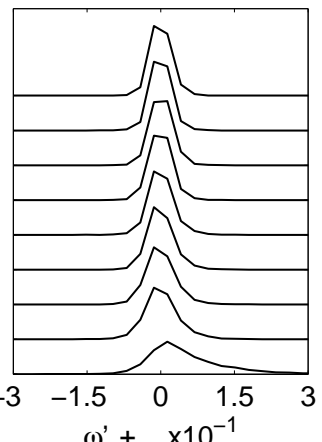

$\omega_{z^{\prime}}^{\prime} \times 10^{-1}$

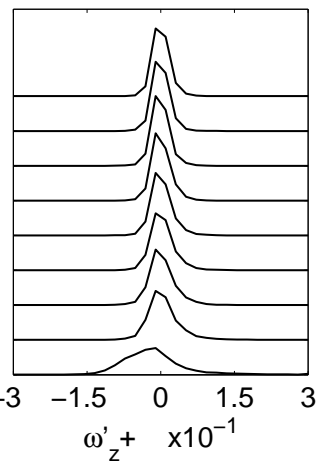

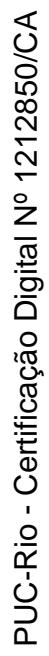

Figure 6.29: PDF of fluctuating streamwise, wall-normal and spanwise vorticities and magnitude of the vorticity vector at (a) low- and (b) high-speed structures for different wall-normal positions.
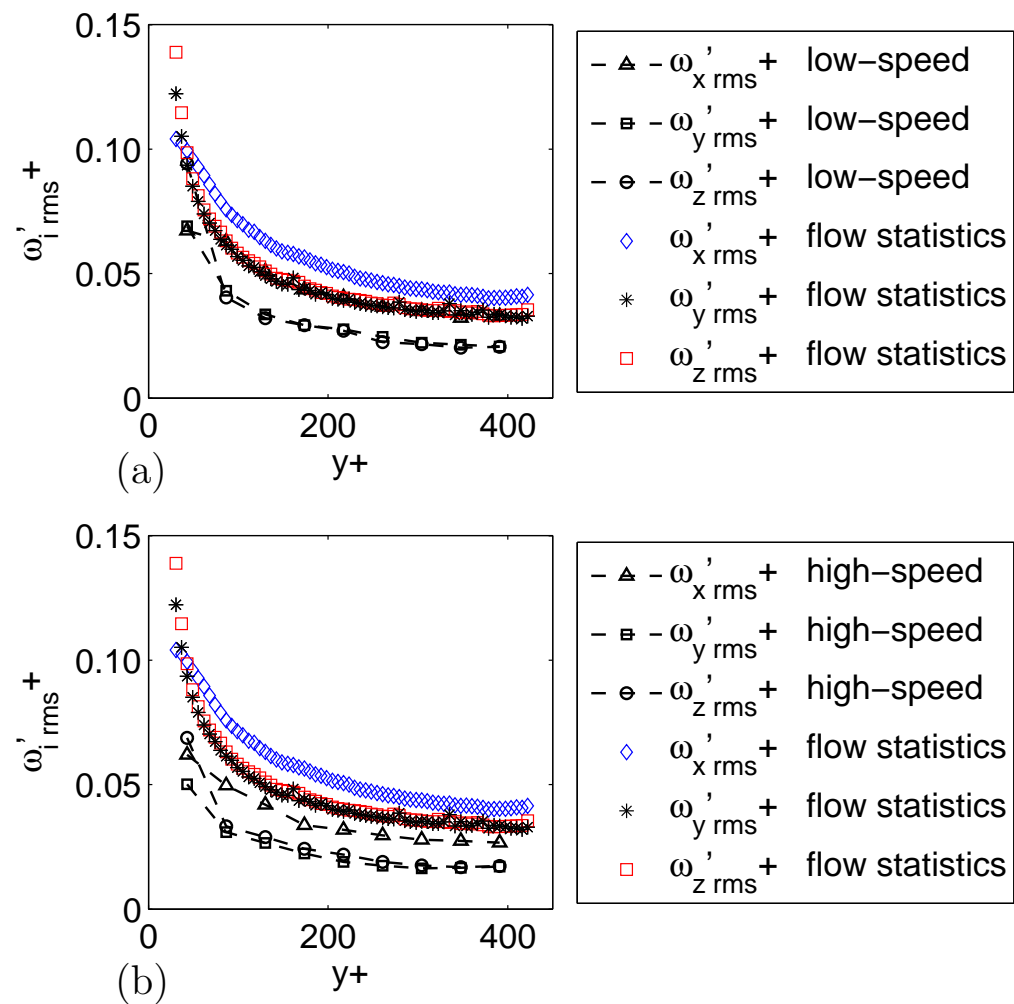

$$
\begin{aligned}
& -\Delta-\omega_{x \mathrm{rms}}^{\prime}+\text { high-speed } \\
& -\omega_{y \text { rms }}^{\prime}+\text { high-speed } \\
& -\theta-\omega_{z \mathrm{rms}}{ }^{\prime}+\text { high-speed } \\
& \diamond \omega_{\mathrm{x} \mathrm{rms}}^{\prime}{ }^{+} \text {flow statistics } \\
& \text { * } \omega_{y \text { rms }}{ }^{+} \text {flow statistics } \\
& \text { - } \omega_{\mathrm{zrms}}{ }^{\prime}{ }^{+} \text {flow statistics }
\end{aligned}
$$

(b)

Figure 6.30: Root-mean-square of streamwise, wall-normal and spanwise vorticities computed in high-speed regions as a function of the distance to the wall. Flow statistics are also plotted for comparison. 

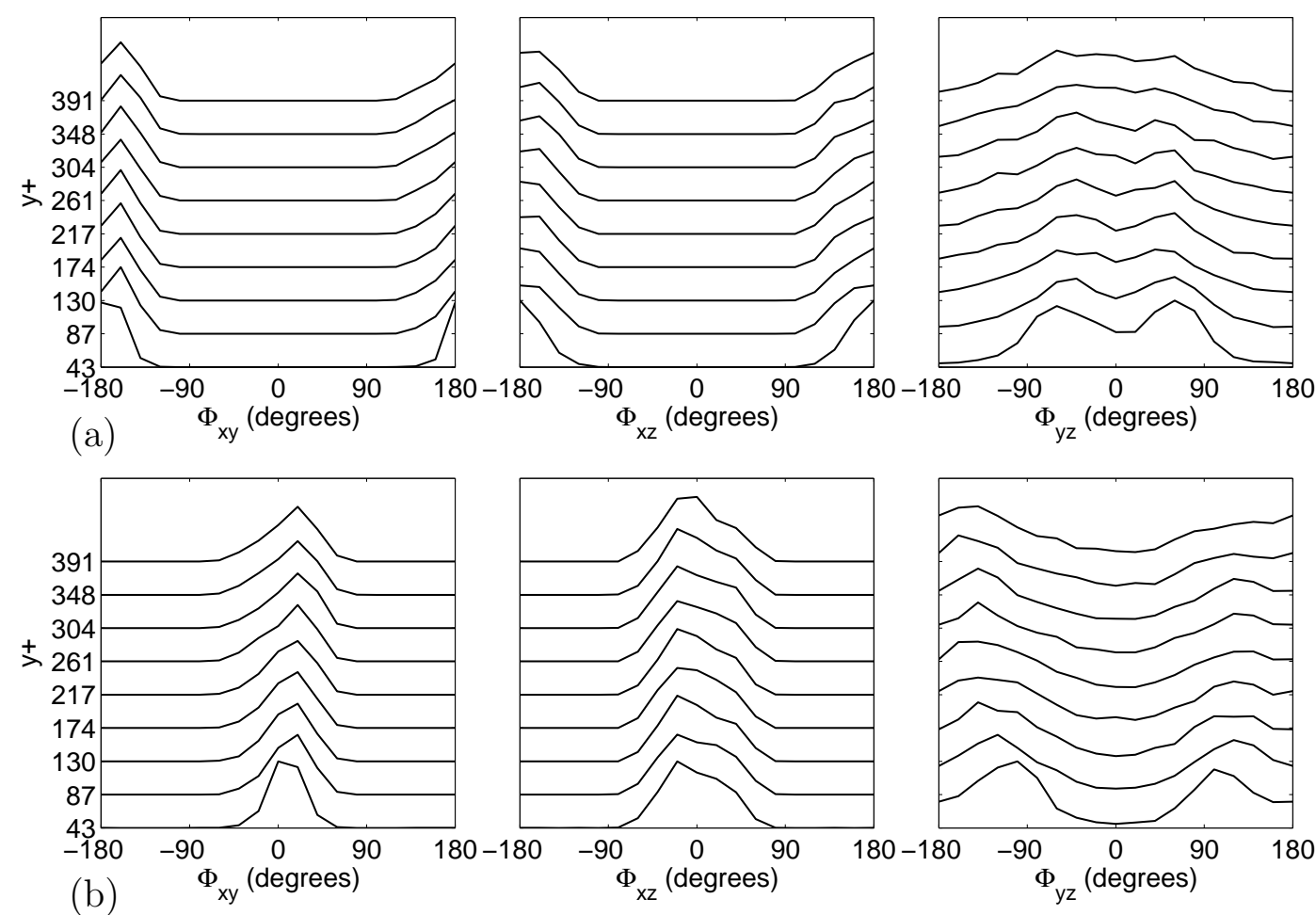

Figure 6.31: Probability density function of projection angles onto the streamwise-wall-normal plane, streamwise-spanwise plane and wall-normalspanwise plane of (a) low- and (b) high-speed regions for different wall-normal positions. 


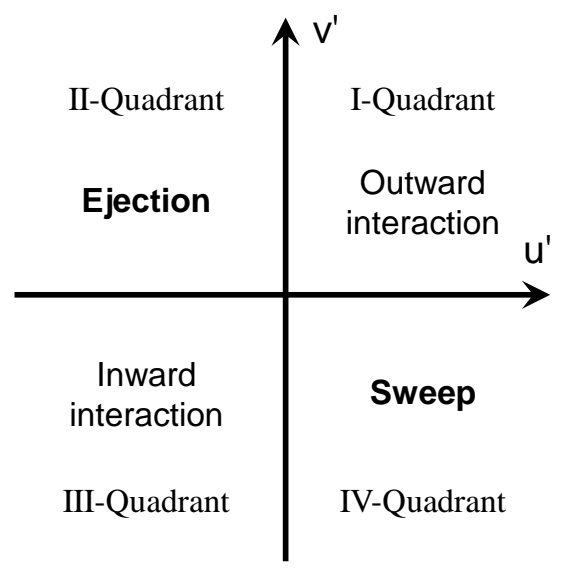

Figure 6.32: Ejection-sweep definitions for momentum transport as proposed by [Wallace et al., 1972]. Events in quadrants 2 and 4 define sweeps and ejections, respectively.

\subsubsection{Ejections and Sweeps}

\section{Ejection and sweep detection and feature extraction}

[Wallace et al., 1972] introduced a quadrant-splitting scheme to detect ejections and sweeps as sketched in figure 6.32. This quadrant algorithm separates the $u^{\prime} v^{\prime}$ signal into four parts. Quadrant two, or just Q2, $\left(u^{\prime}<0\right.$ and $\left.v^{\prime}>0\right)$ is associated with the ejection and quadrant four, or Q4, $\left(u^{\prime}>0\right.$ and $v^{\prime}<0$ ) with the sweep event. The first (associated to outward interaction) and third (related to inward interaction) quadrants contribute negatively to the Reynolds stress in the momentum transport equation, but were found to be scarcer, weaker, and smaller relative to sweeps and ejections. Several others ejection-sweep structure detection algorithms were proposed ([Lin, 2006]), although they were not used in the present work.

In the present study, the ejection and sweep detection function $F_{d}$ was defined as the Reynolds stress normalized by the modulus of its mean $<u^{\prime} v^{\prime}>\left(y^{+}\right)$. Employing this metric, it is possible to generalise the detection function for different Reynolds number and to overcome slightly variations in the $u^{\prime} v^{\prime}$ distribution along the wall-normal direction. This normalization seems to produce better results than the normalization by [Lin, 2006], employing $u_{\mathrm{rms}}^{\prime}\left(y^{+}\right) v_{\mathrm{rms}}^{\prime}\left(y^{+}\right)$. The probability density functions of Reynolds stress and its normalized version for ejection and sweeps are displayed in figure 6.33.

$$
F_{d}^{a}=f\left(u^{\prime}\left(x^{+}, y^{+}, z^{+}\right), v^{\prime}\left(x^{+}, y^{+}, z^{+}\right),<u^{\prime} v^{\prime}>\left(y^{+}\right)\right)=\frac{u^{\prime} v^{\prime}\left(x^{+}, y^{+}, z^{+}\right)}{\left|<u^{\prime} v^{\prime}>\left(y^{+}\right)\right|},
$$

where the streamwise position $x^{+}$refers to the middle plane $\left(x^{+}=0\right)$. An additional detection function was necessary to split ejections and sweeps. 


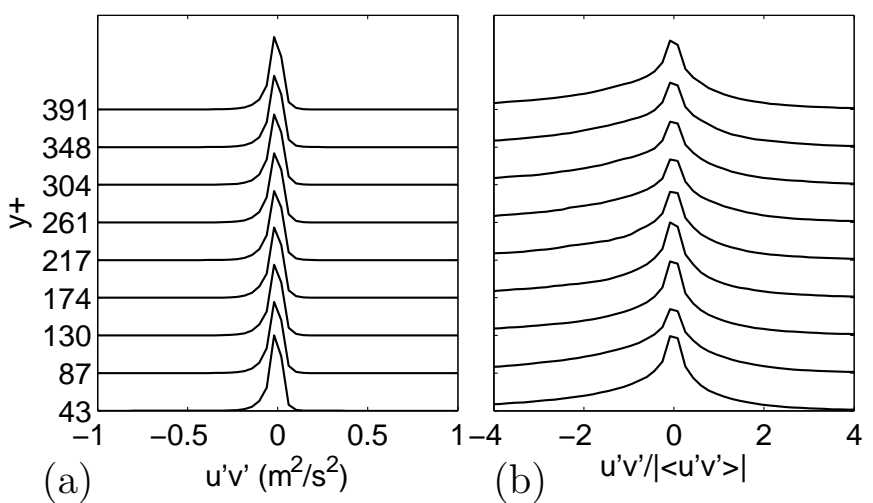

Figure 6.33: PDF of (a) $u^{\prime} v^{\prime}$ and (b) $u^{\prime} v^{\prime} /<u^{\prime} v^{\prime}>$ values used to detect ejection and sweep events for different wall-normal distances.

$$
F_{d}^{b}=f\left(u^{\prime}\left(x^{+}, y^{+}, z^{+}\right), u_{\mathrm{rms}}^{\prime}\left(y^{+}\right)\right)=\frac{u^{\prime}\left(x^{+}, y^{+}, z^{+}\right)}{u_{\mathrm{rms}}^{\prime}\left(y^{+}\right)}
$$

These detection functions represents real scalar fields defined on discrete domains as in the previous section. Ejection and sweep regions were detected applying a negative threshold $F_{\text {thresh }}$ in the $F_{d}^{a}$ field followed by a quadrant filtering employing the $F_{d}^{b}$ field.

$$
\begin{aligned}
& \text { Ejection } F_{B}= \begin{cases}1 & \text { if } F_{d}^{a} \leq F_{\text {thresh }} \text { and } F_{d}^{b}<0 \\
0 & \text { otherwise }\end{cases} \\
& \text { Sweep } F_{B}= \begin{cases}1 & \text { if } F_{d}^{a} \leq F_{\text {thresh }} \text { and } F_{d}^{b}>0 \\
0 & \text { otherwise }\end{cases}
\end{aligned}
$$

The threshold level to classify ejection and sweep regions was selected as -1 , in the present work. This threshold value was chosen after various tests, analogous to the ones described in the preceding section. Similar results about threshold influence were obtained and were in agreement with [Lin, 2006].

Figure 6.34 displays samples of detected ejection and sweep regions by the described method. The $F_{d}$ field of figure $6.34(\mathrm{a})$, being blue mapped to negative and red to positive values, was thresholded producing the binary field $F_{B}$ of figure 6.34(b). This field was filtered by closing and opening operations (employing a structuring element of a $3 \times 3$ diamond shaped) followed by a cleaning procedure to remove noise (erasing structures with areas smaller than about 205 wall units). The filtered binary field of sweep structures is displayed in figure 6.34(c). The binary field of ejection regions in figure 6.34(d) was obtained by similar methodology applied to the same $F_{d}$ field.

Figure 6.35 shows the frequency of occurrence of the distance of ejection and sweep centres $\left(y_{0}\right)$ from the wall. The distance is scaled by inner variables. The turbulent structures were classified using the normalized detection 
function. Each point in the plot represents the statistics over all structures that have their centres within a range around a determined wall distance. This figure shows almost uniform distributions for both detection functions. The watershed algorithm was employed to increase the amount of disconnected regions as explained in section 6.2.2. The total amount of detected ejection regions was about 31000 and sweep regions was about 34000 .

The same features of the low- and high-speed regions were extracted for ejection and sweep regions. For each turbulent structure, fluctuating velocity components, fluctuating vorticity components, Reynolds stress and dissipation rate were averaged within the connected region in the binary fields, and the projection angles of each structure were computed based on the fluctuating velocity components. The conditional statistics were calculated in relation to structures around the same wall-normal position. Geometric characteristics of ejection and sweep structures were not extracted because the small amount of structures (before the application of watershed algorithm) not touching the field borders in the present data.

Ejection and sweep detection and feature extraction were compiled as follows,

1. Detection of all regions with normalized Reynolds stress $\left(u^{\prime} v^{\prime} /\left|<u^{\prime} v^{\prime}\right\rangle\right.$ |) lower than a threshold with the additional condition of fluctuating streamwise velocity lower or higher than zero for ejection or sweep regions, respectively.

2. Closing and opening operations to attenuate noise in the structure geometry.

3. Cleaning operation to filter out small connected regions.

4. Extraction of structure features as the average of these features inside the connected region.

5. Computation of conditional statistics of the various features for different wall-normal positions.

\section{Ejection and sweep statistics}

The displacement of turbulent structures can be inferred from the probability density function of the fluctuating velocity components presented in figure 6.36 with respect to the wall-normal position. The ejection distributions (figure 6.36(a)) are similar to the low-speed distributions (figure 6.25(a)), although higher values of wall-normal velocity are displayed by the former. The 


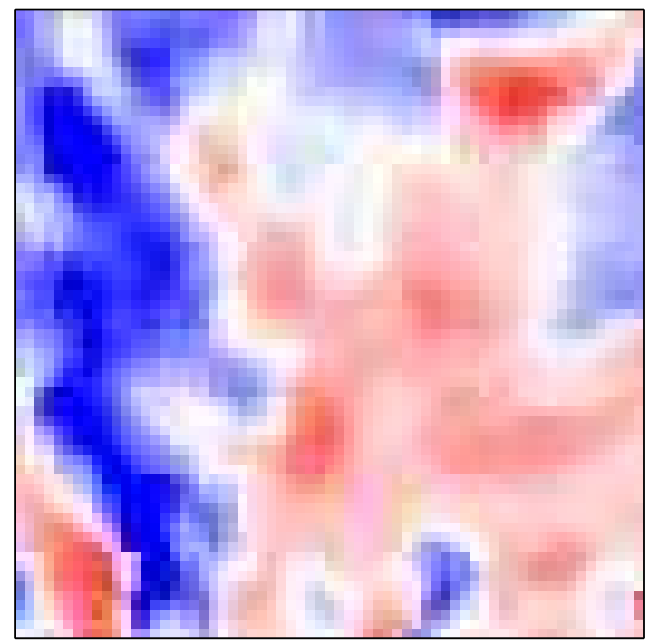

(a)

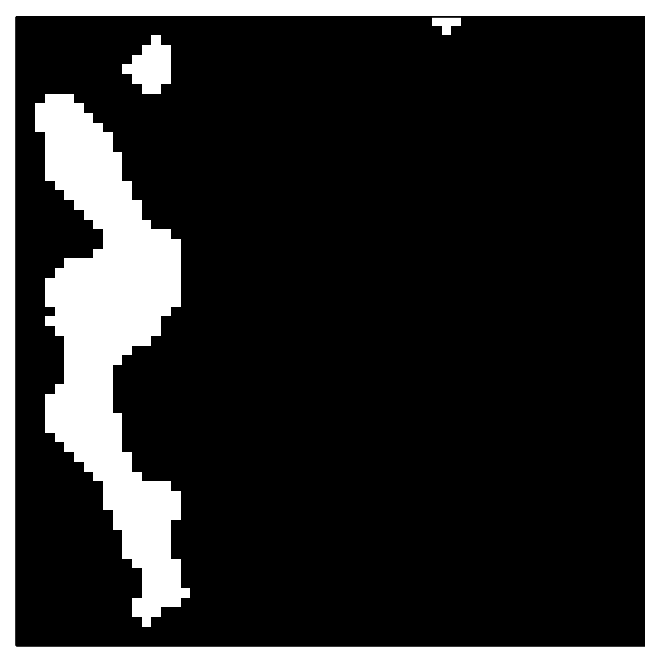

(c)

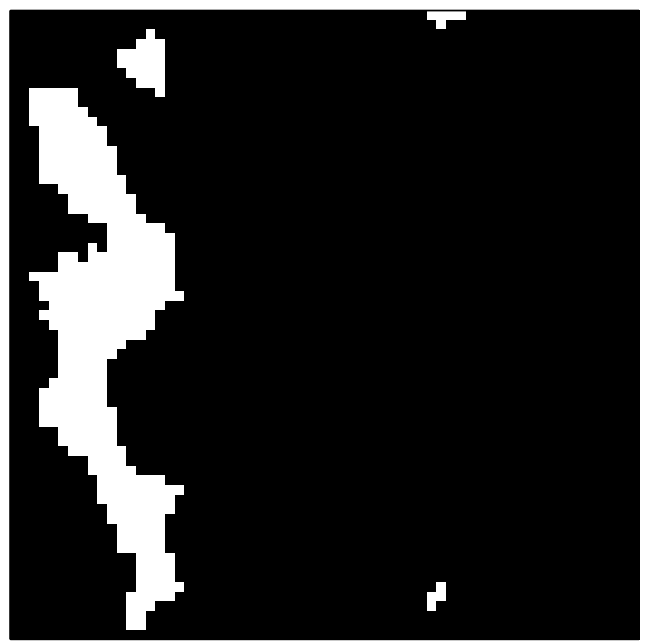

(b)

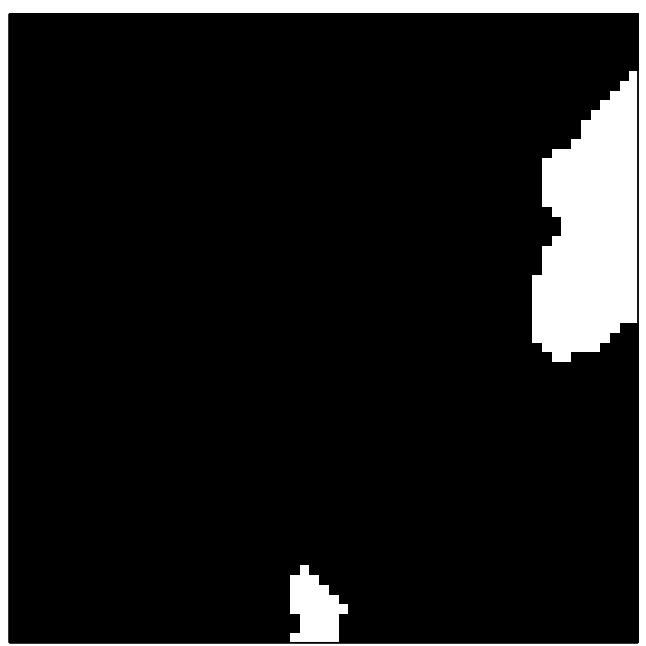

(d)

Figure 6.34: Ejection and sweep detection procedure in the the middle plane of the tomo-PIV data: (a) colorplot of $F_{d}^{a}$, (b) binary field of ejection regions after thresholding, (c) ejection regions after closing, opening and cleaning operations and (d) binary field of sweep regions after same binary operations.

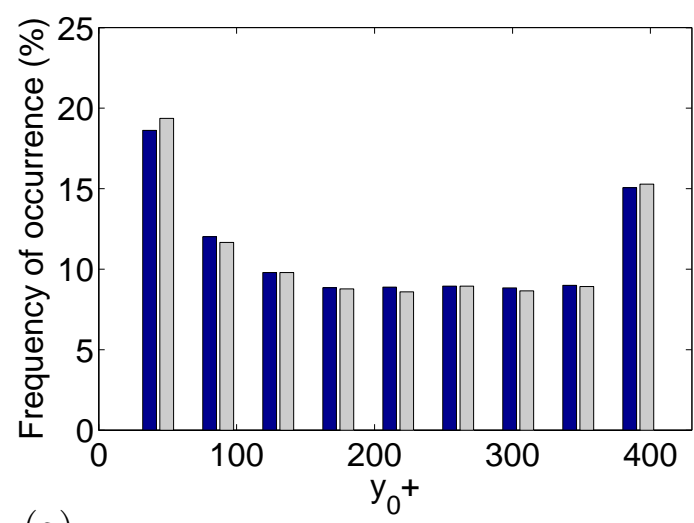

(a)

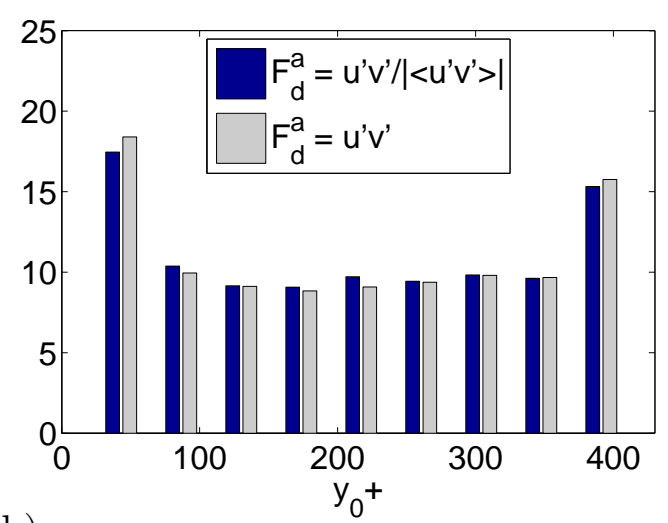

(b)

Figure 6.35: Frequency of occurrence of the centre of turbulent structure detected by standard and normalized Reynolds stress for different wall-normal distances. (a) Ejection and (b) sweep regions. 

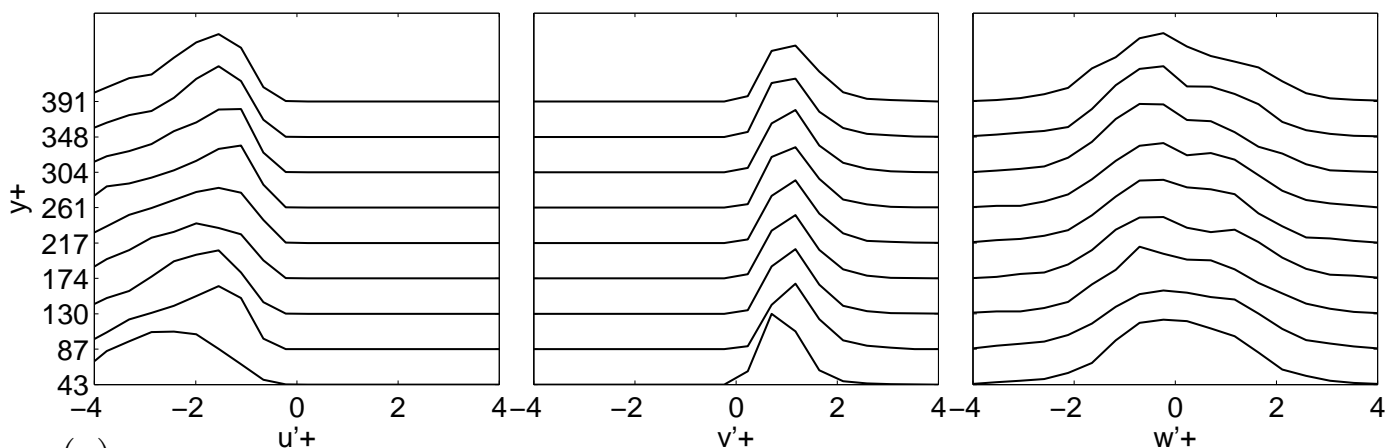

(a)
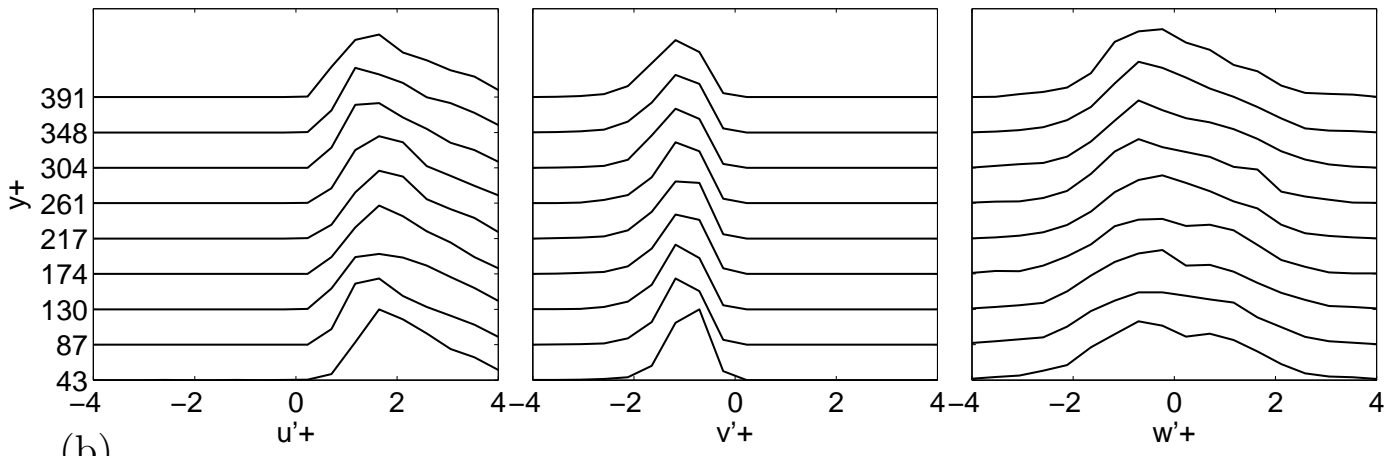

(b)

Figure 6.36: PDF of fluctuating streamwise, wall-normal and spanwise velocities of (a) ejections and (b) sweeps for different wall-normal positions.

sweep PDFs (figure 6.36(b)) also present similar results as the high-speed distributions (figure 6.25(b)) but with lower wall-normal velocities. These results, together with the ones in section 6.2.2, reflect the direct link between lowspeed regions and ejections and high-speed with sweeps and are expected by the quadrant definition of these turbulent structures. In addition, ejection and sweep structures seem to strongly contribute in the average behaviour of lowand high-speed regions. The light oscillations, due to the number of structures considered, did not hamper these analyses.

The same findings can be drawn by the mean fluctuating velocity components within ejection and sweep regions presented in figure 6.37. Both structures display zero mean in the spanwise fluctuating velocities with remarkably high absolute values of the mean of the other fluctuating velocity components. The stream-wise velocity components for ejection and sweeps are higher, in absolute value, than the wall-normal components and present opposite signals. Therefore, ejection regions translate forward lower and upward faster than the neighbouring flow. In addition, these structures move upward, in average, more than two times faster than low-speed regions. An analogous analysis can be made for the sweeps, which flow forward, in average, faster than the surrounding flow and downward faster than high-speed regions.

Figure 6.38 shows a comparison of the root-mean-square of fluctuating 


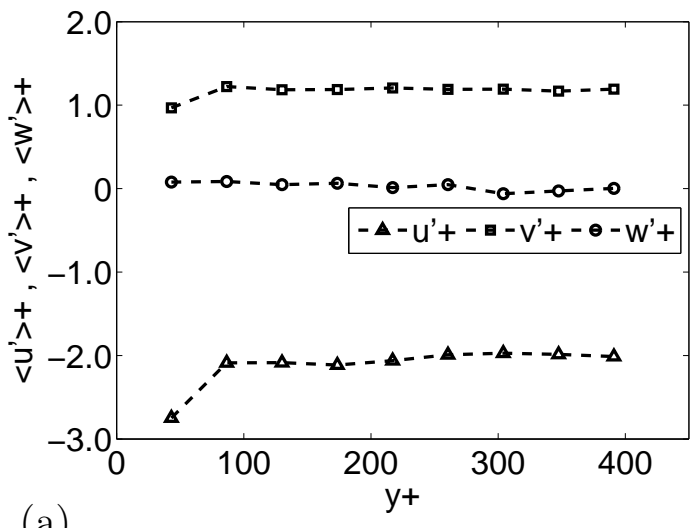

(a)

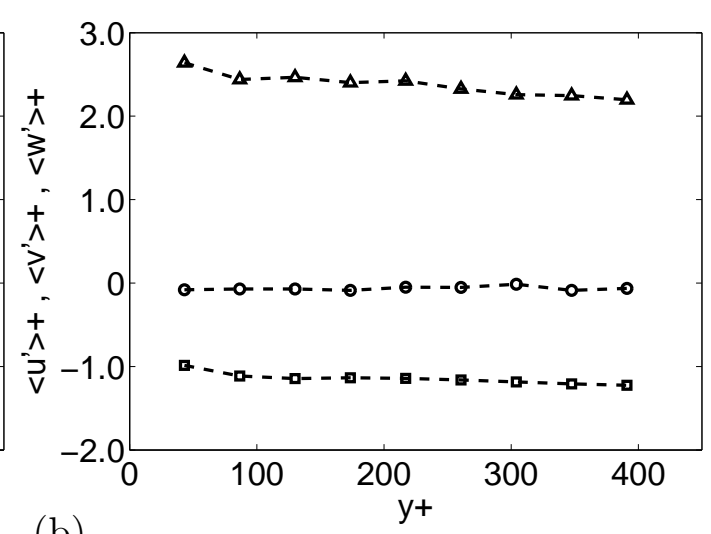

(b)

Figure 6.37: Mean fluctuating velocity components within (a) ejection and (b) sweep regions for different wall-normal positions.

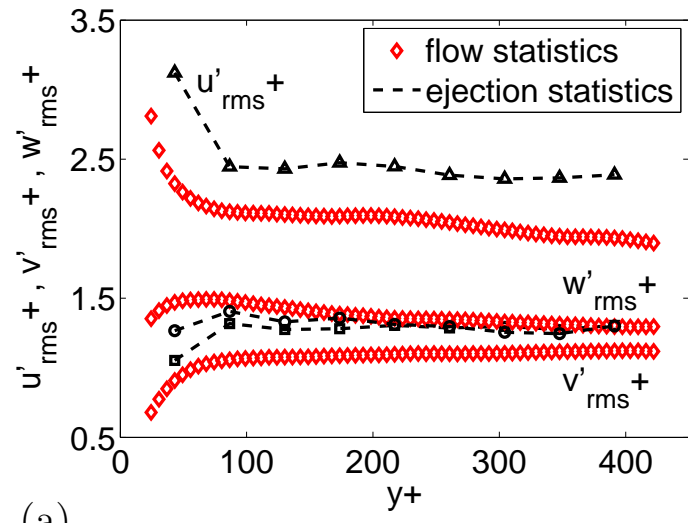

(a)

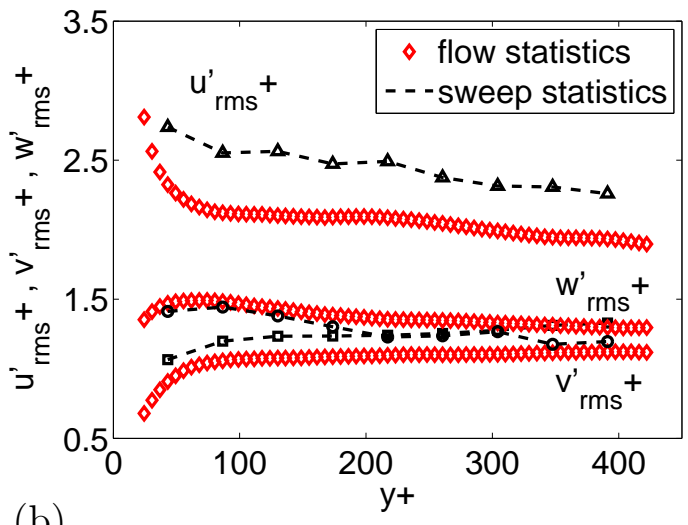

(b)

Figure 6.38: Root-mean-square of fluctuating velocity components of (a) ejection and (b) sweep regions for different wall-normal positions. Flow statistics are also displayed for comparison.

velocity components of ejections, sweeps and those of the entire flow. Profiles of root-mean-square of fluctuating streamwise velocity for ejection and sweep are remarkably above the profiles for the flow statistics. The ejection contribution to the total root-mean-square of fluctuating streamwise velocity was $20 \%$ (on average) with a relative structure area of about $17 \%$ of the total flow area. Sweeps presented the same numbers. It is important to mention that, as expected, large portions of the areas of ejections intercepted the low-speed regions and the same fact occurred relating sweeps and high-speed regions. Regarding wall-normal velocity fluctuations inside ejections and sweeps, they were slightly above the flow statistics. Ejection events contributed with $20 \%$ and sweep events with $19 \%$ to the total root-mean-square of this velocity component. Increasing the absolute value of the threshold maximized the aforementioned differences. The fluctuating span-wise velocity profiles of turbulent structures and the surrounding flow superimpose considering the uncertainty levels of the present tomo-PIV data. 

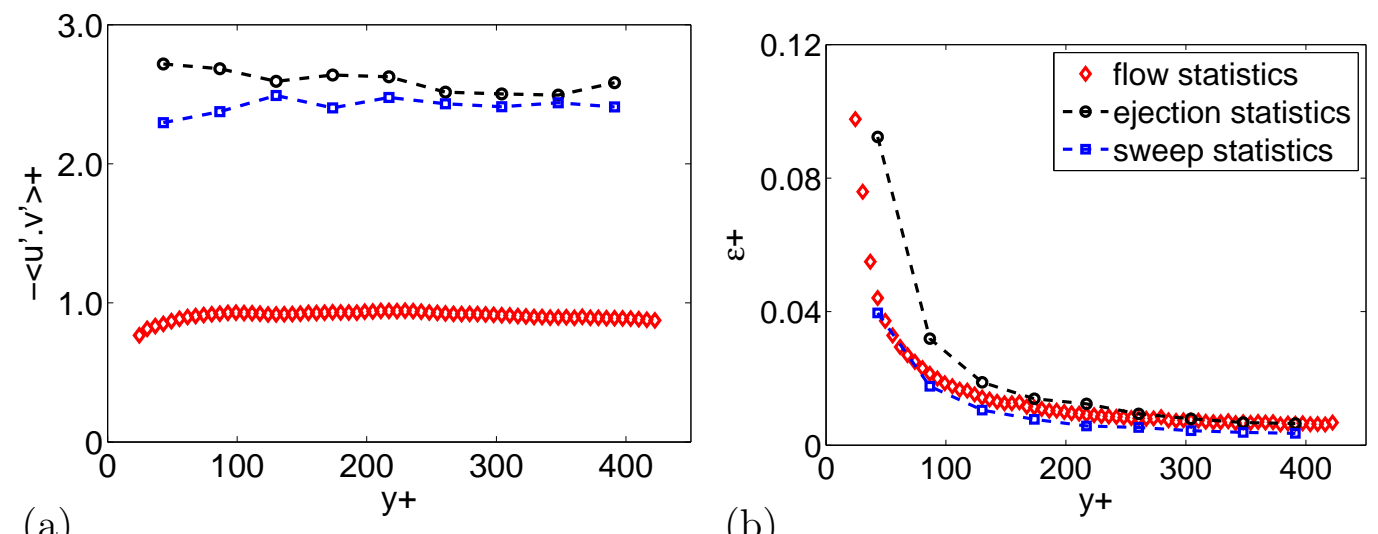

(b)

Figure 6.39: (a) Mean Reynolds stresses and (b) dissipation rate of ejection and sweep regions in relation to wall-normal distance. Flow statistics are also plotted for comparison.

Figure 6.39(a) presents a comparison of mean Reynolds stresses calculated within ejections, sweeps and the flow plotted with respect to wall-normal axis. The conditional statistics display remarkably higher Reynolds stresses than those for the flow statistics and even higher than the mean Reynolds stress for low- and high-speed regions (figure 6.28(a)). The contribution (on average) of the ejection regions to the total Reynolds stresses was higher than that of the sweep regions, being $48 \%$ and $41 \%$, respectively. The observation of higher contribution of ejections in relation to sweeps is in agreement with [Lu and Willmarth, 1973] and [Lin, 2006], although these authors computed even higher values. The discrepancies in the quantification of Reynolds stress contributions of turbulent structures among the works in the literature seems to be influenced by many aspects, namely, the flow geometry, Reynolds number, wall region position, detection techniques or, even, threshold level. The results of figure 6.39 lead to the conclusion that these structures, which are associated to low- and high-speed regions, are the major contributors to the turbulent production, among the turbulent structures studied in this work.

The conditional statistics for dissipation rate within the ejection and sweep regions for different wall-normal positions is displayed in figure 6.39(b). Statistics for the complete flow are also plotted for comparison. Virtually no difference is observed between sweeps and flow curves. Nevertheless, ejections present higher dissipation rates than the surrounding flow close to the wall. This fact seems to be a result of the higher incidence of vortices inside ejection regions. These findings are in agreement with the DNS study of [Kang et al., 2007]. The relative contribution of ejections to the total dissipation rate was, on average, $25 \%$ in the present work.

Figure 6.40 displays the probability density function of the fluctuating 


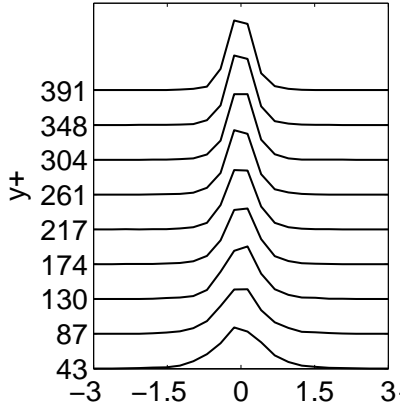

(a) $\omega_{x}^{\prime}+x 10^{-1}$

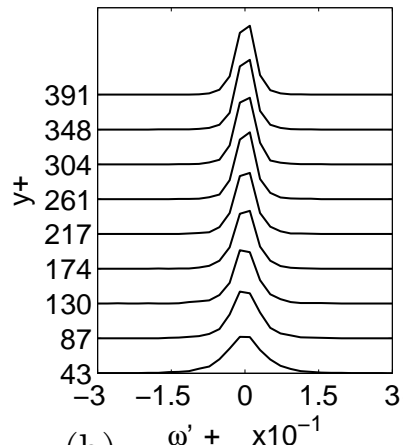

(b)

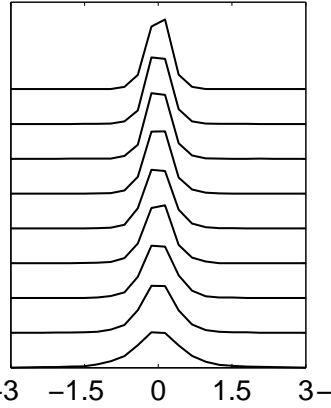

$\omega_{y}^{\prime}+x 10^{-1}$

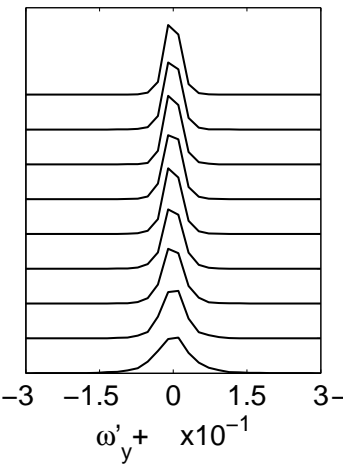

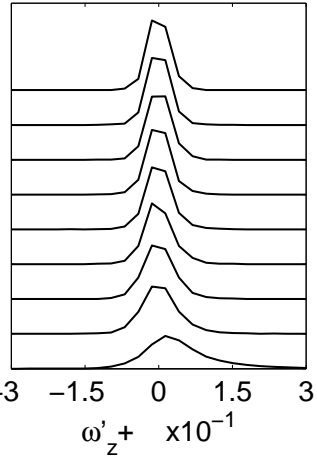

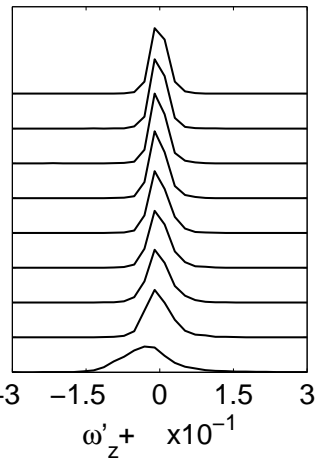

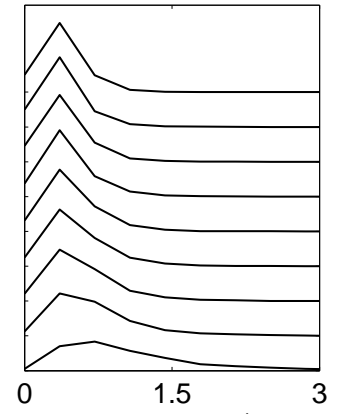

$\left|\omega^{\prime}\right|+\quad \times 10^{-1}$

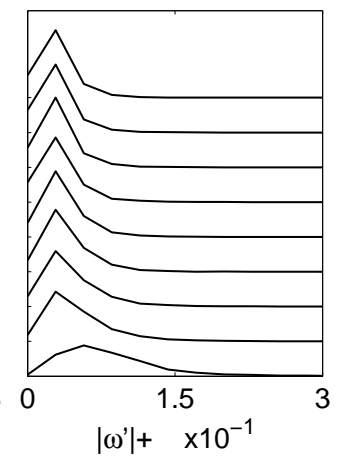

Figure 6.40: PDF of fluctuating streamwise, wall-normal and spanwise vorticities and magnitude of the vorticity vector at (a) ejection and (b) sweep structures for different wall-normal positions.

vorticity components $\left(\omega_{x}^{\prime}, \omega_{y}^{\prime}, \omega_{z}^{\prime}\right)$ and its magnitude $\left(\left|\omega^{\prime}\right|\right)$ inside turbulent structures for different wall-normal positions. Values are scaled by inner variables. Similar results as those of figure 6.29 were obtained, reflecting the correlation between ejection and low-speed regions and sweeps and high-speed regions.

Figure 6.41 presents the root-mean-square of fluctuating vorticity components employing conditional statistics for ejection and sweep regions. The root-mean-square of fluctuating vorticity of the entire flow is also plotted for comparison. The profiles of fluctuating vorticity components of both types of turbulent structures tend to be under those for the entire flow statistics. However, considering the estimated uncertainties $\left(\delta \omega_{i}^{\prime+} \approx 0.04\right.$ in wall variables), ejection statistics are indistinguishable from the flow statistics. These findings indicate higher number of vortex close to ejection regions as compared to sweep regions, complementing the results of the dissipation analyses (figure 6.39(b)). As already mentioned, these conditional statistics values were dependent on the threshold level adopted.

Figure 6.42 shows the probability density function of projection angles onto the streamwise-wall-normal, streamwise-spanwise and wall-normalspanwise planes of ejection and sweep structures for various distances from the 


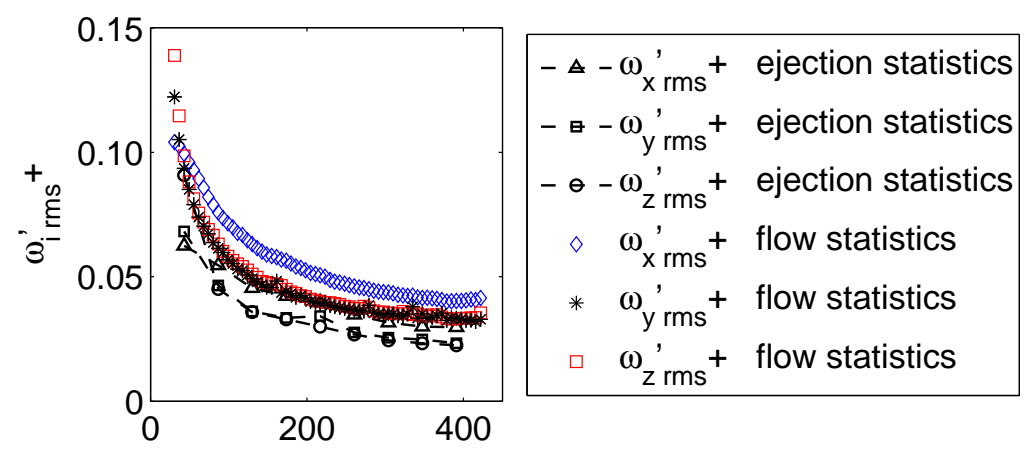

(a) $\quad y+$

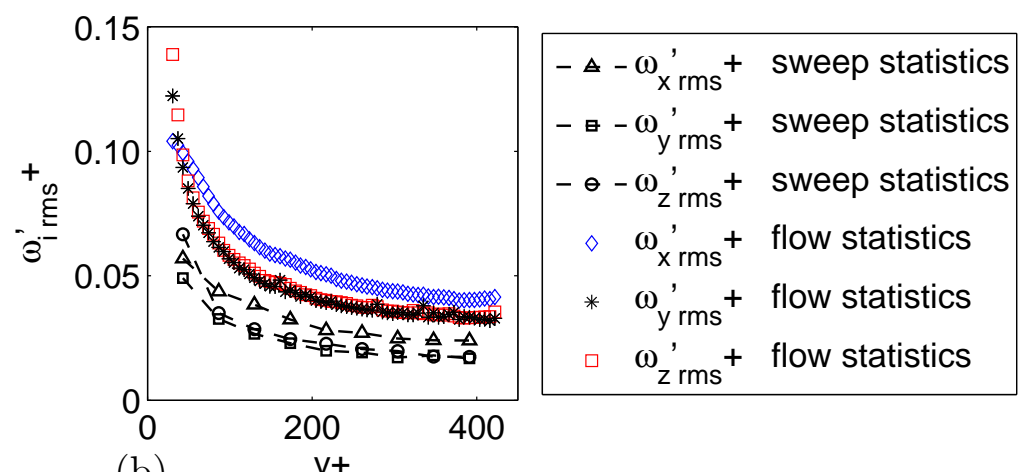

(b)

Figure 6.41: Root-mean-square of streamwise, wall-normal and spanwise vorticities computed in (a) ejection and (b) sweep events in relation to the distance to the wall. Flow statistics are also plotted for comparison.

wall. In figure $6.42(\mathrm{a})$, the high occurrence of $\Phi_{x y}$ slightly lower than $180^{\circ}$, at about $160^{\circ}$, shows the prevailing direction of upward displacement of ejections. Correspondent inward direction of sweep structures, with $\Phi_{x y}$ peak value of about $-10^{\circ}$, is found in figure $6.42(\mathrm{~b})$. These findings are in agreement with [Lin, 2006]. $\Phi_{x z}$ angle expresses the high magnitude of stream-wise velocity component for ejection and sweeps, as already discussed for the corresponding low- and high-speed regions. $\Phi_{y z}$ presents bimodal distributions with similar peak values of figure 6.31 .

\subsubsection{Vortices}

\section{Vortex detection and feature extraction}

Vortices have been investigated in experimental and numerical studies by numerous researchers in an attempt to aid in the understand turbulence. However, no single definition of a vortex is currently a consensus ([Chakraborty et al., 2005, Martins et al., 2016]). Many mathematical definitions to identify vortices have been proposed, such as Q-criterion ([Hunt et al., 1988]), $\lambda_{2}{ }^{-}$ criterion ([Jeong and Hussain, 1995]), $\Delta$-criterion ([Chong et al., 1990]), swirling strength $\left(\lambda_{c i}\right)$ criterion ([Zhou et al., 1999]), fitting to an analytical model 

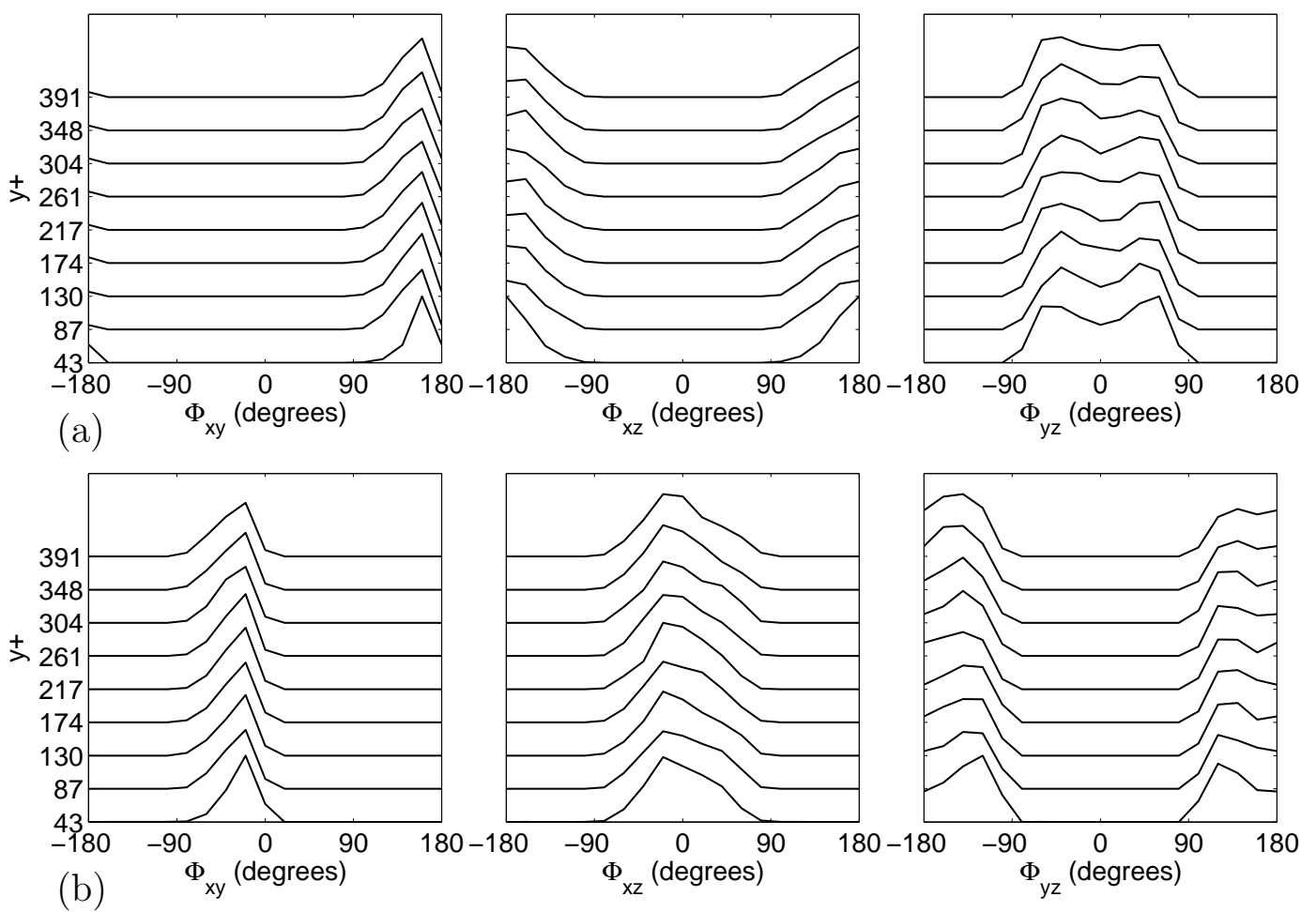

Figure 6.42: Probability density function of projection angles onto the streamwise-wall-normal plane, streamwise-spanwise plane and wall-normalspanwise plane of (a) ejection and (b) sweep regions for different wall-normal positions.

([Vollmers, 2001]), to mention some. [Chakraborty et al., 2005] thoroughly discussed a variety of these detection criteria and concluded that the vortex structures educed by $Q, \lambda_{2}, \Delta$ and $\lambda_{c i}$ were equivalent for the purposes of kinematic and dynamic interpretation.

In the present work, some prior tests were conducted employing the Qcriterion and $\lambda_{2}$-criterion as vortex detection methods. The Q-criterion ([Hunt et al., 1988]) identifies vortices as flow regions with positive second invariant of the velocity gradient tensor, i.e. $Q>0$, with the requirement of pressure in the eddy region to be lower than the ambient pressure. In this work, the additional pressure condition was not used as done by other researchers (e.g., [Jeong and Hussain, 1995, Chakraborty et al., 2005]). The Q-criterion for an incompressible flow is defined as

$$
Q=\frac{1}{2}\left[\|\boldsymbol{\Omega}\|_{F}^{2}-\|\mathbf{S}\|_{F}^{2}\right],
$$

where, from the Frobenius norm and using Euler notation, $\|\mathbf{S}\|_{F}=\left(S_{i j}^{2}\right)^{1 / 2}$, $\|\boldsymbol{\Omega}\|_{F}=\left(\Omega_{i j}^{2}\right)^{1 / 2}, \mathbf{S}=(1 / 2)\left(\partial u_{i} / \partial x_{j}+\partial u_{j} / \partial x_{i}\right)$ is the symmetric component of the velocity gradient tensor and $\boldsymbol{\Omega}=(1 / 2)\left(\partial u_{i} / \partial x_{j}-\partial u_{j} / \partial x_{i}\right)$ is the antisymmetric component of this tensor. The velocity derivatives were calculated 
by a central difference scheme [Lin, 2006].

The Q-criterion equation can be rewritten in a simpler way as

$$
Q=-\frac{1}{2} \frac{\partial u_{i}}{\partial x_{j}} \frac{\partial u_{j}}{\partial x_{i}}
$$

The $\lambda_{2}$-criterion ([Jeong and Hussain, 1995]) is formulated neglecting the unsteady and viscous effects in the symmetric part of the gradient of the incompressible Navier-Stokes equation

$$
\mathbf{S}^{2}+\mathbf{\Omega}^{2}=-\frac{1}{\rho} \frac{\partial^{2}}{\partial x_{j} \partial x_{j}} p
$$

where $p$ is the pressure. The vortex centre is defined as a connected region of local pressure minimum in a plane with two positive eigenvalues of the symmetric tensor $\mathbf{S}^{2}+\Omega^{2}$. The definition is equivalent to the requirement that $\lambda_{2}<0$ at every point inside the vortex core when ordering these eigenvalues as $\lambda_{1} \geq \lambda_{2} \geq \lambda_{3}$. Differently from the Q-criterion, which measures the excess of rotation rate over the strain rate considering all directions, the $\lambda_{2}$-criterion computes this excess only inside a specific plane ([Chakraborty et al., 2005]).

Figure 6.43 displays the comparison among fluctuating vorticity magnitude $\left(\left|\omega_{3 D}^{\prime}\right|\right)$, Q-criterion $\left(Q_{3 D}\right)$ and $\lambda_{2}$-criterion $\left(\lambda_{23 D}\right)$ for vortex identification applied to the middle plane of a $3 \mathrm{D}$-velocity field from the present boundary-layer tomo-PIV data. To facilitate the visualization, only one over two fluctuating velocity vectors are displayed. The magnitude of the fluctuating vorticity vector, computed from the fluctuation velocity field, is not recommended to classify vortices. Since vorticity does not differentiate rotation and shear, figure 6.43(b) shows dark regions that are not only vortex structures. This result was expected, specially in the near wall-region dominated by strong velocity gradients, and is in agreement with the literature ([Robinson, 1991b]). On the other hand, $Q$ (figure 6.43(c)) and $\lambda_{2}$ criteria (figure 6.43(d)) seem to identify correctly vortex centres. These two criteria agrees very well and insignificant differences between these plots seem to be an effect of the colormap used. The equivalence of $Q$ and $\lambda_{2}$ criteria is in accordance with the findings of [Chakraborty et al., 2005]. Note that some noise, expected from experimental data, is also observed in these unfiltered fields.

Although similar results for structure detection were obtained employing Q-criterion and $\lambda_{2}$-criterion, the latter is more time consuming due to the calculation of eigenvalues. In view of that, the Q-criterion was used in the present work. However, the standard Q-criterion could not be employed directly as a detection function, since its intensity distribution varies along the wall-normal axis. This significant variation can be observed in the probability 

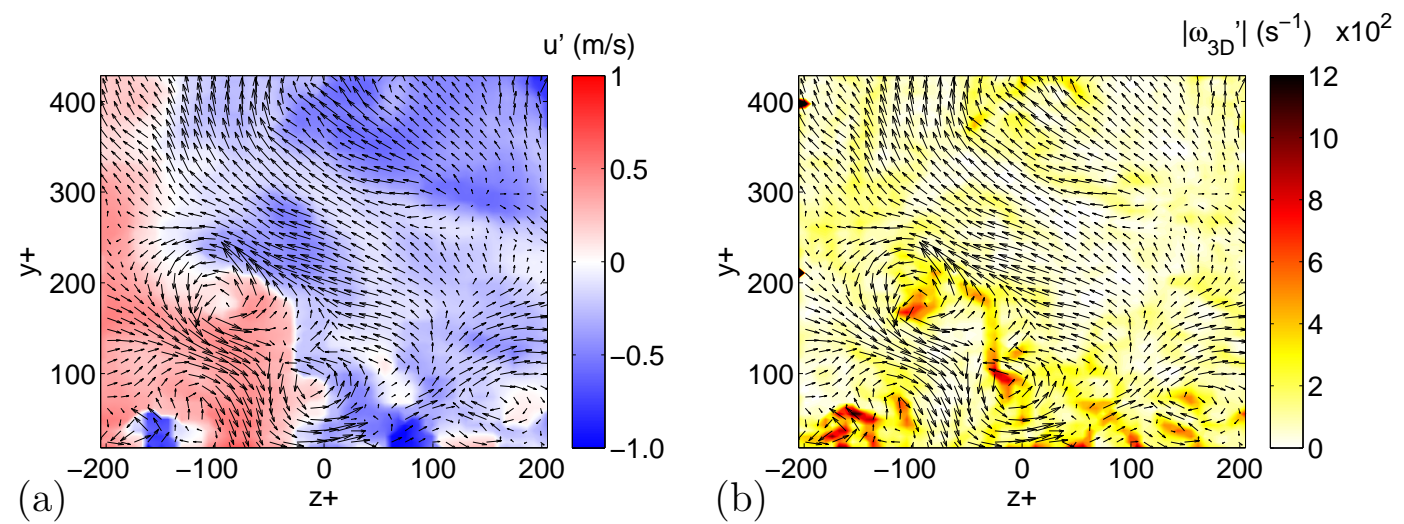

(c)
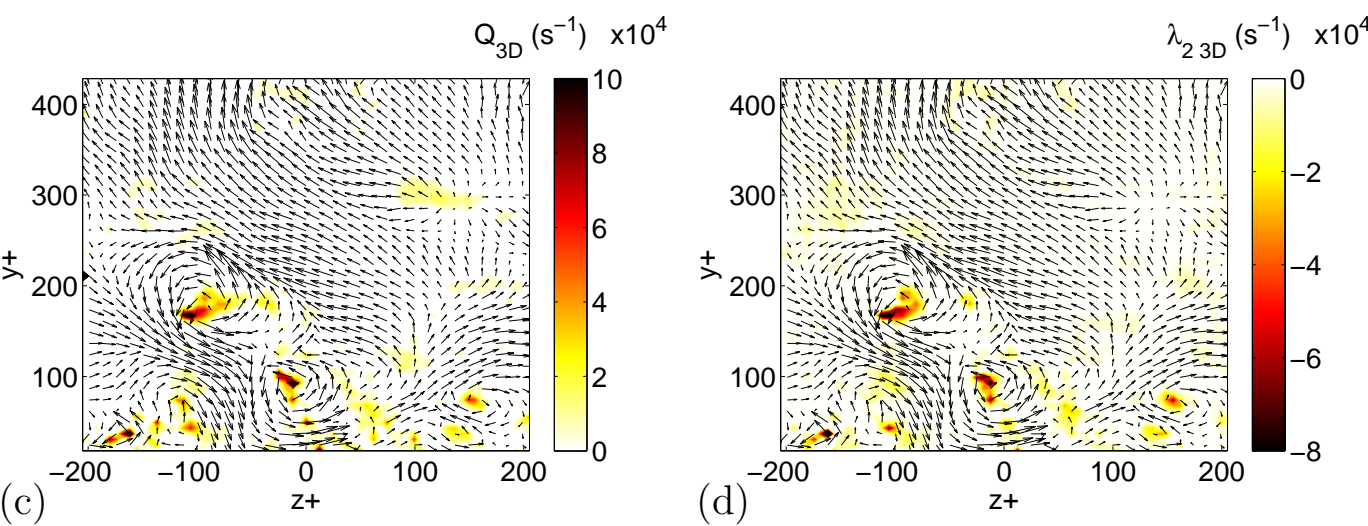

Figure 6.43: Comparison among (a) an instantaneous fluctuating velocity field and its correspondent (b) fluctuating vorticity, (c) Q-criterion and (d) $\lambda$-criterion. Vectors represents the fluctuating velocity field in the the middle plane of the tomo-PIV measurements.

density functions (PDF) of the Q-criterion displayed in figure 6.44(a). On the other hand, from figure 6.44(b), the PDFs of the $Q$ value normalized by its standard deviation $Q_{\mathrm{rms}}\left(y^{+}\right)$change just slightly for $y^{+}>100$.

The changes in the $Q$ value along the wall normal direction has implications on the structure detection. Figure 6.45 displays the binary fields of detected vortices after cleaning operation employing the standard 3D Q-criterion $(Q)$ and its normalised version $\left(Q / Q_{\mathrm{rms}}\left(y^{+}\right)\right)$applied at the same data sample as that of figure 6.47. All vortices close to the wall, i.e. bottom part of the fields, are well detected in both figures 6.45(a) and (b), however the less intense vortices are only detected by the normalized Q-criterion function (figures 6.45(a)). Decreasing the threshold level increases the vortex areas, agglomerating vortices together. The use of normalized detection function produces more uniformly distributed vortices along the wall-normal and spanwise directions.

The statistics of vortex cores presents the same trend mentioned in the previous paragraph. Figure 6.46 displays the frequency of occurrence of the position of vortex centres $\left(y_{0}\right)$ with respect to the wall distance. The position is scaled by inner variables. The vortices were classified using the same detection 

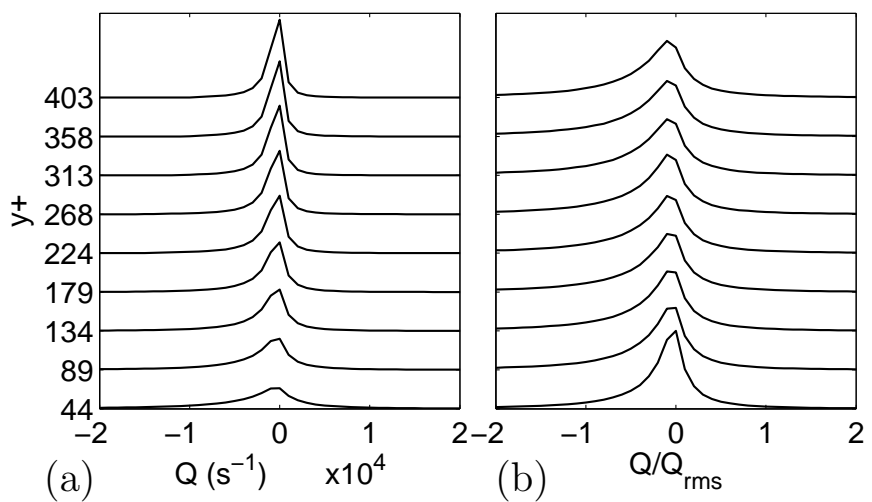

Figure 6.44: PDF of (a) $Q$ and (b) $Q / Q_{\text {rms }}$ values used to detect vortices for different wall-normal distances.

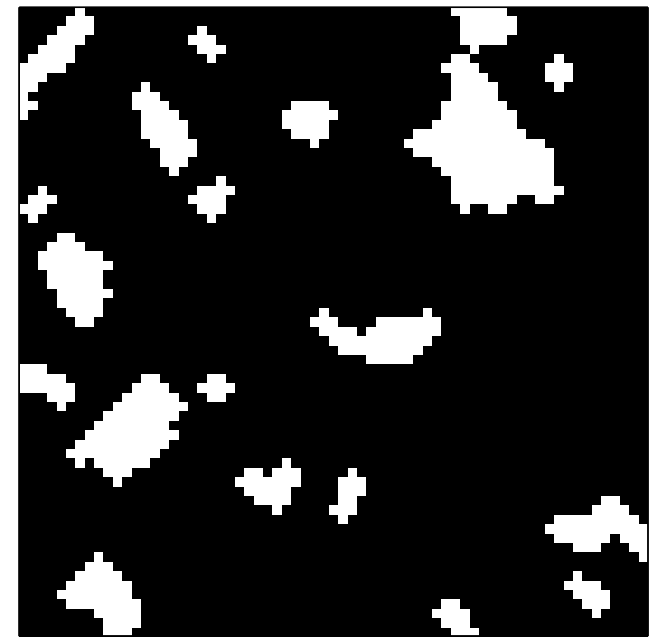

(a)

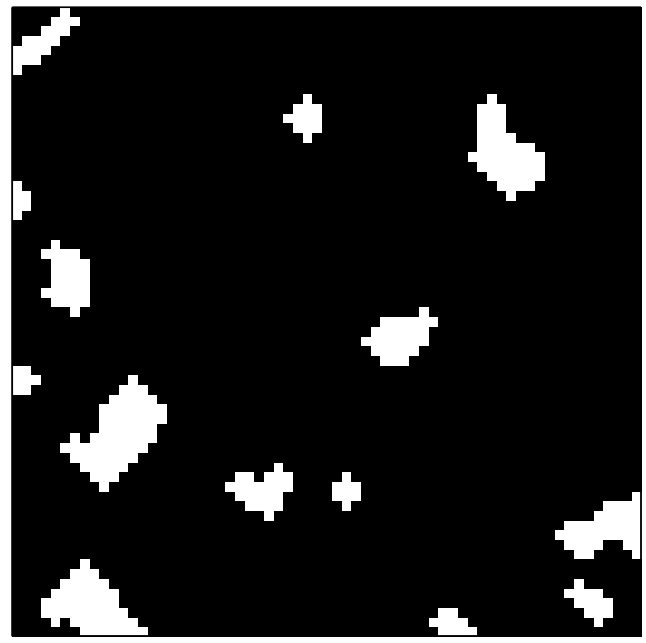

(b)

Figure 6.45: Binary field after cleaning operation of the the middle plane of the tomo-PIV data using different vortex detection functions: (a) $Q / Q_{\mathrm{rms}}\left(y^{+}\right)>$ 0.5 and (b) $Q>2500 \mathrm{~s}^{-1}$.

functions $Q$ and $Q / Q_{\mathrm{rms}}\left(y^{+}\right)$. Each point in the plot represents the statistics over all vortices that have their centres within a range around a determined wall distance. Due to the fact that the function $Q$ varies along the wall-normal direction (figure 6.44) and a fixed threshold was employed, the vortices detected using $Q$ are mainly located near the wall. This is expected since, although the vortices are located all over the field, they are more intense (i.e., higher fluctuating vorticity magnitude and $Q$ values) near the wall. This result is in accordance with the 3D visualizations of other researchers ([Scarano and Poelma, 2009, Atkinson et al., 2011, Schröder et al., 2011, Foucaut et al., 2011a, Jodai et al., 2014]). On the other hand, the use of a normalized $Q / Q_{\mathrm{rms}}\left(y^{+}\right)$shows an almost uniform distribution.

Therefore, in the present study, the vortex detection function $F_{d}^{a}$ is defined as the $Q$ value normalized by its standard deviation. 


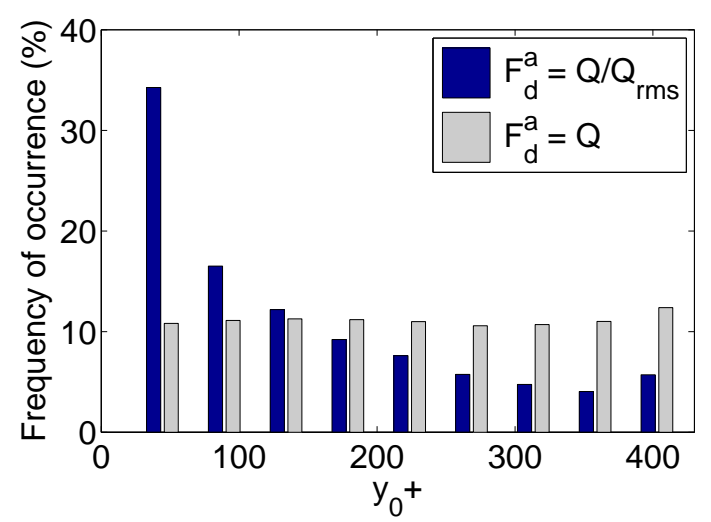

Figure 6.46: Frequency of occurrence of the centre of vortical structure detected by standard and normalized Q-criterion for different wall-normal distances.

$$
F_{d}^{a}=f\left(Q\left(x^{+}, y^{+}, z^{+}\right), Q_{\mathrm{rms}}\left(y^{+}\right)\right)=\frac{Q\left(x^{+}, y^{+}, z^{+}\right)}{Q_{\mathrm{rms}}\left(y^{+}\right)}
$$

where the streamwise position $x^{+}$refers to the middle plane $\left(x^{+}=0\right)$.

The above detection function, applied to the tomo-PIV data, was a real scalar field defined on a discrete domain, as the previous turbulent structures studied. Vortex are detected applying a threshold $F_{\text {thresh }}$ in the $F_{d}^{a}$ field.

$$
\text { Vortices } F_{B}= \begin{cases}1 & \text { if } F_{d}^{a} \geq F_{\text {thresh }} \\ 0 & \text { otherwise }\end{cases}
$$

In the present study, the threshold lower level was selected as 0.5 to classify vortices. This threshold value was chosen after various tests and it was based on the balance between the amount of detected vortical structures and the spurious regions. Lower threshold values created binary fields with fewer amount of large structures, while higher values decreased significantly the size and amount of detected structures. Around the selected value of threshold, the structure shape slightly modify. This behaviour of marginal changes after the optimization of the threshold value is in agreement with other researchers (e.g., [Ganapathisubramani et al., 2006, Lin, 2006]).

The noise in the structure geometry was filtered by one iteration of the closing operation and other of opening operation, employing a structuring element of a $3 \times 3$ diamond shaped. In the present study, small remaining vortices were removed by applying the cleaning operation with an area threshold of 205 wall units, which is equivalent to 5 grids. This area corresponds to vortices with a radius of about 8 wall units, or lower than $2.5 \eta_{k}$ (Kolmogorov length scale), considering $\eta_{k} \approx 3$ wall units ([Carlier and Stanislas, 2005]). Given that the radii of the most intense vortical structures are reported to be around 3 to $5 \eta_{k}$ ([Jiménez et al., 1993, Stanislas et al., 2008, Herpin et al., 2013]), 
only noise and not relevant small vortices were filtered out by the cleaning operation.

Figure 6.47 exemplifies the vortex detection procedure. The detection function field, displayed in figure 6.47(a), is thresholded by a constant value producing the binary field in figure 6.47(b). The colorplot displays only positive values of the detection function field $Q / Q_{\mathrm{rms}}$ with colorbar code equivalent to the one in figure 6.43(c) being white mapped to zero and dark orange to 6 . Note that noise in the $Q / Q_{\mathrm{rms}}$ field appears as irregularities on the object surfaces, disconnected regions, holes and tiny objects in the binary field. Most of these problems are corrected after the mathematical morphology operations of close and open, as can be seen in figure 6.47(c). Small remaining objects (connected white color) are filtered out by a cleaning procedure resulting in the field displayed in figure $6.47(\mathrm{~d})$.

Since the vortex region detection is based on the threshold value, it is expected that some close vortices can be wrongly identified as belonging to a larger object. To solve this possible problem, the watershed operation, which was explained in the section 6.2.1, was tested. This mathematical morphology operation disconnects regions based on the simulated filling of local minima (analogously called as catchment basins). In the present work, the $2 \mathrm{D}$ real matrix $D$ in eq. (6.2.7) refers to the negative of the detection function $F_{d}$ masked with the binary field $F_{B}$. Note that, in the present case, $D$ is composed only by negative real values given by

$$
D=\left\{\begin{array}{cl}
-F_{d} & \text { if } F_{B}=1 \\
0 & \text { otherwise }
\end{array} .\right.
$$

Figure 6.48(a) shows the watershed operation applied just after the closing and opening operations. Small objects remained after the watershed partitioning are removed by cleaning operation resulting in figure 6.48(b). The same middle plane of tomo-PIV data of figure 6.47 is used to facilitate the understanding of the process.

The watershed operations seems to produce similar results as the growing algorithm from local peaks proposed by [Ganapathisubramani et al., 2006] and later used by [Gao et al., 2011]. Unfortunately, probably due to the noise in the $Q / Q_{\mathrm{rms}}$ field (please revisit figure 6.47(a)), the watershed oversegmented the binary field. Regions that appears to belong to the same vortex structure are partitioned into an island composed by smaller structures. This oversegmentation problem is reported in the literature ([Roerdink and Meijster, 2000, Rettmann et al., 2000, Belaid and Mourou, 2011]). This non physical be- 


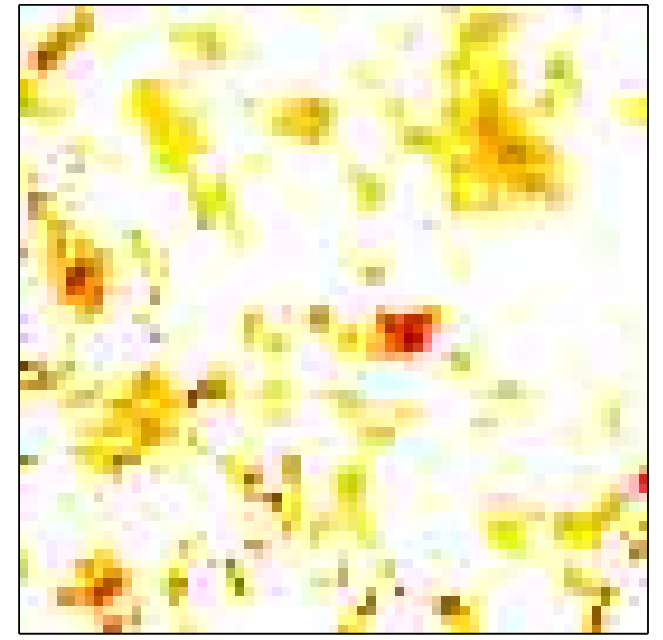

(a)

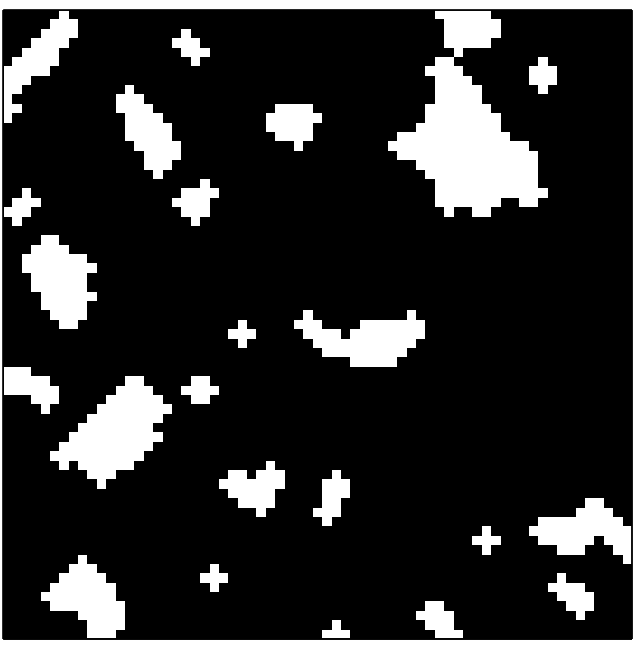

(c)

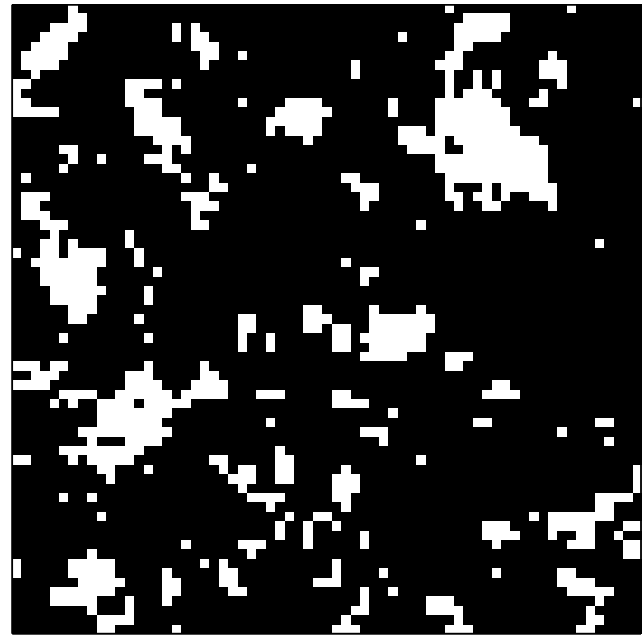

(b)

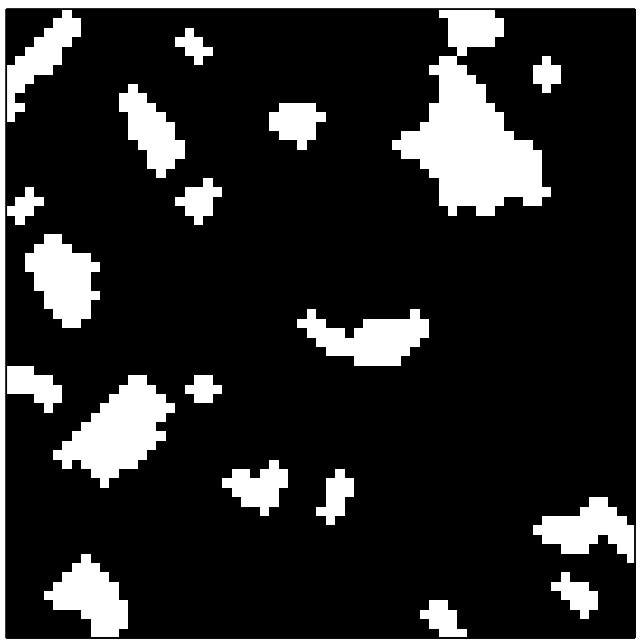

(d)

Figure 6.47: Vortex detection procedure in the the middle plane of the tomo-PIV data: (a) colorplot of $Q / Q_{\mathrm{rms}}$ and binary fields after (b) thresholding, (c) closing and opening and (d) cleaning operations.

haviour could contaminate the conditional statistics computing the same vortex many times with different feature values. The median filtering of detection field just before thresholding was tested and attenuated, but did not eliminate, the undesired oversegmentation. So, in the vortex analysis, the watershed algorithm was not employed. The mitigation of agglomerated structures was made by the use of appropriate threshold value and the attenuation of noise in the conditional statistics by the extraction of structure properties in relation to its centre, given by the maximum value of detection function in the connected region. In the hypothesis of the connected region being composed by more than one vortex, the location of the maximum value takes into account the properties of the strongest vortex into statistics.

An extra condition was added to prevent lower intensity vortices to con- 


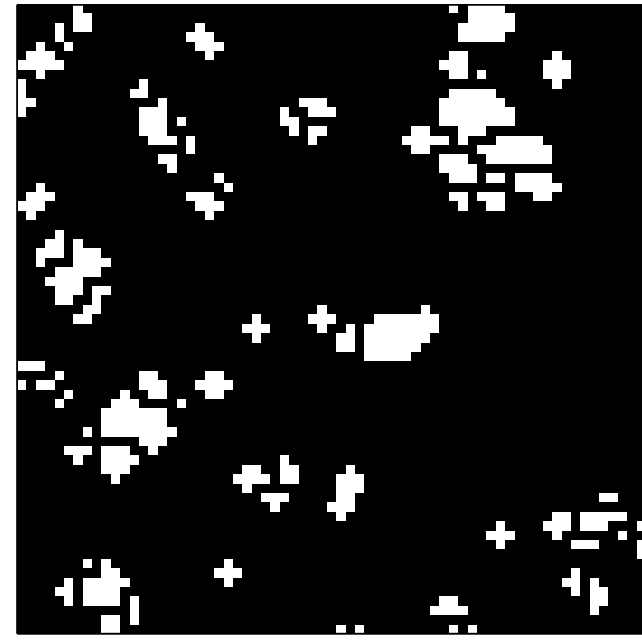

(a)

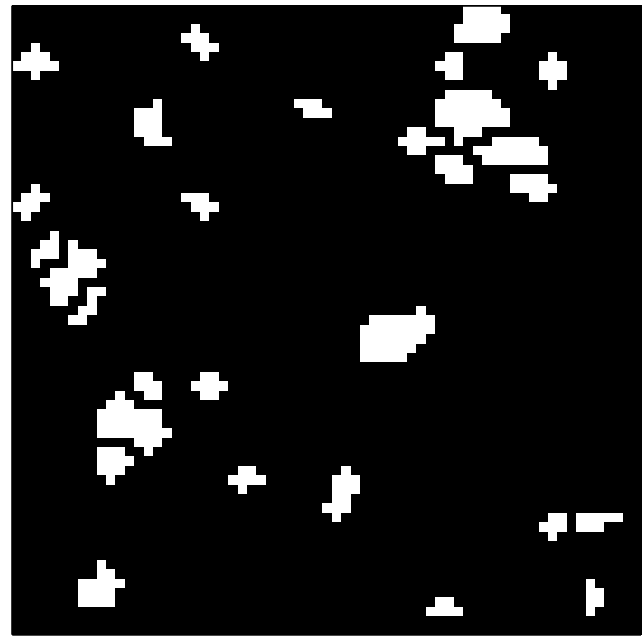

(b)

Figure 6.48: Vortex partitioning procedure in the the middle plane of the tomo-PIV data: binary field after (a) watershed and (b) cleaning operations.

tribute in the conditional statistics. Connected regions with three relatively weak vorticity components might result in $Q$ values that exceed the threshold and therefore would be identified as vortex cores by the algorithm. Removing these lower intensity vortices from the statistics reduce the uncertainty ([Honkan and Andreopoulos, 1997, Ganapathisubramani et al., 2006]) and slightly change the shape of the probability density distributions. Therefore, to include the vortex features in the statistics, the value of at least one component of fluctuating vorticity (computed in the vortex core) must be higher than $\alpha$ times the standard deviation of that component in the flow at the same wallnormal position, i.e. $\omega_{x}^{\prime}>\alpha \omega_{x \mathrm{rms}}^{\prime}\left(y^{+}\right)$or $\omega_{y}^{\prime} \alpha \omega_{y \mathrm{rms}}^{\prime}\left(y^{+}\right)$or $\omega_{z}^{\prime}>\alpha \omega_{z \mathrm{rms}}^{\prime}\left(y^{+}\right)$. In the present work, the relaxation factor $\alpha$ was assumed equal to one. Similar conditions were used by other researchers ([Ganapathisubramani et al., 2006, Gao et al., 2011]). At the end of the next section, a thorough discussion of the influence of the employed filters is presented.

These extra conditions can be inserted as an additional cleaning operation given by

$$
F_{B}= \begin{cases}0 & \text { if } F_{d}^{b} \leq \alpha \text { and } F_{d}^{c} \leq \alpha \text { and } F_{d}^{d} \leq \alpha \\ 1 & \text { otherwise }\end{cases}
$$

where the cleaning functions are defined for each vortex core as

$$
F_{d}^{b}=\frac{\omega_{x}^{\prime}}{\omega_{x \mathrm{rms}}^{\prime}\left(y^{+}\right)} \quad, \quad F_{d}^{c}=\frac{\omega_{y}^{\prime}}{\omega_{y \mathrm{rms}}^{\prime}\left(y^{+}\right)} \quad, \quad F_{d}^{d}=\frac{\omega_{z}^{\prime}}{\omega_{z \mathrm{rms}}^{\prime}\left(y^{+}\right)} .
$$

An additional condition, employing the 2D Q-criterion larger than zero, was tested to filter out vortices that have an axis aligned with the wall-normal- 

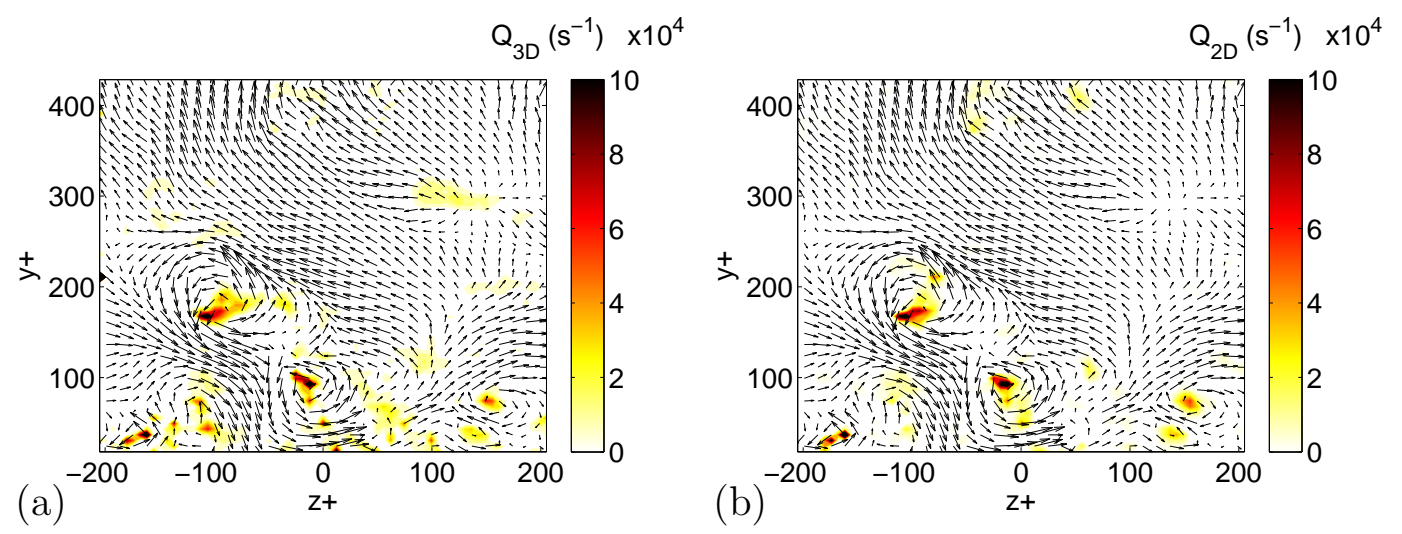

Figure 6.49: Comparison between the Q-criterion computed using (a) 9 and (b) 4 spatial derivatives. Vectors represents the fluctuating velocity field in the the middle plane of the tomo-PIV measurements.

spanwise plane. In the work of [Ganapathisubramani et al., 2006], they use similar filter to remove the inaccurate features of in-plane oriented vortices from the analysis. The 3D Q-criterion, based on the 9 spatial derivatives according to eq. (6.2.24), identifies vortices with all directions. Contrarily, the 2D Q-criterion, based only on the 4 spatial derivatives of velocity components in the plane, classify vortices that have some rotation in the plane.

$$
F_{B}= \begin{cases}0 & \text { if } Q_{2 D}>0 \\ 1 & \text { otherwise }\end{cases}
$$

Figure 6.49 shows the colormap of the 3D Q-criterion $\left(Q_{3 D}\right)$ and its lower dimensional $2 \mathrm{D}$ version $\left(Q_{2 D}\right)$ for the same tomo-PIV instantaneous measurements of figure 6.43. One over two fluctuating vectors are plotted in the same figure for clarity. Fewer vortex core regions are observed in the $Q_{2 D}$ field as compared to the $Q_{3 D}$. However, all intense regions in the $Q_{2 D}$ are present in the $Q_{3 D}$. Note that some discrepancies can be a result of the colormap code used since the 2D and 3D $Q$ computations produce different values.

Tests employing $Q_{2 D}>0$ favoured quasi-streamwise vortex in the conditional statistics. So this additional condition was not employed in the present analyses. Furthermore, as tomo-PIV is a three-dimensional technique, the noise in the features of in-plane oriented vortices experienced by [Ganapathisubramani et al., 2006] did not occur in the present data. The influence of the use of $Q_{2 D}>0$ filtering will be discussed along the text.

Therefore, for the vortex conditional statistical analyses that are presented in this section, the normalized Q-criterion detection function with a 0.5 threshold value was employed to classify vortices. After the cleaning operation based on structure areas and vorticity filtering, which eliminated about $50 \%$ of 
detected vortices, the total amount of structures remaining to compute statistics was about 25500, almost uniformly distributed in the investigation field and time.

Directly from the segmented binary field, geometric features can be extracted as height $\left(L_{y}\right)$ and width $\left(L_{z}\right)$ of the structure bounding box and area $\left(L_{y} \times L_{z}\right)$. The radius of the vortex core $(r)$ is computed for vortex with $0.9<L_{y} / L_{z}<1.1$, which avoid including the uncorrected agglutinated vortex into the statistics.

$$
r=\frac{L_{y}+L_{z}}{4}
$$

The distance in the measurement plane between two nearby structures can be computed. The distance is calculated as the minimum distance between the vortex centre, given by the maximum detection function within the connected region, and the vortex centre of its neighbours.

$$
\text { distance }=\min \left(\sqrt{\left(y_{c}-y_{i}\right)^{2}+\left(z_{c}+z_{i}\right)^{2}}\right),
$$

where $\left(y_{c}, z_{c}\right)$ is the vortex centre position and $\left(y_{i}, z_{i}\right)$ is the centre position of all $i$ vortices around the same wall-normal position at the same plane.

Also from the vortex core location, fluctuating velocity components, fluctuating vorticity components, Reynolds stresses and dissipation rates can be assessed.

In addition to the above mentioned quantities, the inclination angle of any individual vortex structure intersecting the measurement plane can be computed based on the orientation of the fluctuating vorticity vector at the vortex core, analogous of the orientation based on the fluctuating velocity components (figure 6.24). Projection and elevation angles are proposed in order to analyse the vortex orientation and the sense of rotation. Figure 6.50 depicts the elevation angle $\left(\theta_{e}\right)$ and projection angles with respect to the streamwise-wall-normal plane $\left(\theta_{x y}\right)$, streamwise-spanwise plane $\left(\theta_{x z}\right)$ and wallnormal-spanwise plane $\left(\theta_{y z}\right)$. This figure also presents the related quadrant definition for the projection angles based on the fluctuating vorticity.

These projection angles are defined as

$$
\begin{aligned}
& \theta_{x y}=\arccos \left(\frac{\omega_{x}^{\prime}}{\sqrt{\omega_{x}^{\prime 2}+\omega_{y}^{\prime 2}}}\right) \operatorname{sign}\left(\omega_{y}^{\prime}\right) \\
& \theta_{x z}=\arccos \left(\frac{\omega_{x}^{\prime}}{\sqrt{\omega_{x}^{\prime 2}+\omega_{z}^{\prime 2}}}\right) \operatorname{sign}\left(\omega_{z}^{\prime}\right) \\
& \theta_{y z}=\arccos \left(\frac{\omega_{y}^{\prime}}{\sqrt{\omega_{y}^{\prime 2}+\omega_{z}^{\prime 2}}}\right) \operatorname{sign}\left(\omega_{z}^{\prime}\right)
\end{aligned}
$$

The projection angles can assume values between $-180^{\circ}$ and $+180^{\circ}$. 

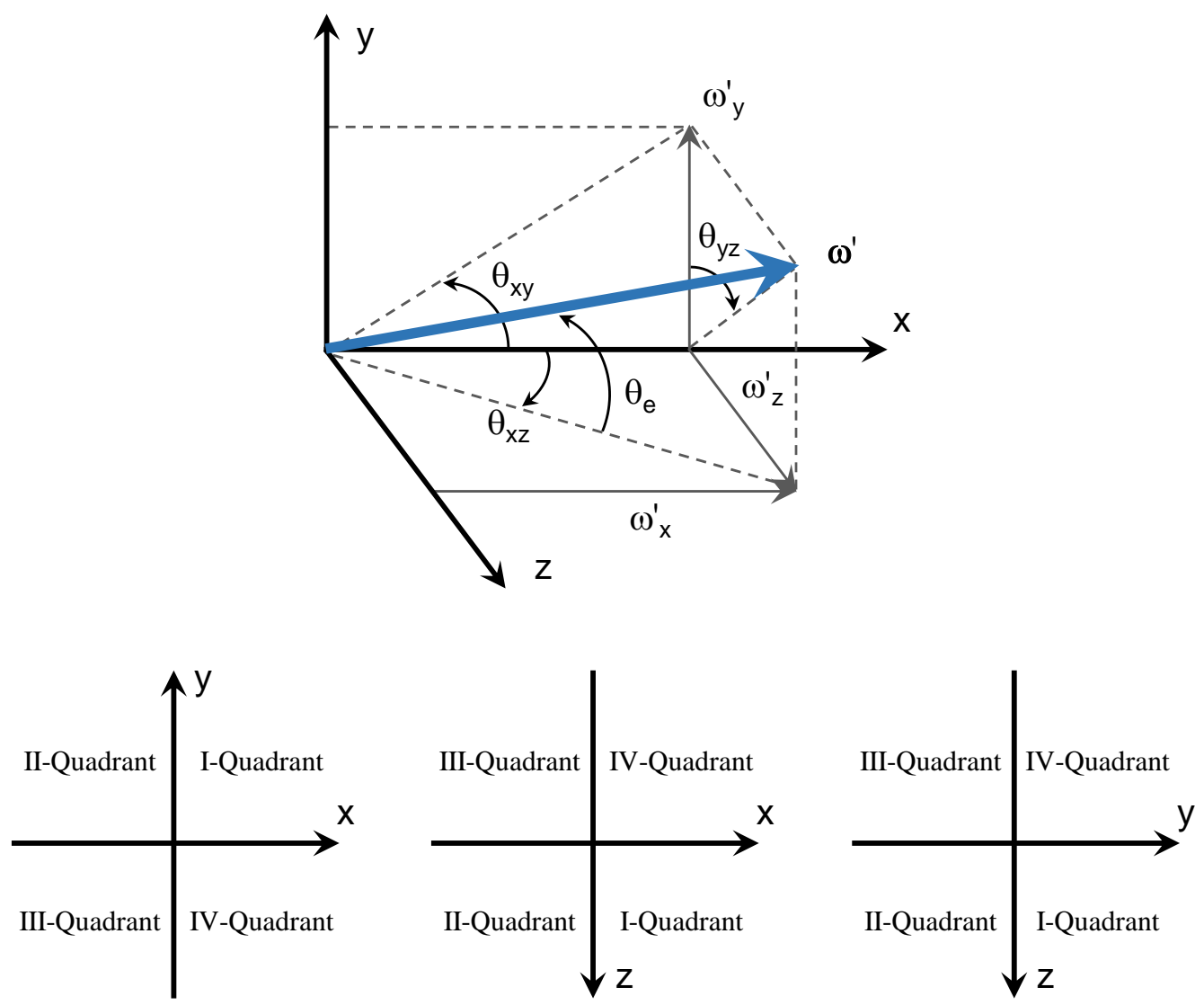

Figure 6.50: Elevation and projection angles based on the fluctuating vorticity vector and definition of quadrants respectively on streamwise-wall-normal, streamwise-spanwise and wall-normal-spanwise planes.

The elevation angle $\left(\theta_{e}\right)$ defines the angle between the vorticity vector and the wall (streamwise-spanwise plane) calculated by

$$
\theta_{e}=\arctan \left(\frac{\omega_{y}^{\prime}}{\sqrt{\omega_{x}^{\prime 2}+\omega_{z}^{\prime 2}}}\right),
$$

where the function arctan can assume values between $-90^{\circ}$ and $+90^{\circ}$.

Conditional sampling statistics of these vortex features can be computed with respect to wall-normal positions. Vortex structures that are around the same vortex core distance from the wall can be grouped to construct probability density functions, averages and standard deviations.

Vortex detection and feature extraction can be summarized as follows,

1. Detection of all regions with normalized 3D Q-criterion $\left(Q / Q_{\mathrm{rms}}\right)$ higher than a threshold.

2. Closing and opening operations to attenuate noise in the structure geometry. 

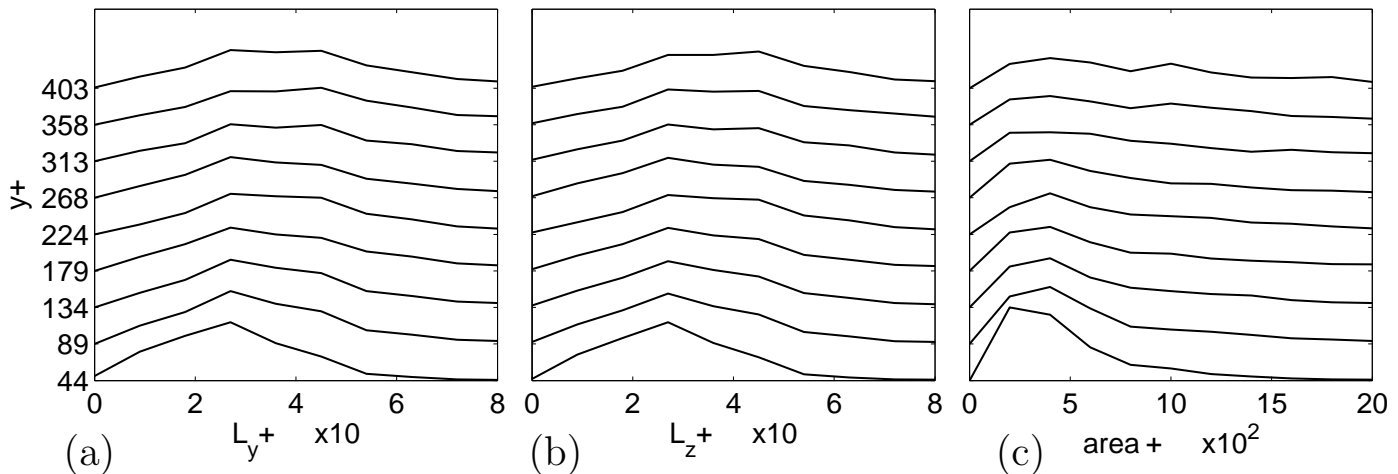

Figure 6.51: PDF of (a) height, (b) width and (c) area of turbulent structures for different wall-normal positions.

3. Cleaning operation to filter out small connected regions.

4. Elimination of vortices with all fluctuating vorticity components at the vortex core lower or equal than the standard deviation of that component computed at the same wall-normal position over the flow field.

5. Extraction of vortex features with respect to vortex centre (maximum $Q / Q_{\mathrm{rms}}$ inside structure).

6. Computation of conditional statistics of the various features for different wall-normal positions.

\section{Vortex statistics}

From the detected vortex structures, geometric features can be analysed. Figure 6.51 shows the PDF of height $\left(L_{y}\right)$, width $\left(L_{z}\right)$ and area, in wall units, of the cross-section of vortical structures on the wall-normal-spanwise plane as a function of the wall-normal distance. The mean and standard deviation of the dimensionless height, width and area increase along the wall-normal distance. Small oscillations are due to the limited amount of samples. It is important to mention that the size of turbulent structures depends on the threshold value adopted, so the size can slightly vary.

Following eq. (6.2.32), the radius $(r)$ of vortical structures can be computed using the width and height of the respective structure bounding box. Figure 6.52 shows the PDF of vortex radius as a function of the wall distance. The distributions in figure 6.52 show constant peaks at about 11 wall units, regarding the bin resolution adopted. The distribution seems to be log-normal with tails increasing with the distance from the wall.

Figure 6.53 presents the mean and standard deviation of the radius of vortices as a function of the wall-normal coordinate. The radii are scaled by 


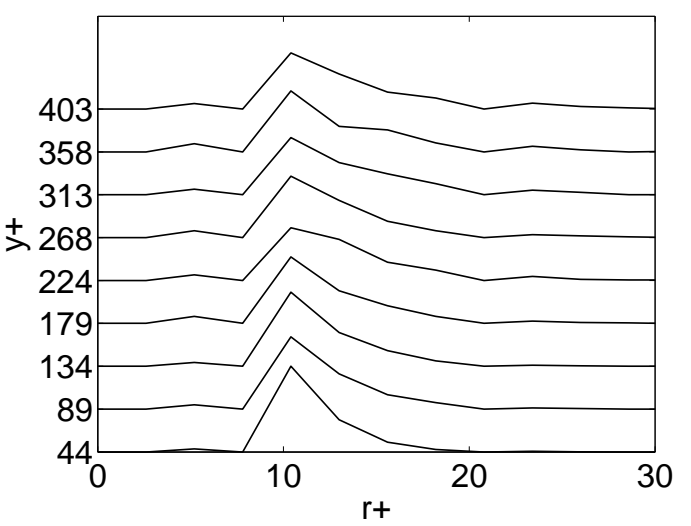

Figure 6.52: Probability density function of radius in function of wall-normal distance.

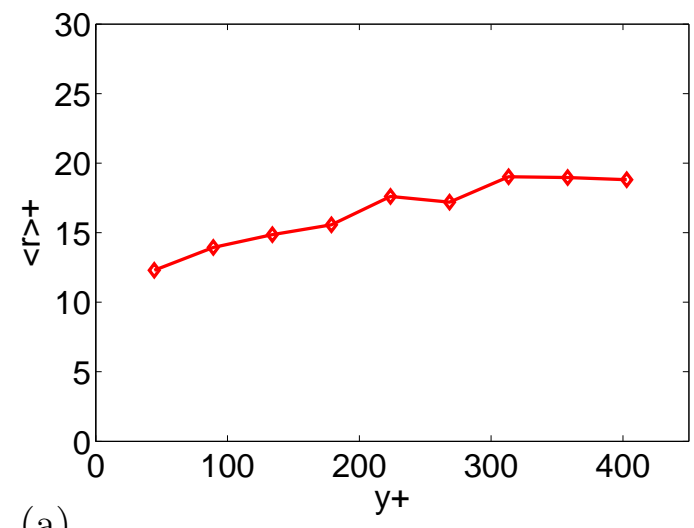

(a)

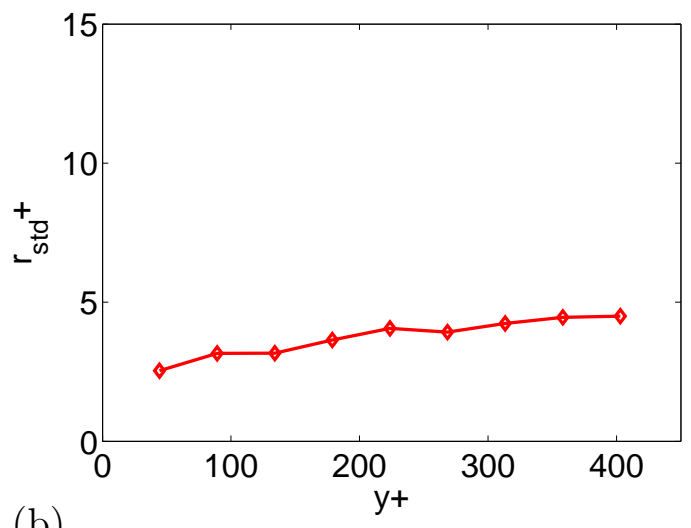

(b)

Figure 6.53: (a) Mean and (b) standard deviation of radius made dimensionless by inner variables for different wall-normal positions.

wall variables. On average, the dimensionless radii rise form about 12 to 20 and the standard deviation from 2 to 5 with the distance from the wall. These streamwise vortices have radii within the commonly range of 5 to 20 wall units ([Blackwelder and Eckelmann, 1979, Stanislas et al., 2008, Herpin et al., 2010, Herpin et al., 2013]). In the works of [Stanislas et al., 2008, Herpin et al., 2010, Herpin et al., 2013], vortex radii were obtained by fitting a theoretical Oseen vortex in the velocity data. Despite the different techniques employed, the results seem in excellent agreement with the present work. As mentioned before, small oscillations are attributed to the amount of samples available for the calculations and the size of the structure are threshold dependent, so it can somewhat vary. However, it should be mentioned that the mean vortex diameter was of the order of the interrogation volume and, consequently, some filtering effect is expected ([?, Herpin et al., 2008, Atkinson et al., 2014]). The increasing of vortex diameters with wall distance suggest a lift up of the vortices which is in line with [Lin et al., 2008].

Figure 6.54 displays the mean and standard deviation of the radius of vor- 


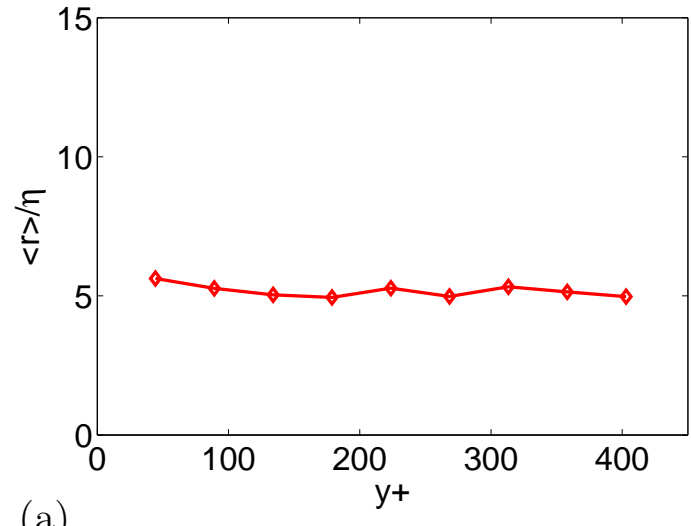

(a)

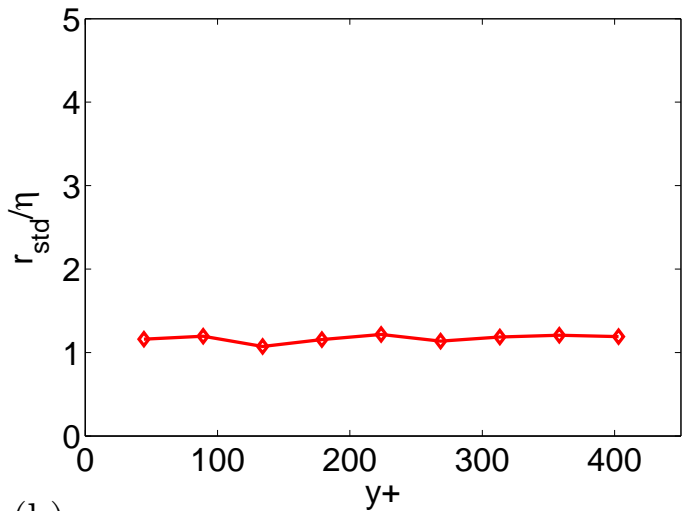

(b)

Figure 6.54: (a) Mean and (b) standard deviation of radius made dimensionless by Kolmogorov length for different wall-normal positions.

tices made dimensionless by the Kolmogorov length scale $\eta\left(y^{+}\right)$(figure 6.11). When scaled by the Kolmogorov length, vortices present constant mean radius of $5.2 \eta$ and standard deviations of $1.2 \eta$. These findings are in agreement with [Stanislas et al., 2008, Herpin et al., 2013], although the mean radius values of these authors were slightly higher. The discrepancy seems to be result of the threshold value to detect vortices and the estimated Kolmogorov length scale by the tomo-PIV technique.

Figure 6.55 shows the PDF of the minimum distance between vortices calculated from eq. (6.2.33). The PDFs are plotted with respect to the wallnormal position of vortex centre and the distances are in wall units. It can be observed from this figure that the probability density distribution becomes wider for increasing distances from the wall. The distances between vortex centres are mainly concentrated in a range from 25 to 125 wall units in the wall-normal-spanwise plane. To complement the vortex separation analysis, figure 6.56 presents the mean and standard deviation of the distance of vortex cores as a function of the wall-normal coordinate. The average distance between vortices is in the range of 60 to 80 wall units and the standard deviation between 20 and 40 wall units. These vortex separations are within the range of 50 to 100 wall units found in the literature ([Gupta et al., 1971, Blackwelder and Eckelmann, 1979, Kim et al., 1987]).

Another interesting vortex characteristic that can be extracted from the data can help to understand how vortical structures travel in the flow. The PDF of fluctuating velocity components at vortex centres with respect to the distance from the wall is presented in figure 6.57. The narrowest distributions are related to the wall-normal fluctuating velocity component and the widest distributions to the streamwise component, as expected for a near-wall turbulent flow. To clarify the vortical translation behaviour, the 


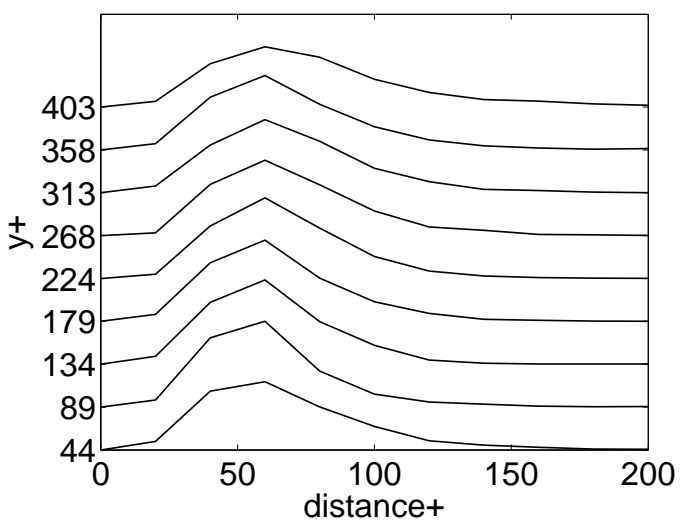

Figure 6.55: Probability density function of smallest distance between vortices as a function of wall-normal distance.
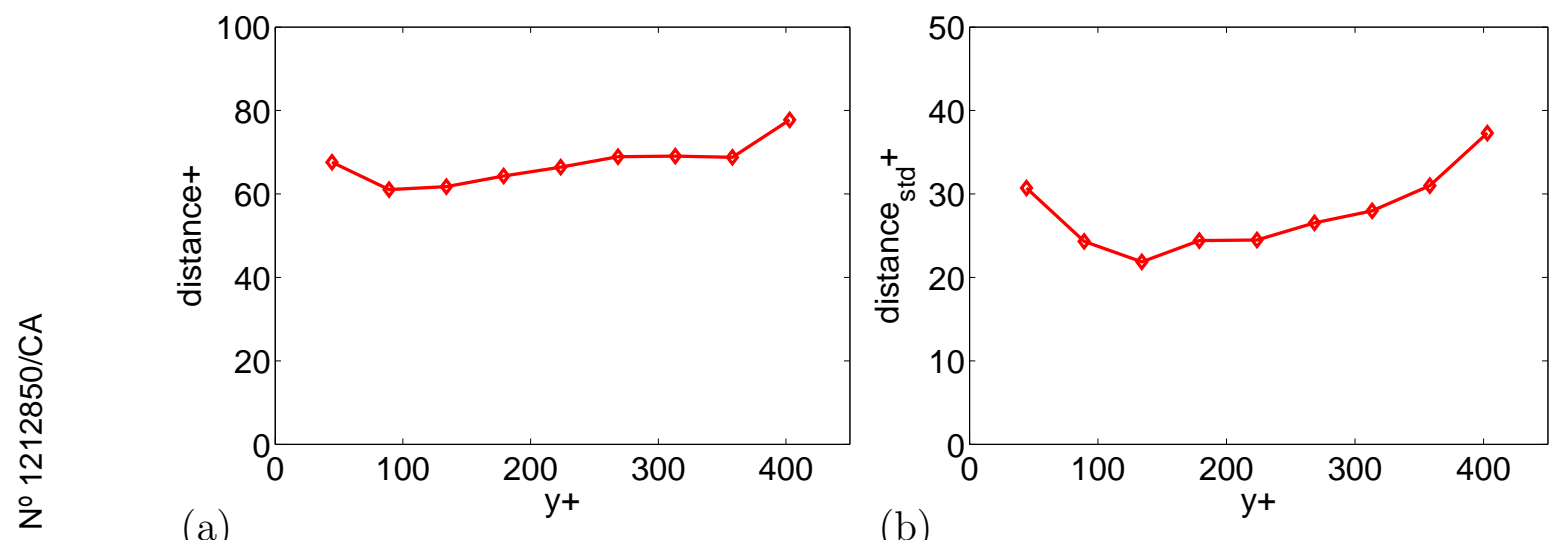

(b)

Figure 6.56: (a) Mean and (b) standard deviation of vortex separation for different wall-normal positions.

mean and standard deviation of fluctuating velocity components as a function of wall positions are presented in figures 6.58(a) and 6.58(b).

Figure 6.58(a) shows zero mean wall-normal and spanwise fluctuating velocities concerning the prevailing levels of expected uncertainties. Although the average streamwise fluctuating velocity appears slightly under zero, the values are of the same order as the uncertainty and some oscillations signal a lack of convergence. The convergence of the streamwise fluctuating velocity is harder than the others since this measurement component presents higher standard deviation (see figure 6.58(b)). [Carlier and Stanislas, 2005] and [Herpin et al., 2013] also found the streamwise velocity of vortical structures slightly under the flow velocity. They concluded that the differences were negligible. Consequently, the present results shows that, on average, the turbulent structures are convected with approximately the local flow velocity, which is in agreement with the literature ([Adrian et al., 2000a, Christensen and Adrian, 2002, Carlier and Stanislas, 2005, Wu and Christensen, 2006, Herpin et al., 2010, Herpin et al., 2013]). 


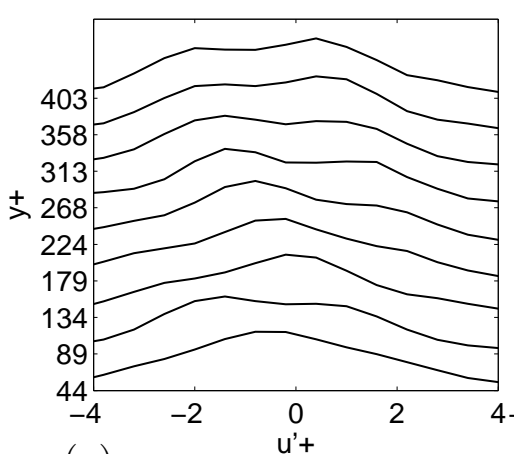

(a)

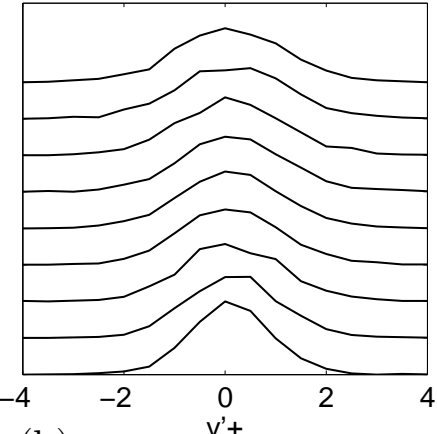

(b)

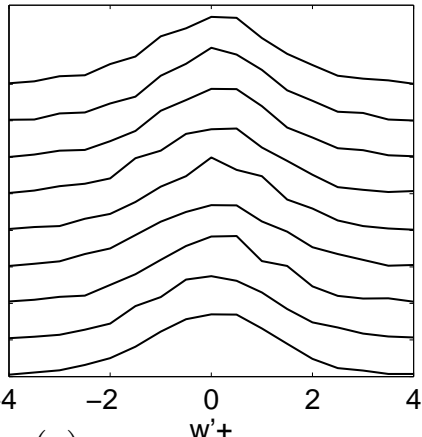

(c)

Figure 6.57: PDF of fluctuating (a) streamwise, (b) wall-normal and (c) spanwise velocities of the vortices centroid for different wall-normal positions.

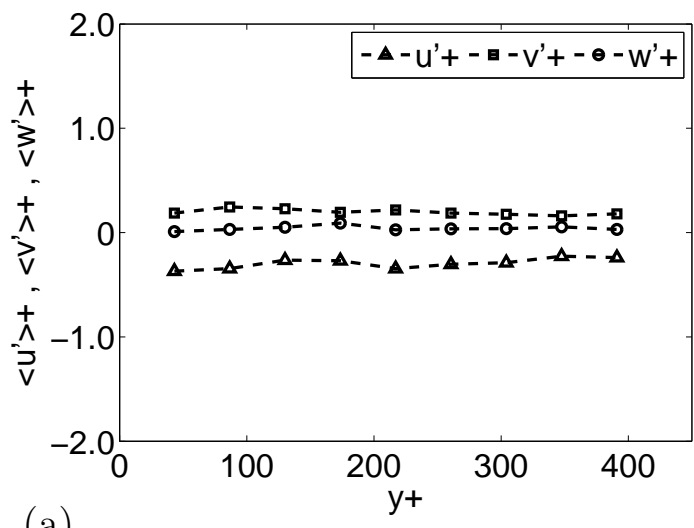

(a)

Figure 6.58: (a) Mean and components of vortex population for different wall-normal positions. Red diamond refers to flow statistics data, while dashed black lines to vortex conditional statistics.

In figure 6.58(b), the standard deviations of fluctuating velocity components of the vortices coincide with the global flow statistics curves, revealing no discrepancies between vortices and the flow with respect to these quantities. These findings are in line with [Wu and Christensen, 2006], which analysed the dispersion of streamwise velocity of vortices detected in the streamwisewall-normal plane for a channel flow at $R e_{\tau}=1760$ and a boundary layer at $R e_{\tau}=2350$, and also with [Herpin et al., 2013], which studied the dispersion of streamwise velocity of vortices detected in the wall-normal-spanwise plane of a boundary layer at various $R_{\theta}$, from 7630 to 18950 .

The Reynolds stresses, calculated in the core of vortex structures, are presented in figure 6.59(a) with respect to wall-normal position. The Reynolds stresses from the complete flow statistics is also plotted in the figure for comparison. This conditional statistics agrees well with the global flow statistics. Slight oscillations and discrepancies might be linked to the amount of vortices used to compute the statistics. This results lead to the conclusion that vortices 


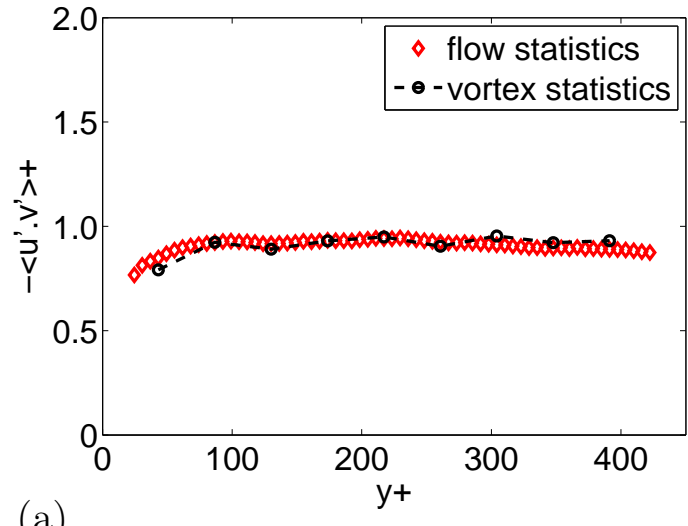

(a)

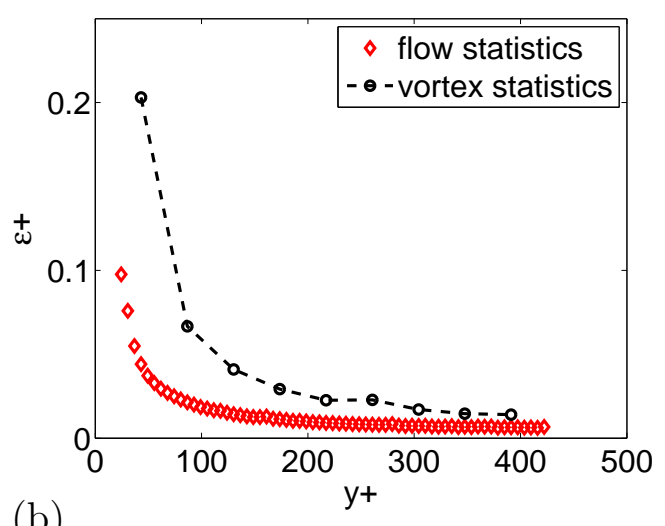

(b)

Figure 6.59: (a) Reynold stresses and (b) dissipation rate of vortex centres in relation to wall-normal distance.

are not directly linked to the turbulent production, although they can induce other events related to production such as ejection and sweeps ([Robinson, 1991b, Lin, 2006]).

Figure 6.59(b) compares the conditional dissipation rate of vortex structures with the entire flow for different wall-normal positions. Conditional statistics present dissipation distributions over those for the complete flow statistics. Higher dissipation rates are observed close to the wall, where the most intensity vortices were detected. The presence of stronger vortices in the near wall region was reported in the literature ([Robinson, 1991a, Stanislas et al., 2008]). The vortex contribution on the total dissipation rate was (on average) $25 \%$ with a relative structure area of only $10 \%$ of the total flow area.

Figure 6.60 shows the probability density function of the fluctuating vorticity components $\left(\omega_{x}^{\prime}, \omega_{y}^{\prime}, \omega_{z}^{\prime}\right)$ and its magnitude $\left(\left|\omega^{\prime}\right|\right)$, scaled by inner variables, with respect to the wall-normal position. The fluctuating streamwise vorticity clearly shows a bimodal distribution, with peaks at about -0.25 and 0.25 that increase in intensity with increasing distance from the wall. The peak locations and the spreading of the fluctuating vorticity component distributions are linked to the Q-criterion threshold employed. For smaller threshold values, the peaks of the fluctuating streamwise vorticity distribution get closer and the distribution tails increase, although the behaviour of quasisymmetric bimodal distribution remains. This bimodal distribution is related to the homogeneity of almost equal number of clockwise (49\%) and counterclockwise $(51 \%)$ vortices. Clockwise vortices are defined here as vortices with a positive fluctuating streamwise vorticity at the vortex core, and counterclockwise the ones presenting a negative fluctuating streamwise vorticity. Near the wall the distributions are spread out revealing the occurrence of all types of vortices. The mean and mode of the fluctuating wall-normal and spanwise 


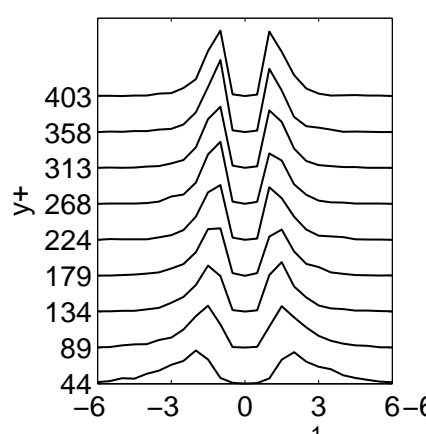

(a) $\omega_{x}^{\prime} \times 10^{-1}$

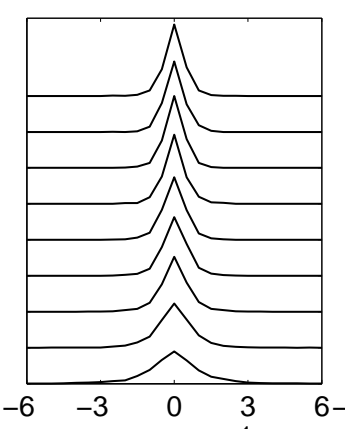

(b) $\omega_{y^{+}}^{\prime} \times 10^{-1}$

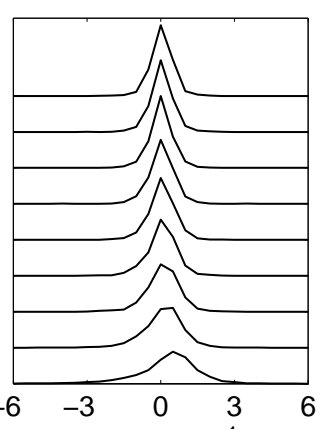

(c) $\omega_{z}^{\prime}+\times 10^{-1}$

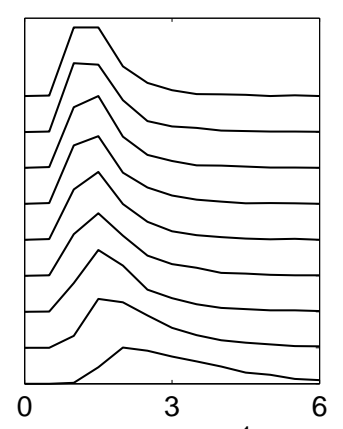

(d) $\left|\omega^{\prime}\right|+\times 10^{-1}$

Figure 6.60: PDF of fluctuating (a) streamwise, (b) wall-normal and (c) spanwise vorticities and (d) magnitude of the vorticity vector at the centres of vortical structures for different wall-normal positions.

vorticity distributions are zero. The PDF of figure 6.60(d) shows the peak of the magnitude of fluctuating vorticity moving to the left with the distance from the wall. As mentioned previously, the vortices are less intense with the increase of wall distance mainly due to the detection function employed.

Figure 6.61 presents the root-mean-square of fluctuating vorticity components employing conditional statistics of vortex centres. The root-meansquare of fluctuating vorticity of the entire flow is also plotted for comparison. The plot is made dimensionless by inner variables. The fluctuating vorticity components of vortex structures are significantly above those of the flow statistics, as expected. It is important to observe that the streamwise component of vortex structures is markedly above other fluctuating vorticity components. Indeed, the streamwise vorticity component contributed to $25 \%$ (on average) to the total root-mean-square of streamwise fluctuating vorticity of the flow, while wall-normal vortex fluctuating vorticity and spanwise vortex fluctuating vorticity were responsible, each one, to about $19 \%$ of the total. This discrepancy seems to be result of the higher presence of quasi-streamwise vortices, that can be a result of the vortex filtering due to the orientation of the measurement volume as will be discussed afterwards in this section.

From the fluctuating vorticity vector at the vortex centre, the orientation of vortical structures can be computed by eq. (6.2.34) to (6.2.37). Figure 6.62 shows the probability density function of projection angles onto the streamwise-wall-normal plane $\left(\theta_{x y}\right)$, streamwise-spanwise plane $\left(\theta_{x z}\right)$ and wallnormal-spanwise plane $\left(\theta_{y z}\right)$ of the vortex core detected in the measured wallnormal-spanwise plane for various distances from the wall. For the plots of figures 6.62 to 6.64 , the additional cleaning condition based on the fluctuating vorticity components, eq. (6.2.29), was not employed since it would reduce drastically the amount of structures, as mentioned previously, and, as a 


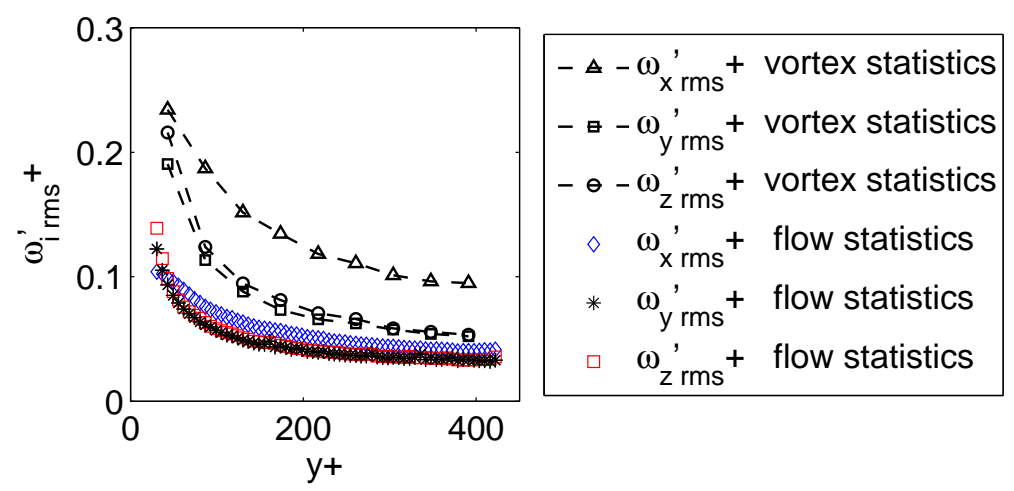

Figure 6.61: Root-mean-square of streamwise, wall-normal and spanwise vorticities computed for conditional and flow statistics in relation to the distance to the wall.
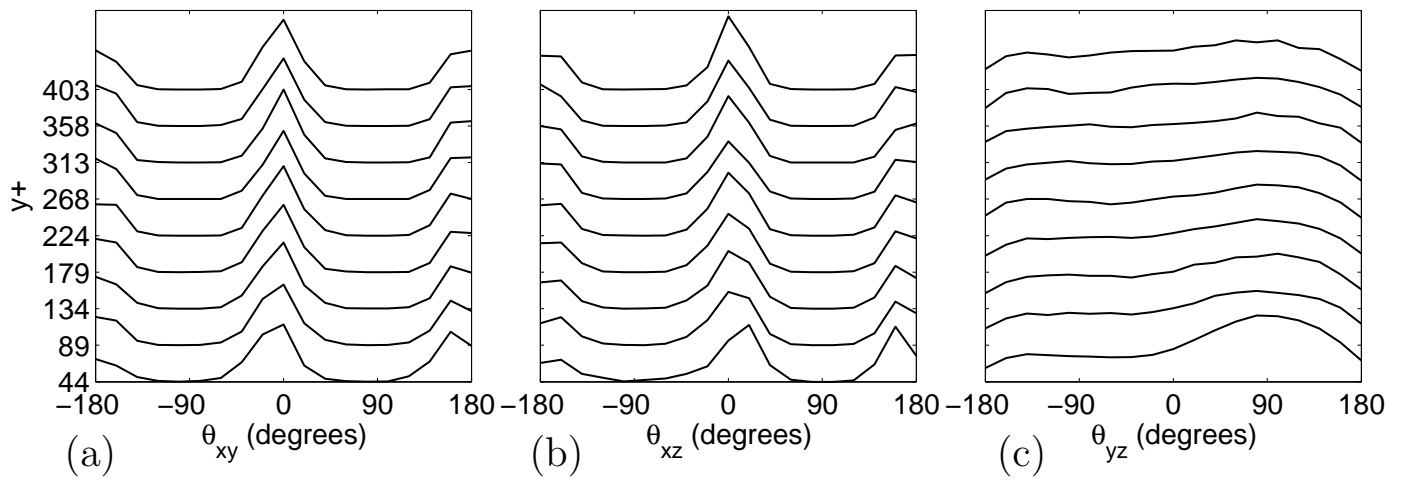

Figure 6.62: Probability density function of projection angles onto the (a) streamwise-wall-normal plane, (b) streamwise-spanwise plane and (c) wallnormal-spanwise plane of vortex core for different wall-normal positions.

consequence, the conditional statistics of vortex angles would not be acceptably converged. All preceding vortex analyses were done adopting this filter. Regarding figure $6.62(\mathrm{a})$ and (b), most of the vortices display zero or $180^{\circ}$ inclination when projected on the streamwise-wall-normal and streamwisespanwise planes. These inclinations are an indication of the clock-wise and counter-clock-wise streamwise vortices. Figure 6.62(c) shows almost an uniform probability density distribution of vortex inclinations projected onto the wall-normal-spanwise plane. For $y^{+}<200$ a peak in the $90^{\circ}$ is observed, although it seems to be caused by lack of convergence.

Figure 6.63 shows the probability density function of the elevation angle $\left(\theta_{e}\right)$, defined as the angle between the fluctuating vorticity vector at the vortex core and the wall, for various wall-normal positions. The distributions present a relatively similar shape with just one peak at zero. About $95 \%$ of the vortex inclinations are concentrated between $-40^{\circ}$ and $40^{\circ}$.

The average vortex elevation angle $\left(\left\langle\theta_{e}\right\rangle\right)$ and the standard deviation of this angle $\left(\theta_{e s t d}\right)$ can be observed in figure 6.64 with respect to wall-normal 


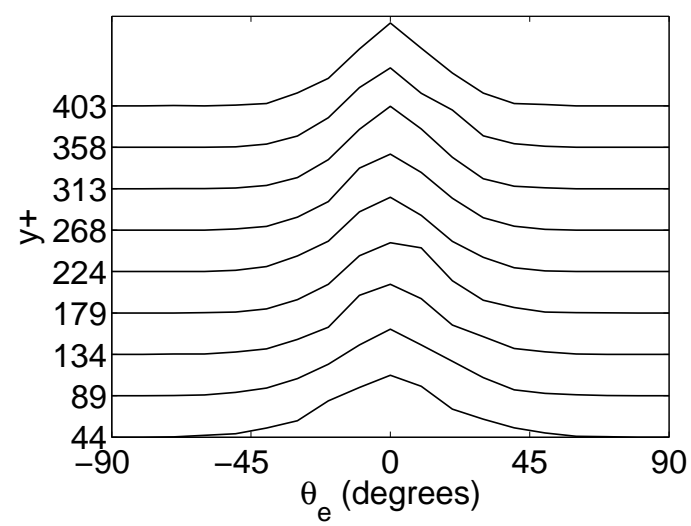

Figure 6.63: Probability density function of elevation angles of vortex core for different wall-normal positions.
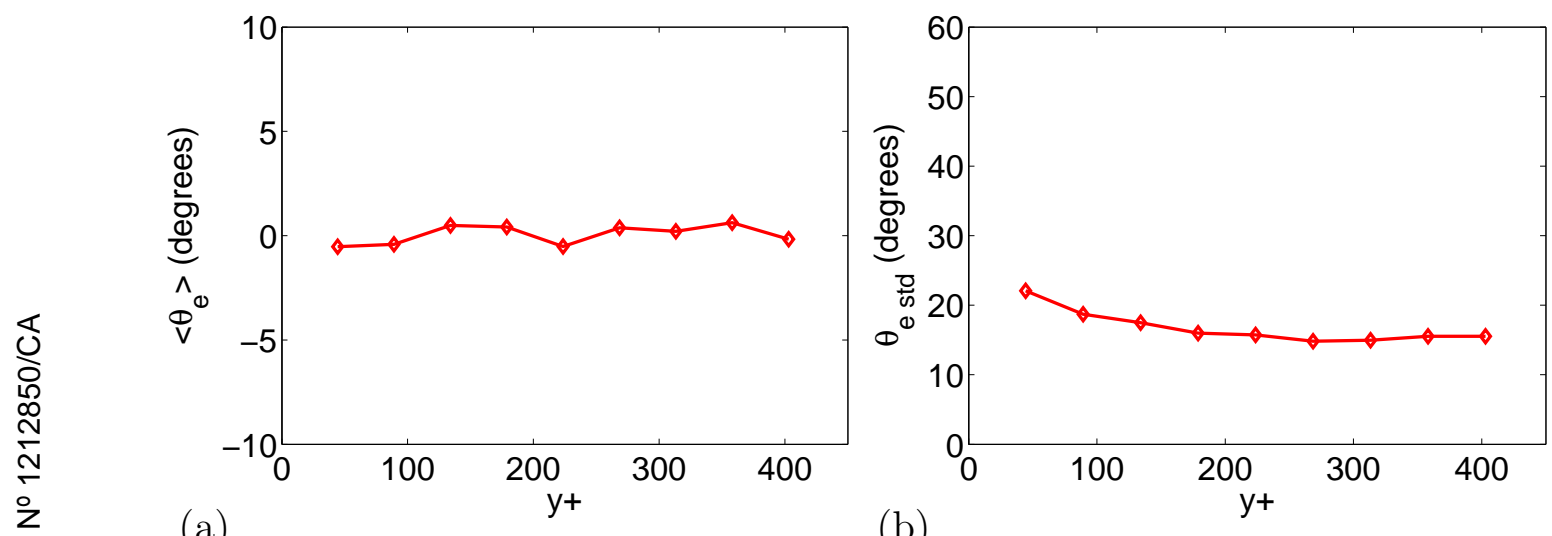

(a)

Figure 6.64: (a) Mean and (b) standard deviation of vortex streamwise inclination for different wall-normal positions.

distance. As can be seen by the plots, the vortical structures are, in average, aligned with the streamwise direction and present a standard deviation of about $20^{\circ}$. These findings are not in agreement with the variety of inclinations that can be found in the visualizations observed in the literature [Scarano and Poelma, 2009, Atkinson et al., 2011, Schröder et al., 2011, Foucaut et al., 2011a, Jodai et al., 2014]. The vortex statistics just presented are related to the quasi-streamwise vortices that populate the turbulent structures detected. The detection procedure and measurement-plane orientation affect the sensibility of detection of some kinds of vortices in the whole vortex population. As no significant variation is observed in the PDFs of projection angles within the logarithmic region, the probability density function can be computed using all vortices that belong to that region in order to better converge vortex conditional statistics and understand the filtering effects mentioned.

Figure 6.65 shows a comparison of the projection angle on the streamwisewall-normal plane $\left(\theta_{x y}\right)$ of [Honkan and Andreopoulos, 1997] data at $y^{+}=12.5$ with the present tomo-PIV vortex data for the logarithmic region $(100<$ 


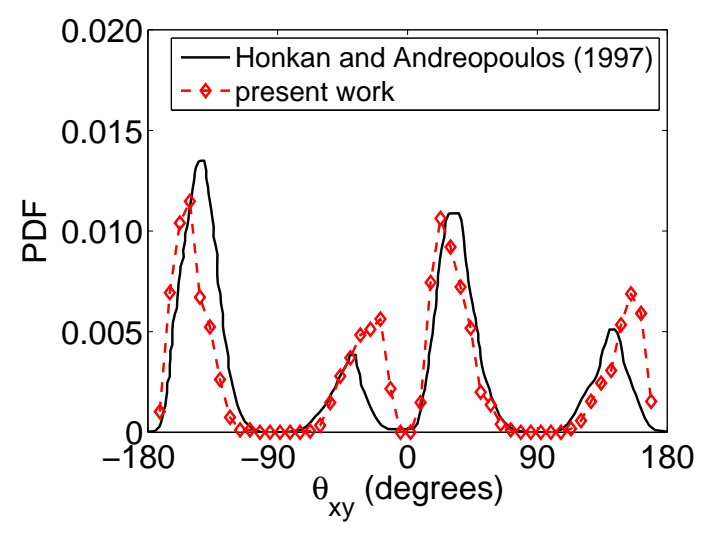

Figure 6.65: Probability density function of projection angle on the streamwisewall-normal plane for [Honkan and Andreopoulos, 1997] and vortex conditional statistics of tomo-PIV data.

$\left.y^{+}<500\right)$. The additional filtering condition based on the fluctuating vorticity components, eq. (6.2.29), was employed in the analysis of this figure. [Honkan and Andreopoulos, 1997] used a 9-sensor hot-wire anemometer probe to assess the full velocity gradient tensor in the near-wall region of a windtunnel turbulent boundary layer with $R e_{\theta}=2790$. A good agreement in the inclination angle distributions of figure 6.65 is obtained between these two studies. Both PDFs show four preferable orientations. The [Honkan and Andreopoulos, 1997] data display two primary peaks approximately at $35^{\circ}$ and $-140^{\circ}$, which are about $180^{\circ}$ apart, and two secondary peaks approximately at $-35^{\circ}$ and $140^{\circ}$, while the present tomo-PIV work shows correspondent peaks at about $25^{\circ},-150^{\circ},-20^{\circ}$ and $155^{\circ}$, respectively. The primary peaks are associated with structures carrying either positive or negative $\omega_{x}^{\prime}$ in the first and third quadrants (please see figure 6.50). These primary peaks seems to be related to hairpin-like vortices as suggested by [Honkan and Andreopoulos, 1997]. The angles are in agreement with [Smith and Walker, 1995] that described the inclination of a hairpin-like vortex as varying from $10^{\circ}$ in the most upstream part of the legs to $45^{\circ}$ in the lifted part of the legs or to more than $45^{\circ}$ at the head of the vortex. The discrepancies between the two distributions in figure 6.65 can be attributed mainly to the distinct wall-normal positions and different methods employed.

In order to elucidate the influence of the in-plane vortex filter, the work of [Ganapathisubramani et al., 2006] is presented. Figure 6.66 shows the elevation angle $\left(\theta_{e}\right)$ of [Ganapathisubramani et al., 2006] data at $y^{+}=110$. [Ganapathisubramani et al., 2006] performed a dual-streamwise-spanwiseplane PIV to compute the full velocity gradient tensor in the near-wall region of a wind-tunnel turbulent boundary layer at $R e_{\theta}=2800$. [Ganapathisubramani et al., 2006] studied vortex orientation based on the vorticity vector and 


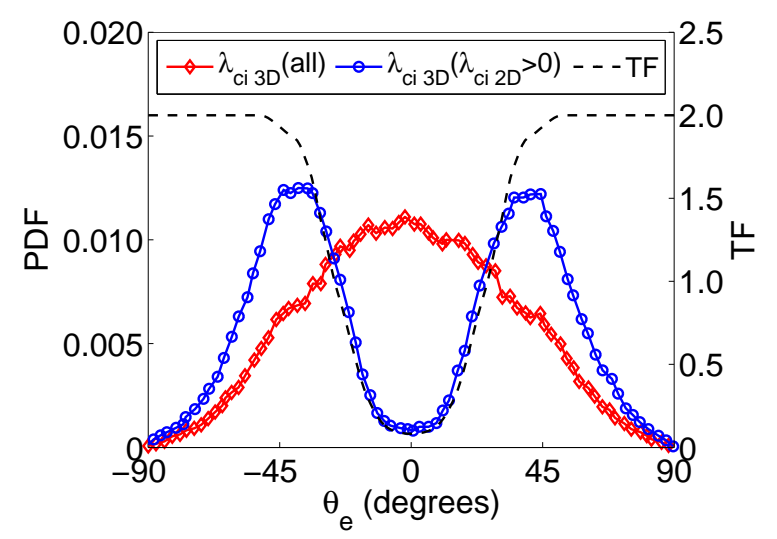

Figure 6.66: Probability density function of elevation angle of unfiltered and filtered vortices detected on the streamwise-spanwise PIV plane at $y^{+}=110$ (adapted from figure 7 of [Ganapathisubramani et al., 2006]). The axis of the transfer function $(\mathrm{TF})$ reads on the right.

employed similar procedure of the present work to extract vortices. The differences between the present work and their study were mainly in the use of the $3 \mathrm{D}$ swirling strength criterion $\left(\lambda_{c i 3 D}\right)$ as a detection function, a growing algorithm to segment vortices and the orientation of the investigation plane. In figure 6.66, the probability density function of elevation angles computed before and after filtering vortices with a in-plane orientation are presented. [Ganapathisubramani et al., 2006] employed the 2D swirling strength criterion $\left(\lambda_{c i 2 D}>0\right)$ to remove vortices aligned with the measuring plane. The transfer functions (TF) of the probability density functions employing this $2 \mathrm{D}$ filter is plotted in the same figure 6.66 using the axis on the right. The TF was calculated dividing point by point the PDF of elevation angle computed with all vectors by the PDF constructed with the vectors filtered by $\lambda_{c i 2 D}>0$. In order to remove noise oscillations in the $\mathrm{TF}$ a 5-neighbour moving-average filter was passed previously to the TF calculation in the PDFs. The TF shows a symmetrical band-cut filter that attenuates angles between $-27^{\circ}$ and $27^{\circ}$ and amplifies others with a maximum amplification factor of $2 \mathrm{x}$. The filtered PDF shows a distribution with two peaks, one at $-45^{\circ}$ and other at $45^{\circ}$, which were obscured in the distribution before filtering. The peaks are related to the legs of hairpin-like vortices with negative and positive fluctuating streamwise vorticity, respectively ([Ganapathisubramani et al., 2006]). The 2D filter was able to proper filter vortices aligned with the wall in order to obtain inclination statistics with two peaks that can be related to quasi-symmetrical hairpin vortices ([Adrian et al., 2000b]) or cane vortices ([Carlier and Stanislas, 2005, Herpin et al., 2013]).

The in-plane vortex filter just presented performs completely different when applied to other plane orientation. The present work uses a $Q_{2 D}>0$ 


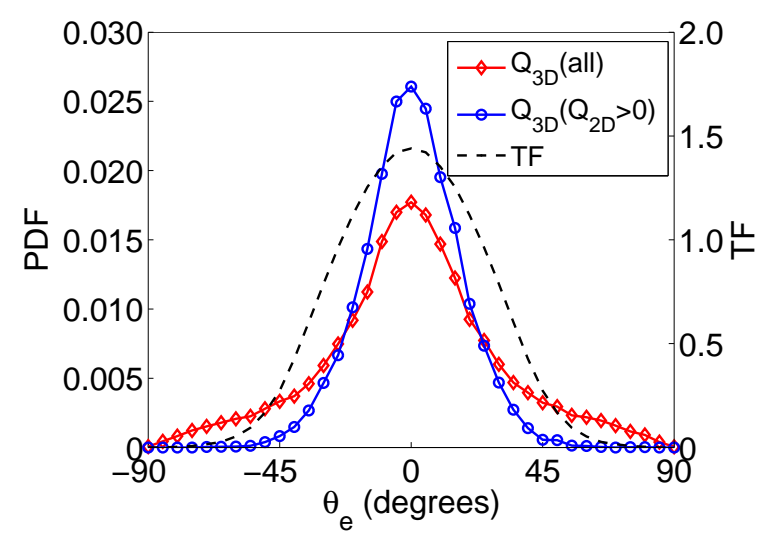

Figure 6.67: Probability density function of elevation angle of unfiltered and filtered vortices detected on the wall-normal-spanwise plane at $100<y^{+}<500$ at the middle plane of tomo-PIV data. The axis of the transfer fuction (TF) reads on the right.

criterion to filter the vortices aligned to the plane according to eq. (6.2.31), similar to the $\lambda_{c i 2 D}>0$ filter of [Ganapathisubramani et al., 2006] discussed in the previous paragraph. Figure 6.67 shows the probability density function of the elevation angle $\left(\theta_{e}\right)$ of the present tomo-PIV data before and after $Q_{2 D}>0$ filter for vortices located at $100<y^{+}<500$. The transfer functions (TF) of this in-plane filter, calculated by the same procedure explained previously, is plotted in the same figure 6.67 using the axis on the right. As can be observed, the unfiltered PDF, $Q_{3 D}$ (all), detects more vortices aligned with respect to the wall than the correspondent unfiltered PDF of figure 6.66, $\lambda_{c i 3 D}$ (all). This fact seems to be a consequence of the orientation of the investigation plane, since the wall-normal-spanwise plane is more sensitive to detect vortices with lower values of $\theta_{e}$. The orientation of the plane of measurements also determines the transfer function of the $2 \mathrm{D}$ vortex detection filter. The TF applied to the streamwise-spanwise plane removes vortices with elevation angles close to zero, while the TF applied to the wall-normal-spanwise plane removes vortices with high elevation angle magnitude. The TF of figure 6.67 is a symmetrical band-pass filter that amplifies angles between $-23^{\circ}$ and $23^{\circ}$ with a maximum amplification factor of $1.5 \mathrm{x}$ and attenuates others. Although the $Q_{2 D}>0$ criterion applied in the present work filters undesired vortices aligned with the plane, the large amount of quasi-streamwise vortices outweighs the hairpinlike vortices. So the orientation of the volume employed in the present study is adequate to compute vortex conditional statistics of quasi-streamwise-oriented vortices rather than hairpin-like ones.

In order to detect hairpin-like vortices (or at least increase their relative occurrence comparing to other vortices, since the frequency of occurrence hairpin-like structures is small according to [Robinson, 1991a] and [Sheng et al., 
2008]), the filter based on the fluctuating vorticity components ([Honkan and Andreopoulos, 1997]) was employed again for the elevation angle, eq. (6.2.29). Figure 6.68 shows the unfiltered PDF of figure 6.67 and the PDF after fluctuating vorticity filtering, called $\omega_{i}^{\prime}>\alpha \omega_{i \mathrm{rms}}^{\prime}$ with $\alpha=1$. The transfer function (TF) was calculated as defined above and is plotted for clarity in the same figure using the axis on the right. The TF is a symmetrical two peak function, with peaks at $-35^{\circ}$ and $35^{\circ}$, that amplifies angles between $-55^{\circ}$ and $-15^{\circ}$ and between $15^{\circ}$ and $55^{\circ}$ with a maximum amplification factor of $2.3 \mathrm{x}$ and attenuates other angles. In this case, the filter is able to attenuate the influence of quasi-streamwise vortices. Wall-normal and spanwise vortices, which would be removed employing $Q_{2 D}>0$ in a wall-normal-spanwise measurement plane, are removed by this filter, since they have lower $\omega_{y}^{\prime}$ and $\omega_{z}^{\prime}$ values, respectively. In the filtered PDF distribution, two peaks appear: one at about $-30^{\circ}$ and other at $30^{\circ}$. The filter was able to reveal two shoulders, one at $-45^{\circ}$ and other at $45^{\circ}$, in the distribution that were previously hidden in the unfiltered PDF. A closer observation of the results of figure 6.68 shows that the primary peaks at $\pm 30^{\circ}$ are induced by the TF filter and seem to be non-physical. However, one can observe two shoulders at $\pm 45^{\circ}$ in the filtered PDF of the same figure, $Q_{3 D}\left(\omega_{i}^{\prime}>\omega_{i \mathrm{rms}}^{\prime}\right)$, related to the two peaks of the filtered PDF in figure 6.66, $\lambda_{c i 3 D}\left(\lambda_{c i 2 D}>0\right)$. The inclination of $\pm 45^{\circ}$ seems to represent the physics of the lifted part of the legs of the hairpin-like vortices. This value might be contaminated in our measurements due to the use of vortices belonging to the entire logarithmic region to converge statistics. Filtering effect is also relevant in these results. If, instead of applying a relaxation factor $\alpha$ equal to one in eq. (6.2.29) other value was used, the peaks of the TF would change and so would the peaks in the filtered PDF distribution. Contrarily of what was employed in the present work, the value of $\alpha$ could be increased by a factor of two or three until the two peaks at $\pm 45^{\circ}$. However, this procedure seemed as excessive data filtering. It is very interesting to observe how the application of filters to the data changed the most probable values of detected features, in the present case, vortex inclinations.

\subsubsection{Summary of Flow Structure Statistics}

Turbulent structure statistics, namely, low- and high-speed regions, ejections, sweeps and vortices, were computed by means of conditional sampling applying normalized detection functions to account for flow quantity changes along the wall-normal direction. The middle plane of the tomo-PIV data, containing all spatial derivatives, was analysed to study the near-wall structures of a boundary-layer flow with $R e_{\theta}$ of 8500 inside a wind tunnel. 


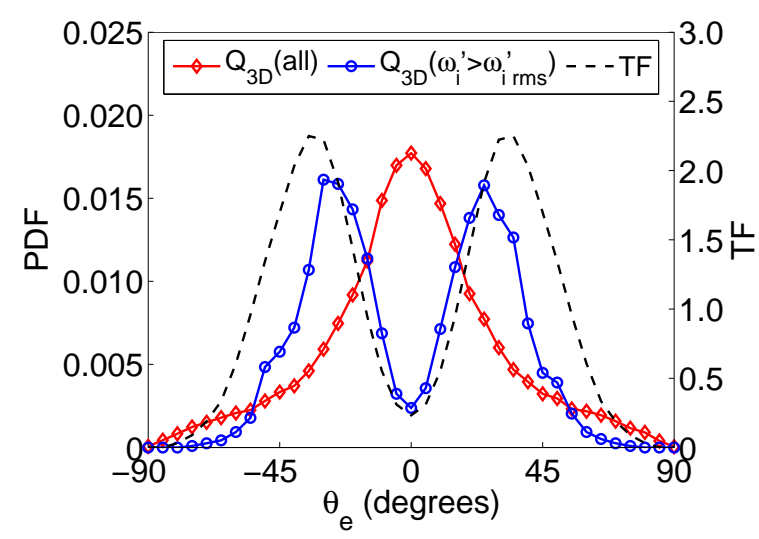

Figure 6.68: Probability density function of elevation angle of unfiltered and filtered vortices detected on the wall-normal-spanwise plane at $100<y^{+}<500$ at the middle plane of tomo-PIV data. The axis of the transfer fuction (TF) reads on the right.

The low- and high-speed conditional statistics revealed that, in average, the low-speed regions moved upwards and the high-speed regions towards the wall. These findings were in agreement with the literature and indicates the strong influence of ejection and sweep events in the mentioned structures. Contrarily, the average of fluctuating velocity components at vortex structures were zero, leading to the conclusion that vortices are, in average, convected at the local flow velocity.

Regarding the turbulent fluctuations, low- and high-speed regions, ejections and sweeps display higher root-mean-square values of the fluctuating streamwise component than the neighbouring fluid. The ejection contribution to the total root-mean-square of fluctuating streamwise velocity was, on average, $20 \%$ with a relative structure area of about $17 \%$ of the total flow area. Sweeps presented the same numbers. The wall-normal velocity fluctuations inside ejections and sweeps were slightly above the complete flow statistics. Ejection events contributed with $20 \%$ and sweep events with $19 \%$ to the total root-mean-square of this velocity component. For low- and high-speed regions, no variation was observed between the conditional and the flow statistics for wall-normal and spanwise turbulent velocity components.

The Reynolds stresses calculated within low-speed structures were remarkably higher than those calculated in the high-speed structures and both higher than those computed for the entire fluid flow. The contribution of the low-speed regions in the total Reynolds stresses was, on average, $37 \%$, while that from the high-speed regions represented 29\%. Similar values were found in the literature. These results lead to the conclusion that the low- and highspeed regions are linked to the turbulent production, which is in line with other researchers observations. 
The average contribution of the ejection regions to the total Reynolds stresses was higher than the sweep regions, being $48 \%$ and $41 \%$, respectively. The higher contribution of ejections in relation to sweeps is in agreement with other studies. These results lead to the conclusion that these structures, which are associated to low- and high-speed regions, are the major contributors to the turbulent production, among the turbulent structures studied in this work.

No difference was found between the statistics computed at vortex structures and the statistics of the global flow regarding the turbulent fluctuations and Reynolds stresses. This fact indicates that the vortices are not directly linked to the turbulent production, although they can induce other events that are related to production, as ejection and sweeps.

Low-speed regions and ejections showed slight overestimation of dissipation rate close to the wall compared to the flow statistics, while high-speed regions and sweep profiles agreed with the overall flow profile. However, the elevated uncertainty of this quantity hampered the quantification of these results, the higher dissipation rate was attributed to the higher probability of vortex existence inside low-momentum regions than inside high-momentum regions, following the literature.

Conditional vortex statistics presented dissipation values remarkably above those of the complete flow statistics. Higher dissipation rates were observed close to the wall, where the most intense vortices were detected. The vortex contribution to the total dissipation rate was, on average, $25 \%$ with a relative structure area of only $10 \%$ of the total flow area.

The fluctuating vorticity components associated to low- and high-speed structures, ejections and sweeps were seen to be under the values of the complete flow statistics, nevertheless the differences were within the estimated values of uncertainty. Contrarily, the fluctuating vorticity components of vortex structures were remarkably above those of the flow values, as expected. The magnitude of the streamwise component of vortex structures was strongly above the others fluctuating vorticity components. The streamwise component contributed, on average, to $25 \%$ to the total root-mean-square of streamwise fluctuating vorticity of the flow, while wall-normal vortex fluctuating vorticity and spanwise vortex fluctuating vorticity were responsible, each one, to about $19 \%$ of the total. This discrepancy seemed to be result of the vortex filtering due to the orientation of the measurement volume.

The fluctuating streamwise vorticity clearly presented a bimodal distribution, with intensity increasing with the distance from the wall. This distribution was attributed to the detected equal frequency of occurrence of clockwise and counter-clockwise vortices. Near the wall, the distributions of fluctuating 
vorticity components were spread out revealing the probable occurrence of all vortex alignments.

From the fluctuating velocity vector, the displacement orientation of turbulent structures was be computed. The probability density functions of projection angle along wall-normal-spanwise plane of low-speed regions started with a bimodal distribution at low wall-normal positions and tended to a single peak distribution with the increasing distance from the wall. The peaks, at about $60^{\circ}$ and $-60^{\circ}$, indicated that the main population of low-speed regions are lifted along these directions. High-speed regions show analogous behaviour, with initial peaks at about $100^{\circ}$ and $-100^{\circ}$, revealing the main tendency of inward motion of these structures along these directions.

For ejection and sweep structures, the probability density functions of the streamwise-wall-normal projection angle displayed high occurrence at directions slightly lower than $180^{\circ}$, at about $160^{\circ}$, showing the prevailing direction of upward displacement of ejections. Correspondent inward direction of sweep structures, with peak value of about $-10^{\circ}$, was obtained. The PDFs of wall-normal-spanwise angles presented bimodal distributions with similar peak values for low- and high-speed regions.

The radii of the streamwise vortices, in average, rise from about 12 to 20 wall units and the standard deviation from 2 to 5 wall units with increasing distance from the wall for the studied range. The increasing of vortex diameters with wall distance suggest a lift up of the vortices. The average vortex centre separation is in the range of 60 to 80 wall units. These results are in agreement with results from literature studies employing different detection techniques. When scaled by the Kolmogorov length, vortex presented constant mean radius of $5.2 \eta$ and standard deviations of $1.2 \eta$, slightly under the literature values

The wall-normal-spanwise plane employed in the present investigation favoured the detection of quasi-streamwise vortices among the variety of prevailing vortical structures. The conditional statistics presented are linked to the detection procedure and the choice of a wall-normal-spanwise-oriented measurement plane. The streamwise-wall-normal and the streamwise-spanwise projection angles presented peaks at $0^{\circ}$ and $180^{\circ}$, while the wall-normalspanwise projection angle displayed an almost uniform distribution. Results of the present work displayed similar population of clock wise and counterclock wise rotating vortices, nevertheless symmetrical hairpin vortices were rare.

Finally, a comparison of the probability density functions of projection and elevation angles with works from the literature were in good agreement. An analysis of the influence of filters, measurement techniques and vortex 
detection methods was conducted. It was demonstrated that the application of a filter to the data changes the most probable inclination and, because of that, must be use with attention.

The qualitative and quantitative results obtained in the present work allow for a discussion of turbulent organization close to the wall. Among the structures studied, vortices appear to play a significant role and are known to be a link with the log layer in agreement with [Carlier and Stanislas, 2005]. Results showed higher occurrence of asymmetrical vortices neighbouring mainly low-speed regions, which is in agreement with observations of [Robinson, 1991b]. The present findings are in line with the turbulent packet proposed by [Schoppa and Hussain, 2000], where an alternating sequence of right and left-handed cane vortices maintains the low-momentum regions. Results are also in agreement with the model proposed by [Adrian et al., 2000b], where low-momentum regions are surrounded by, not only, symmetrical but also asymmetrical hairpins (cane-shaped vortices). The width of low-speed regions is comparable to the distance between the two legs of a hairpin which, in the vicinity of the wall, are considered as quasi-streamwise vortices. The preferred asymmetrical-shaped vortices can be explained according to [Zhou et al., 1999] as a consequence of their faster autogeneration as compared to symmetric hairpins. 


\section{7}

\section{Square Channel Experiments}

The square water channel experiments were performed in a test section constructed in the Fluids Engineering Laboratory at Mechanical Engineering Department of PUC-Rio. The square channel section was previously employed in holographic-PIV experiments in the frame work of a laboratory research line on wall turbulence. Nevertheless, this channel was completely remodelled for the present work to allow for measurements using holographic-PIV, tomo-PIV and hot-wire anemometry. New supports for tomo-PIV camera system and laser was designed and constructed.

This chapter describes in details the remodelled water channel and the tomo-PIV system developed. It is also discussed the optimization process applied to volume illumination, seeding and calibration. The experimental procedures employed to acquire tomo-PIV data are also described.

\subsection{Square Water Channel}

The water channel was $4 \mathrm{~m}$ long with a square cross-section of $40 \times 40 \mathrm{~mm}^{2}$ as illustrated in figure 7.1. The last $1.5 \mathrm{~m}$ of the section was used as a testing zone to allow the flow to be fully developed. The flow developing length upstream of the testing zone was equivalent to 63 hydraulic diameters. The upstream part of the testing zone was reserved for tomographic measurements, while its downstream part to holography. The water was driven from an open reservoir (Alpina 400-litre tank) trough the test section by a Dancor centrifugal pump (model 3OPB56 with $0.75 \mathrm{cv}$ ) and then returned trough a $50-\mathrm{mm}$ pipe to the reservoir to close the circuit. The flow rate was measured by an Ciasey magnetic flow meter (model VMF019 for flow rates between 5.17 and $170 \mathrm{~L} / \mathrm{min}$ with an uncertainty of $1 \%$ ), placed between the pump and the plenum chamber at the channel inlet. Readings from this magnetic flow meter were displayed by an indicator module (Incontrol CEV 1000). The water flow rate through the channel was varied by controlling the rotation of the pump motor by means of a Weg CFW08 frequency inverter. The temperature of the water inside the tank was monitored by a type-K thermocouple (chromel- 


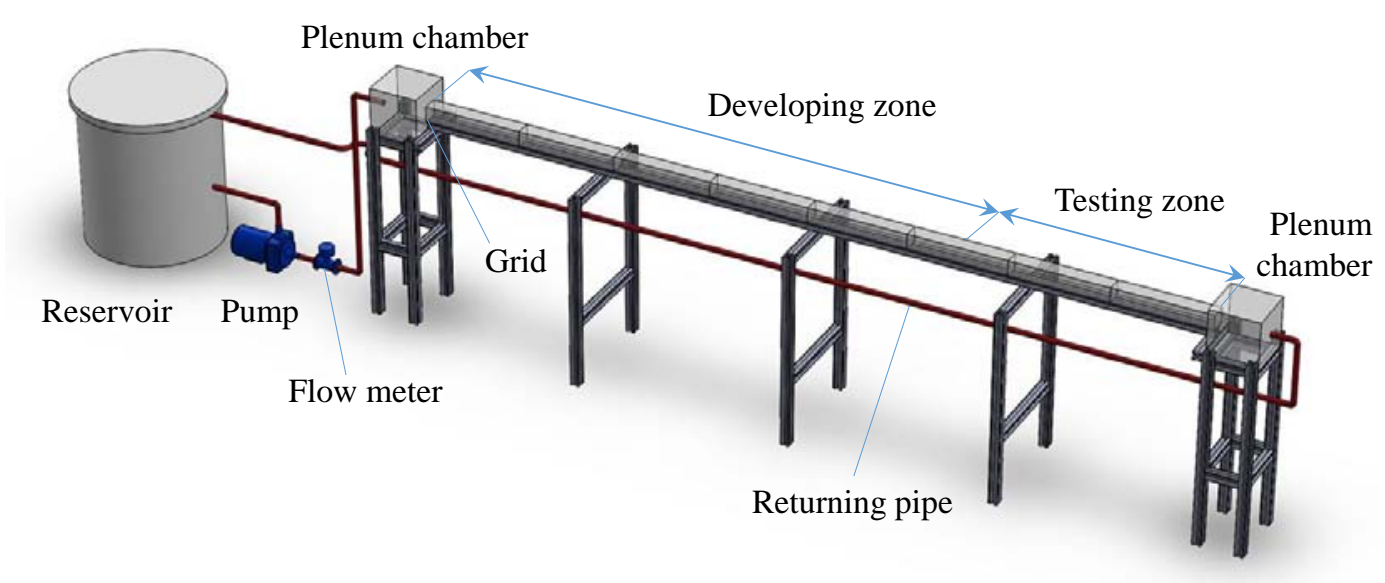

Figure 7.1: Square water channel sketch.

alumel) connected to a Fluke 116 multimeter with $1 \%$ of uncertainty. Valves used to drain the section or to change the flowing circuit were not represented in figure 7.1 for clarity. The water channel was supported by $45 \times 45 \mathrm{~mm}^{2}$ Bosch Rexroth aluminium profiles, which provided a solid structure and facilitated alignment and levelling of the channel section.

The water-channel section was designed for a maximum operating flow rate of $100 \mathrm{~L} / \mathrm{min}$, which corresponds to a turbulent flow with Reynolds number $R e_{D}$, based on the hydraulic diameter, of about 42000. Flow rates above this safe operation value were avoided due to risk of the internal pressure levels damaging the adhesive bonding the Plexiglass channel walls.

Figure 7.2(a) shows a picture of an upstream view of the water channel. The reservoir (bottom-left corner of this image), plenum chamber at the inlet, channel development zone, laser and controlling computer can be clearly seem in this picture. The downstream view of the channel, figure 7.2(b), presents the testing zone with viewing prisms, light optics and cameras.

At the inlet and at the outlet of the test section, $300 \times 300 \times 300 \mathrm{~mm}^{3}$ plenum chambers were added to attenuate oscillations generated by the pump and water passage trough different cross-section shapes (i.e., round pipe to square channel and square channel to round pipe transitions, respectively). Details of the plenum chambers can be observed in figure 7.3. A screen with an orthogonal grid $1 \mathrm{x} 1 \mathrm{~mm}$ was installed at the inlet of the section to accelerate flow transition and to break large-scale vortices coming from the pump and curves. A convergent nozzle was incorporated at the outlet section to minimize pressure fluctuations travelling upstream into the test zone as a result of fluid impact at the end wall of the plenum chamber (figure 7.3(b)).

The channel length of $4 \mathrm{~m}$ was composed by 8, 500-mm-long Plexiglass moduli with a cross-section of $40 \mathrm{x} 40 \mathrm{~mm}^{2}$. Each modulus was carefully 


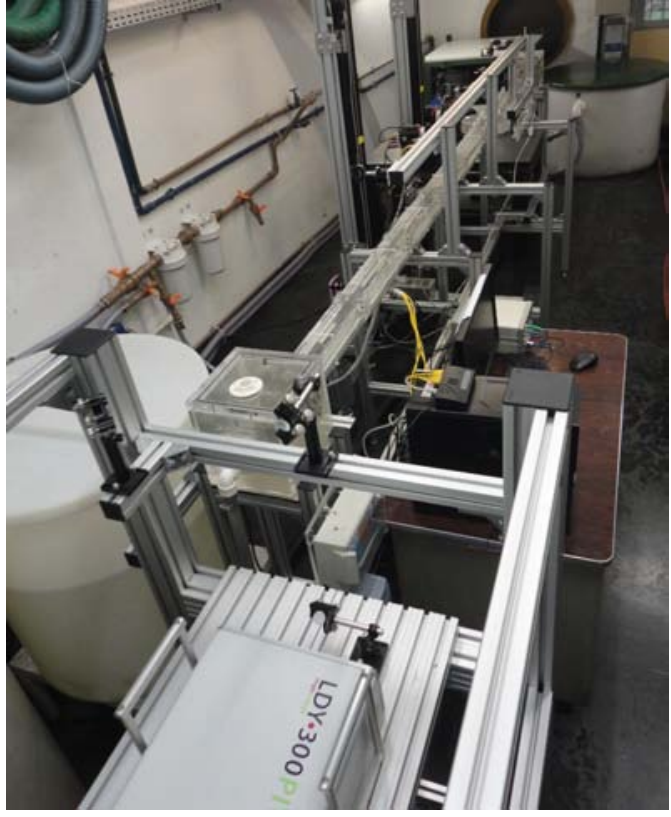

(a)

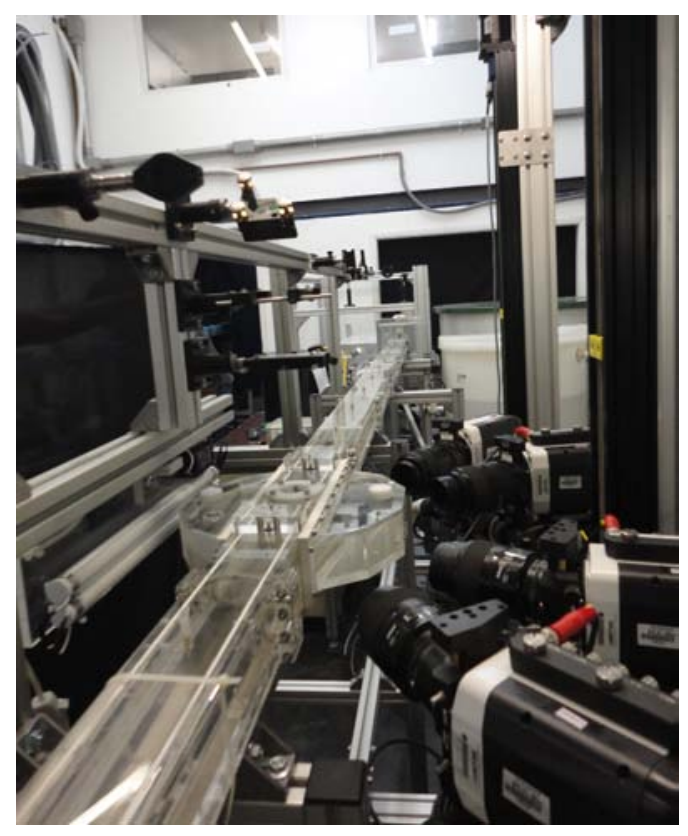

(b)

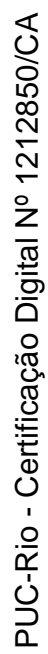

Figure 7.2: (a) Upstream and (b) downstream pictures of the water channel.

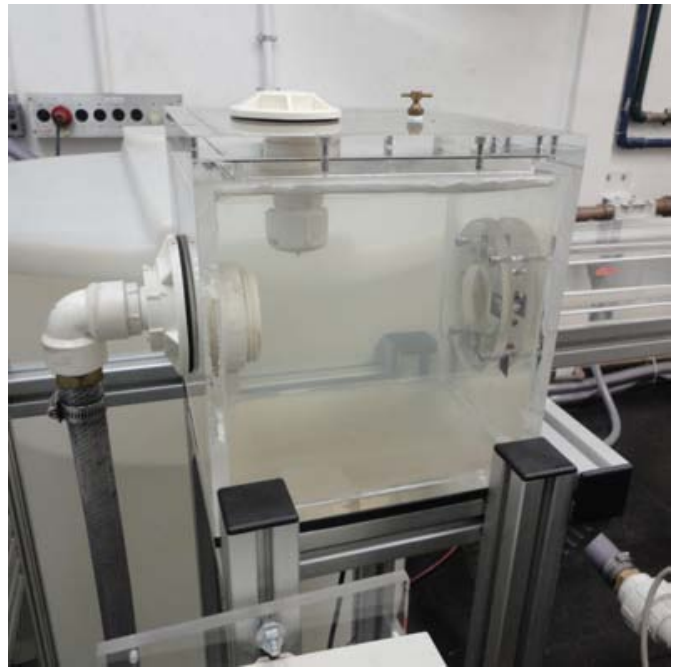

(a)

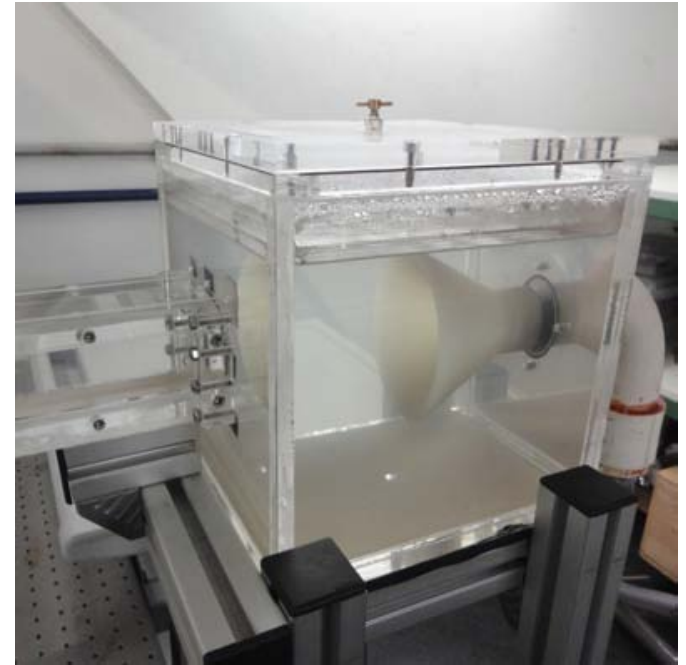

(b)

Figure 7.3: Plenum chambers at (a) inlet and (b) outlet of the water channel. 


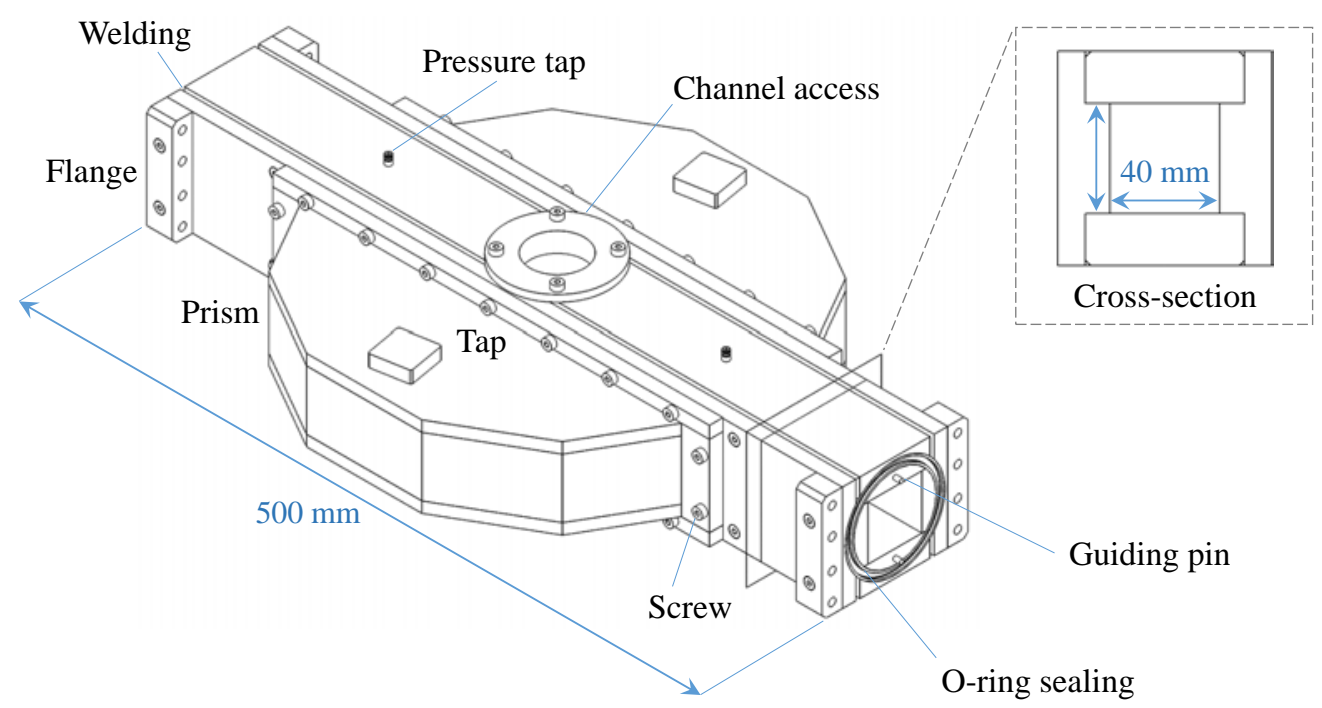

Figure 7.4: Water-channel modulus of the tomo-PIV testing zone showing the detail of the squared cross-section.

constructed by gluing four precisely machined Plexiglass plates to ensure that after assembly the internal dimensions were within tight tolerance levels. The plates were bonded with chloroform to give mechanical rigidity and to withstand the internal water pressure during the flow. To avoid leakage trough assembled parts of the moduli, an external welding with polypropylene was employed in addition to the traditional gluing. The moduli were connected using flanges with guiding pins to ensure proper positioning and the sealing was obtained by rubber o-rings. Pressure taps in the form of 1-mm holes were installed along the test section wall, equally spaced of $250 \mathrm{~mm}$. All moduli of the water channel were similar to the testing zone modulus, which is sketched in figure 7.4. The differences compared to the testing zone modulus were the channel access and the prisms. The channel access was provided to allow for access to calibration targets in the channel. Also, the access port made possible the use of probes for measuring flow properties.

The prisms, filled with water, were added to the modulus at the testing zone in order to attenuate distortions due to different refraction indexes. As common practice in stereo PIV experiments ([Van Doorne and Westerweel, 2007]), when water is used as working fluid, the interface between air and the wall of the channel was oriented normal to the camera viewing directions by adding optical prisms. A secondary prism was installed symmetrical to the first one to enable back illumination of the transparent target employed for the cameras calibration operation. A sample of distortion differences observed by using and not using the prisms is presented in figure 7.5. For the camera arrangement employed (see section 7.2), significant changes were noticed along the width of the target images, while no relevant difference appeared along the 


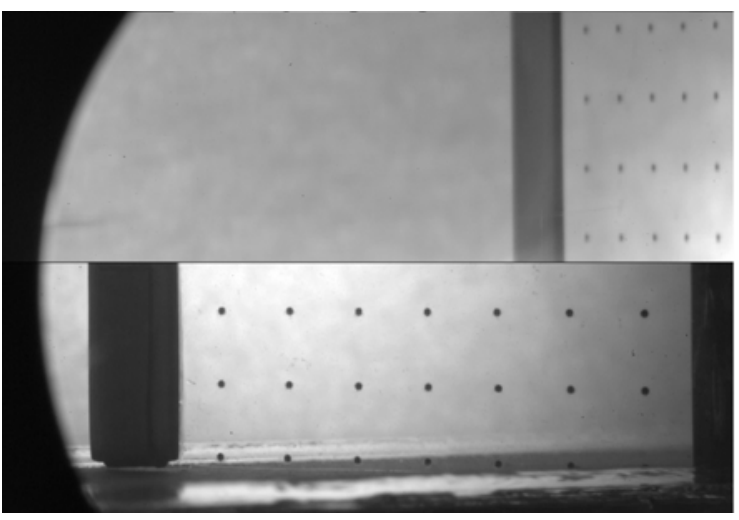

(a)

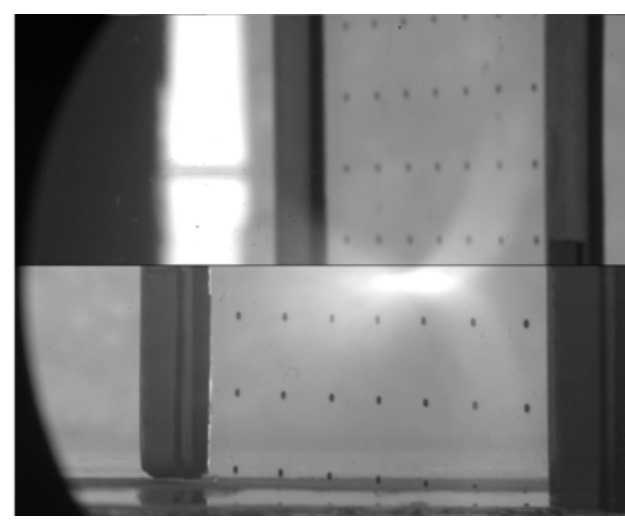

(b)

Figure 7.5: Sample pictures of the camera calibration target imaged by (a) camera 1 and (b) camera 2 employing (bottom) and not employing (upper) prisms, for the same camera configuration.

height, as expected for this configuration. The main discrepancy regarding the prism use came out for camera 1 as a consequence of the smaller viewing angle compared to camera 2. Because of the smaller viewing angle, the light path of camera 1 passes through a longer length inside the wall, resulting in a difference of two times in the magnification along the width in favour of the prism use.

\subsection{Tomo-PIV Setup}

The tomographic-PIV system consisted of four Phantom Miro M340 CMOS cameras $\left(2560 \times 1600\right.$ pixels at $800 \mathrm{fps}$, pixel size of $10 \mathrm{x} 10 \mu \mathrm{m}^{2}$, internal RAM of 12 GB, Mono, 12-bits), in a linear arrangement ([Scarano, 2013]), which were mounted with LaVision Scheimpflug adapters assembled to expansion rings and 105-mm Micro-Nikkor Nikon lenses at $f \# 32$. Figure 7.6 depicts the tomo-PIV setup, while figure 7.7 shows a top view picture of the camera arrangement. Camera parameters and angles can be found in table 7.1. Prisms in front of the cameras have been used to correct the change in refractive index at the channel wall and to attenuate astigmatism as mentioned previously. The tomo-PIV investigation volume was illuminated by a Litron LDY304 laser (2x30 $\mathrm{mJ}$ at $1 \mathrm{kHz})$ in a $2.5-\mathrm{mm}$ thick wall-normal-spanwise sheet. The volume was located at about $2.75 \mathrm{~m}$ from the beginning of the channel section to allow the development of the flow. The laser beam was guided by mirrors to pass trough a Newport plano-convex N-BK7 cylindrical lens with $-75.6 \mathrm{~mm}$ of focal length and then trough knife-edge filters, before entering the test section from the top wall. The total measurement volume was $2.5 \times 22 \times 26 \mathrm{~mm}^{3}$ in the streamwise, wall-normal and spanwise directions, respectively. The position of this investigation volume is displayed in the channel front view in figure 7.6. 
Table 7.1: Camera parameters.

\begin{tabular}{cccccc}
\hline camera & camera type & pixel $(\mu \mathrm{m})$ & lens $(\mathrm{mm})$ & $\mathrm{f} \#$ & $\theta_{y}(\mathrm{deg})$ \\
\hline 1 & Phantom M340 & 10 & 105 & 32 & -30 \\
2 & Phantom M340 & 10 & 105 & 32 & -60 \\
3 & Phantom M340 & 10 & 105 & 32 & -120 \\
4 & Phantom M340 & 10 & 105 & 32 & -150 \\
\hline
\end{tabular}

The laser was plugged in an autotransformer that raised the laboratory voltage from 220 up to $240 \mathrm{~V}$.

The tomo-PIV data acquisition was performed by the Phantom Camera Control software (PCC) synchronized by a TSI laser pulse synchronizer (model 610036 with 8 channels), programmed using the Insight 4G software (also by TSI).

\subsection{Volume Illumination}

The laser beam, after being reflected in guiding mirrors and passing through the diverging lens, was reflected in a $45^{\circ}$ mirror and then passed trough a knife-edge filter mounted along the spanwise direction, followed by a similar filter along the streamwise direction before entering through the top wall of the water channel. Figure 7.8 shows pictures of the light path with optics taken during an experimental test. Figure 7.8(b) presents the last part of the light path. The knife-edge filters shaped the light sheet as a top-hat profile, commonly used for tomo-PIV ([Scarano, 2013]), to the dimensions of $2.5 \times 22 \times 26 \mathrm{~mm}^{3}(x, y, z)$. Larger volume thicknesses were previously tested. For the same particle seeding employed, the increase in the thickness raised the amount of ghost particles deteriorating the quality of tomo-PIV reconstruction. This finding is in agreement with other researchers ([Elsinga et al., 2006, Scarano, 2013, Thomas et al., 2014], to mention just a few).

A different light path entering through the side wall was also tested. Contrarily to what was employed in the present work, the thick wall-normalspanwise sheet was illuminated from the negative spanwise direction (i.e., $z$ axis). The idea was to produce a forward particle illumination and, consequently, profit from the Mie's scattering characteristics (revisited chapter 3). However, the light reflections inside the 19-mm thick Plexiglass wall created a noisy background pattern for cameras 2 and 3, as presented in figure 7.9. This noise deteriorated the particles signal recorded in the images, not allowing for a quality reconstruction. Since the available laser power was sufficient and the 

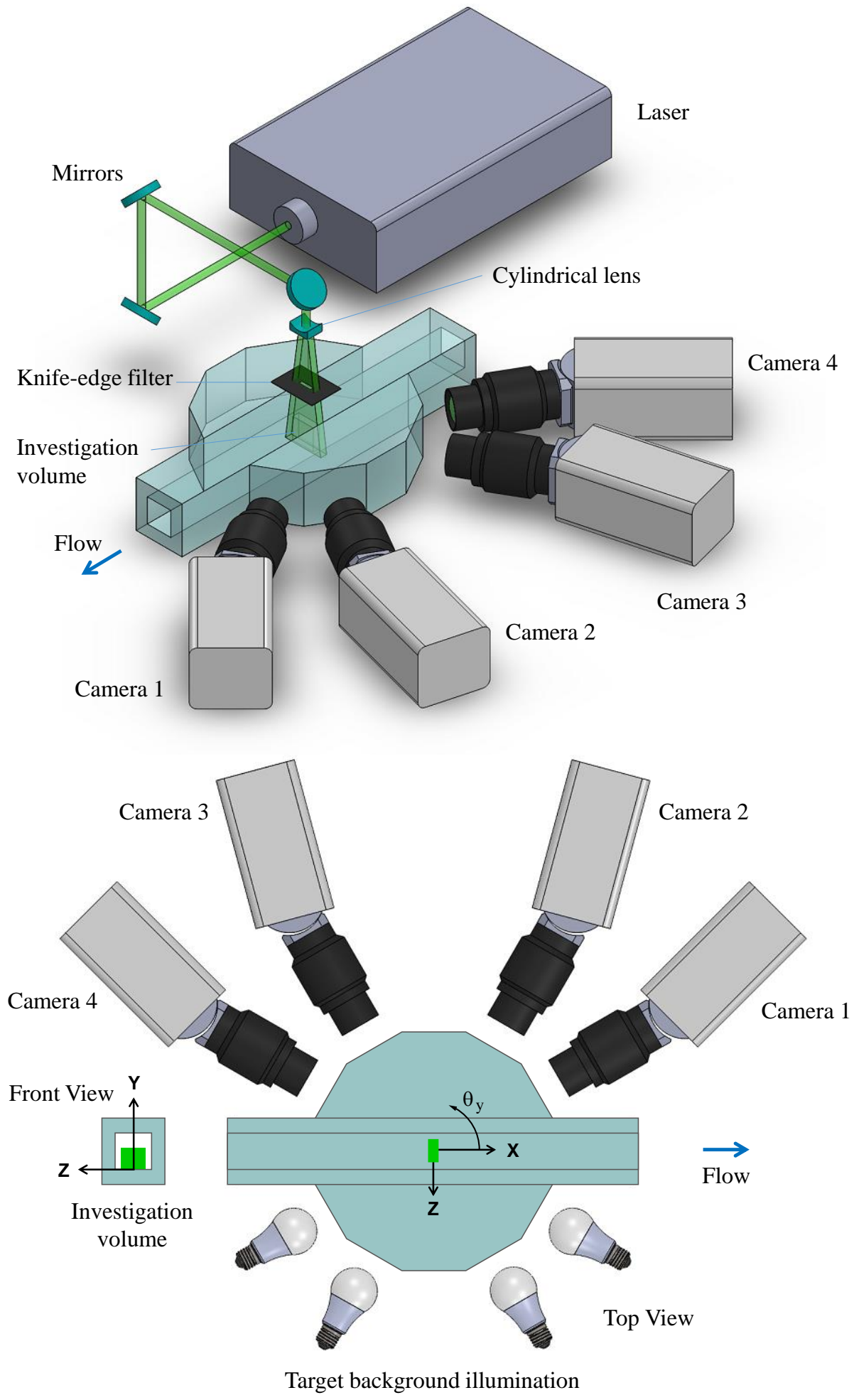

Figure 7.6: Schematic views of camera arrangement in the water-channel experiment. 


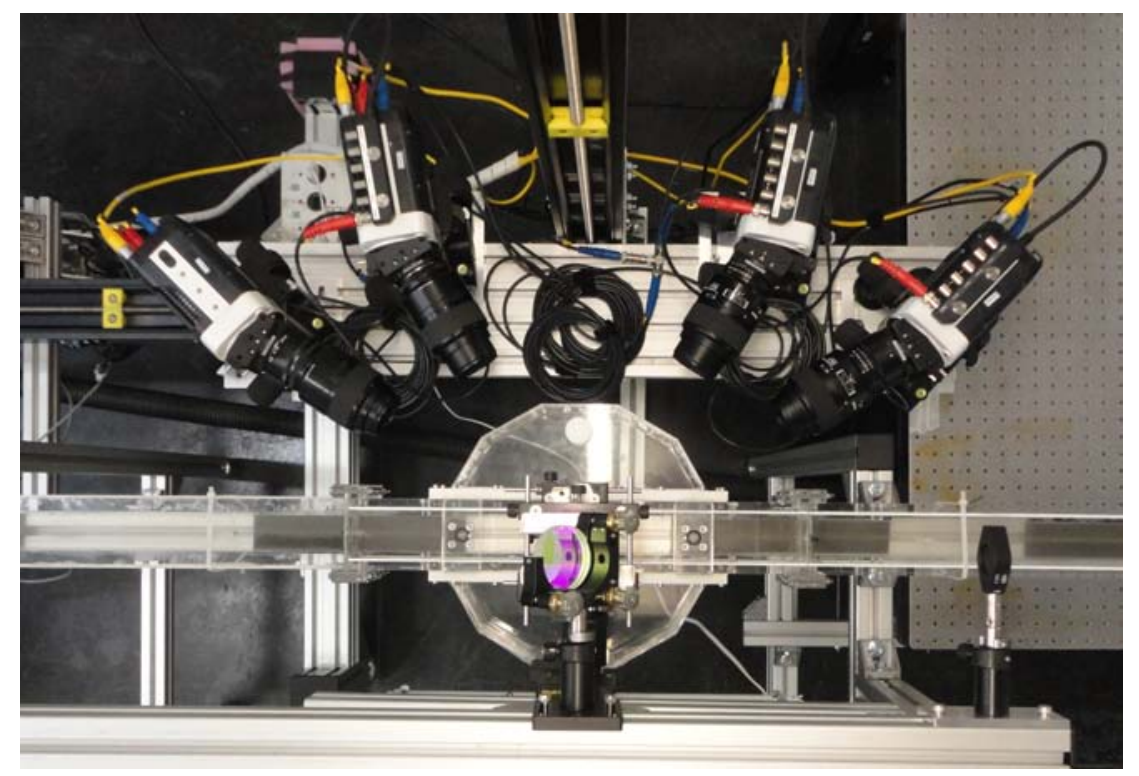

Figure 7.7: Top view picture of the camera arrangement in the water-channel

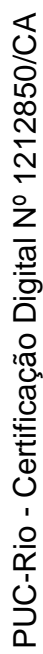
test section showing the Scheimpflug configuration.

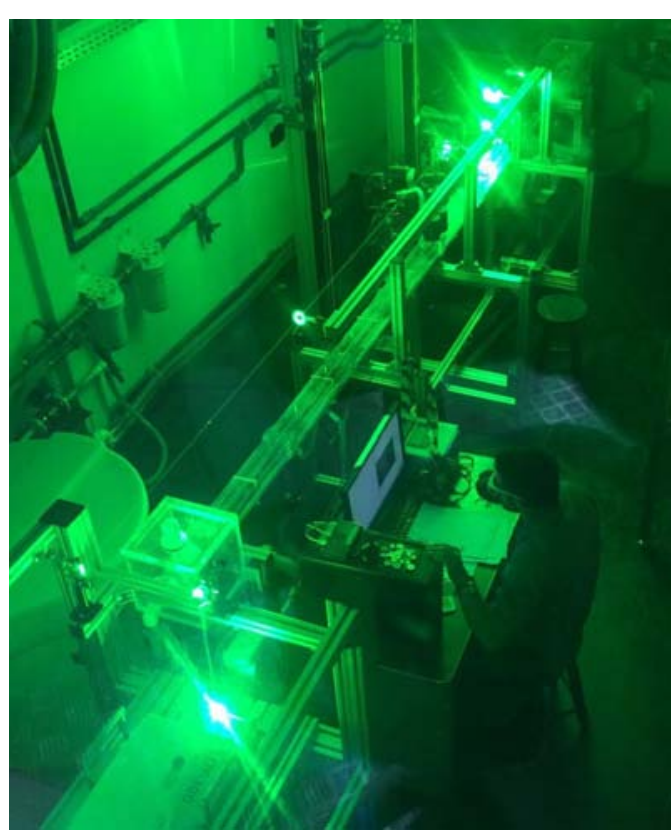

(a)

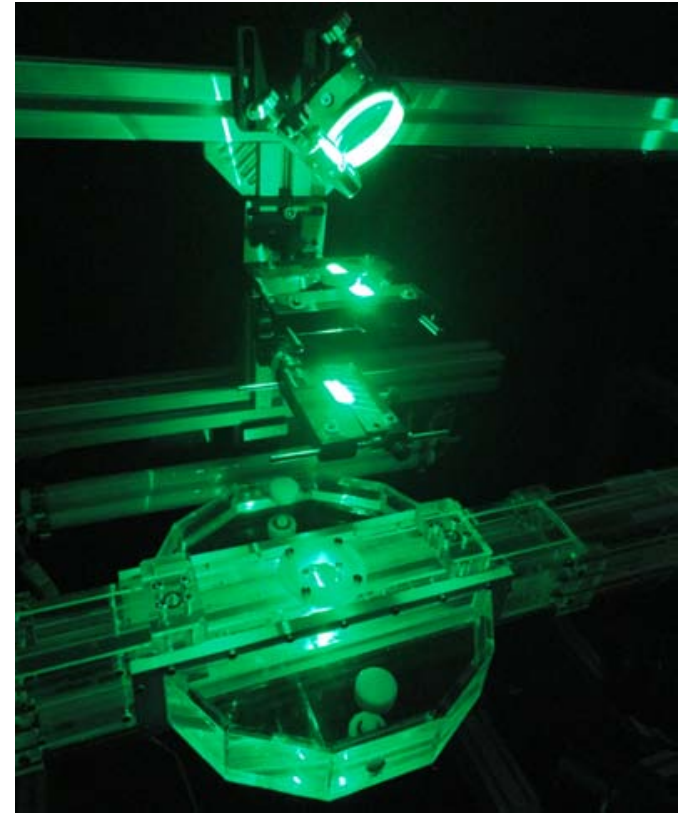

(b)

Figure 7.8: Tomo-PIV experimental acquisition showing (a) upstream and (b) downstream part of the optical path in the water channel. 


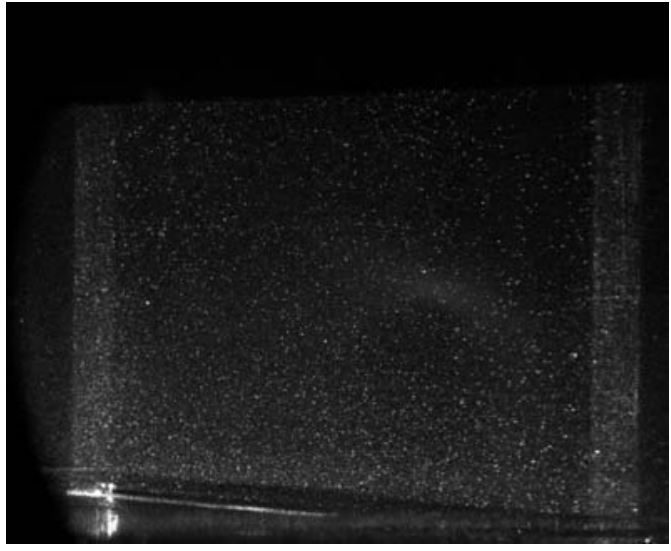

(a)

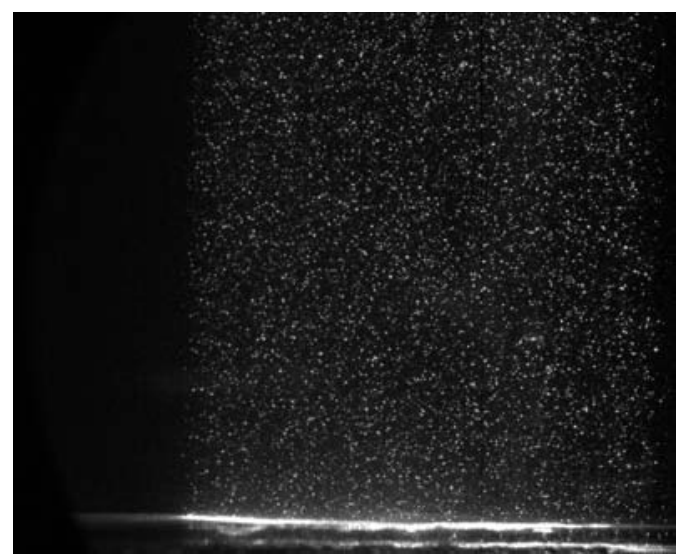

(b)

Figure 7.9: Sample of tomo-PIV particle images recorded by camera 2 employing a laser illumination coming (a) from the side and (b) from the top walls of the water channel.

size of the particles employed in these water experiments was relatively large, the forward scattering illumination scheme was not mandatory, and the illumination was modified to enter the channel through its top wall. The better quality of the particle images obtained with this arrangement can be observed in figure $7.9(\mathrm{~b})$.

\subsection{Seeding}

Tomo-PIV technique requires the addition of tracer particles into the flow. The water flow was seeded with silver-coated hollow glass sphere particles (Potters Industries SH400S20, 20\% of silver in weight, average diameter of $13 \mu \mathrm{m}$, average density of $1.6 \mathrm{~g} / \mathrm{cm}^{3}$ ).

Since the channel wall was thick due to construction constrains (19 mm), the particles presented significant distortion, displaying aberrations and astigmatism for low camera numerical aperture values. In addition, the cameras set with low $f \#$ were not able to focus the entire investigation volume, even employing Scheimpflug adaptors. A numerical aperture of $f \#=22$, as usually used in tomo-PIV water experiments (e.g., [Schanz et al., 2014, Lynch and Scarano, 2015, Schanz et al., 2016]), avoided particle blurring effects due to limited depth of field. However, astigmatism was still presented and pushed the f-number regulation to 32. Similar problem was faced by [Scarano and Poelma, 2009] during a cylinder wake experiment inside a water channel. In that work, the $f \#$ of 22 , sufficient to focus particles inside the illuminated volume, was increased to 32 because of aberrations.

Particles were recorded in the images with diameters between 2 and 8 pixels, which can be verified in figure 7.10 of an amplified particle image 


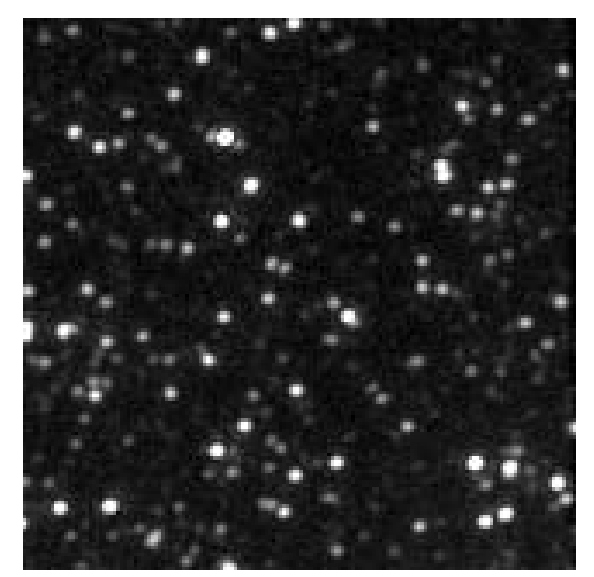

Figure 7.10: Zoom of an area of 160x160 pixels of a particle image sample from camera 2.

from camera 2 . That value agreed with the estimate of the normalized particle diameter in the image, $d_{\tau}^{*}$, of about 6 pixels given by eq. (3.2.2) for the experimental parameter values employed, i.e., $M$ displayed in table $7.3, f \#=$ 32 , and $\lambda=0.527 \mu \mathrm{m}$. This particle diameter was about 3 times larger than the recommended particle size in the literature ([Scarano, 2013]). The possible change to smaller particle tracers would not affect the particle diameter in the images, since it was dominated by particle image diffraction computed by eq. (3.2.3). The particle size could only be changed by the reduction of $f \#$ in this experimental configuration. The problem of particle size was considered to be less significant compared to particle aberrations, described in the previous paragraph. So, the $f \#$ was kept at 32 .

The particle concentration was increased up to a source density of about 0.16, as suggested by [Scarano, 2013] for a 4-camera tomo-PIV system. The initial guess of the mass amount of tracer particles to be added into the flow was calculated from the source density according to eq. (3.2.6). The total mass $m_{\text {total }}$ is a function of the particle concentration.

$$
m_{\text {total }}=C V_{\text {vol }} m_{p}
$$

where $V_{v o l}$ is the water volume inside the channel (260 L for the present work) and $m_{p}$ is the mass of a tracer particle that can be given by

$$
m_{p}=\frac{\rho_{p} \pi}{6} d_{p}^{3}
$$

where, for the tracers used, the average density $\rho_{p}$ was $1.6 \mathrm{~g} / \mathrm{cm}^{3}$ and the average diameter $d_{p}$ was $13 \mu \mathrm{m}$.

The particle concentration in the experiment can be estimated from eq. (3.2.6)

$$
C=\frac{4 N_{s}}{\pi \triangle z}\left[\frac{M}{d_{\tau}}\right]^{2}
$$


where $N_{s}$ is the source density, the illuminated volume thickness $\triangle z$ was $2.5 \mathrm{~mm}$, the magnification $M$ was 0.5 , the averaged image diameter of the particle was estimated previously as 6 pixels and the camera pixel size $\triangle_{\text {pixel }}$ was $10 \mu \mathrm{m}$.

Setting the source density to the recommended value of $N_{s}=0.16$, the estimated total mass of tracer particles to be added in the experiment was $2.7 \mathrm{~g}$. This mass amount was slightly lower than the actual mass added to the flow. The particles were carefully added regarding the calculated source density from camera images. The number of particles per pixel was estimated using Matlab code by dividing the amount of local intensity peaks in particle distribution images by the respective image area of the investigation region. To avoid underestimation of the amount of particles due to their superposition (more critical in cameras 2 and 3 due to the bigger length of the LOS crossing the illuminated volume than cameras 1 and 4), the knife-edge filter along the streamwise direction was adjusted to shape the volume to half of its normal thickness (as proposed by [Lynch and Scarano, 2014]). So, the particle concentration of the experimental run was indirectly calculate by doubling the particle concentration value of this thinner volume. Table 7.2 shows the average amount of particles, particle density and source density for the analysed experimental run, indirectly calculated by this in-house developed code. The total final mass of tracer particles was composed by the sum of particle mass inserted in the flow during the particle loading process, up to the amount displayed in table 7.2. The particle mass was measured employing an analytical balance with automatic calibration (Gehaka, model AG200, for mass measurements between 0.01 and $199.9990 \mathrm{~g}$ with a repeatability of $\pm 0.0002 \mathrm{~g}$ ). The total amount of particle tracers added was $3.5 \mathrm{~g}$. A possibly explanation to the need of this extra mass of particles can be linked to the fact that particles could get trapped in pipe connections, valves and pump, retained on channel surfaces and settled with time. The latter fact was the severest one, since the average density of the particles used was $1.6 \mathrm{~g} / \mathrm{cm}^{3}$. Some measures were adopted to mitigate the referred problem, such as recirculating water time to time, even when the test section was not being used to acquire data. In addition, the water inside the reservoir was constantly mixed with a stick, but the same procedure was not possible in the plenum chambers. Please revisit figure 7.3 to have an idea of the amount of particles settled on the bottom part of the plenum chambers after some days.

Figure 7.11 shows samples of particle images of the water-channel experiment recorded by camera 3. Cameras 2 and 3 experienced larger particle densities $\left(p p p\right.$ and $N_{s}$ ) than others for the same amount of particles in the 
Table 7.2: Average amount of particles in each camera for the water-channel tomo-PIV experiment.

\begin{tabular}{cccc}
\hline camera & number of particles & particle density $(p p p)$ & source density $\left(N_{s}\right)$ \\
\hline 1 & 18543 & 0.008 & 0.157 \\
2 & 18439 & 0.009 & 0.176 \\
3 & 18792 & 0.010 & 0.196 \\
4 & 18594 & 0.008 & 0.157 \\
\hline
\end{tabular}

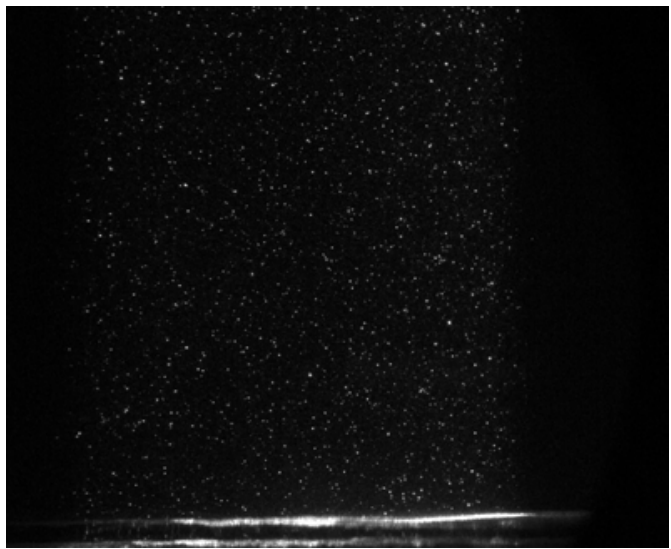

(a)

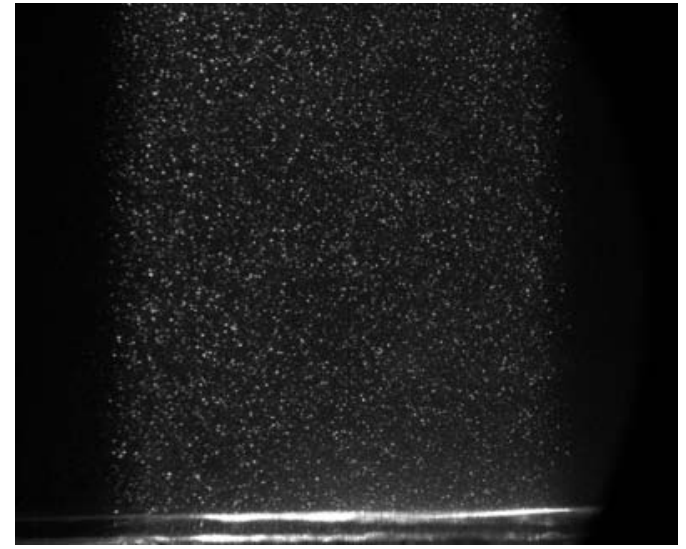

(b)

Figure 7.11: Sample of particle images of the water-channel tomo-PIV experiment recorded by camera 3. Particle concentration of (a) $0.004 \operatorname{ppp}\left(N_{s}=\right.$ $0.078)$ and (b) $0.010 p p p\left(N_{s}=0.196\right)$.

flow due to these camera viewing angles. The images of low particle concentration were used to improve the calibration (section 7.6), while images with about 0.010 ppp were used in the tomo-PIV processing of the present work (section 8.1).

The 12-bit particle images acquired displayed a gray level distribution, not including reflections at the wall, from 0 to 4095. The summary of image parameters is exposed in table 7.3, where cal is the conversion between image and laboratory dimensions, calculated by the calibration-target images, and the magnification is the ratio between imaged and real objects computed by $M=$ cal $\triangle_{\text {pixel }}$. The subscripts $y$ and $x$ refer to the vertical and transverse image directions, respectively (analogous to coordinates of figure 4.8).

\subsection{Experimental Procedure}

The following experimental procedures were adopted in order to produce reliable tomo-PIV results and to avoid accidents.

1. Checking valves opening in the pipe circuit of the water channel. As mentioned previously, although these valves were not shown in the 
Table 7.3: Image parameters.

\begin{tabular}{ccccccc}
\hline camera & bits & image (pixels) & $M_{y}$ & $c a l_{y}(\mathrm{pixel} / \mathrm{mm})$ & $M_{x}$ & $c a l_{x}(\mathrm{pixel} / \mathrm{mm})$ \\
\hline 1 & 12 & $2112 \times 1440$ & 0.55 & 54.7 & 0.51 & 51.1 \\
2 & 12 & $1856 \times 1520$ & 0.62 & 61.5 & 0.36 & 36.0 \\
3 & 12 & $1856 \times 1520$ & 0.63 & 63.1 & 0.37 & 37.2 \\
4 & 12 & $2112 \times 1440$ & 0.56 & 56.0 & 0.52 & 51.8 \\
\hline
\end{tabular}

schematic figure 7.1, they were present to keep the channel filled with water even with pump off, to regulate the pressure inside the channel and in the pump outlet during the flow, to change the flowing circuit and to drain the water from the reservoir.

2. Recirculating the water inside the channel (with pump on) for about 4 hours in order to avoid the operational transient regime of the water channel.

3. Mixing the water inside the reservoir with a stick to reinsert the particles retained on the walls and settled into the water. If tracer particles need to be added, they should be diluted in a beaker with filtered water and shed in the water tank, mixing with a stick during the operation.

4. Starting the acquisition system (composed by cameras, laser, synchronizer and computer). After the achievement of the laser temperature operation, the laser power was gradually increased to the desired power level in order to warm up the laser head. The light alignment was also checked in this experimental procedure step employing a low laser power level.

5. Acquisition of preliminary images to check the communication and synchronization of the overall system. The synchronization was done by a laser pulse synchronizer driven by the Insight $4 \mathrm{G}$ software. The images were acquired by means of Phantom Camera Control software (PCC). It is important to mention that the CMOS cameras employed should have a background reference to reduce noise. This procedure was performed by the Current Session Reference (CSR) command in the PCC software with cameras equipped with lens covers or mechanical shutters. The use of safety goggles against laser light are mandatory from this point.

6. Acquisition of tomo-PIV images. The system was triggered by Insight $4 \mathrm{G}$ taking advantage of the command software trigger that allowed the discard of first laser pulses. The camera images were saved from the PCC software with a proper name of the experimental run. 


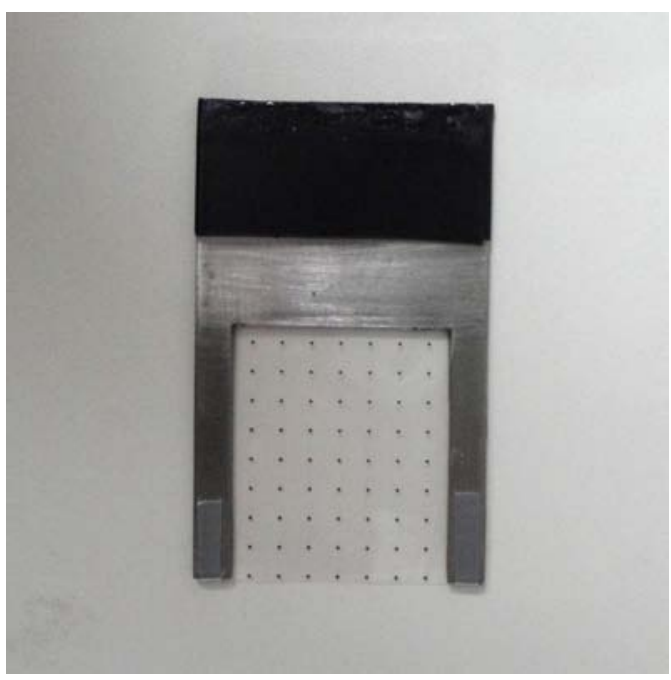

(a)

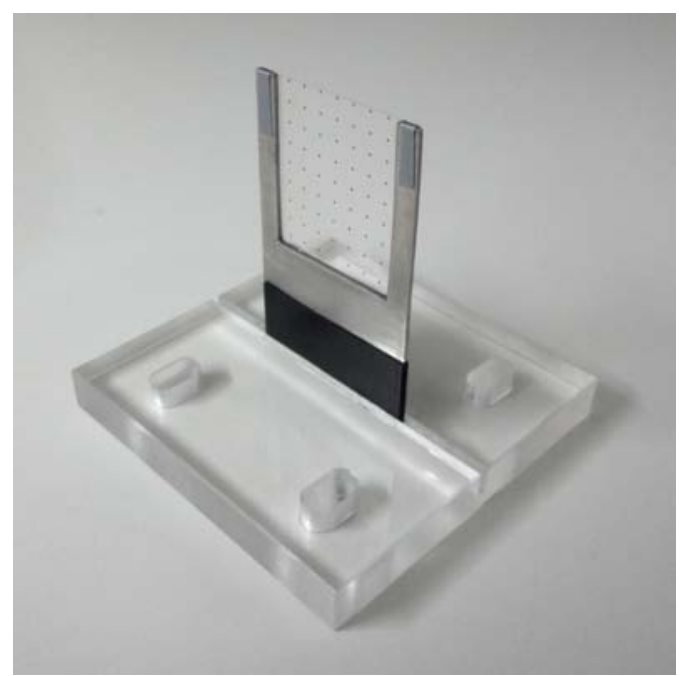

(b)

Figure 7.12: (a) Detail of the transparent target of dot pattern fixed on the stainless-steel frame. (b) Transparent target on its support.

For the present work, the tank was filled with $260 \mathrm{~L}$ of filtered water and the flow rate was fixed to $40 \mathrm{~L} / \mathrm{min}$ (i.e., $R e_{D}=16666$ ). The acquisition frequency was constant at $400 \mathrm{~Hz}$ and the delay between laser pulses that straddled the image frames was set to $400 \mu \mathrm{s}$ in a standard PIV timing configuration ([Raffel et al., 2007]).

\subsection{Calibration}

In the water-channel experiment, the calibration of the cameras imaging the investigation volume was done by means of a specially manufactured 2D target placed inside the water channel at the wall-normal-spanwise orientation. The calibration target was composed by a transparent, 0.2-mm-thick plastic sheet with a dot pattern printed on it. The plastic sheet was fixed on a U-shaped stainless-steel frame with dimensions of $1.5 \times 64.8 \times 39.5 \mathrm{~mm}^{3}$ corresponding to the streamwise, spanwise and wall-normal axis. A pattern of 10x10 dots with diameters of $0.4 \mathrm{~mm}$ distributed on a grid with $4.0 \mathrm{~mm}$ of space was printed. Since the transparent plastic sheet of 0.2-mm thickness was used, the printed dots could be visualized from both sides without significant refraction problems as the ones reported in the wind-tunnel experiment (chapter 4). Figure 7.12 shows the transparent target.

During the calibration procedure, instead of employing the usual method of moving the calibration target along the streamwise direction, the cameras were displaced. Note that displacing cameras to one side was equivalent, for calibration purposes, to displace the target at the same distance in the opposite direction. The cameras were fixed on Manfrotto 405 geared heads mounted on 


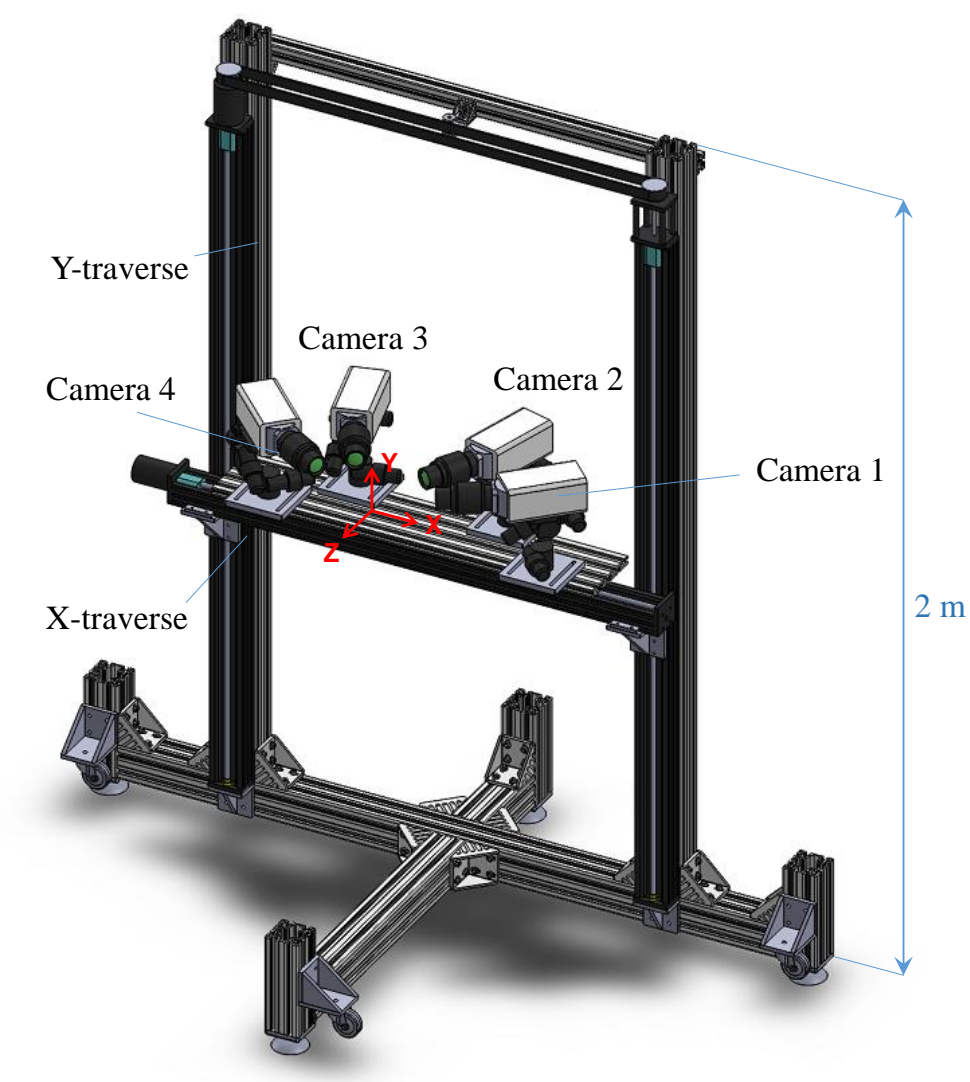

Figure 7.13: Schematic isometric view of the transverse structure supporting the cameras in the water-channel experiment.

a Bosch Rexroth table fixed to a two-axis Velmex controlled traverse. The cameras were disposed in a linear arrangement (or single-plane arrangement) proper for translation purposes during the calibration. The tomo-PIV system allowed cameras to displace of about $1.7 \mathrm{~m}$ along the streamwise direction with $2.5 \mu \mathrm{m}$ of precision and about $1.7 \mathrm{~m}$ along the wall-normal with $5 \mu \mathrm{m}$ of precision. The system was supported by a rigid 90x90 mm² Bosch Rexroth structure. Figure 7.13 presents the transverse structure of the cameras.

Placing the calibration target inside the water channel at the measurement region would be a complex operation since it would be necessary to disconnect the channel moduli or to open the outlet plenum chamber, not mentioning the fact that the reconnection could change the measured positions. To overcome this possible problems, the test modulus had a channel access in the middle to facilitate placement and fixation of the calibration target (please revisit figure 7.4).

For the calibration procedure, initially, the target, placed inside the test modulus, was aligned with the laser sheet at $z^{*}=0$. The camera table was translated in the out-of-plane direction from $z^{*}=-5 \mathrm{~mm}$ up to $z^{*}=5 \mathrm{~mm}$ with steps of $2.5 \mathrm{~mm}$. At each position, the target projection image on the 
cameras was recorded. The target was illuminated from the opposite side of the cameras by an additional light source. As mentioned before, a prism, identical to the prism used to reduce image distortions, was fixed between light source and channel section and filled with water. This was necessary to produce an homogeneous target background illumination. Figure 7.14 shows the acquisition procedure of calibration target images just described, while figure 7.15 presents the calibration images at $z^{*}=0$ recorded by the four cameras.

The markers of the calibration target, in that case black dots pattern on a light background, were automatically located at 5 target-plane positions by a centroid method for all the cameras. With these point locations, it was possible to produce a mapping of world points onto the camera images. The world coordinate system was chosen far away from the borders of the image, avoiding possible aberrations.

Due to the distortions caused by the refraction index change explained formerly, the Soloff model given by eq. (3.2.10) was employed to calibrate the cameras. This model is a higher order model than the pinhole, used previously for the boundary-layer experiments. Since [Elsinga et al., 2006] do not suggest a tomo-PIV volume reconstruction for triangulation errors greater than 0.4 pixels, which were the case for these initial Soloff mapping functions, an expected self-calibration correction was performed (section 3.2.4).

In order to increase the signal to noise on the disparity maps, particle images of the low-concentration experimental run, with a seeding density of $0.004 \mathrm{ppp}\left(N_{s}=0.078\right)$, were used for the initial self-calibration steps. The self-calibration procedure was computed using a interrogation volume size of $1.1 \times 7 \times 7 \mathrm{~mm}$ (i.e., $x, y, z$ of $37 \times 233 \times 233$ voxels) with $50 \%$ of overleaping in a reconstruction volume of size $82 \times 735 \times 868$ voxels (which gave a disparity maps of the size $3 \times 5 \times 6$ ). The input parameters of the software were: a research region of 7 pixels, a precision in the disparity map of 0.5 pixel, a blob of 5-pixel size and a maximum number of 8000 particles in 40 particle images. As pre-processing, the images were filtered by a history-minimum-background subtraction and an intensity threshold between 0.002 and 0.008 according to the camera image. The outcome 3D-2C disparity vector field was used to correct the camera model. With these new mapping functions of each camera a new self-calibration could be performed. At the total, 5 iterations of the procedure were carried out. The disparity map of camera 1 in the middle of the laser sheet for the first iteration of the self-calibration method is shown in figure 7.16. The disparity maps in the first upper-left corner of the first self-calibration iteration for the 4 cameras are amplified in figure 7.17. 


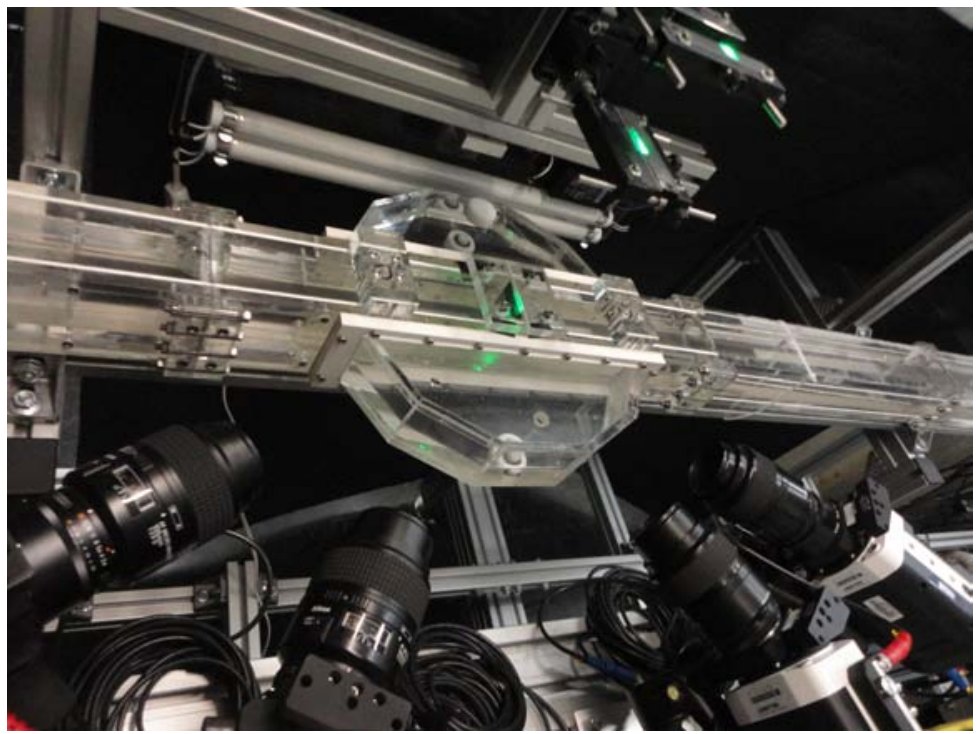

(a)

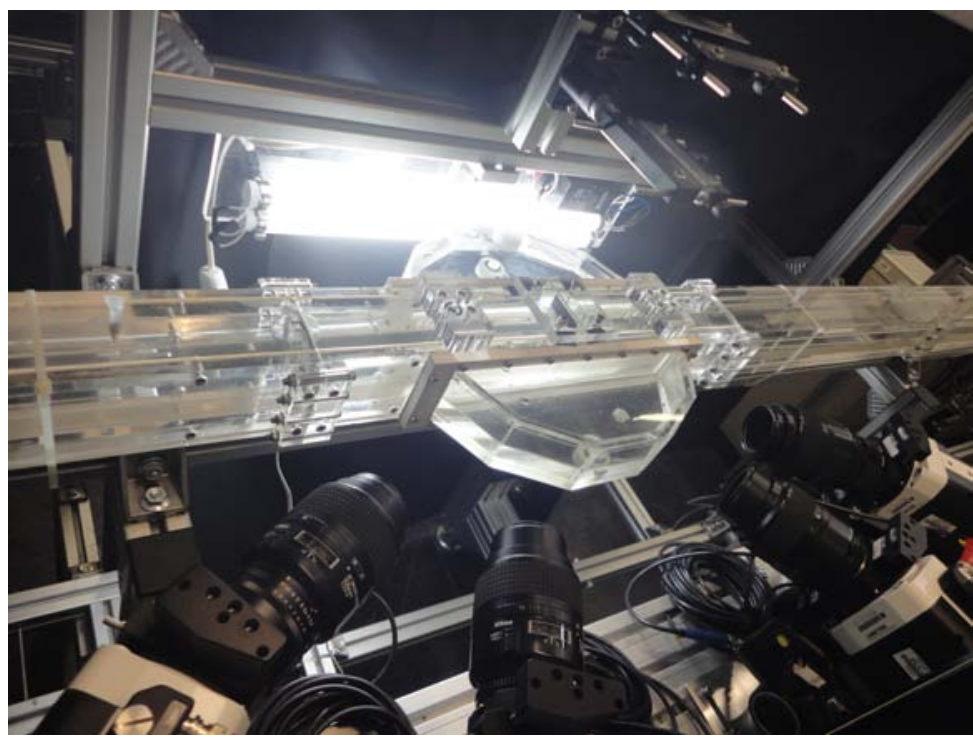

(b)

Figure 7.14: (a) Target alignment with the laser fat sheet at $z^{*}=0$. (b) Recording procedure of target images with background illumination. 

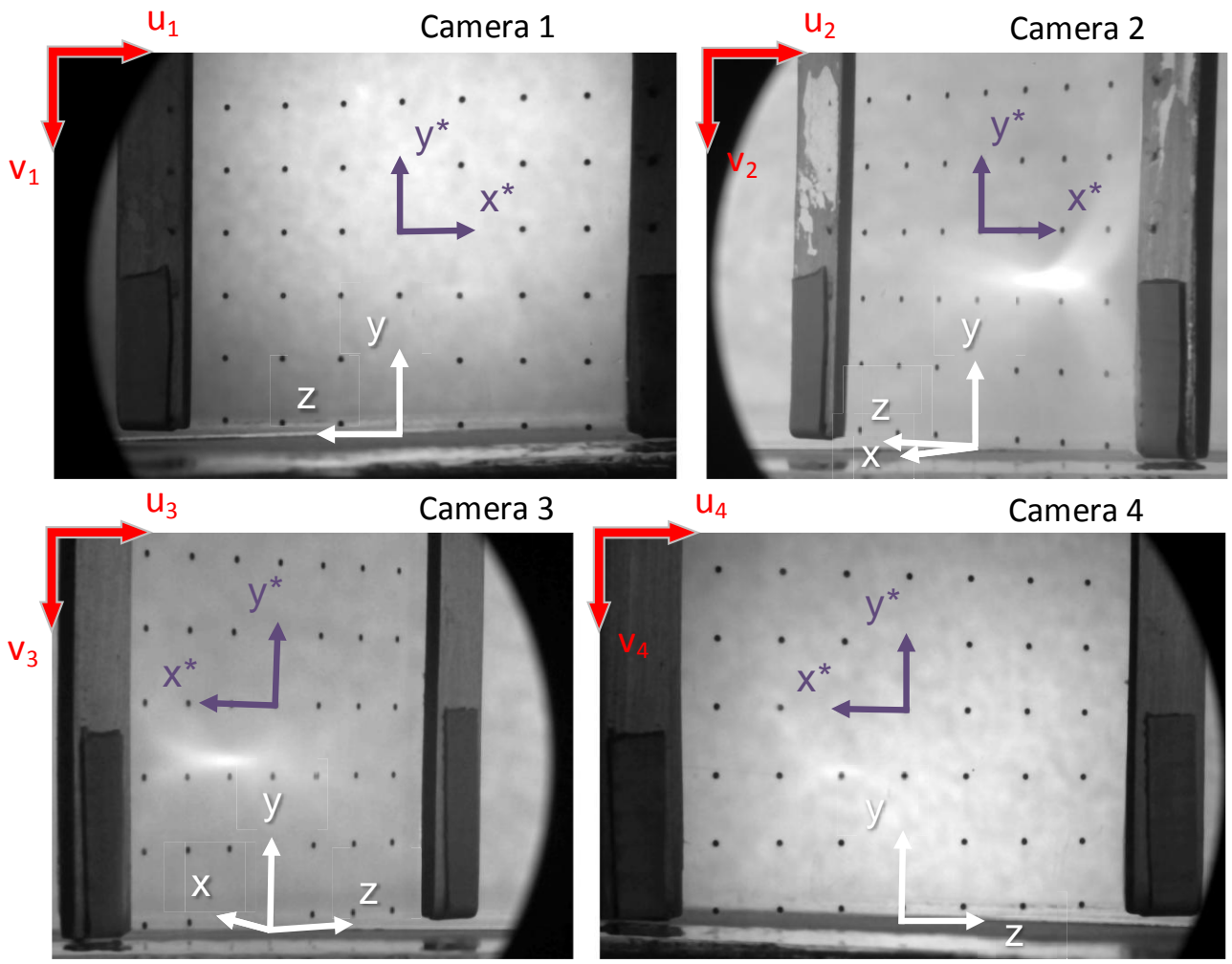

Figure 7.15: Images of the target placed inside the channel at $z^{*}=0$ position for each camera. The images show the image coordinate system (in red), the world coordinate system (in purple) and the channel coordinate system (in white). In order to enhance the visualization the third axis from the world and channel systems can be suppressed sometimes.

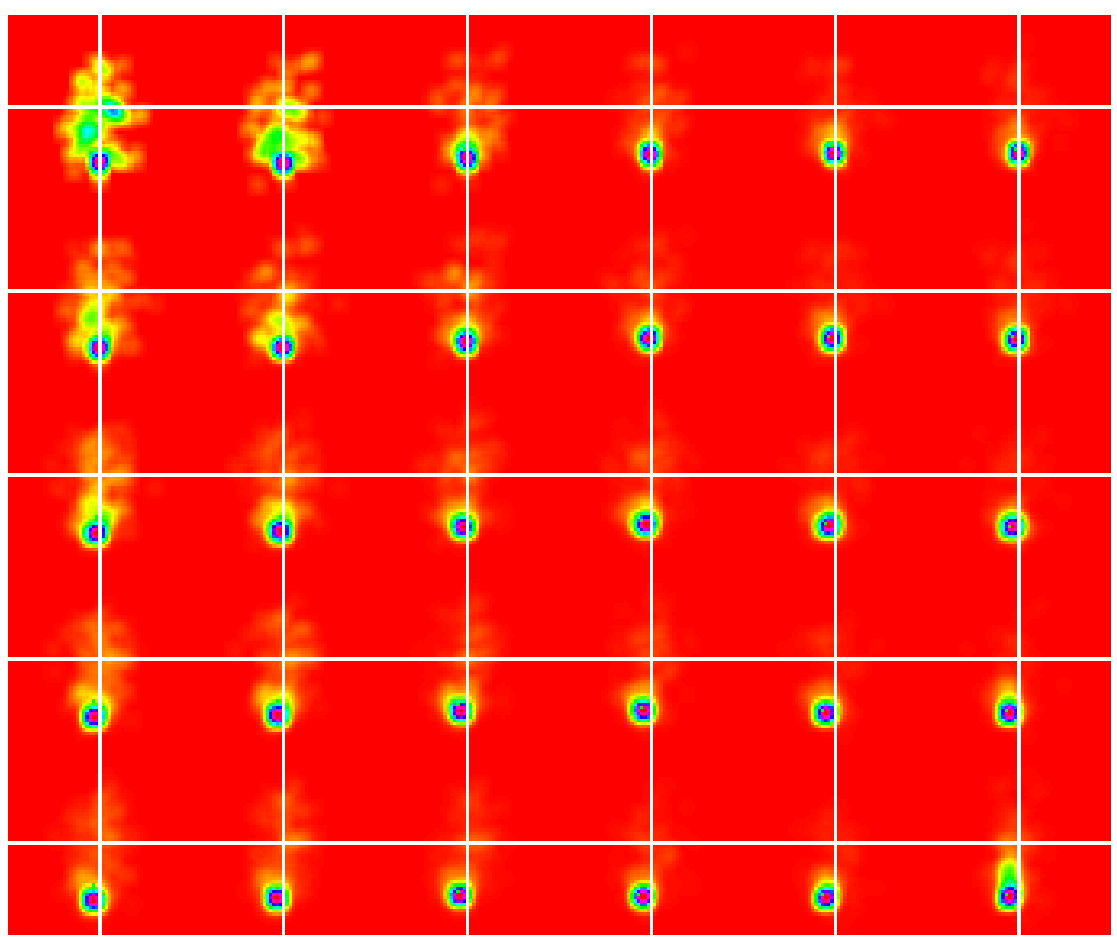

Figure 7.16: 2D disparity map of camera 1 in the middle of the laser sheet for self-calibration method with a search radius of 7 pixels. 
Camera 1

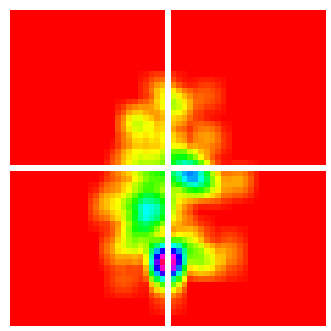

Camera 2

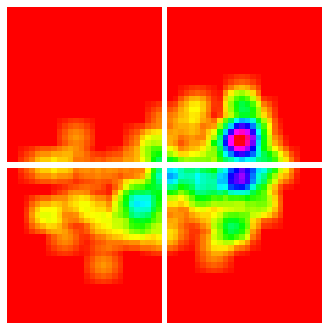

Camera 3

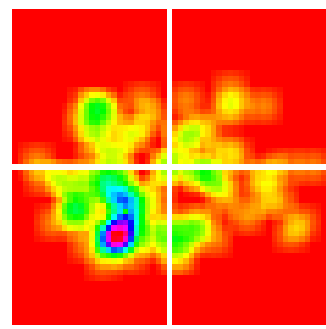

Camera 4

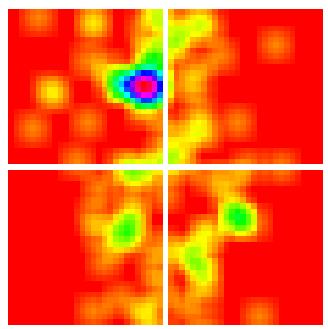

Figure 7.17: Amplified upper-left region of the middle-plane disparity maps for search radios of 7 pixel before self-calibration correction.

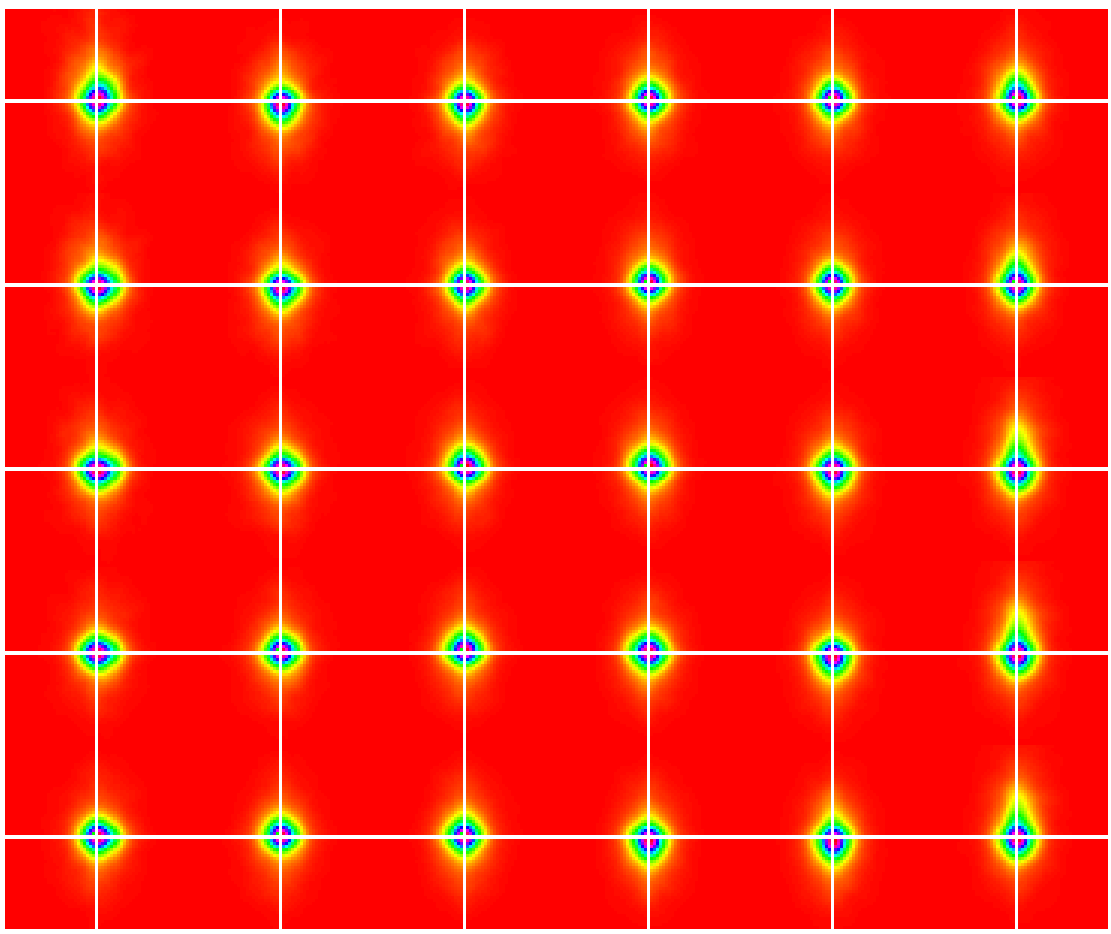

Figure 7.18: 2D disparity map of camera 1 in the middle of the laser sheet for self-calibration method with a search radius of 3 pixels.

The self-calibration procedure was reiterated 5 more times for the experimental images (particle concentration of about $0.010 \mathrm{ppp}$ and $N_{s}=0.196$ ) reducing gradually the research region up to 3 pixels with 0.1 pixel of precision. Applying the self-calibration directly to the high particle concentration run, using a threshold to decrease the amount of particles, did not produce good results. Figure 7.18 presents the disparity map of camera 1 in the middle of the laser sheet after the self-calibration correction. The upper-left corner of the disparity maps after self-calibration correction for the 4 cameras are magnified in figure 7.19 .

After the self-calibration procedure explained before, the errors were significantly reduced as shown in the disparity maps of figures 7.18 and 7.19. The correction of the mapping function by the self-calibration method 
Camera 1

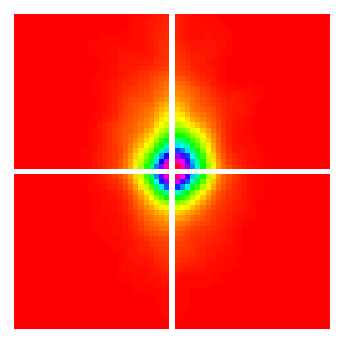

Camera 2

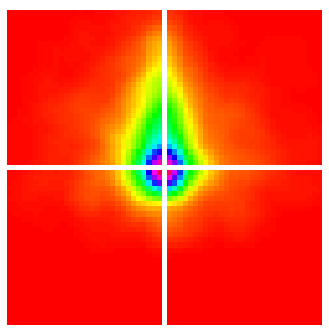

Camera 3

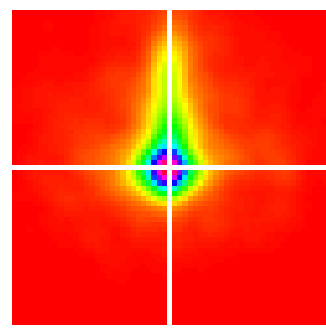

Camera 4

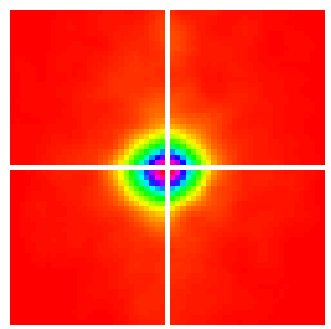

Figure 7.19: Amplified upper-left region of the middle-plane disparity maps for search radios of 3 pixel after self-calibration correction.

Table 7.4: Projection and triangulation errors of each camera before and after self-calibration (in pixel).

\begin{tabular}{cccccccc}
\hline & \multicolumn{3}{c}{ projection error } & & \multicolumn{3}{c}{ triangulation error } \\
\cline { 2 - 3 } & $x_{\text {rms }}$ & $y_{\text {rms }}$ & $\max$ & & $x_{\text {rms }}$ & $y_{\text {rms }}$ & max \\
\hline before & & & & & & & \\
camera 1 & 0.66 & 0.40 & 2.19 & & 3.05 & 0.71 & 4.50 \\
camera 2 & 1.16 & 0.41 & 3.23 & & 1.51 & 2.77 & 4.12 \\
camera 3 & 1.19 & 0.43 & 3.36 & & 1.52 & 2.27 & 5.38 \\
camera 4 & 0.59 & 0.42 & 1.72 & & 3.24 & 1.73 & 6.50 \\
\hline after & & & & & & \\
camera 1 & 0.01 & 0.00 & 0.03 & & 0.05 & 0.05 & 0.10 \\
camera 2 & 0.01 & 0.00 & 0.04 & & 0.07 & 0.05 & 0.20 \\
camera 3 & 0.01 & 0.00 & 0.04 & & 0.04 & 0.05 & 0.15 \\
camera 4 & 0.01 & 0.00 & 0.02 & & 0.04 & 0.05 & 0.15 \\
\hline
\end{tabular}

can be visualized from the decreasing of the errors in table 7.4. The initial Soloff mapping functions had maximum projection errors of about 2 pixels for cameras 1 and 4 and errors of about 3 pixels for other cameras. Cameras 2 and 3 faced a worse calibration than cameras 1 and 4 due to their viewing angles that decreased the magnification along $x^{*}$ direction. The maximum triangulation errors employing the initial mapping functions were 6.5 pixels not allowing a reliable tomo-PIV reconstruction. After the self-calibration, the final Soloff mapping functions had projection errors of about 0.01 pixel and maximum triangulation errors lower than 0.20 pixel in the entire domain, which is enough to produce quality reconstruction volumes ([Wieneke, 2008]). 


\section{8 \\ Square Channel Results}

This chapter presents instantaneous and preliminary statistical results obtained from the tomo-PIV processing of the square water channel flow experiment. The tomo-PIV processing and the optimization applied on the image pre-processing, volume reconstruction and cross-correlation are also discussed.

\subsection{Tomo-PIV Processing}

Tomo-PIV technique was used in the channel flow experiments to extract time-resolved 3D velocity fields employing the same parallelized tomo-PIV software used for the boundary-layer results. It was developed together in a cooperation among the laboratories Pprime, Coria and LML.

An optimization, analogous to that of the boundary-layer experiments (chapter 5), was performed on the water channel data. For the channel data, pre-processing the particle images by minimum-history background subtraction followed by an intensity threshold between 0.002 and 0.008 (according to each camera) and then by a $3 \times 3$ pixels Gaussian filter, was the best option in this case. The intensity threshold was added in the pre-processing procedure due to the background noise generated by the CMOS cameras employed. Figure 8.1 shows a sample of the pre-processing results applied on a particle image from camera 1. It is possible to observe by this image that the background noise and light reflections were sensibly reduced.

The pre-processed images were reconstructed using 8 iterations of BIMART algorithm with MinLOS initialization, a relaxation parameter of 0.2 and a block size equal to four pixels. A 2-iteration volume-filter threshold of 0.001 was employed to reduce the amount of ghost particles. This volume threshold filter should be used with attention. Rising the threshold value increased the signal-to-noise ratio (section 3.3) reducing ghost particles. Although, it had a collateral effect of decreasing slightly the amount of actual particles, which deteriorated the spacial resolution of the resulting velocity field. The reconstructed volume was produced employing a pixel to voxel ra- 


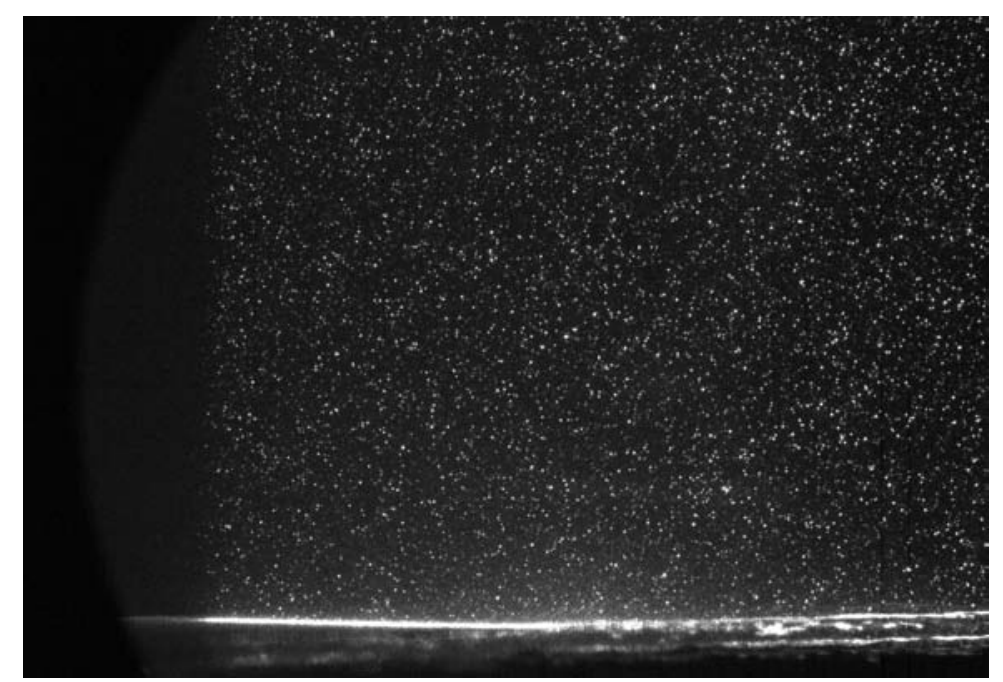

(a)

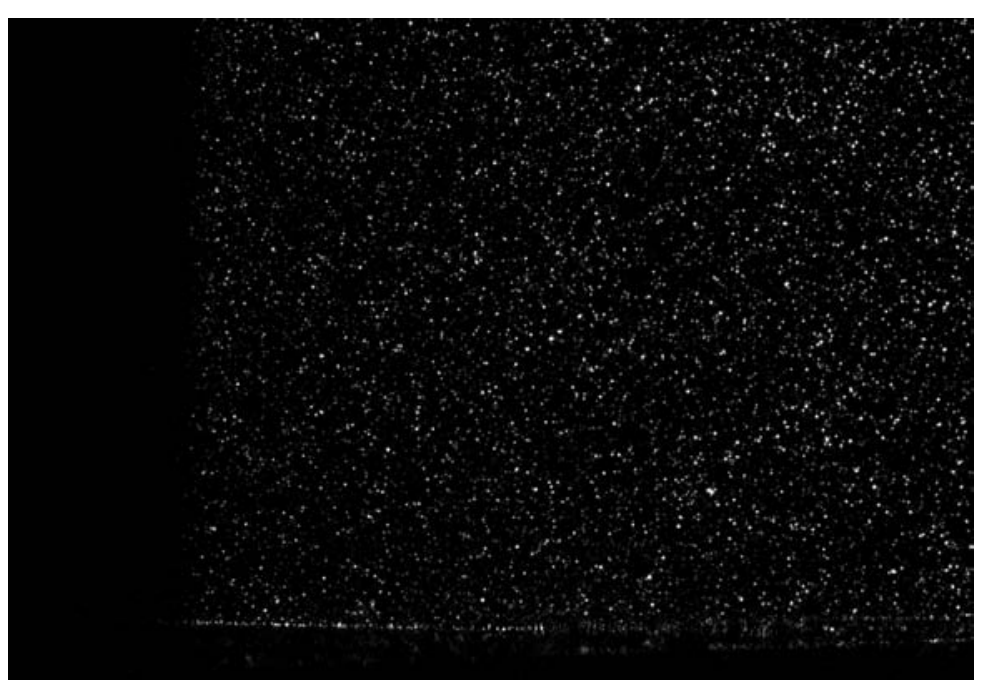

(b)

Figure 8.1: Sample of (a) raw and (b) pre-processed images from camera 1 in the water-channel experiment.

tio of 0.31 and a volume enlargement along the thickness of $40 \%$ in order to increase the quality (as mentioned in chapter 5 ). The final volumes were composed by $82 \times 735 \times 868$ cubic voxels with dimensions of $30 \mu \mathrm{m}$.

For the present water-channel experiment, the particle diameters inside the reconstructed volume were estimated, by means of an autocorrelation procedure according to eq. (3.3.6), as $d_{x}=3.6, d_{y}=3.8$ and $d_{z}=4.0$ voxels. Since no elongation of the reconstructed particles was observed for the present camera arrangement, no volume Gaussian filtering was adopted. This finding can be observed in figure 8.2 displaying the reconstructed volume. This figure shows three orthogonal slices of the normalized reconstructed intensity.

Figure 8.3 presents a normalized intensity profile of a pair of reconstructed volumes for the tomo-PIV processing described. The profiles were normal- 

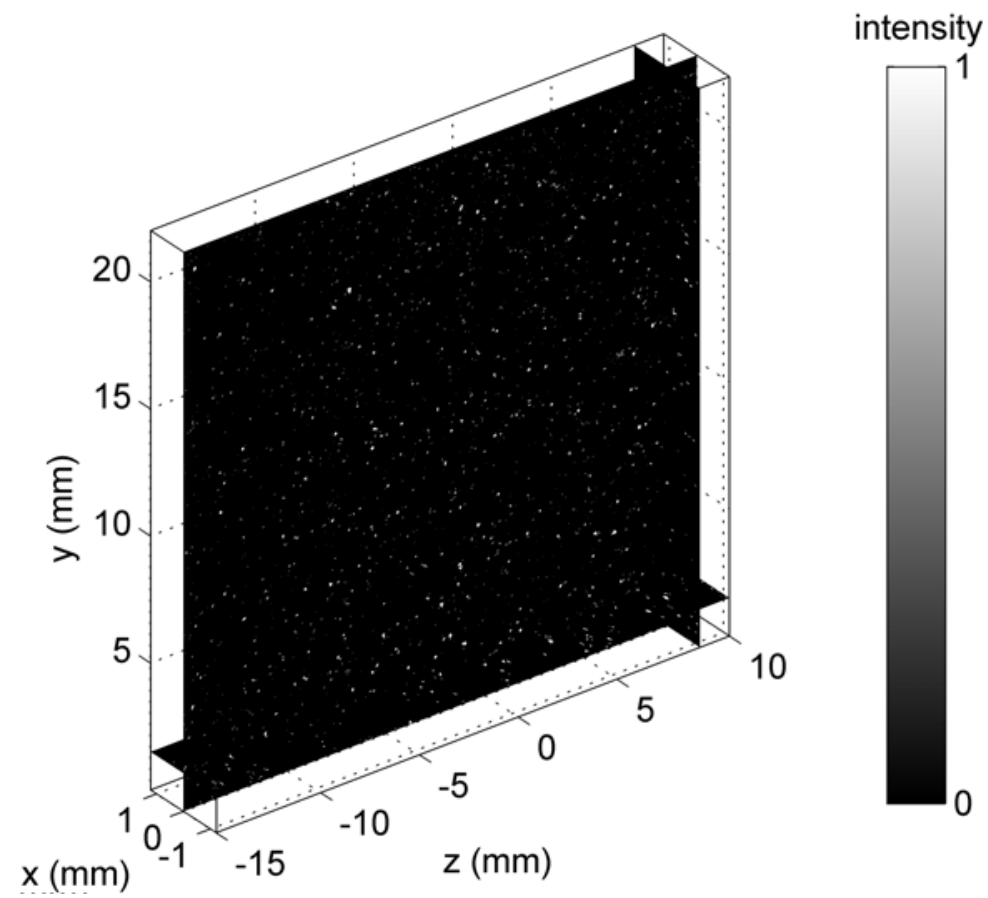

Figure 8.2: Sample of particle intensity inside the tomo-PIV reconstructed volume.

ized by the maximum intensity inside the illuminated region, and are presented as a function of the thickness of the illuminated volume shown in the abscissa (i.e. streamwise direction). A perfect reconstruction of a uniformly illuminated volume should display a distribution of the normalized intensity similar to a top hat. The oscillations of the intensity distribution of figure 8.3 were caused by the small amount of particles inside these instantaneous plots. Contrariwise, an average intensity profile would display a top-hat-like shape. Few instantaneous intensity distributions were used to optimize tomo-PIV parameters before the time-consuming processing of the entire experimental run. Intensity levels outside the illuminated region are associated with ghost particles produced by the reconstruction algorithm. The signal-to-noise ratio, $S N R_{E}$, computed with the average intensity inside the investigation region and the maximum between the two minimums of the profile valleys (one on the right and other on the left side) was 2.5. The normalized variance of the reconstructed intensity, $\sigma_{E}^{*}$, was 21 . Both volume quality indicators satisfied the suggested values for quality reconstructed volumes, possibly leading to a favourable velocity field.

The 3D-3C velocity fields were computed by means of a 3D multi-pass cross-correlation with sub-pixel shift from a pair of volumes containing the particle distribution. After 4 cross-correlation passes, the final interrogation volume had the size of 40x40x40 pixels with $75 \%$ of overlap. The amount of reconstructed particles inside the final interrogation volumes was between 5 to 10 checked by visual inspection. The time delay between pulses was $400 \mu \mathrm{s}$ 


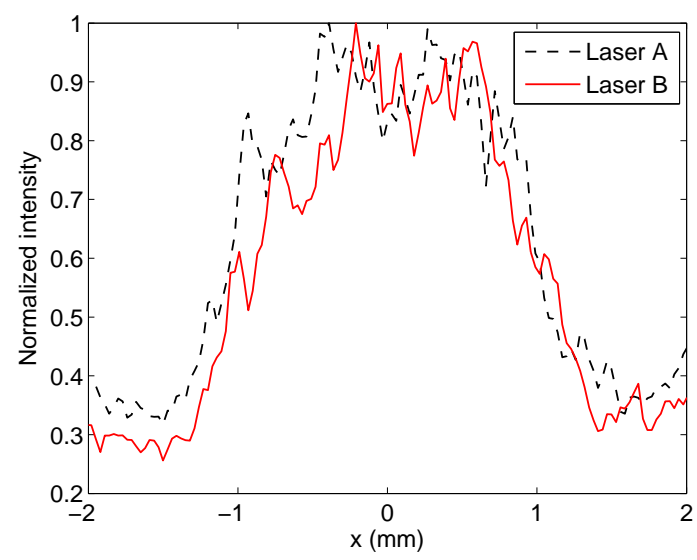

Figure 8.3: Sample of an instantaneous normalized intensity profile of the reconstructed volume for laser pulses $\mathrm{A}$ and $\mathrm{B}$.

allowing for a maximum particle displacement of about 9 voxels between frames (which is equivalent to 6.6 wall units). The final vector field grid was $5 \times 70 \times 83$. The validation of the vectors was performed by means of median filtering, as proposed by [Westerweel, 1994]. The ratio of spurious vectors and the total amount of computed vectors were about $1.8 \%$. The use of larger interrogation volumes created connected regions of spurious vectors that would not be trustful after the vector substitution by the validation procedure. Figure 8.4 presents three orthogonal slices of the velocity fields coloured by magnitude of the streamwise velocity component. In the middle plane, besides the streamwise velocity colormap, $2 \mathrm{D}$ velocity vectors, expressing the wallnormal and spanwise components, are presented. Just one over three vectors are plotted for clarity.

The tomo-PIV processing is time consuming as already stressed in chapter 5. For the water-channel experiment, the computational time to perform the self-calibration process, mentioned in the previous chapter, in the LML cluster with 16 AMD Opteron $61282 \mathrm{GHz}$ processor and $20 \mathrm{~GB}$ of RAM was about $90 \mathrm{~min}$. To reconstruct one volume employing the BIMART algorithm, the required time was $3 \mathrm{~min}$, and to $3 \mathrm{D}$ cross-correlate a pair of reconstructed volumes with 4 passes, $12 \mathrm{~min}$. The time spent to transfer files from the 4 cameras to the acquisition computer (about $3 \mathrm{~h}$ for $4 \times 12$ GB using a hub switch system), from this computer to the LML server and from the server to a post-processing computer should be added to the processing time mentioned. 

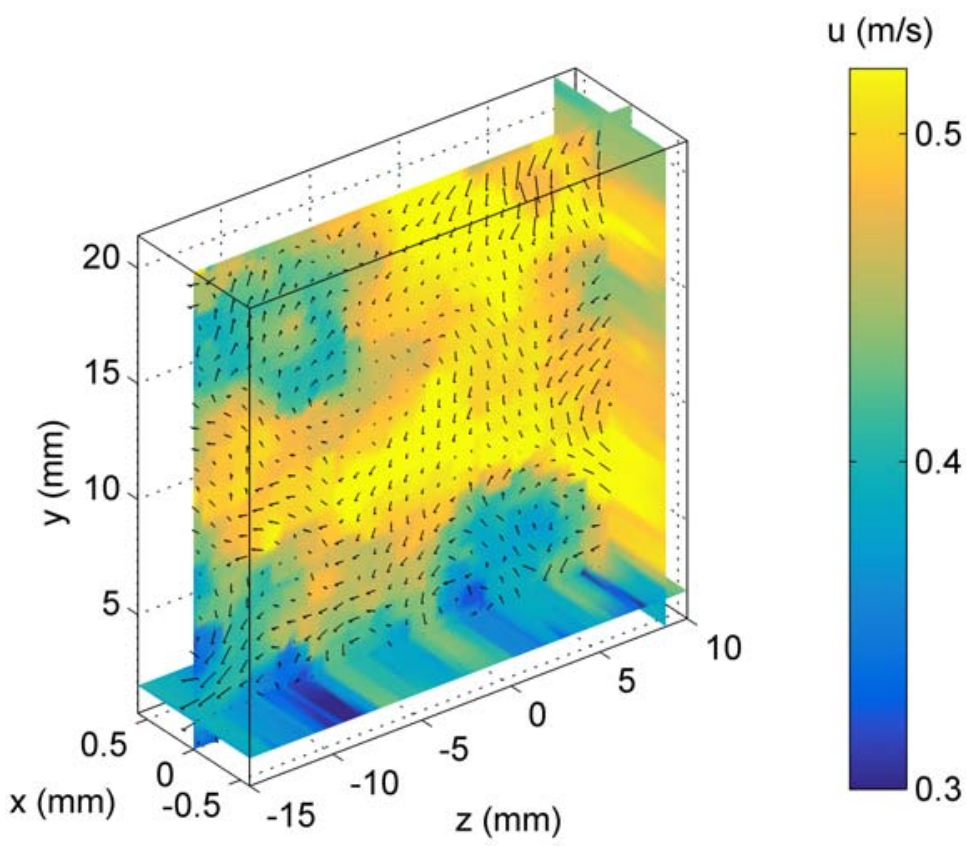

Figure 8.4: Sample of 3D tomo-PIV velocity field in the water-channel experiment. The color code refers to the streamwise velocity. One over three 2D vectors of wall-normal and spanwise velocity components are also plotted.

Table 8.1: Tomo-PIV characteristics of the water-channel experiment.

\begin{tabular}{cccr}
\hline Field of view & Grid space & Vectors per map & Maps \\
\hline $2.5 \times 22 \times 26 \mathrm{~mm}^{3}$ & $0.3 \times 0.3 \times 0.3 \mathrm{~mm}^{3}$ & $5 \times 70 \times 83$ & 510 \\
$61 \times 539 \times 637$ wall units & $7.4 \times 7.4 \times 7.4$ wall units & & \\
\hline
\end{tabular}

Table 8.2: Water-channel flow characteristics.

\begin{tabular}{ccccccc}
\hline$Q(\mathrm{~L} / \mathrm{min})$ & $\bar{U}(\mathrm{~m} / \mathrm{s})$ & $\nu\left(\mathrm{m}^{2} / \mathrm{s}\right)$ & $D_{H}(\mathrm{~mm})$ & $u_{\tau}(\mathrm{m} / \mathrm{s})$ & $R e_{D}$ & $R e_{\tau}$ \\
40.0 & 0.42 & $1.004 \times 10^{-6}$ & 40.0 & 0.0246 & $1.666 \times 10^{4}$ & 490 \\
\hline
\end{tabular}

\subsection{Flow Analysis}

A total of 4080 particle images were reconstructed and their resulting volumes correlated in pairs, producing 510 consecutive vector fields spaced by $0.0025 \mathrm{~s}$ (i.e., an acquisition rate of $400 \mathrm{~Hz}$ ). Table 8.1 summarizes the tomo-PIV experimental characteristics. In this table, the grid space represents the physical distance between two adjacent vectors along $x, y$ and $z$ directions. Table 8.2 compiles the water-channel flow characteristics, such as flow rate $Q$, bulk velocity $\bar{U}$ by eq. (2.3.2), kinematic viscosity $\nu$, hydraulic diameter $D_{H}$, friction velocity $u_{\tau}, R e_{D}$ by eq. (2.3.1) and $R e_{\tau}$ by eq. (2.3.3).

Samples of instantaneous velocity fields at the middle plane of the volume (same slice of the volume displayed in figure 8.4) are presented in figure 8.5. From this figure, the time-resolved tomo-PIV data obtained allowed the 
observation of the typical random-in-appearance behaviour of turbulent flows contrasting with some correlated flow motion along time-adjacent velocity fields. The bottom of the flow seemed to be influenced by the boundary wall located at $y=0$. At this region, the streamwise velocity color and the vector directions changed faster than at the core of the channel. In addition, dark blue regions attached to the bottom wall of velocity fields suggest the existence of crests of low-speed streaks in the present data.

The mean velocity of the flow (figure 8.10) can be subtracted from the instantaneous fields (figure 8.5) to enhance the visualization and comprehension of the channel turbulence. Figure 8.6 shows fluctuating velocity fields at the same plane and at the same time instants as those from the previous figure. Inside the chaotic turbulent flow of this figure, different sizes of vortices could be inferred from the rotatory movement of wall-normal and spanwise fluctuating vectors. The vortices became more clear after the velocity subtraction in agreement with [Adrian et al., 2000a]. For instance, at the instant $0.0100 \mathrm{~s}$, at a position around $(z, y)=(-2.5,2)$, a high velocity fluid (red color code) can be observed moving toward the wall in an apparently sweep event (chapter 2). This event seemed to be a result of counter rotating vortices at $(-5,1)$ and $(-2,1)$, which agrees with observations from the literature ([Robinson, 1991a, Adrian et al., 2000b, Schoppa and Hussain, 2002]).

The measured time-resolved 3D-3C velocity fields allow the calculation of the full velocity gradient tensor. Figure 8.7 presents fluctuating vorticity fields associated with the velocity fields of figure 8.5. It is possible to observe that the streamwise fluctuating vorticity is stronger close to the wall in regions where stronger vortices appeared and were more numerous. Nevertheless, the vorticity contained strong shear regions, commonly found in bounded flows, which makes this flow quantity not recommended for vortex detection. This fact was reported in the literature ([Hunt et al., 1988, Robinson, 1991b, Chakraborty et al., 2005]).

Employing the measured 3D-3C velocity fields, the Q-criterion, eq. (6.2.23), can be computed using the 9 available spatial derivatives. Figure 8.8 shows the same fluctuating velocity fields presented in figure 8.6 coloured by the $Q$ value. This criterion was able to better locate the vortex cores than the vorticity magnitude. Although some misdetection regions of vortices seemed to exist, probably due to the increasing of noise by the spatial derivatives employed in the calculus, the main part of these regions were actual 3D vortices that were not easily visualized within this $y-z$ plane.

Since the water-channel tomo-PIV data from the present experiment were time resolved, 3D plots, with the third dimension being time, complement the 

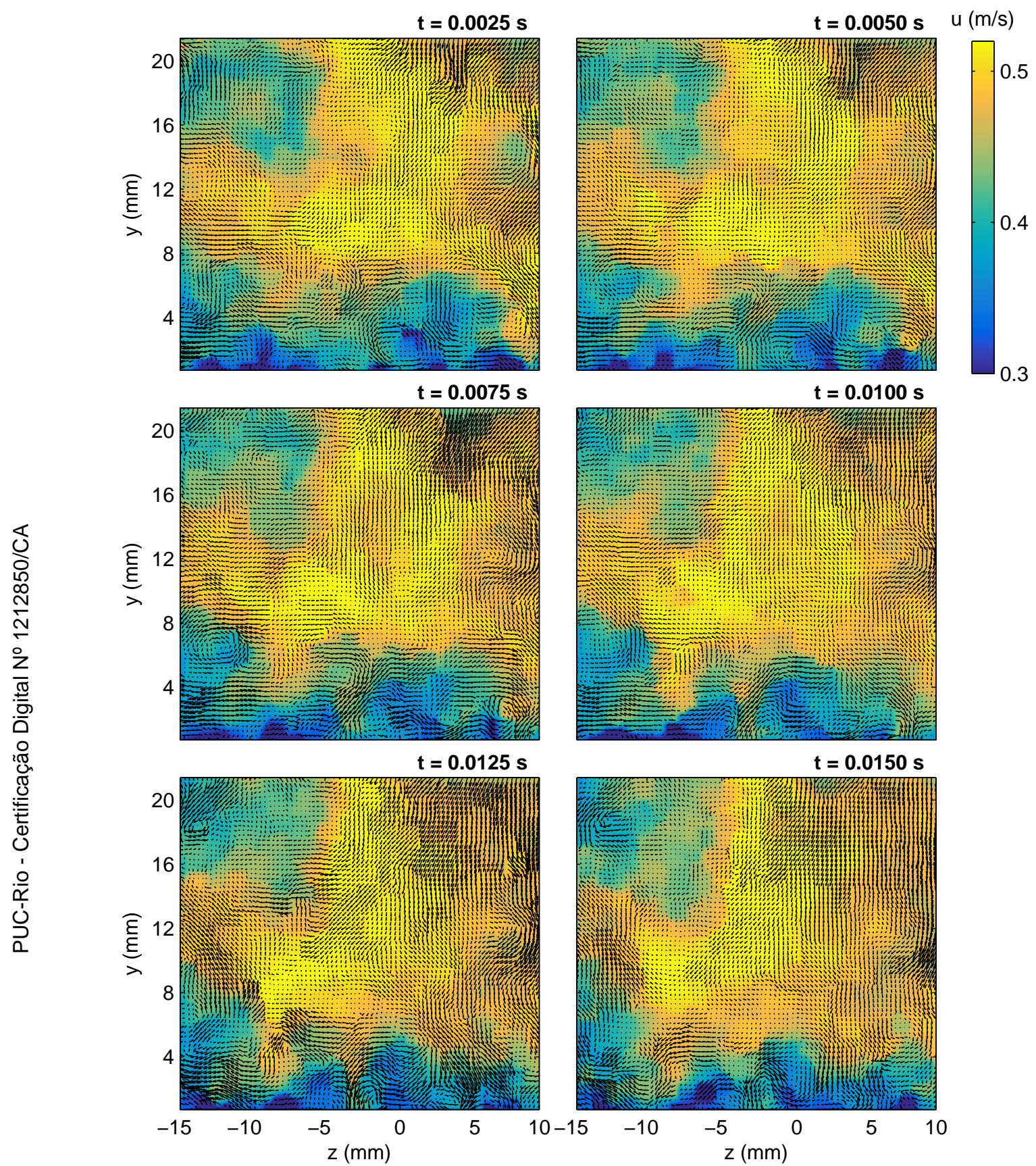

Figure 8.5: Six consecutive instantaneous velocity fields at the middle plane of tomo-PIV measurements. Colour code related to the instantaneous stream-wise velocity and vectors related to the wall-normal and spanwise instantaneous velocity components. 

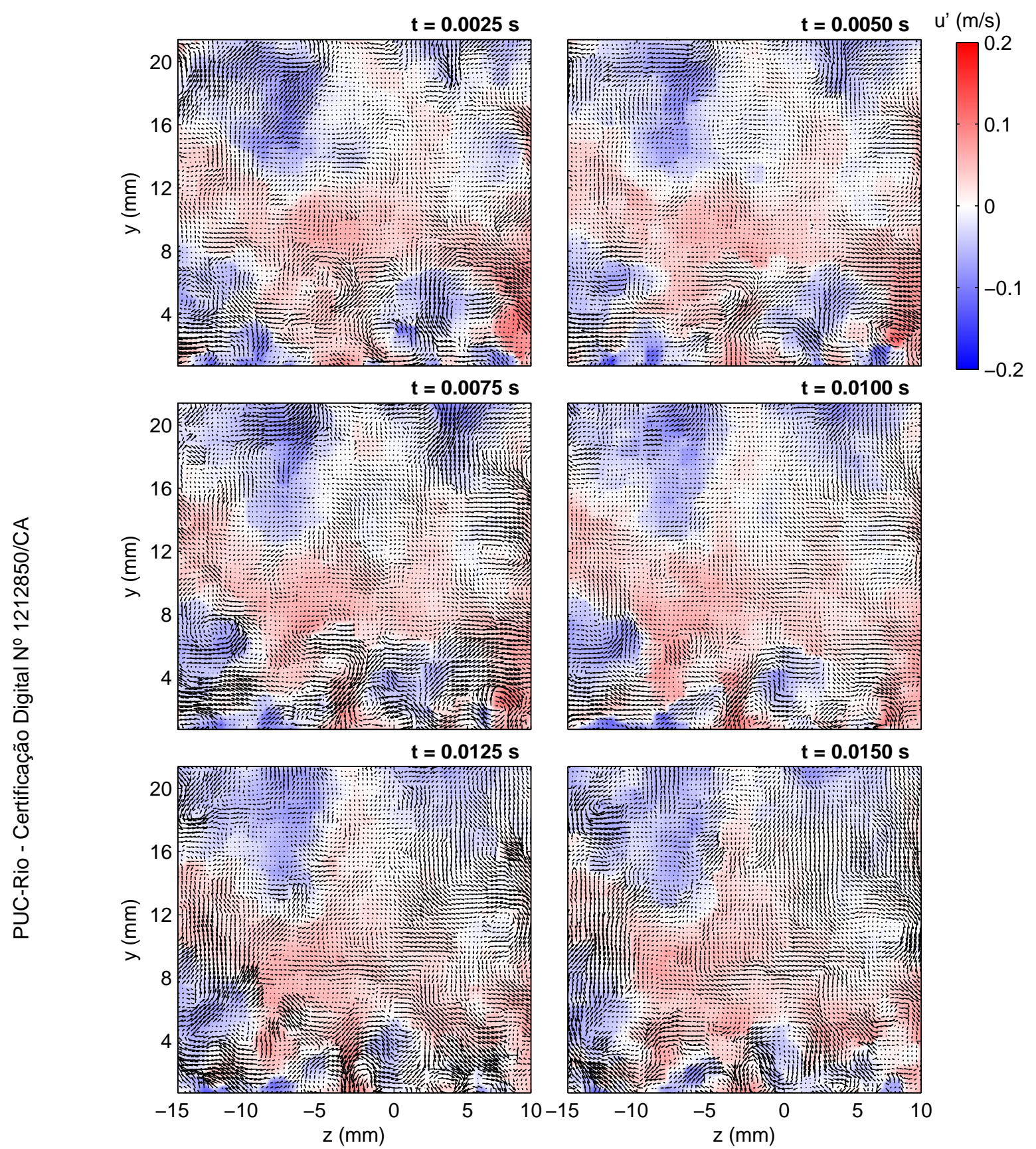

Figure 8.6: Six consecutive fluctuating velocity fields at the middle plane of tomo-PIV measurements. Colour code related to the fluctuating streamwise velocity and vectors related to the wall-normal and spanwise fluctuating velocity components. 


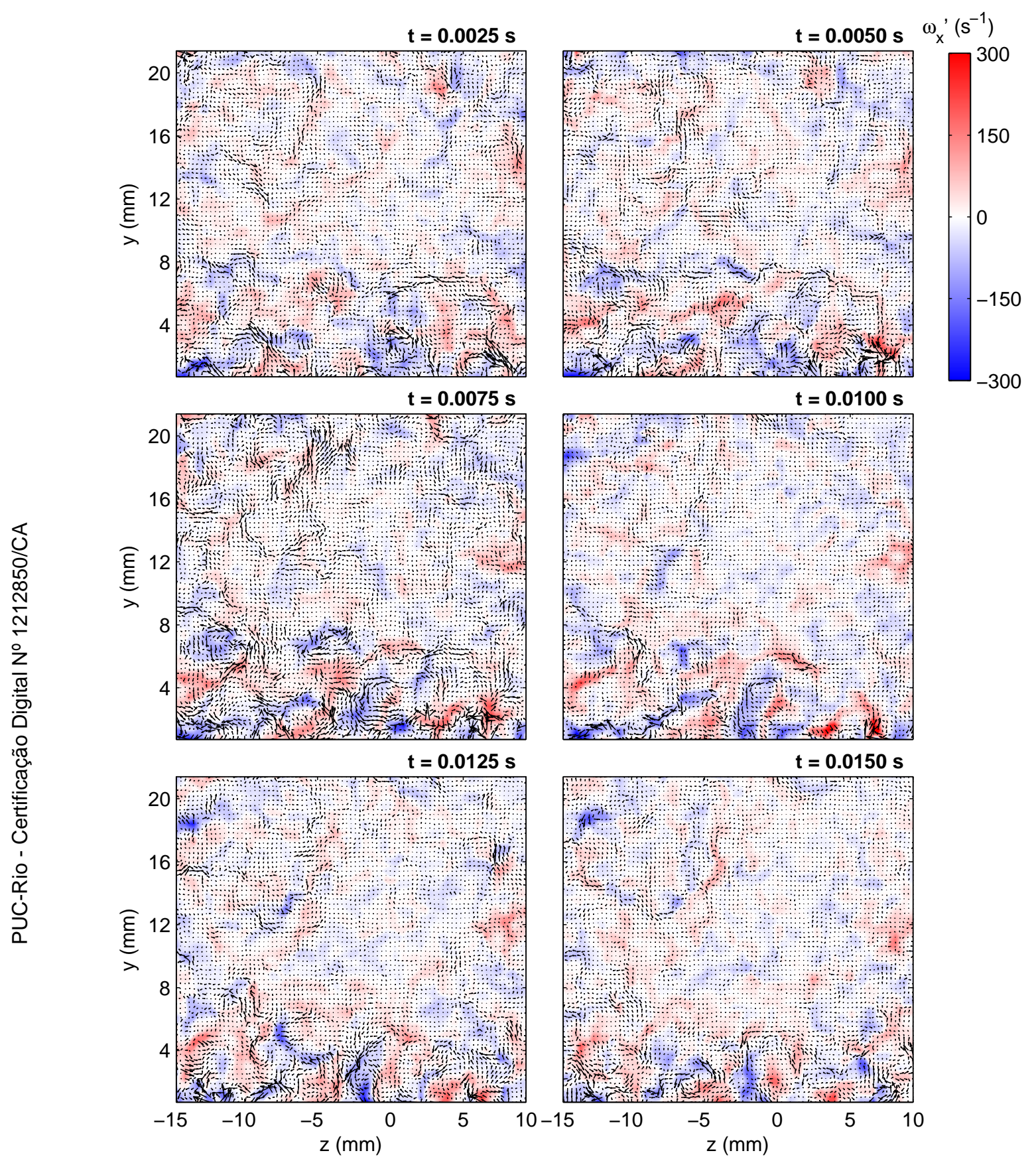

Figure 8.7: Six consecutive fluctuating vorticity fields at the middle plane of tomo-PIV measurements. Colour code related to the fluctuating streamwise vorticity and vectors related to the wall-normal and spanwise fluctuating vorticity components. 

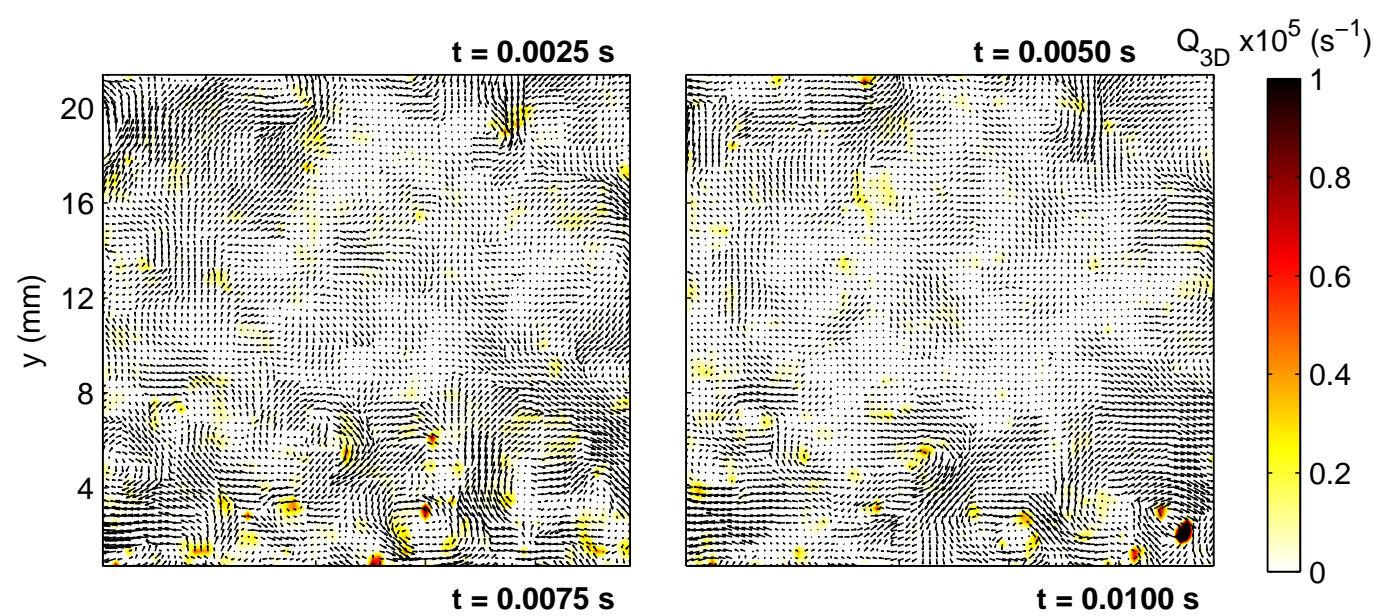

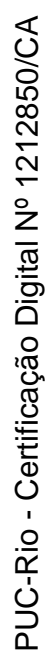
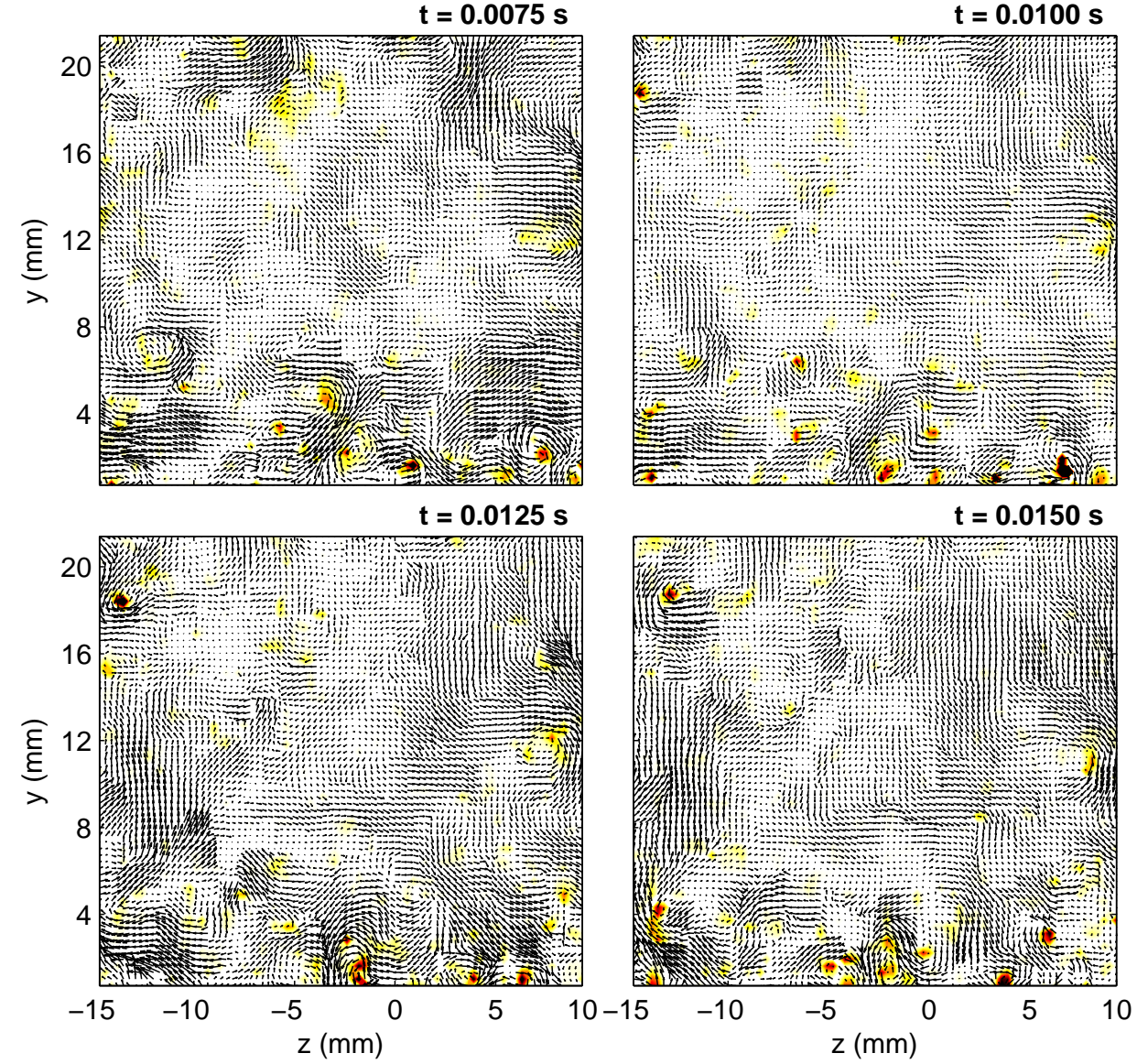

Figure 8.8: Six consecutive fluctuating velocity fields at the middle plane of tomo-PIV measurements. Colour code related to the 3D Q-criterion and vectors related to the wall-normal and spanwise fluctuating velocity components. 


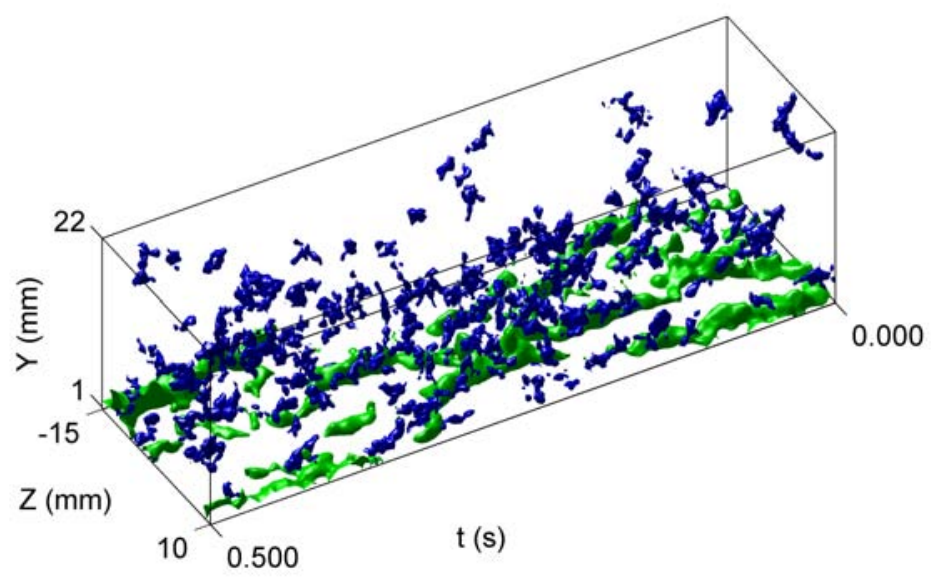

Figure 8.9: Interaction among low-speed streaks (green isosurfaces of $u=0.32 \mathrm{~m} / \mathrm{s}$ ) and turbulent vortical structures (blue isosurfaces of Q-criterion $=500 \mathrm{~s}^{-1}$ ) inside a water channel from tomo-PIV filtered data.

2D analysis, helping in the inference of turbulent structure dynamics and evolution. In favour of the vortical-structure clarification, the elimination of noise in the vortex detection was made by a robust discrete-cosine-transform filter [Garcia, 2010] followed by a volume filtering in the labelled field. The visualizations of low-speed streaks together with vortical structures are displayed in figure 8.9. From this sample, it is possible to identify vortices neighbouring the low-speed streaks. The results, however, still do not present sufficient resolution to allow the distinction of channel-flow turbulence structures.

Although it is recognized that the recorded amount of 510 tomo-PIV vector fields may not seem to be sufficient to produce converged statistics, since this data is correlated in time, preliminary statistical analysis is presented next. Due to time limitations, it was not possible to conduct more data to present results for better converged statistics in the present text.

Figure 8.10(a) displays the mean streamwise velocity field at the middle of the measuring volume. The spanwise axis of the channel coordinate system was corrected taking into account the maximum value of the mean streamwise velocity that should be located at the centre of the channel flow due to the expected symmetry ([Vinuesa et al., 2014]). Channel walls were located at $y=0, y=40, z=-20$ and $z=20 \mathrm{~mm}$. The influence of the walls can be visualized in this colour field, which displays a non-uniform flow along the spanwise direction. This behaviour is in agreement with the direct numerical simulations of [Vinuesa et al., 2014] for ducts with different aspect ratios. They observed three-dimensional effects in the mean flow due to the boundary layers growing along the side-walls of the duct and to secondary motions produced at the duct corners. Figure 8.10 (b) presents the three-dimensional mean flow in a square duct from the DNS of [Vinuesa et al., 2014]. Red rectangle in this 

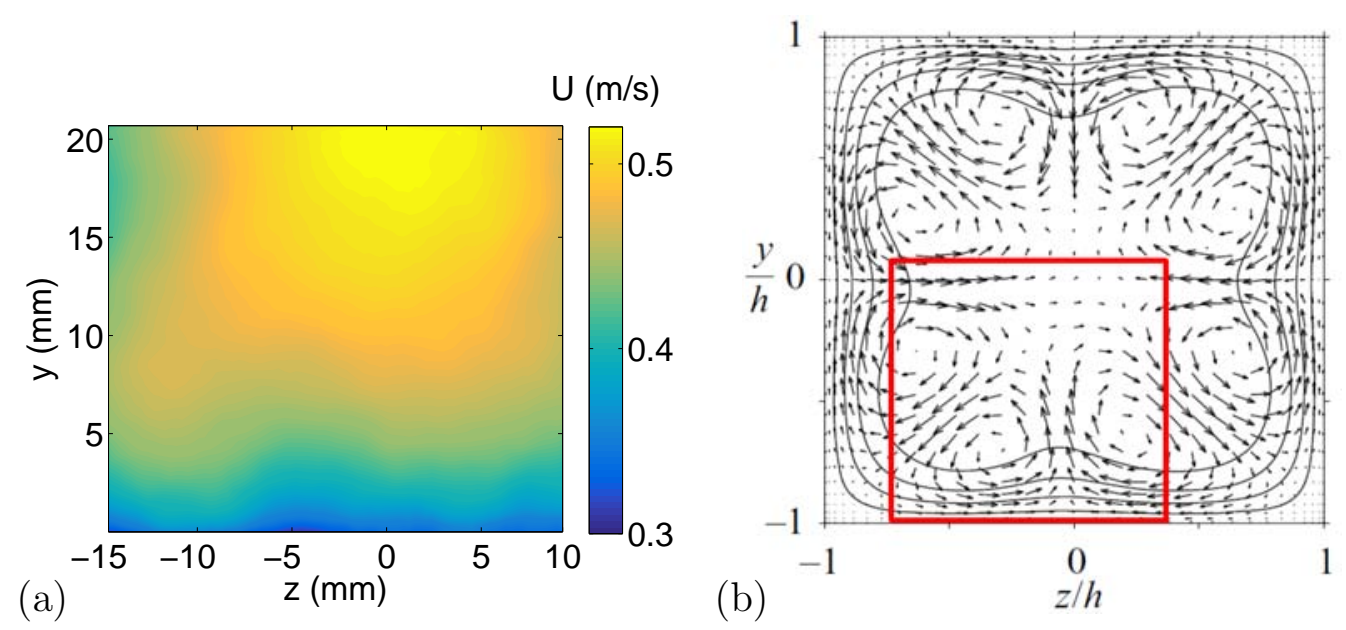

Figure 8.10: (a) Mean streamwise velocity at the middle of the volume. Channel walls were located at $y=0, y=40, z=-20$ and $z=20 \mathrm{~mm}$. (b) Contour lines of the primary mean streamwise flow, and vectors of the secondary mean wall-normal and spanwise flows for the DNS of [Vinuesa et al., 2014]. Red rectangle refers to a similar region of the present tomo-PIV study.

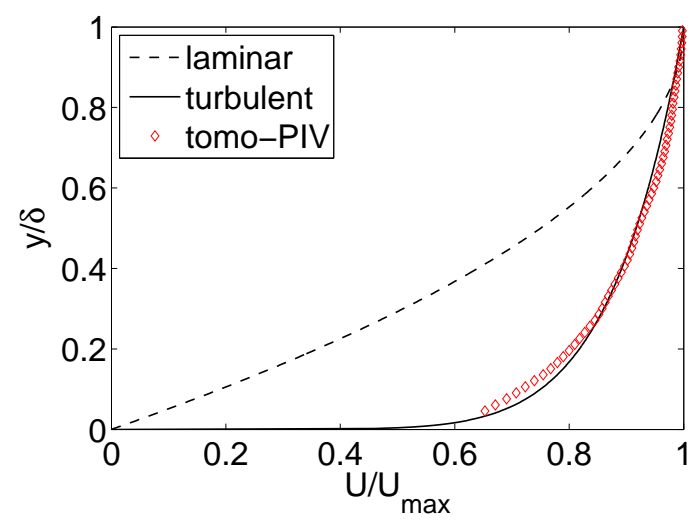

Figure 8.11: Comparison among mean streamwise velocity profiles at the channel centre for tomo-PIV measurements and classical laminar and powerlaw curves.

figure refers to a similar region of the present tomo-PIV study. The secondary motions consist of two counter-rotating vortices in each corner with the flow directed toward the corners along the bisectors. Although this secondary flow is relatively weak compared to the bulk velocity, its effect on the mean velocity distribution is important.

Figure 8.11 presents a comparison among profiles of mean streamwise velocity along wall-normal direction at the centre of the channel $(z=0)$. The quadratic laminar profile was just plotted to show the flatness contrast between turbulence and laminar flows. The tomo-PIV profile agreed with the pipe power-law, eq. (2.3.13) employing $n=8$, although some small differences were observed. Slight shape discrepancy and oscillations were attributed to the small amount of uncorrelated data available to compute the average flow information. 

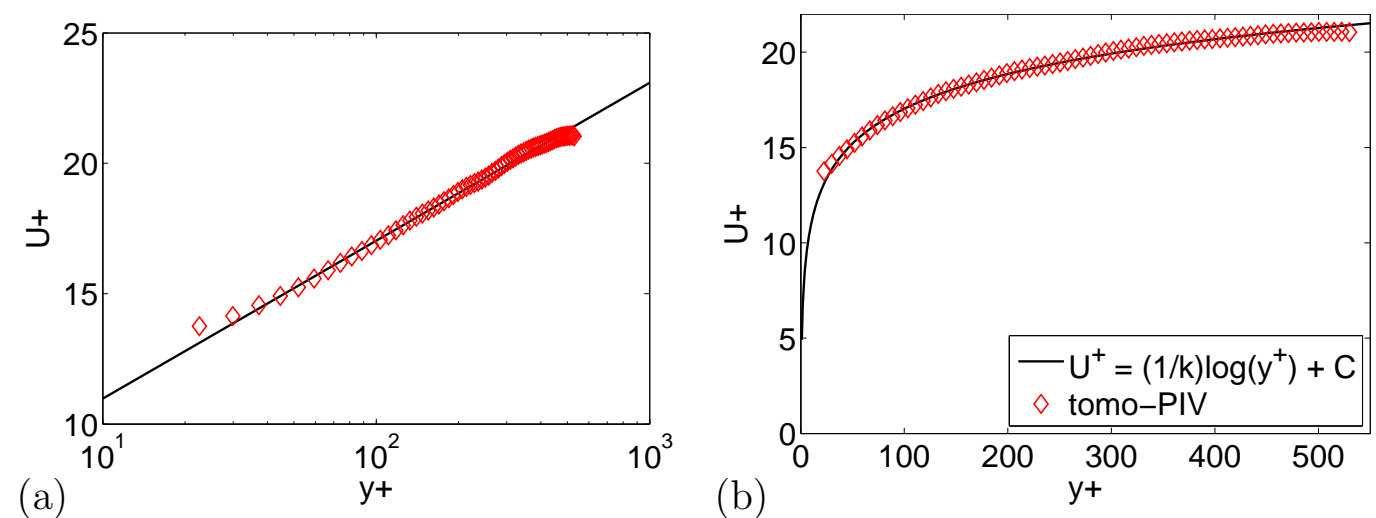

Figure 8.12: Profiles of mean streamwise velocity in (a) semi-log and (b) linear plots in inner scales.

Figure 8.12 presents the streamwise velocity profile at the channel centre in inner scales. Good agreement was observed between the tomo-PIV experimental data and the logarithmic law in both semi-log and linear plots. Slight overestimation of velocity occurred in the two first measurement points probably due to light reflection on the bottom wall.

The water-channel friction velocity $u_{\tau}$ was estimated based on the Clauser method (e.g., [Wei et al., 2005, Kendall, 2006, Kendall and Koochesfahani, 2008]). The equation from table 2.1 was modified to take into account a small possible wall-normal error, $\varepsilon_{y}$, between the physical coordinate system obtained by the calibration procedure and the channel coordinate system.

$$
\frac{U}{u_{\tau}}=\frac{1}{k} \ln \left(\frac{y+\varepsilon_{y}}{\nu / u_{\tau}}\right)+C
$$

The previous equation was rearranged as follows.

$$
U=A^{*} Y^{*}+B^{*}
$$

$$
A^{*}=\frac{u_{\tau}}{k} \quad, \quad Y^{*}=\ln \left(y+\varepsilon_{y}\right) \quad, \quad B^{*}=\frac{u_{\tau}}{k} \ln \left(\frac{u_{\tau}}{\nu}\right)+u_{\tau} C .
$$

A linear regression of the water-channel tomo-PIV data was done employing the eq. (8.2.2). A range of values of the von Kármán empirical constants $(\kappa$ and $B$ ) were considered as suggested by [George, 2007]. The lowest errors between the data and the log-law curve were archived for values of $u_{\tau}=0.0246 \mathrm{~m} / \mathrm{s}, \varepsilon_{y}=0.2 \mathrm{~mm}, k=0.38$ and $C=4.9$.

A second estimation of the friction velocity based on the Blasius' skinfriction coefficient, eq. (2.3.12), was conducted. For a fully-developed channel flow, the skin-friction coefficient, eq. (2.3.9), can be combined with eq. (2.3.12) and eq. (2.2.9) to compute the friction velocity. For the experimental values, the $u_{\tau}$ calculated by Blasius was $0.0245 \mathrm{~m} / \mathrm{s}$. The friction velocities estimated by these two methods remarkably agreed. 


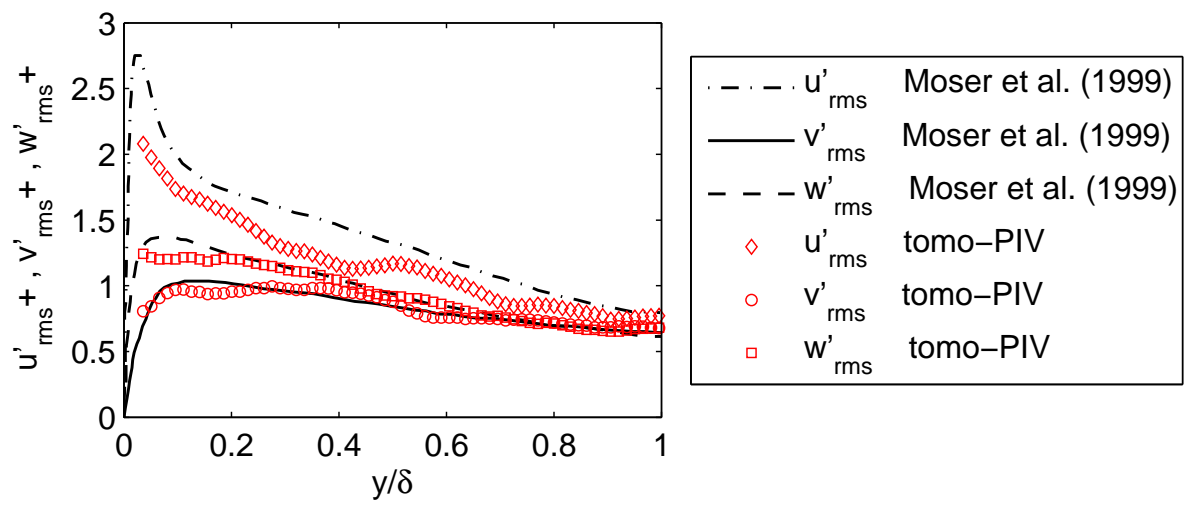

Figure 8.13: Profiles of turbulent velocity fluctuations dimensionless by halfheight of the channel. Comparison with DNS of a channel flow from [Moser et al., 1999].

Figure 8.13 compares velocity statistics from the present tomographicPIV data with the direct numeric simulation (DNS) from [Moser et al., 1999] at $R e_{\tau}$, based on the friction velocity and half-height of the channel, of 590 . The root-mean-square of the fluctuating velocity components scaled by $u_{\tau}$ were plotted with respect to the wall-normal direction normalized by the half-height of the channel. Although the main behaviour of turbulent-fluctuation profiles was captured by the tomo-PIV data, the profiles oscillated and did not coincide with the DNS data. The discrepancies between the two methods seems to be caused predominantly by the insufficient recorded-time to obtain converged statistics, as mentioned previously.

The uncertainty on velocity measurements was estimated based on the root-mean-square of the divergence of the velocity field according to eq. (3.3.9). The mean uncertainty was of the order of 0.11 voxels, which is equivalent to $0.009 \mathrm{~m} / \mathrm{s}$ (i.e. about $1.2 \%$ of the maximum streamwise velocity). This low level of uncertainty was probably achieved due to the measurements performed in a narrow volume and the optimization procedures adopted. This uncertainty value is typical of well-controlled experiments found in the literature ([Scarano and Poelma, 2009, Atkinson et al., 2011]). Figure 8.14 presents the root-meansquare of the divergence and the uncertainty as a function of the wall distance. Closer to the wall, the divergence values were larger, and so were the errors, as a result of difficulties encountered to compute low velocity values at higher shear regions and the contamination of the acquired images by light reflections.

Figure 8.15 presents the probability densities function (PDF) of the three velocity components calculated at about 50 wall units by tomo-PIV. The PDFs display Gaussian distributions, such as those encountered for the boundarylayer experiment (figure 6.14). The marginal asymmetry observed on the PDFs associated to the streamwise and wall-normal fluctuating velocities indicated, 


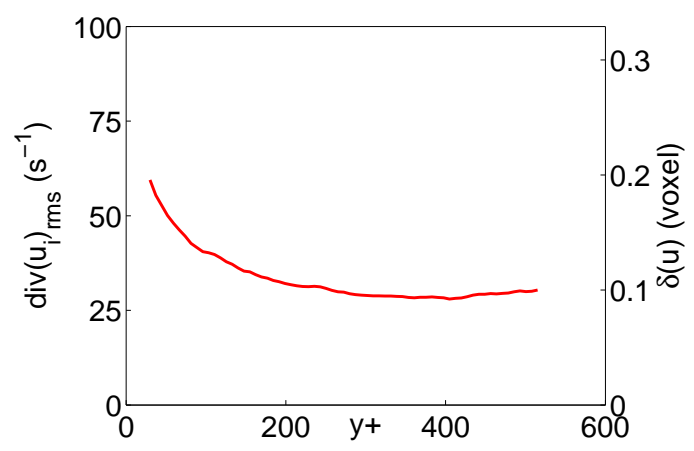

Figure 8.14: Profiles of divergence and uncertainty in velocity.

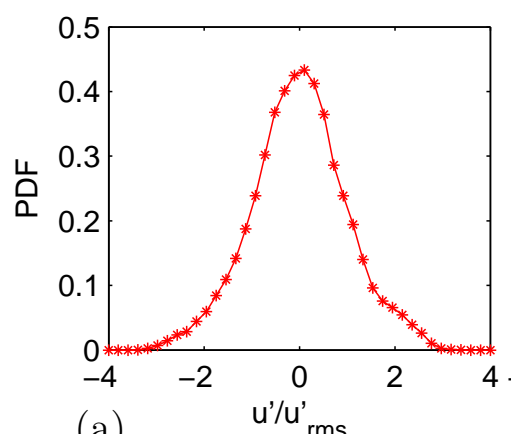

(a)

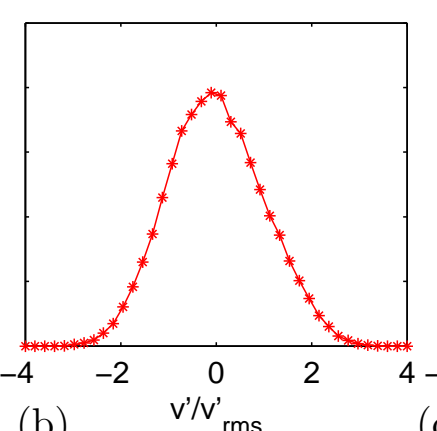

(b)

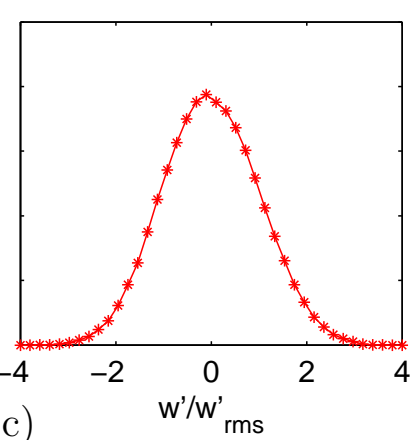

(c)

Figure 8.15: Probability density functions at $y^{+}=50$ for (a) $u^{\prime} / \sqrt{<u^{\prime 2}>}$, (b) $v^{\prime} / \sqrt{\left\langle v^{\prime 2}\right\rangle}$ and (c) $w^{\prime} / \sqrt{\left\langle w^{\prime 2}\right\rangle}$ in the water-channel experiment.

again, lack of statistical convergence.

\subsection{Summary of the Water Channel}

A tomo-PIV technique with four high-speed cameras was applied to study the unsteady character of the near-wall flow in a water-channel with a squared cross-section for a Reynolds number, $R e_{D}$, of 16666 . The results exposed in this chapter clearly indicate the potential of the experiment to allow a comprehensive analysis of the water-channel turbulent structures.

The optimization process applied on the volume illumination, seeding, calibration, image pre-processing, volume reconstruction and cross-correlation was successfully performed. The vector fields generated from BIMART reconstructed volumes resulted in low measurement errors, which were estimated by the velocity-fluctuation divergence.

Samples of instantaneous velocity fields from this time-resolved 3D-3C tomo-PIV presented the random-in-appearance behaviour of turbulent flows with vortices with different scales interacting. The influence of the wall in the formation of more intense and more numbered vortices in this region was also reported. The velocity fields suggested the existence of crests of low-speed streaks in the lower part of the logarithmic region. Other turbulent structures 
were also visible. In especial, the sweep event described seemed to be produced by the neighbouring counter-rotating vortices.

Vortex detection results were demonstrated in terms of vorticity and Q-criterion, which allow the identification of some vortices interacting with low-speed streaks in the near-wall region. The identification of 3D vortices are important, since turbulent flows are unsteady and three-dimensional, which hamper the visualization and interpretation employing standard techniques

Although the amount of data currently processed was insufficient to yield converged statistics, the results obtained presented good agreement between mean streamwise profile and the classical profiles and were able to follow the fluctuating turbulent behaviour of DNS data available from the literature. This is an indication of the quality of the present tomo-PIV experiment. The good quality of the velocity field produced by the tomo-PIV experimental setup of the present work will certainly produce useful statistical data for the interpretation of the turbulent flow characteristics in this square channel flow. To this end, more velocity data needs to be acquired to produce better converged statistics. These additional experiments were not included in the present text due to time constraints. 


\section{9}

\section{Conclusions and Remarks}

The near-wall region of turbulent flows is populated of coherent structures, which are responsible of turbulent energy production, dissipation and transport. Nevertheless, despite the advances in this research field, a number of aspects with respect to the evolution, organization and interaction of these structures remain not completely solved. The objective of the present work was to study near-wall turbulent flows over a smooth flat plate in a wind tunnel and inside a square water channel employing tomo-PIV technique. The characteristics of these turbulent flows and their coherent structures was investigated and quantified in order to provide statistical measurements, which are extremely relevant to validate organization models close to the wall.

Since the performance and accuracy of tomo-PIV technique are sensitive to parametric adjustments, the optimization of the tomo-PIV processing was performed prior to the flow analyses. The optimization of the boundary-layer data processing was investigated based on quality indicators, as light intensity signal-to-noise ratio, normalized intensity variance and reconstruction particle characteristics. The study was motivated by the fact that synthetic data have been constantly employed as a means of guiding the optimization of actual experiments leading, however, to results that do not always present optimal performance. Optimization studies were conducted in the calibration, image preprocessing, volume reconstruction and cross-correlation phases, and proved to be valuable in improving the quality of the velocity measurements.

Image pre-processing algorithms are necessary to correct non-uniform illumination, to improve particle images located near the walls and to reduce sensor noise. For the boundary-layer data, among the different filters tested, subtraction by the time-median image computed from the complete set of instantaneous images, followed by a 3x3 Gaussian filter, yielded the best results. For the water channel flow, an additional filter that removes regions with intensities lower than a threshold value was employed after the background subtraction. This threshold filter performed well decreasing the amount of background noise.

Volume filters are known to improve the quality of reconstructed volumes. 
The volume filters tested were the volume threshold and volume Gaussian filter, both used in the first reconstruction iterations. The tests have shown that a volume filter with a small threshold value can accelerate the convergence and remove ghost particles leading to higher values of the light intensity signal-tonoise ratio and normalized intensity variance quality indicators. The same trend was observed with the Gaussian filter. However, the combination of both filters was seen to decrease the amount of actual particles, reducing the measurement accuracy.

The influence of the number of cameras and particle density on volume reconstruction was discussed. As expected, the quality of reconstruction improved progressively with the number of cameras, with the largest improvement observed when four cameras were used instead of three. Regarding the particle density, the quality of reconstruction decreased with the amount of particles. Nevertheless, the amount of particles is direct linked to velocity field spatial resolution. Therefore, a balance should be sought between the amount of particles and the quality, once the increasing of the number of cameras boosts the system complexity and cost.

A comparative study of four optimized reconstruction algorithms was performed using the boundary-layer data: the standard MART, MART with bilinear weight function, BIMART and SMART. Convergence tests were conducted in order to guarantee that the comparisons were performed for converged results. BIMART and the standard MART yielded the largest light intensity signal-to-noise ratio values, followed by SMART. MART with bilinear function did not yield good results. Except for the latter technique, all algorithms produced about the same particle diameters with low degree of distortion. SMART required about one third, and BIMART half, of the processing time required by MART. Velocity statistics obtained with the different optimized tomo-PIV algorithms were compared with hot-wire data. MART, BIMART and SMART algorithms presented the same level of agreement with the hot-wire data. Likewise, uncertainty levels in the velocity measurements were about the same for the three methods. Well-tuned BIMART and SMART algorithms produced equivalent results to the state-of-the-art MART, with a significant reduction in computational time.

The optimization procedure was successfully applied to the six-camera tomo-PIV measurements in a wall-normal-spanwise volume close to the wall region of a boundary layer. These measurements were taken at a turbulent flow with a momentum-thickness Reynolds number of 8500 inside a wind-tunnel. The camera mapping functions after the self-calibration procedure achieved very low projection and triangulation errors. The vector fields generated from 
BIMART reconstructed volumes resulted in good spatial resolution with low measurement errors.

The present tomo-PIV technique of a turbulent boundary-layer data measured 4-dimensions and 3-components (4D-3C) instantaneous velocity fields, enabling the reconstruction of the time history of the turbulent structures. Instantaneous flow visualization, employing vortex detection based on the Q-criterion, allowed the identification of some vortices interacting with low-speed streaks in the wall boundary layer. The majority of vortices appeared neighbouring the low-speed streaks. The vortices displayed a variety of inclinations and presented a wide range of scales. Low-speed streaks showed branching and meandering behaviours. These findings were in agreement with the literature.

Turbulence statistics from over 8600 vector fields of the boundary-layer data were presented. A good agreement between tomographic PIV and hotwire anemometer data was observed in terms of velocity profiles and turbulent fluctuations. Also, the experimental data matched classical model curves, which reveals that the recording time was sufficient to obtain converge statistics. Likewise, the tomo-PIV data displayed strong agreement with turbulent statistics of a direct numerical simulations from the literature. The probability density functions of the velocity fluctuations from the tomo-PIV method were also compared against the hot-wire anemometer showing superimposed curves.

Differently from stereoscopic PIV, tomo-PIV gives access to the full velocity gradient tensor, which allows the computation of the rate of dissipation and all three vorticity components. The flow dissipation calculated by the tomo-PIV measurements was in agreement with the dissipation computed by the HWA data. A good agreement was also observed among the values of rootmean-square of the fluctuating vorticity from tomographic measurements and direct numerical simulation data. In addition, Kolmogorov length scales can also be obtained from the 4D-3C tomo-PIV data. Results of this length scale matched those obtained from hot-wire measurements.

After the determination of the complete flow statistics, the work focused on the detection and analysis of turbulent structures at the near-wall region of the boundary layer flow. The presence of turbulent structures, namely, low- and high-speed regions, ejections, sweeps and vortices, were detected by means of conditional sampling at the middle plane of the tomo-PIV volume. These structures were classified based on normalized detection functions to account for possible flow quantity changes along the wall-normal direction. The conditional analysis of these coherent structures provides valuable information to develop and validate turbulent models of the near-wall flow behaviour. 
The low- and high-speed conditional statistics revealed that, in average, the low-speed regions moved upwards and the high-speed regions towards the wall. These findings were in agreement with the literature and indicate the strong influence of ejection and sweep events in the mentioned structures. Contrarily, the average of fluctuating velocity components within vortex structures were found to be virtually null, leading to the conclusion that vortices are, in average, convected with the local flow velocity.

Regarding the turbulent fluctuations, low- and high-speed regions, ejections and sweeps display higher values for the root-mean-square of the fluctuating streamwise component than those of the neighbouring flow. The wallnormal velocity fluctuations inside ejections and sweeps were slightly above those obtained from the statistics of the complete flow. For low- and high-speed regions, no appreciable differences were observed between the conditional and complete flow statistics for wall-normal and spanwise turbulent velocity components. The Reynolds stress values calculated within low-speed structures were remarkably higher than those within the high-speed structures, both values being higher than the values computed for the complete flow statistics. Analogous results were found for ejections and sweeps, respectively, which is in agreement with studies from other researchers. These results lead to the conclusion that, among the turbulent structures studied in the present work, ejection and sweep structures, which are associated to low- and high-speed regions, are the major contributors to turbulent production.

No difference was found between the statistics computed at vortex structures and the statistics of the global flow, regarding the turbulent fluctuations and Reynolds stresses. That fact indicates that the vortices are not directly linked to the turbulent production, although they can induce other events that are related to production, as ejection and sweeps.

Low-speed regions and ejections showed slight overestimation of dissipation rate close to the wall compared to the complete flow statistics, while within high-speed regions and sweeps the dissipation profile perfectly agreed with those from the overall flow. However, the elevated uncertainty of this quantity precluded the quantification of these results. The higher dissipation rates were attributed to the higher probability of the existence of vortices inside low-momentum regions than inside high-momentum ones, following the finding of the literature.

As expected, conditional vortex statistics presented dissipation values remarkably above those from the complete flow statistics. Higher dissipation was observed close to the wall, where most intensity vortices were detected. These structures were the main responsible for the turbulent energy dissipation 
in the near-wall region of the boundary-layer flow.

The root-mean-square of the fluctuating vorticity components within lowand high-speed structures, ejections and sweeps presented values that were lower than those from the complete flow statistics, although these differences were within the estimated values for the uncertainty levels. Contrarily, the fluctuating vorticity components of vortex structures were remarkably above those from the entire flow, as expected. The root-mean-square of the streamwise component of vorticity within these structures was found to be significantly higher that the other fluctuating components. This result seemed to be a consequence of the vortex filtering due the orientation of the measurement volume employed for the boundary layer measurements that favours quasi-streamwise vortices.

The displacement orientation of turbulent structures was computed from the fluctuating velocity vector. The probability density functions of projection angles along wall-normal-spanwise plane of low-speed regions started with a bimodal distribution at low wall-normal positions and tended to a single peak distribution with increasing distance from the wall. The peaks, at about $60^{\circ}$ and $-60^{\circ}$, indicated that the main population of low-speed regions were lifted following these directions for the present boundary-layer analysis. These findings suggest to be result of the rotatory movement of turbulent structures neighbouring low-speed regions. High-speed regions showed an analogous behaviour, with initial peaks at about $100^{\circ}$ and $-100^{\circ}$, revealing the main tendency of inward motion of these structures along these directions. For ejection structures, the probability density functions of the streamwise-wallnormal projection angle displayed high occurrence slightly lower than $180^{\circ}$, at about $160^{\circ}$, showing the prevailing direction of upward displacement of ejections. Correspondent inward directions of sweep structures, with peak value of about $-10^{\circ}$, were obtained. The PDFs of wall-normal-spanwise angles presented bimodal distributions with similar peak values for low- and highspeed regions.

Regarding vortical structure geometry, the radii of the streamwise vortices, in average, rise from about 12 to 20 wall units and the standard deviation from 2 to 5 wall units with increasing the distance from the wall, for the studied range. The average vortex centre separation is in the range of 60 to 80 wall units. These results are in agreement with results from the literature employing different detection techniques. The increasing of vortex diameters with wall distance suggest a lift up of the vortices. When scaled by the Kolmogorov length, vortex presented constant mean radius of $5.2 \eta$ and standard deviations of $1.2 \eta$, slightly under the literature values. Statistical results about the geometry of other turbulent structures were not analysed since the amount of 
these type of structures was not sufficient to obtain converged statistics.

The wall-normal-spanwise plane employed in the present investigation favoured the detection of quasi-streamwise vortices among the variety of prevailing vortical structures. The conditional statistics presented are linked to the detection procedure and to the choice of a wall-normal-spanwiseoriented measurement plane. A comparison of the probability density functions of projection and elevation angles with works from the literature were in good agreement considering the filtering effect intrinsic to the experiment. An analysis of the influence of filters, measurement techniques and vortex detection methods was conducted. It was demonstrated that the application of a filter to the data changes the most probable inclination and, because of that, must be use with attention.

The present work showed vortical structures spread all over the flow, nevertheless, they were predominantly found close to low-speed regions and ejections. Therefore they seem to be indirectly correlated with the process of turbulence production in the near-wall region. That is why it is not a surprise that most of turbulent models based on coherent structures on the literature includes vortices. Although, from the present analyses, no single vortex pattern seems representative of the wide variety of shapes and inclinations. Nonetheless, asymmetric vortical structures were the most frequent and the quasi-streamwise vortices displayed similar frequency of occurrence of clockwise and counter-clockwise rotation. The present findings are in line with the turbulent packet proposed by [Schoppa and Hussain, 2000], where an alternating sequence of right and left-handed cane vortices maintains the low-momentum regions. Results are also in agreement with the model proposed by [Adrian et al., 2000b], where low-momentum regions are surrounded by, not only, symmetrical but also asymmetrical hairpins (caneshaped vortices). The preferred asymmetrical-shaped vortices can be explained as a consequence of their faster autogeneration as compared to symmetric hairpins.

The study of the unsteady character of near-wall flows continued in a water-channel with a squared cross-section. This part of the research effort had also the objective of transferring the knowledge of the tomo-PIV technique to the partner laboratory in Brazil and, to best knowledge of the author, it is the first implementation of such technique in the country. The water-channel test section was completely remodelled during the present thesis to allow for the tomo-PIV setup. The experiment employed a four high-speed camera system to measure the velocity field in a wall-normal-spanwise volume close to the wall of a turbulent flow with a Reynolds number based on the hydraulic diameter 
of about 17000. Results obtained in the present work clearly indicate the potential of the experiment to allow a comprehensive analysis of the waterchannel turbulent structures.

The optimization process applied to volume illumination, seeding, calibration, image pre-processing, volume reconstruction and cross-correlation was successfully performed. The vector fields generated from BIMART reconstructed volumes resulted in low measurement uncertainties, similar to those from well executed experiments found in the literature.

Instantaneous visualization from this time-resolved 3D-3C tomo-PIV presented the random-in-appearance behaviour of turbulent flows with vortices with different scales interacting. Other turbulent structures were also observed as a sweep event that seemed to be produced by the neighbouring vortices. The influence of the wall in the formation of stronger in intensity and more numbered vortices in this region was also reported. Visualization of the vortex time-history based on the Q-criterion showed their interaction with low-speed streaks in the near-wall region. The near-wall vortices displayed comparable behaviour for the boundary layer and the channel experiments, in accordance with other researchers.

Although the amount of data currently processed was insufficient to obtain fully converged statistics, the results presented good agreement with the classical mean streamwise profiles and the log-law curve and were able to follow the fluctuating turbulent behaviour of DNS data. This was an indication of the quality of the present tomo-PIV experiments.

In order to analyse the data from both experiments, a 4D-3C data postprocessing tool was developed. The in-house developed software was capable of extracting statistics of the flow and of the turbulent structures from the velocity fields. This software was able to display the data as $2 \mathrm{D}$ and $3 \mathrm{D}$ plots facilitating the understanding of the results and, consequently, the near-wall turbulent flow phenomenon.

The present study produced relevant information regarding the near-wall structure of turbulent flows. The measurement and processing techniques developed are powerful and can potentially be employed to yield new insights into this complex flow phenomena. As a continuation of the work, it is suggested the study of the fluctuating vorticity budget in the logarithmic region. Since the boundary layer flow and its turbulent structures were thoroughly investigated it worth a detailed study of the equation governing the mean fluctuating vorticity field (eq. (6.1) in [Stanislas et al., 2008]).

Another suggestion is the study of the 3D conditional statistics of turbulent structures applied to the LML boundary-layer data. As the tomo-PIV 
velocity fields are time resolved, it is possible to reconstruct the history of turbulent structures as in figure 6.3 and compute the features inside this threedimensional structures, instead of the analysis confined to a plane, as done in the present work. This 3D conditional statistics can deliver more representative quantities of the turbulent structures in addition to their three-dimensional shapes. This analysis was not performed in the present study once the amount of turbulent structures available would not yield fully converged statistics. Nevertheless, there are non processed raw boundary-layer data stored in the LML server that could be used for that purpose.

Regarding the water-channel experiments at PUC-Rio, the present work implemented and validated the tomo-PIV system and processing tools capable of producing high-quality, time-resolved 3D velocity measurements. As a natural sequence to this work, it is suggested that a complete data bank be acquired with information to provide fully converged statistical quantities for a wide range of channel Reynold number values. It should be mentioned that the water channel test section was constructed with the provision to test drag reduction polymers. It is known that a small amount of long-chain flexible polymers dissolved into de fluid can reduce turbulent friction as much as 80\% ([White and Mungal, 2008]). Despite the fact that drag reducing polymer are used in the industry, the effects on the near-wall structures is not fully understood. A comparison of conditional statistics of turbulent structures for flow with and without the addition of polymers can contribute to the understanding of this phenomena. 


\section{0}

\section{Bibliography}

[Achia and Thompson, 1977] Achia, B. and Thompson, D. (1977). Structure of the turbulent boundary in drag-reducing pipe flow. J. Fluid Mech, 81(part 3):439-464.

[Adrian, 1991] Adrian, R. (1991). Particle-imaging techniques for experimental fluid mechanics. Annual review of fluid mechanics, 23(1):261-304.

[Adrian, 1997] Adrian, R. (1997). Dynamic ranges of velocity and spatial resolution of particle image velocimetry. Measurement Science and Technology, 8(12):1393.

[Adrian and Yao, 1985] Adrian, R. and Yao, C.-S. (1985). Pulsed laser technique application to liquid and gaseous flows and the scattering power of seed materials. Applied Optics, 24(1):44-52.

[Álamo et al., 2006] Álamo, J., Jiménez, J., Zandonade, P., and Moser, R. (2006). Self-similar vortex clusters in the turbulent logarithmic region. Journal of Fluid Mechanics, 561:329-358.

[Alec's Web Log, 2014] Alec's Web Log (2014). Posts tagged "colormap". 15October-2014. Accessed 24-July-2016.

http://www.alecjacobson.com/weblog/?tag=colormap.

[Andersen and Kak, 1984] Andersen, A. and Kak, A. (1984). Simultaneous algebraic reconstruction technique (sart): a superior implementation of the art algorithm. Ultrasonic imaging, 6(1):81-94.

[Atkinson et al., 2014] Atkinson, C., Buchmann, N., Amili, O., and Soria, J. (2014). On the appropriate filtering of piv measurements of turbulent shear flows. Experiments in Fluids, 55(1):1-15.

[Atkinson et al., 2011] Atkinson, C., Coudert, S., Foucaut, J.-M., Stanislas, M., and Soria, J. (2011). The accuracy of tomographic particle image velocimetry for measurements of a turbulent boundary layer. Experiments in fluids, 50(4):1031-1056. 
[Atkinson and Soria, 2007] Atkinson, C. and Soria, J. (2007). Algebraic reconstruction techniques for tomographic particle image velocimetry. In 16th Australasian fluid mechanics conference, pages 191-198, Gold Coast, Queensland, Australia.

[Atkinson and Soria, 2009] Atkinson, C. and Soria, J. (2009). An efficient simultaneous reconstruction technique for tomographic particle image velocimetry. Experiments in Fluids, 47(4-5):553-568.

[Aubry et al., 1988] Aubry, N., Holmes, P., Lumley, J., and Stone, E. (1988). The dynamics of coherent structures in the wall region of a turbulent boundary layer. Journal of Fluid Mechanics, 192:115-173.

[Bakewell and Lumley, 1967] Bakewell, H. and Lumley, J. (1967). Viscous sublayer and adjacent wall region in turbulent pipe flow. Physics of Fluids (1958-1988), 10(9):1880-1889.

[Belaid and Mourou, 2011] Belaid, L. and Mourou, W. (2011). Image segmentation: a watershed transformation algorithm. Image Analysis 85 Stereology, 28(2):93-102.

[Bergman et al., 1995] Bergman, L., Rogowitz, B., and Treinish, L. (1995). A rule-based tool for assisting colormap selection. In Proceedings of the $6^{\text {th }}$ conference on Visualization'95, page 118. IEEE Computer Society.

[Bernard et al., 1993] Bernard, P., Thomas, J., and Handler, R. (1993). Vortex dynamics and the production of reynolds stress. Journal of Fluid Mechanics, 253:385-419.

[Bilsky et al., 2011] Bilsky, A., Lozhkin, V., Markovich, D., Tokarev, M., and Shestakov, M. (2011). Optimization and testing of the tomographic method of velocity measurement in the flow volume. Thermophysics and Aeromechanics, 18(4):535-545.

[Bilsky et al., 2012] Bilsky, A., Markovich, D., Tokarev, M., and Lozhkin, V. (2012). Low computation cost reconstruction technique for tomo-piv. In $16^{\text {th }}$ International Symposium on Application of Laser Techniques to Fluid Mechanics, Lisbon, Portugal.

[Birgin et al., 2000] Birgin, E., Martínez, J., and Raydan, M. (2000). Nonmonotone spectral projected gradient methods on convex sets. SIAM Journal on Optimization, 10(4):1196-1211. 
[Blackwelder and Eckelmann, 1979] Blackwelder, R. and Eckelmann, H. (1979). Streamwise vortices associated with the bursting phenomenon. Journal of Fluid Mechanics, 94(03):577-594.

[Blackwelder and Kaplan, 1976] Blackwelder, R. and Kaplan, R. (1976). On the wall structure of the turbulent boundary layer. Journal of Fluid Mechanics, 76(01):89-112.

[Borland and Taylor II, 2007] Borland, D. and Taylor II, R. M. (2007). Rainbow color map (still) considered harmful. IEEE computer graphics and applications, 2:14-17.

[Brown and Thomas, 1977] Brown, G. and Thomas, A. (1977). Large structure in a turbulent boundary layer. Physics of Fluids (1958-1988), 20(10):S243-S252.

[Byrne, 2009] Byrne, C. (2009). Block-iterative algorithms. International Transactions in Operational Research, 16(4):427-463.

[Carlier and Stanislas, 2005] Carlier, J. and Stanislas, M. (2005). Experimental study of eddy structures in a turbulent boundary layer using particle image velocimetry. Journal of Fluid Mechanics, 535(36):143-188.

[Chakraborty et al., 2005] Chakraborty, P., Balachandar, S., and Adrian, R. (2005). On the relationships between local vortex identification schemes. Journal of Fluid Mechanics, 535(2005):189-214.

[Chapman and Tobak, 1985] Chapman, G. and Tobak, M. (1985). Observations, Theoretical Ideas, and Modeling of Turbulent Flows - Past, Present, and Future. Springer.

[Chong et al., 1990] Chong, M., Perry, A. E., and Cantwell, B. (1990). A general classification of three-dimensional flow fields. Physics of Fluids A, $2(5): 765-777$.

[Christensen and Adrian, 2001] Christensen, K. and Adrian, R. (2001). Statistical evidence of hairpin vortex packets in wall turbulence. Journal of Fluid Mechanics, 431:433-443.

[Christensen and Adrian, 2002] Christensen, K. and Adrian, R. (2002). Measurement of instantaneous eulerian acceleration fields by particle image accelerometry: method and accuracy. Experiments in fluids, 33(6):759-769. 
[Corino and Brodkey, 1969] Corino, E. and Brodkey, R. (1969). A visual investigation of the wall region in turbulent flow. Journal of Fluid Mechanics, $37(01): 1-30$.

[de Silva et al., 2013] de Silva, C., Baidya, R., and Marusic, I. (2013). Enhancing tomo-piv reconstruction quality by reducing ghost particles. Measurement Science and Technology, 24(2):024010.

[Dekou et al., 2016] Dekou, R., Foucaut, J.-M., Roux, S., and Stanislas, M. (2016). Large-scale organization of a near-wall turbulent boundary layer. In Progress in Wall Turbulence 2, pages 335-346. Springer.

[del Álamo et al., 2006] del Álamo, J., Jiménez, J., Zandonade, P., and Moser, R. (2006). Self-similar vortex clusters in the turbulent logarithmic region. Journal of Fluid Mechanics, 561:329-358.

[Delo et al., 2004] Delo, C., Kelso, R., and Smits, A. (2004). Threedimensional structure of a low-reynolds-number turbulent boundary layer. Journal of Fluid Mechanics, 512:47-83.

[Dennis and Nickels, 2011a] Dennis, D. and Nickels, T. (2011a). Experimental measurement of large-scale three-dimensional structures in a turbulent boundary layer. part 1. vortex packets. Journal of Fluid Mechanics, 673:180217.

[Dennis and Nickels, 2011b] Dennis, D. and Nickels, T. (2011b). Experimental measurement of large-scale three-dimensional structures in a turbulent boundary layer. part 2. long structures. Journal of Fluid Mechanics, 673:218-244.

[Discetti and Astarita, 2012] Discetti, S. and Astarita, T. (2012). A fast multiresolution approach to tomographic piv. Experiments in fluids, 52(3):765777 .

[Discetti and Astarita, 2013] Discetti, S. and Astarita, T. (2013). Stability of single image self-calibration for tomographic piv. In PIV'13 - $10^{\text {th }}$ International Symposium on Particle Image Velocimetry, Delft, The Netherlands.

[Discetti et al., 2013] Discetti, S., Natale, A., and Astarita, T. (2013). Spatial filtering improved tomographic piv. Experiments in fluids, 54(4):1-13.

[Earl et al., 2015] Earl, T., Jeon, Y., Lecordier, B., and David, L. (2015). Accuracy and speed assessment of $3 \mathrm{~d}$ cross-correlation algorithms for twoframe and multi-frame piv. In $11^{\text {th }}$ International Symposium on Particle Image Velocimetry - PIV15, Santa Barbara, USA. 
[Elsinga, 2008] Elsinga, G. (2008). Tomographic particle image velocimetry and its application to turbulent boundary layers. PhD thesis, TU Delft, Delft University of Technology.

[Elsinga et al., 2010] Elsinga, G., Adrian, R., Van Oudheusden, B., and Scarano, F. (2010). Three-dimensional vortex organization in a highreynolds-number supersonic turbulent boundary layer. Journal of Fluid Mechanics, 644:35-60.

[Elsinga et al., 2005a] Elsinga, G., Scarano, F., Wieneke, B., and van Oudheusden, B. (2005a). Assessment of tomo-piv for three-dimensional flows. In $6^{\text {th }}$ International Symposium on Particle Image Velocimetry, Pasadena, California, USA.

[Elsinga et al., 2005b] Elsinga, G., Scarano, F., Wieneke, B., and van Oudheusden, B. (2005b). Tomographic particle image velocimetry. In $6^{\text {th }}$ International Symposium on Particle Image Velocimetry, Pasadena, California, USA.

[Elsinga et al., 2006] Elsinga, G., Scarano, F., Wieneke, B., and van Oudheusden, B. (2006). Tomographic particle image velocimetry. Experiments in Fluids, 41(6):933-947.

[Elsinga and Tokgoz, 2014] Elsinga, G. and Tokgoz, S. (2014). Ghost hunting - an assessment of ghost particle detection and removal methods for tomographic-piv. Measurement Science and Technology, 25(8):084004.

[Elsinga et al., 2011] Elsinga, G., Westerweel, J., Scarano, F., and Novara, M. (2011). On the velocity of ghost particles and the bias errors in tomographicpiv. Experiments in fluids, 50(4):825-838.

[Foucaut et al., 2004] Foucaut, J.-M., Carlier, J., and Stanislas, M. (2004). Piv optimization for the study of turbulent flow using spectral analysis. Measurement Science and Technology, 15(6):1046.

[Foucaut et al., 2011a] Foucaut, J.-M., Coudert, S., Avelar, A., Lecordier, B., Godard, G., Gobin, C., Thomas, L., Braud, P., and David, L. (2011a). Experiment of high repetition tomographic piv in a high reynolds number turbulent boundary layer wind tunnel. In PIV'11 - Ninth International Symposium on Particle Image Velocimetry.

[Foucaut et al., 2012a] Foucaut, J.-M., Coudert, S., Avelar, A., Lecordier, B., Godard, G., Gobin, C., Thomas, L., Braud, P., David, L., and Tremblais, 
B. (2012a). Une expérience de piv tomographique à haute cadence dans une couche limite turbulente à grand nombre de reynolds. In 13ième Congrès Francophone de Techniques Laser, Rouen, France.

[Foucaut et al., 2012b] Foucaut, J.-M., Coudert, S., Braud, C., and Velte, C. (2012b). Large field spiv using separated light sheets in a spanwise plane applied to a turbulent boundary layer with vortex generators. In $16^{\text {th }}$ International Symposium on Applications of Laser Techniques to Fluid Mechanics.

[Foucaut et al., 2011b] Foucaut, J.-M., Coudert, S., Stanislas, M., and Delville, J. (2011b). Full 3d correlation tensor computed from double field stereoscopic piv in a high reynolds number turbulent boundary layer. Experiments in fluids, 50(4):839-846.

[Foucaut et al., 2016] Foucaut, J.-M., Cuvier, C., Stanislas, M., and George, W. K. (2016). Quantification of the full dissipation tensor from an l-shaped spiv experiment in the near wall region. In Progress in Wall Turbulence 2, pages 429-439. Springer.

[Foucaut and Stanislas, 2002] Foucaut, J.-M. and Stanislas, M. (2002). Some considerations on the accuracy and frequency response of some derivative filters applied to piv vector fields. Measurement Science and Technology, 13(7):1058-1071.

[Fox et al., 1998] Fox, R. W., McDonald, A., and Pritchard, P. J. (1998). Introduction to fluid mechanics, volume 5. John Wiley \& Sons New York.

[Fukuchi, 2012] Fukuchi, Y. (2012). Influence of number of cameras and preprocessing for thick volume tomographic piv. In $16^{\text {th }}$ International Symposium on Applications of Laser Techniques to Fluid Mechanics.

[Gao et al., 2011] Gao, Q., Ortiz-Duenas, C., and Longmire, E. (2011). Analysis of vortex populations in turbulent wall-bounded flows. Journal of Fluid Mechanics, 678:87-123.

[Gao et al., 2013] Gao, Q., Ortiz-Dueñas, C., and Longmire, E. (2013). Evolution of coherent structures in turbulent boundary layers based on moving tomographic piv. Experiments in fluids, 54(12):1-16.

[Garcia, 2010] Garcia, D. (2010). Robust smoothing of gridded data in one and higher dimensions with missing values. Computational Statistics $\&$ Data Analysis, 54(4):1167-1178. 
[George, 2007] George, W. (2007). Is there a universal log law for turbulent wall-bounded flows? Philosophical Transactions of the Royal Society of London A: Mathematical, Physical and Engineering Sciences, 365(1852):789806.

[George, 2013] George, W. (2013). Lectures in turbulence for the $21^{\text {st }}$ century. University Lecture.

[Gesemann et al., 2010] Gesemann, S., Schanz, D., Schröder, A., Petra, S., and Schnörr, C. (2010). Recasting tomo-piv reconstruction as constrained and 11-regularized non-linear least squares problem. In $15^{\text {th }}$ International Symposium on Applications of Laser Techniques to Fluid Mechanics, Lisbon, Portugal.

[Gonzalez, 2009] Gonzalez, R. (2009). Digital image processing. Pearson Education India.

[Gordon et al., 1970] Gordon, R., Bender, R., and Herman, G. (1970). Algebraic reconstruction techniques (art) for three-dimensional electron microscopy and x-ray photography. Journal of theoretical Biology, 29(3):471-481.

[Grass, 1971] Grass, A. (1971). Structural features of turbulent flow over smooth and rough boundaries. Journal of Fluid Mechanics, 50(02):233-255.

[Guala et al., 2006] Guala, M., Hommema, S., and Adrian, R. (2006). Largescale and very-large-scale motions in turbulent pipe flow. Journal of Fluid Mechanics, 554:521-542.

[Guezennec et al., 1989] Guezennec, Y., Piomelli, U., and Kim, J. (1989). On the shape and dynamics of wall structures in turbulent channel flow. Physics of Fluids A: Fluid Dynamics (1989-1993), 1(4):764-766.

[Gupta et al., 1971] Gupta, A., Laufer, J., and Kaplan, R. (1971). Spatial structure in the viscous sublayer. Journal of Fluid Mechanics, 50(03):493512 .

[Hartnett et al., 1962] Hartnett, J., Koh, J., and McComas, S. (1962). A comparison of predicted and measured friction factors for turbulent flow through rectangular ducts. Journal of Heat Transfer, 84(1):82-88.

[Head and Bandyopadhyay, 1981] Head, M. and Bandyopadhyay, P. (1981). New aspects of turbulent boundary-layer structure. Journal of Fluid Mechanics, 107:297-338. 
[Herman and Lent, 1976] Herman, G. and Lent, A. (1976). Iterative reconstruction algorithms. Computers in biology and medicine, 6(4):273-294.

[Herpin et al., 2013] Herpin, S., Stanislas, M., Foucaut, J.-M., and Coudert, S. (2013). Influence of the reynolds number on the vortical structures in the logarithmic region of turbulent boundary layers. Journal of Fluid Mechanics, $716: 5-50$.

[Herpin et al., 2010] Herpin, S., Stanislas, M., and Soria, J. (2010). The organization of near-wall turbulence: a comparison between boundary layer spiv data and channel flow dns data. Journal of Turbulence, 11.

[Herpin et al., 2008] Herpin, S., Wong, C., Stanislas, M., and Soria, J. (2008). Stereoscopic piv measurements of a turbulent boundary layer with a large spatial dynamic range. Experiments in fluids, 45(4):745-763.

[Hinze, 1975] Hinze, J. (1975). Turbulence. McGraw Hill, New York.

[Holmes et al., 1998] Holmes, P., Lumley, J., and Berkooz, G. (1998). Turbulence, coherent structures, dynamical systems and symmetry. Cambridge University Press.

[Honkan and Andreopoulos, 1997] Honkan, A. and Andreopoulos, Y. (1997). Vorticity, strain-rate and dissipation characteristics in the near-wall region of turbulent boundary layers. Journal of Fluid Mechanics, 350:29-96.

[Hori and Sakakibara, 2004] Hori, T. and Sakakibara, J. (2004). High-speed scanning stereoscopic piv for $3 \mathrm{~d}$ vorticity measurement in liquids. Measurement Science and Technology, 15(6):1067.

[Hoyas and Jiménez, 2008] Hoyas, S. and Jiménez, J. (2008). Reynolds number effects on the reynolds-stress budgets in turbulent channels. Physics of Fluids, 20(10):101511.

[Hulst and Van De Hulst, 1957] Hulst, H. and Van De Hulst, H. (1957). Light scattering by small particles. Courier Corporation.

[Hunt et al., 1988] Hunt, J., Wray, A., and Moin, P. (1988). Eddies, streams, and convergence zones in turbulent flows. In Studying Turbulence Using Numerical Simulation Databases, 2, volume 1, pages 193-208.

[Hutchins and Marusic, 2007] Hutchins, N. and Marusic, I. (2007). Evidence of very long meandering features in the logarithmic region of turbulent boundary layers. Journal of Fluid Mechanics, 579:1-28. 
[Jeong and Hussain, 1995] Jeong, J. and Hussain, F. (1995). On the identification of a vortex. Journal of fluid mechanics, 285:69-94.

[Jeong et al., 1997] Jeong, J., Hussain, F., Schoppa, W., and Kim, J. (1997). Coherent structures near the wall in a turbulent channel flow. Journal of Fluid Mechanics, 332:185-214.

[Jiménez and Lozano-Durán, 2016] Jiménez, J. and Lozano-Durán, A. (2016). Coherent structures in wall-bounded turbulence. In Progress in Wall Turbulence 2, pages 37-46. Springer.

[Jiménez et al., 1993] Jiménez, J., Wray, A., Saffman, P., and Rogallo, R. (1993). The structure of intense vorticity in isotropic turbulence. Journal of Fluid Mechanics, 255:65-90.

[Jodai et al., 2014] Jodai, Y., Westerweel, J., and Elsinga, G. (2014). Timeresolved tomographic-piv measurement in the near-wall region of a turbulent boundary layer. In $1^{\text {th }}$ International Symposium on Applications of Laser Techniques to Fluid Mechanics, Lisbon, Portugal.

[Johansson et al., 1991] Johansson, A., Alfredsson, P., and Kim, J. (1991). Evolution and dynamics of shear-layer structures in near-wall turbulence. Journal of Fluid Mechanics, 224:579-599.

[Kähler, 2004] Kähler, C. (2004). Investigation of the spatio-temporal flow structure in the buffer region of a turbulent boundary layer by means of multiplane stereo piv. Experiments in Fluids, 36(1):114-130.

[Kähler and Kompenhans, 2000] Kähler, C. and Kompenhans, J. (2000). Fundamentals of multiple plane stereo particle image velocimetry. Experiments in Fluids, 29(1):S070-S077.

[Kähler et al., 2016] Kähler, C. J., Astarita, T., Vlachos, P. P., Sakakibara, J., Hain, R., Discetti, S., Foy, R., and Cierpka, C. (2016). Main results of the 4th international piv challenge. Experiments in Fluids, 57(6):1-71.

[Kang et al., 2007] Kang, S.-J., Tanahashi, M., and Miyauchi, T. (2007). Dynamics of fine scale eddy clusters in turbulent channel flows. Journal of Turbulence, 8:N52.

[Kendall, 2006] Kendall, A. (2006). A method for estimating wall friction in turbulent boundary layers. In $25^{\text {th }}$ AIAA Aerodynamic Measurement Technology and Ground Testing Conference. San Francisco: American Institute of Aeronautics and Astronautics, volume 3834. 
[Kendall and Koochesfahani, 2008] Kendall, A. and Koochesfahani, M. (2008). A method for estimating wall friction in turbulent wall-bounded flows. Experiments in Fluids, 44(5):773-780.

[Kim et al., 1971] Kim, H., Kline, S., and Reynolds, W. (1971). The production of turbulence near a smooth wall in a turbulent boundary layer. Journal of Fluid Mechanics, 50(01):133-160.

[Kim et al., 1987] Kim, J., Moin, P., and Moser, R. (1987). Turbulence statistics in fully developed channel flow at low reynolds number. Journal of fluid mechanics, 177:133-166.

[Kim and Adrian, 1999] Kim, K. and Adrian, R. (1999). Very large-scale motion in the outer layer. Physics of Fluids (1994-present), 11(2):417-422.

[Klebanoff et al., 1962] Klebanoff, P., Tidstrom, K., and Sargent, L. (1962). The three-dimensional nature of boundary-layer instability. Journal of Fluid Mechanics, 12(01):1-34.

[Kline et al., 1967] Kline, S., Reynolds, W., Schraub, F., and Runstadler, P. (1967). The structure of turbulent boundary layers. Journal of Fluid Mechanics, 30(04):741-773.

[Kline and Runstadler, 1958] Kline, S. and Runstadler, P. (1958). Some Preliminary Results of Visual Studies on the Flow Model of the Wall Layers of the Turbulent Boundary Layer. United States Air Force, Office of Scientific Research.

[Koltracht and Lancaster, 1990] Koltracht, I. and Lancaster, P. (1990). Contraining strategies for linear iterative processes. IMA Journal of Numerical Analysis, 10(4):555-567.

[Lagraa et al., 2004] Lagraa, B., Labraga, L., and Mazouz, A. (2004). Characterization of low-speed streaks in the near-wall region of a turbulent boundary layer. European Journal of Mechanics-B/Fluids, 23(4):587-599.

[Lamarche and Leroy, 1990] Lamarche, F. and Leroy, C. (1990). Evaluation of the volume of intersection of a sphere with a cylinder by elliptic integrals. Computer Physics Communications, 59(2):359-369.

[Landahl, 1990] Landahl, M. (1990). On sublayer streaks. Journal of Fluid Mechanics, 212:593-614. 
[Li et al., 2004] Li, M., Kudo, H., Hu, J., and Johnson, R. (2004). Improved iterative algorithm for sparse object reconstruction and its performance evaluation with micro-ct data. Nuclear Science, IEEE Transactions on, 51(3):659-666.

[Lin, 2006] Lin, J. (2006). Étude détaillée des structures cohérentes de la zone tampon de la turbulence de paroi à l'aide de données de PIV stéréoscopique. $\mathrm{PhD}$ thesis, École Centrale de Lille.

[Lin et al., 2008] Lin, J., Laval, J.-P., Foucaut, J.-M., and Stanislas, M. (2008). Quantitative characterization of coherent structures in the buffer layer of near-wall turbulence. part 1: streaks. Experiments in fluids, 45(6):999-1013.

[Lu and Willmarth, 1973] Lu, S. and Willmarth, W. (1973). Measurements of the structure of the reynolds stress in a turbulent boundary layer. Journal of Fluid Mechanics, 60(03):481-511.

[Lu and Yin, 2004] Lu, W. and Yin, F.-F. (2004). Adaptive algebraic reconstruction technique. Medical physics, 31:3222.

[Lynch and Scarano, 2014] Lynch, K. and Scarano, F. (2014). Experimental determination of tomographic piv accuracy by a 12-camera system. Measurement Science and Technology, 25(8):084003.

[Lynch and Scarano, 2015] Lynch, K. and Scarano, F. (2015). An efficient and accurate approach to mte-mart for time-resolved tomographic piv. Experiments in Fluids, 56(3):1-16.

[Maas et al., 1993] Maas, H., Gruen, A., and Papantoniou, D. (1993). Particle tracking velocimetry in three-dimensional flows. Experiments in Fluids, 15(2):133-146.

[Mangasarian, 1999] Mangasarian, O. (1999). Minimum-support solutions of polyhedral concave programs. Optimization, 45(1-4):149-162.

[Martins et al., 2016] Martins, R., Pereira, A., Mompean, G., Thais, L., and Thompson, R. (2016). An objective perspective for classic flow classification criteria. Comptes Rendus Mécanique, 344(1):52-59.

[Marusic, 2001] Marusic, I. (2001). On the role of large-scale structures in wall turbulence. Physics of Fluids, 13(3):735-743.

[Marusic et al., 2010] Marusic, I., Mathis, R., and Hutchins, N. (2010). Predictive model for wall-bounded turbulent flow. Science, 329(5988):193-196. 
[Mathieu and Scott, 2000] Mathieu, J. and Scott, J. (2000). An Introduction to Turbulent Flow. Cambridge University Press.

[Adrian et al., 2000a] Adrian, R., Christensen, K., and Liu, Z.-C. (2000a). Analysis and interpretation of instantaneous turbulent velocity fields. Experiments in fluids, 29(3):275-290.

[Adrian et al., 2000b] Adrian, R., Meinhart, C., and Tomkins, C. (2000b). Vortex organization in the outer region of the turbulent boundary layer. Journal of Fluid Mechanics, 422:1-54.

[Ganapathisubramani, 2013] Ganapathisubramani, B. (2013). A tale of convection velocities. In PIV'13 - $10^{\text {th }}$ International Symposium on Particle Image Velocimetry, Delft, The Netherlands.

[Ganapathisubramani et al., 2003] Ganapathisubramani, B., Longmire, E., and Marusic, I. (2003). Characteristics of vortex packets in turbulent boundary layers. Journal of Fluid Mechanics, 478:35-46.

[Ganapathisubramani et al., 2006] Ganapathisubramani, B., Longmire, E., and Marusic, I. (2006). Experimental investigation of vortex properties in a turbulent boundary layer. Physics of Fluids, 18(5):055105.

[Minerbo, 1979] Minerbo, G. (1979). Ment: A maximum entropy algorithm for reconstructing a source from projection data. Computer Graphics and Image Processing, 10(1):48-68.

[Westerweel, 1994] Westerweel, J. (1994). Efficient detection of spurious vectors in particle image velocimetry data. Experiments in Fluids, 16(34):236-247.

[McDonough, 2007] McDonough, J. (2007). Introductory lectures on turbulence: physics, mathematics and modeling. University Lecture.

[Mejia-Alvarez and Christensen, 2013] Mejia-Alvarez, R. and Christensen, K. (2013). Robust suppression of background reflections in piv images. Measurement Science and Technology, 24(2):27003.

[Michaelis et al., 2015] Michaelis, D., Neal, D., and Wieneke, B. (2015). Peaklocking reduction for particle image velocimetry. In $11^{\text {th }}$ International Symposium on Particle Image Velocimetry - PIV15, Santa Barbara, USA.

[Michaelis et al., 2010] Michaelis, D., Novara, M., Scarano, F., and Wieneke, B. (2010). Comparison of volume reconstruction techniques at different 
particle densities. In $15^{\text {th }}$ International Symposium on Applications of Laser Techniques to Fluid Mechanics, Lisbon, Portugal.

[Mishra et al., 1999] Mishra, D., Muralidhar, K., and Munshi, P. (1999). A robust mart algorithm for tomographic applications. Numerical Heat Transfer, Part B: Fundamentals, 35(4):485-506.

[Moffat, 1988] Moffat, R. (1988). Describing the uncertainties in experimental results. Experimental thermal and fluid science, 1(1):3-17.

[Moin, 2009] Moin, P. (2009). Revisiting taylor's hypothesis. Journal of Fluid Mechanics, 640:1-4.

[Moreland, 2009] Moreland, K. (2009). Diverging color maps for scientific visualization. In Advances in Visual Computing, pages 92-103. Springer.

[Moser et al., 1999] Moser, R., Kim, J., and Mansour, N. (1999). Direct numerical simulation of turbulent channel flow up to $R e_{\tau}=590$. Physics of Fluids, 11(4):943-945.

[Nakagawa and Nezu, 1981] Nakagawa, H. and Nezu, I. (1981). Structure of space-time correlations of bursting phenomena in an open-channel flow. Journal of Fluid Mechanics, 104:1-43.

[Nogueira et al., 1999] Nogueira, J., Lecuona, A., and Rodriguez, P. (1999). Local field correction piv: on the increase of accuracy of digital piv systems. Experiments in fluids, 27(2):107-116.

[Novara, 2013] Novara, M. (2013). Advances in tomographic PIV. TU Delft, Delft University of Technology.

[Novara et al., 2010] Novara, M., Batenburg, K., and Scarano, F. (2010). Motion tracking-enhanced mart for tomographic piv. Measurement science and technology, 21(3):35401.

[Novara and Scarano, 2012] Novara, M. and Scarano, F. (2012). Performances of motion tracking enhanced tomo-piv on turbulent shear flows. Experiments in fluids, 52(4):1027-1041.

[Oldaker and Tiederman, 1977] Oldaker, D. and Tiederman, W. (1977). Spatial structure of the viscous sublayer in drag-reducing channel flows. Physics of Fluids (1958-1988), 20(10):S133-S144.

[Panton, 1997] Panton, R. (1997). Self-sustaining mechanisms of wall turbulence. Computational Mechanics, 15. 
[Pereira et al., 2000] Pereira, F., Gharib, M., Dabiri, D., and Modarress, D. (2000). Defocusing digital particle image velocimetry: a 3-component 3-dimensional dpiv measurement technique. application to bubbly flows. Experiments in Fluids, 29(1):S078-S084.

[Perry et al., 1986] Perry, A., Henbest, S., and Chong, M. (1986). A theoretical and experimental study of wall turbulence. Journal of Fluid Mechanics, 165:163-199.

[Petra et al., 2007] Petra, S., Schnörr, C., Schröder, A., and Wieneke, B. (2007). Tomographic image reconstruction in experimental fluid dynamics: Synopsis and problems. Mathematical Modelling of Environmental and Life Sciences Problems ed $R$ A Bucuresti.

[Pope, 2000] Pope, S. (2000). Turbulent flows. Cambridge university press.

[Prasad, 2000] Prasad, A. (2000). Stereoscopic particle image velocimetry. Experiments in fluids, 29(2):103-116.

[Putze and Maas, 2008] Putze, T. and Maas, H.-G. (2008). 3d determination of very dense particle velocity fields by tomographic reconstruction from four camera views and voxel space tracking. International Archives of Photogrammetry, Remote Sensing and Spatial Information Sciences, 37(B5):3338.

[Raffel et al., 2007] Raffel, M., Willert, C., and Kompenhans, J. (2007). Particle Image Velocimetry: A Practical Guide. Springer.

[Rettmann et al., 2000] Rettmann, M., Han, X., and Prince, J. (2000). Watersheds on the cortical surface for automated sulcal segmentation. In IEEE Workshop on Mathematical Methods in Biomedical Image Analysis, pages 20-27.

[Richardson, 1922] Richardson, L. (1922). Weather prediction by numerical process. Cambridge University Press.

[Robinson, 1991a] Robinson, S. (1991a). Coherent motions in the turbulent boundary layer. Annual Review of Fluid Mechanics, 23(1):601-639.

[Robinson, 1991b] Robinson, S. (1991b). The kinematics of turbulent boundary layer structure. NASA Technical Memorandum, 91:26465.

[Roerdink and Meijster, 2000] Roerdink, J. and Meijster, A. (2000). The watershed transform: Definitions, algorithms and parallelization strategies. Fundamenta Informaticae, 41(1-2):187-228. 
[Rogowitz and Treinish, 1998] Rogowitz, B. and Treinish, L. (1998). Data visualization: the end of the rainbow. Spectrum, IEEE, 35(12):52-59.

[Rohsenow and Hartnett, 1973] Rohsenow, W. and Hartnett, J. (1973). Handbook of heat transfer. McGraw-Hill Book Co., New York, NY, $5^{\text {th }}$ edition.

[Scarano, 2013] Scarano, F. (2013). Tomographic piv: principles and practice. Measurement Science and Technology, 24(1):012001.

[Scarano and Poelma, 2009] Scarano, F. and Poelma, C. (2009). Threedimensional vorticity patterns of cylinder wakes. Experiments in fluids, 47(1):69-83.

[Scarano and Riethmuller, 2000] Scarano, F. and Riethmuller, M. (2000). Advances in iterative multigrid piv image processing. Experiments in Fluids, 29(1):S051-S060.

[Schanz et al., 2016] Schanz, D., Gesemann, S., and Schröder, A. (2016). Shake-the-box: Lagrangian particle tracking at high particle image densities. Experiments in Fluids, 57(5):1-27.

[Schanz et al., 2013a] Schanz, D., Gesemann, S., Schröder, A., Wieneke, B., and Novara, M. (2013a). Non-uniform optical transfer functions in particle imaging: calibration and application to tomographic reconstruction. Measurement Science and Technology, 24(2):024009.

[Schanz et al., 2014] Schanz, D., Schröder, A., and Gesemann, S. (2014). shake the box-a 4d ptv algorithm: Accurate and ghostless reconstruction of lagrangian tracks in densely seeded flows. In 17th International Symposium on Applications of Laser Techniques to Fluid Mechanics, Lisbon, Portugal.

[Schanz et al., 2013b] Schanz, D., Schröder, A., Gesemann, S., Michaelis, D., and Wieneke, B. (2013b). shake the box: A highly efficient and accurate tomographic particle tracking velocimetry (TOMO-PTV) method using prediction of particle positions. In 10th International Symposium on PIV, Delft, The Netherlands.

[Schlichting, 1979] Schlichting, H. (1979). Boundary Layer Theory. McGrawHill, New York.

[Schlichting and Gersten, 2000] Schlichting, H. and Gersten, K. (2000). Boundary-layer theory. Springer Science \& Business Media. 
[Schnars and Jüptner, 1994] Schnars, U. and Jüptner, W. (1994). Direct recording of holograms by a ccd target and numerical reconstruction. Applied optics, 33(2):179-181.

[Schoppa and Hussain, 2000] Schoppa, W. and Hussain, F. (2000). Coherent structure dynamics in near-wall turbulence. Fluid Dynamics Research, 26(2):119-139.

[Schoppa and Hussain, 2002] Schoppa, W. and Hussain, F. (2002). Coherent structure generation in near-wall turbulence. Journal of fluid Mechanics, 453(1):57-108.

[Schröder et al., 2008a] Schröder, A., Geisler, R., Elsinga, G., Scarano, F., and Dierksheide, U. (2008a). Investigation of a turbulent spot and a tripped turbulent boundary layer flow using time-resolved tomographic piv. Experiments in Fluids, 44(2):305-316.

[Schröder et al., 2011] Schröder, A., Geisler, R., Staack, K., Elsinga, G.,

Scarano, F., Wieneke, B., Henning, A., Poelma, C., and Westerweel, J. (2011). Eulerian and lagrangian views of a turbulent boundary layer flow using time-resolved tomographic piv. Experiments in fluids, 50(4):1071-1091.

[Schröder et al., 2008b] Schröder, A., Geisler, R., Staack, K., Wieneke, B., Elsinga, G., Scarano, F., and Henning, A. (2008b). Lagrangian and eulerian views into a turbulent boundary layer flow using time-resolved tomographic piv. In 14th International Symposium on Applications of Laser Techniques to Fluid Mechanics, Lisbon, Portugal.

[Schröder et al., 2015] Schröder, A., Schanz, D., Michaelis, D., Cierpka, C., Scharnowski, S., and Kähler, C. (2015). Advances of piv and 4d-ptv shakethe-box for turbulent flow analysis-the flow over periodic hills. Flow, Turbulence and Combustion, 95(2-3):193-209.

[Sciacchitano et al., 2014] Sciacchitano, A., Neal, D., Smith, B., Warner, S., Vlachos, P., Wieneke, B., and Scarano, F. (2014). Collaborative framework for piv uncertainty quantification: comparative assessment of methods. In 17th International Symposium on Applications of Laser Techniques to Fluid Mechanics, Lisbon, Portugal.

[Sheng et al., 2008] Sheng, J., Malkiel, E., and Katz, J. (2008). Using digital holographic microscopy for simultaneous measurements of $3 \mathrm{~d}$ near wall velocity and wall shear stress in a turbulent boundary layer. Experiments in fluids, 45(6):1023-1035. 
[Smith and Metzler, 1983] Smith, C. and Metzler, S. (1983). The characteristics of low-speed streaks in the near-wall region of a turbulent boundary layer. Journal of Fluid Mechanics, 129:27-54.

[Smith and Walker, 1995] Smith, C. and Walker, J. (1995). Turbulent walllayer vortices. In Fluid vortices, pages 235-289. Springer.

[Smith and Walker, 1997] Smith, C. and Walker, J. (1997). Sustaining mechanisms of turbulent boundary layers: the role of vortex development and interactions. Self-sustaining Mechanism of Wall Turbulence. Boston: Computational Mechanics Publications, 47.

[Smith et al., 1991] Smith, C., Walker, J., Haidari, A., and Sobrun, U. (1991). On the dynamics of near-wall turbulence. Philosophical Transactions of the Royal Society of London. Series A: Physical and Engineering Sciences, 336(1641):131-175.

[Soloff et al., 1997] Soloff, S., Adrian, R., and Liu, Z.-C. (1997). Distortion compensation for generalized stereoscopic particle image velocimetry. Measurement science and technology, 8(12):1441.

[Stanislas et al., 2008] Stanislas, M., Perret, L., and Foucaut, J.-M. (2008). Vortical structures in the turbulent boundary layer: a possible route to a universal representation. Journal of Fluid Mechanics, 602:327-382.

[Talmon et al., 1986] Talmon, A., Kunen, J., and Ooms, G. (1986). Simultaneous flow visualization and reynolds-stress measurement in a turbulent boundary layer. Journal of Fluid Mechanics, 163:459-478.

[Tanahashi et al., 2004] Tanahashi, M., Kang, S.-J., Miyamoto, T., Shiokawa, S., and Miyauchi, T. (2004). Scaling law of fine scale eddies in turbulent channel flows up to $R e_{\tau}=800$. International journal of heat and fluid flow, 25(3):331-340.

[Theodorsen, 1952] Theodorsen, T. (1952). Mechanism of turbulence. In $2^{\text {nd }}$ Midwestern Conference on Fluid Mechanics, Ohio State University, Columbus, Ohio.

[Thomas et al., 2014] Thomas, L., Tremblais, B., and David, L. (2014). Optimisation of the volume reconstruction for classical tomo-piv algorithms (mart, bimart and smart): synthetic and experimental studies. Measurement Science and Technology, 25(3):035303. 
[Thomas et al., 2010] Thomas, L., Vernet, R., Tremblais, B., and David, L. (2010). Influence of geometric parameters and image preprocessing on tomo-piv results. In $15^{\text {th }}$ International Symposium on Application of Laser Techniques to Fluid Mechanics, Lisbon, Portugal.

[Tomkins and Adrian, 2003] Tomkins, C. and Adrian, R. (2003). Spanwise structure and scale growth in turbulent boundary layers. Journal of Fluid Mechanics, 490:37-74.

[Townsend, 1980] Townsend, A. (1980). The structure of turbulent shear flow. Cambridge University Press.

[Tsai, 1986] Tsai, R. (1986). An efficient and accurate camera calibration technique for $3 \mathrm{~d}$ machine vision. In Proceedings of the IEEE Computer Society Conference on Computer Vision and Pattern Recognition.

[Van Doorne and Westerweel, 2007] Van Doorne, C. and Westerweel, J. (2007). Measurement of laminar, transitional and turbulent pipe flow using stereoscopic-piv. Experiments in Fluids, 42(2):259-279.

[Vinuesa et al., 2014] Vinuesa, R., Noorani, A., Lozano-Durán, A., Khoury, G., Schlatter, P., Fischer, P., and Nagib, H. (2014). Aspect ratio effects in turbulent duct flows studied through direct numerical simulation. Journal of Turbulence, 15(10):677-706.

[Vollmers, 2001] Vollmers, H. (2001). Detection of vortices and quantitative evaluation of their main parameters from experimental velocity data. Measurement Science and Technology, 12(8):1199.

[Von Kármán, 1938] Von Kármán, T. (1938). Some remarks on the statistical theory of turbulence. In Proc. $5^{\text {th }}$ International Congress for Applied Mechanics, volume 347 .

[Wallace et al., 1972] Wallace, J., Eckelmann, H., and Brodkey, R. (1972). The wall region in turbulent shear flow. Journal of Fluid Mechanics, 54(01):3948.

[Wang et al., 2000] Wang, J.-J., Lan, S.-1., and Chen, G. (2000). Experimental study on the turbulent boundary layer flow over riblets surface. Fluid dynamics research, 27(4):217-229.

[Wei et al., 2005] Wei, T., Schmidt, R., and McMurtry, P. (2005). Comment on the clauser chart method for determining the friction velocity. Experiments in fluids, 38(5):695-699. 
[Westerweel et al., 2013] Westerweel, J., Elsinga, G. E., and Adrian, R. (2013). Particle image velocimetry for complex and turbulent flows. Annual Review of Fluid Mechanics, 45:409-436.

[Westerweel and Scarano, 2005] Westerweel, J. and Scarano, F. (2005). Universal outlier detection for piv data. Experiments in Fluids, 39(6):1096-1100.

[White and Mungal, 2008] White, C. M. and Mungal, M. G. (2008). Mechanics and prediction of turbulent drag reduction with polymer additives. Annual Review of Fluid Mechanics, 40:235-256.

[Wieneke, 2008] Wieneke, B. (2008). Volume self-calibration for 3d particle image velocimetry. Experiments in Fluids, 45(4):549-556.

[Wieneke, 2013] Wieneke, B. (2013). Iterative reconstruction of volumetric particle distribution. Measurement Science and Technology, 24(2):024008.

[Wieneke and Sciacchitano, 2015] Wieneke, B. and Sciacchitano, A. (2015). Piv uncertainty propagation. In $11^{\text {th }}$ International Symposium on Particle Image Velocimetry - PIV15, Santa Barbara, USA.

[Willert, 1997] Willert, C. (1997). Stereoscopic digital particle image velocimetry for application in wind tunnel flows. Measurement science and technology, 8(12):1465-1479.

[Worth and Nickels, 2008] Worth, N. and Nickels, T. (2008). Acceleration of tomo-piv by estimating the initial volume intensity distribution. Experiments in fluids, 45(5):847-856.

[Wu and Christensen, 2006] Wu, Y. and Christensen, K. T. (2006). Population trends of spanwise vortices in wall turbulence. Journal of Fluid Mechanics, 568:55-76.

[Zhou et al., 1999] Zhou, J., Adrian, R., Balachandar, S., and Kendall, T. (1999). Mechanisms for generating coherent packets of hairpin vortices in channel flow. Journal of Fluid Mechanics, 387:353-396. 